\title{
A Small-Scale Standalone Wind Energy \\ Conversion System Featuring SCIG, CSI and a Novel Storage Integration Scheme
}

\author{
by \\ Zuher Alnasir

\begin{abstract}
A thesis
presented to the University of Waterloo

in fulfillment of the

thesis requirement for the degree of

Doctor of Philosophy

in
\end{abstract}

Electrical and Computer Engineering

Waterloo, Ontario, Canada, 2016

(C) Zuher Alnasir 2016 


\section{Author's Declaration}

I hereby declare that I am the sole author of this thesis. This is a true copy of the thesis, including any required final revisions, as accepted by my examiners.

I understand that my thesis may be made electronically available to the public. 


\begin{abstract}
Small-scale standalone wind turbines provide a very attractive renewable energy source for off-grid remote communities. Taking advantage of variable-speed turbine technology, which requires a partial- or full-scale power converter, and through integrating an energy storage system, smooth and fast power flow control, maximum power point tracking, and a highquality power is ensured.

Due to high reliability and efficiency, permanent magnet synchronous generator seems to be the dominating generator type in gearless wind turbines, employed for off-grid applications. However, wind turbines using geared squirrel-cage induction generator (SCIG) are still widely accepted due to their robustness, simplicity, light weight and low cost. Permanent magnet induction generator, a relatively new induction-based machine, has recently been recognized in the wind energy market as an alternative for permanent magnet synchronous generator. A thorough comparative study, among these three generator types, is conducted in this research in order to enable selection of the most appropriate generator for off-grid wind energy conversion system (WECS), subject to a set of given conditions. The system based on geared SCIG has been shown to be the most appropriate scheme for a small-scale standalone WECS, supplying a remote area.
\end{abstract}

Different topologies of power electronic converters, employed in WECSs, are overviewed. Among the converters considered, current source converter is identified to have a great potential for off-grid wind turbines.

Three current-source inverter-based topologies, validated in the literature for on-grid WECS, are compared for off-grid WECS application. Feasibility study and performance evaluation are conducted through analysis and simulation. Among all, the topology composed of three-phase diode bridge rectifier, DC/DC buck converter, and pulse-width-modulated current-source inverter (PWM-CSI) is identified as a simple and low-cost configuration, offering satisfactory performance for a low-power off-grid WECS.

A small-scale standalone wind energy conversion system featuring SCIG, CSI and a novel energy storage integration scheme is proposed and a systematic approach for the dc-link inductor design is presented. 
In developing the overall dynamic model of the proposed wind turbine system, detailed models of the system components are derived. A reduced-order generic load model, that is suitable for both balanced and unbalanced load conditions, is developed and combined with the system components in order to enable steady-state and transient simulations of the overall system. A linear small-signal model of the system is developed around three operating points to investigate stability, controllability, and observability of the system. The eigenvalue analysis of the small-signal model shows that the open-loop system is locally stable around operating points 1 and 3, but not 2. Gramian matrices of the linearized system show that the system is completely controllable at the three operating points and completely observable at operating points 1 and 3 , but not 2 .

The closed-loop control system for the proposed wind turbine system is developed. An effective power management algorithm is employed to maintain the supply-demand power balance through direct control of dc-link current. The generator's shaft speed is controlled by the buck converter to extract maximum available wind power in normal mode of operation. The excess wind power is dumped when it is not possible to absorb maximum available power by the storage system and the load. The current source inverter is used to control positive- and negative-sequence voltage components separately. The feasibility of the proposed WECS and performance of the control system under variable wind and balanced/unbalanced load conditions are analyzed and demonstrated through simulation.

Finally, the proposed WECS is modified by removing the dump load and avoiding the surplus power generation by curtailment of wind power. The operation of the modified system is investigated and verified under variable wind and load conditions. 


\section{Acknowledgements}

First and above all, I praise the almighty ALLAH for granting me the capability to proceed successfully. This thesis would not have been possible without the support of several individuals who in one way or another contributed their valuable assistance in the completion of this work.

I would like to express my utmost gratitude and thanks to my academic supervisor, Prof. Mehrdad Kazerani, who has advised, guided and supported me throughout this research work.

Sincere thanks are also given to my $\mathrm{PhD}$ committee members: Prof. Claudio Canizares and Prof. Magdy Salama from the department of Electrical and Computer Engineering at University of Waterloo, and Prof. Amir Khajepour from the department of Mechanical and Mechatronics Engineering at University of Waterloo, for their valuable feedback and suggestions during my $\mathrm{PhD}$ comprehensive examination and seminar. It is my pleasure to thank Prof. Alireza Bakhshai from the department of Electrical and Computer Engineering at Queen's University, for being my external examiner.

Ethically, I should not forget to thank my parents, my wife, and my sisters and brothers for their moral support during the period of my PhD program.

Finally, I would like to acknowledge the financial support received from the Royal Commission for Jubail \& Yanbu (RCJY) in the Kingdom of Saudi Arabia through the Saudi culture bureau in CANADA. 


\section{Dedication}

To my Lovely Daughter,

“ASEEL”,

The sunshine of my life 


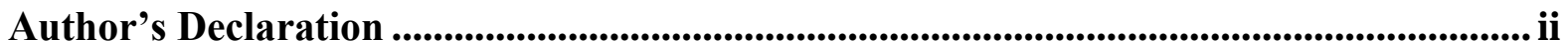

Abstract.......................................................................................iii

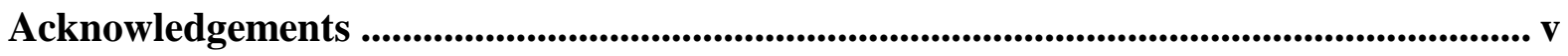

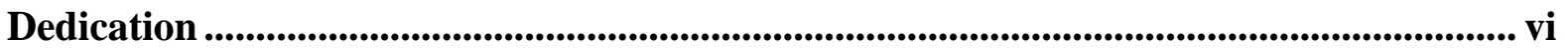

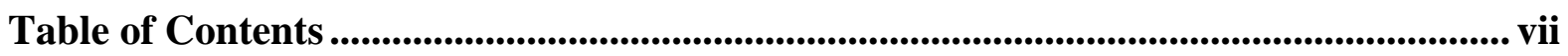

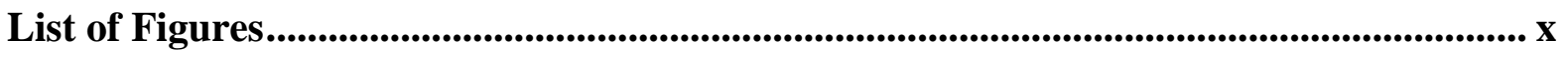

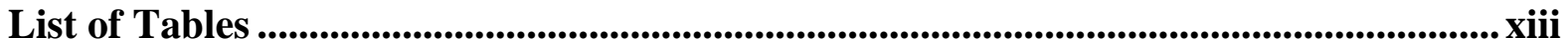

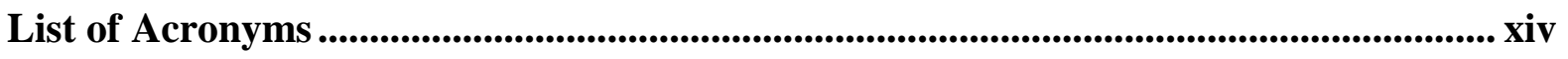

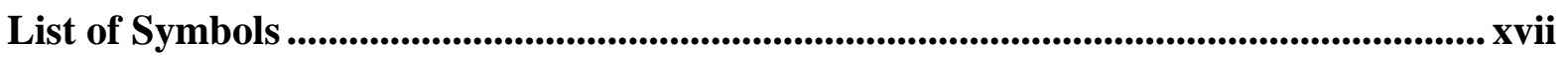

Chapter 1 Introduction ............................................................................................................................. 1

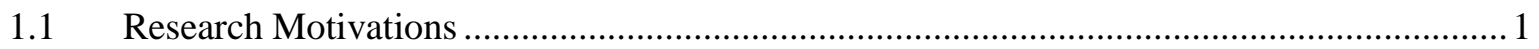

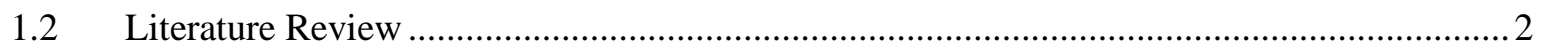

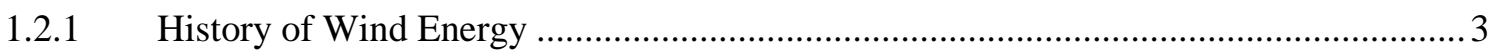

1.2.2 Conventional and Potential Generator Types used in WECS......................................... 3

1.2.3 Power Electronic Converter Topologies for Standalone WECS ................................ 9

1.2.4 PWM-CSI versus PWM-VSI for Standalone WECS ............................................... 13

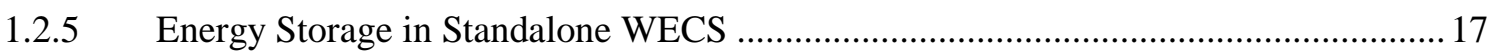

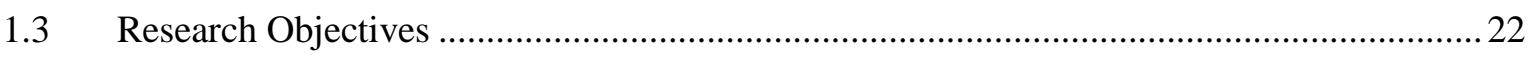

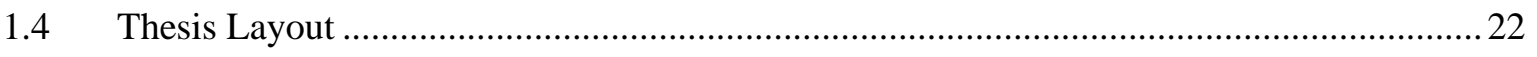

Chapter 2 Selection of Generator Type for Small-Scale WECS ...................................... 24

2.1 Evaluation of Conventional and Evolving Generator Types for Standalone WECS ...........24

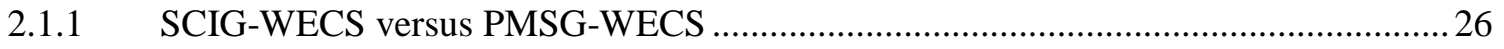

2.1.2 PMIG versus SCIG and PMSG for Standalone WECS .............................................. 33

2.1.3 Indices for Selecting the Preferred Generator ............................................................. 35

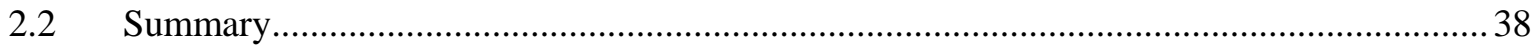

Chapter 3 Proposed Wind Energy Conversion System ....................................................... 39

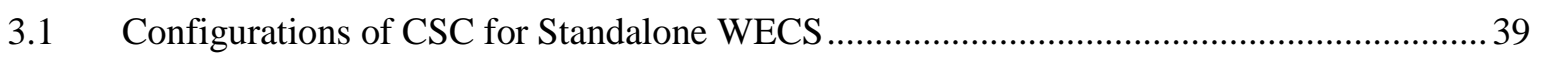

3.1.1 Topology 1: Diode Rectifier - PWM CSI................................................................ 40

3.1.2 Topology 2: Diode Rectifier - Buck Converter - PWM CSI ....................................... 42

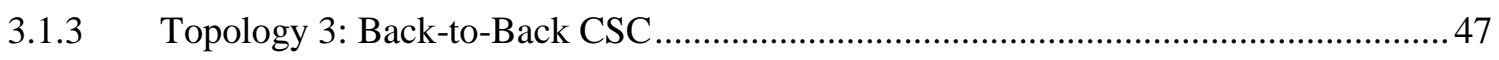


3.2 Comparison of Different CSC-WECS Topologies......................................................50

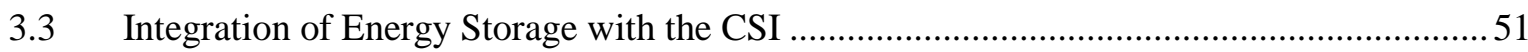

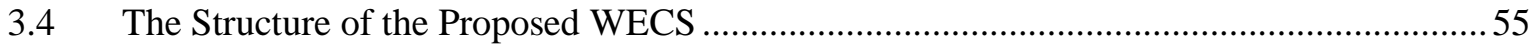

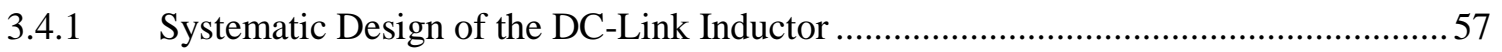

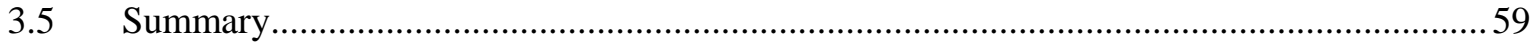

Chapter 4 Dynamic Modeling and Small Signal Analysis of the CSI-WECS ................. 61

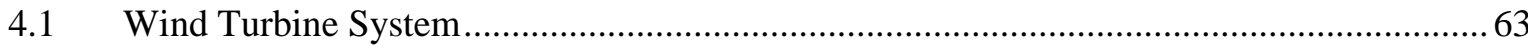

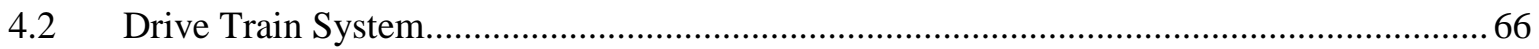

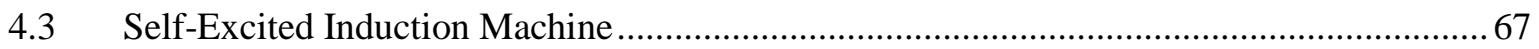

4.4 Three-Phase Diode Bridge with DC-Side Capacitive Filter .............................................. 71

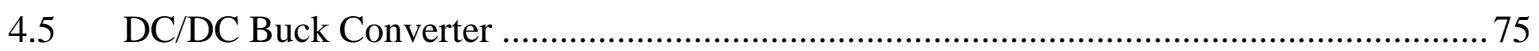

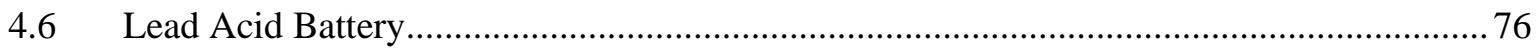

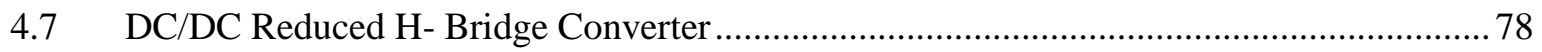

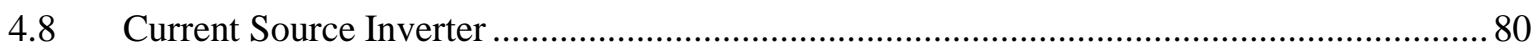

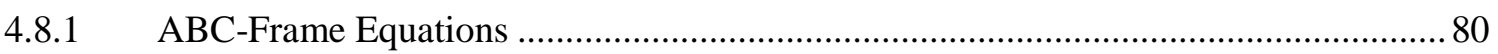

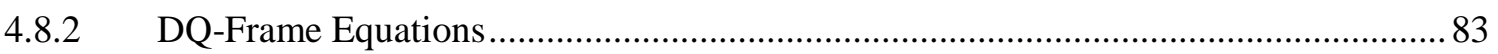

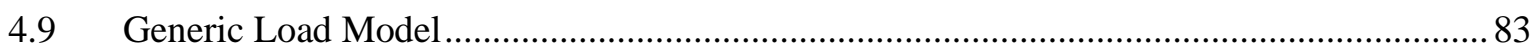

4.9.1 Generic Load Model Proposed in [138] …............................................................ 84

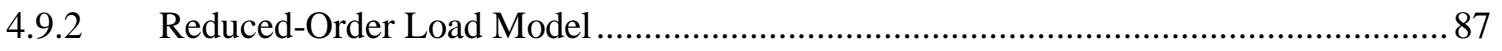

4.9.3 Generic Load Model including Unbalanced Load Condition....................................... 88

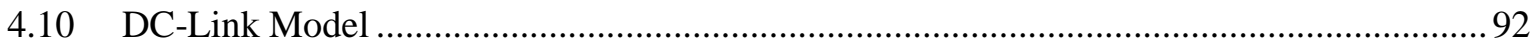

4.11 Dynamic Model of the Proposed Wind Energy Conversion System .................................93

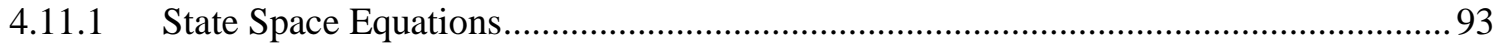

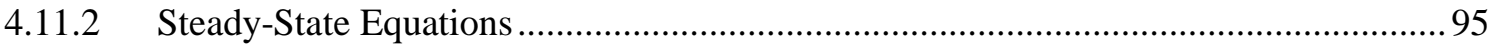

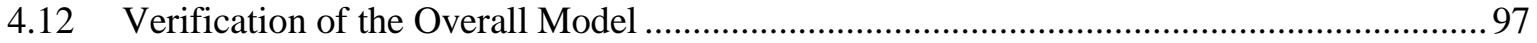

4.12.1 Wind Turbine Generation (WTG) Subsystem ..........................................................98

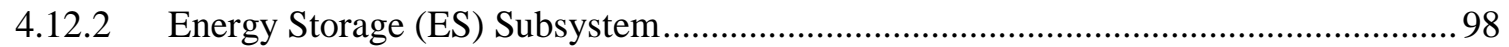

4.12.3 Current-Sourced Inverter-Load (CSI-Load) Subsystem ............................................99

4.12.4 Starting Operation of the Wind Turbine System ................................................... 100

4.12.5 Average model versus Switching model for Power Electronics Converters .............. 101

4.12.6 Open-Loop Step Responses of the System .............................................................. 104

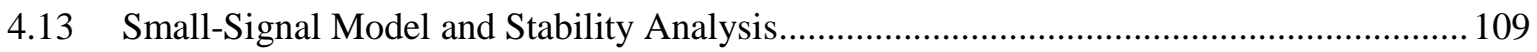

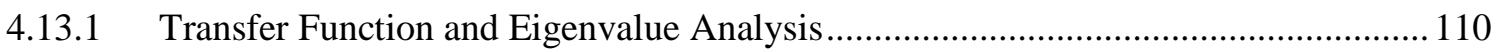

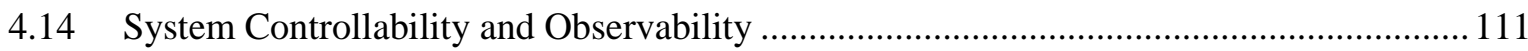




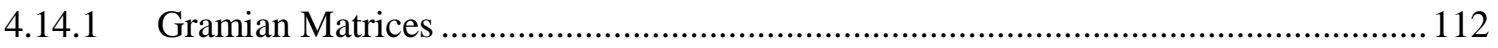

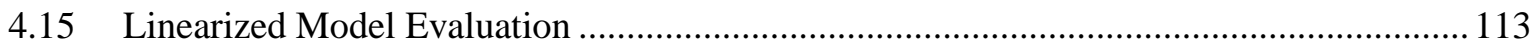

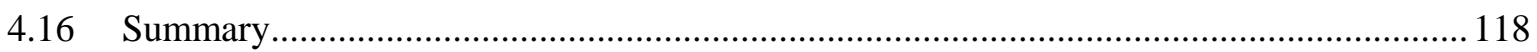

\section{Chapter 5 Control System Design for the Proposed PWM-CSI-Based SCIG-WECS. 119}

5.1 Overview of the control system of the proposed WECS …............................................. 119

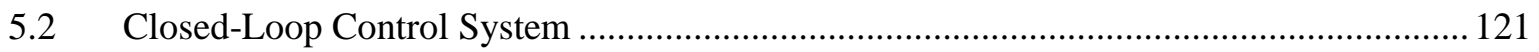

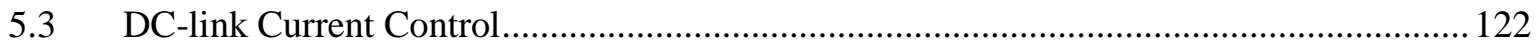

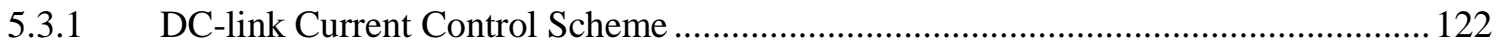

5.3.2 Parameters Design of DC-link Current PI Controller................................................... 126

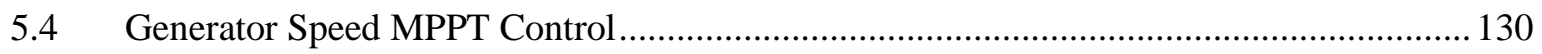

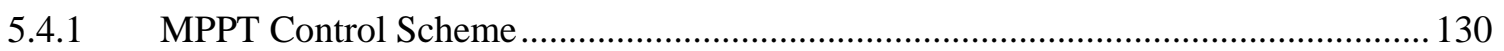

5.4.2 Parameter Design of Generator Speed Control Loop ............................................... 132

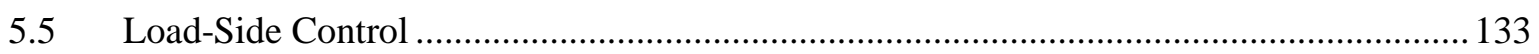

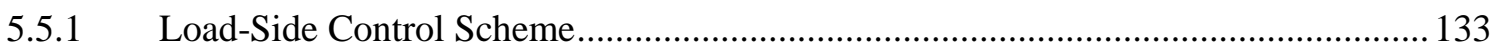

5.5.2 Parameter Design of Load-Side Control Loop ........................................................ 134

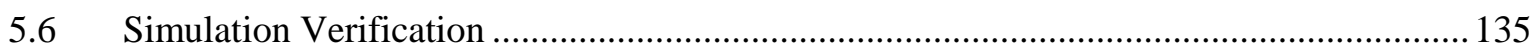

5.6.1 System Performance under Various Wind Speed and Load Conditions .................... 136

5.6.2 Effect of System Inertia and Frequency of Wind Speed Variation on MPPT ............ 143

5.6.3 Performance of the Synchronous $d q$ Controller under Various Dynamics of Load... 144

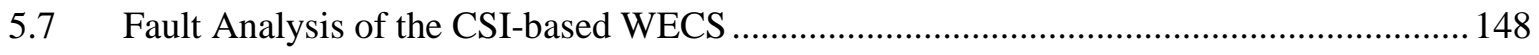

5.8 Dump Load-Less Version of the Proposed CSI-based WECS ….................................... 152

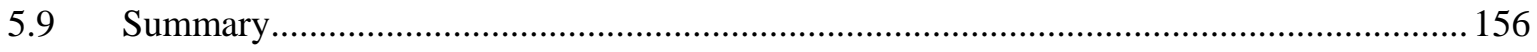

Chapter 6 Conclusions, Contributions, and Future Work .......................................... 158

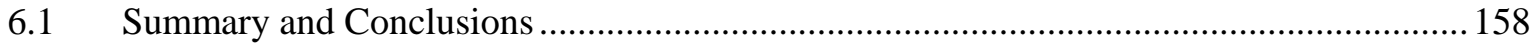

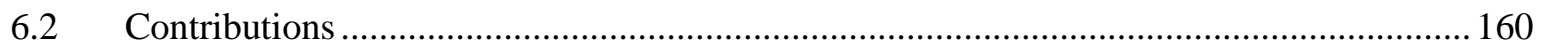

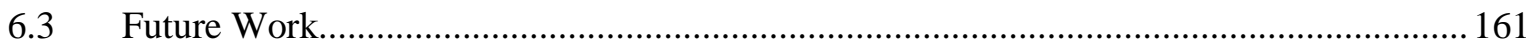

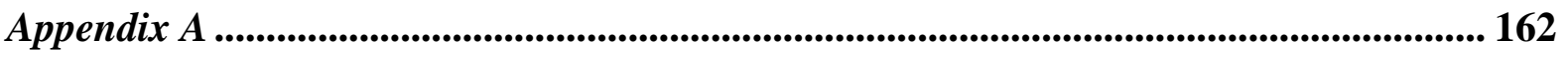

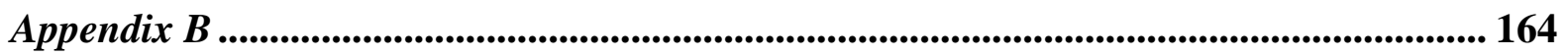

Appendix $C$................................................................................................................................ 165

Appendix D.................................................................................................................................. 167

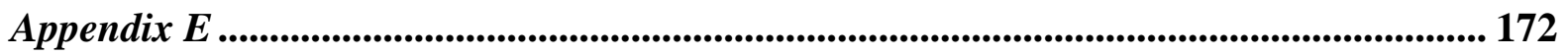

References ........................................................................................................................................ 182 


\section{List of Figures}

Fig. 1.1: Global annual installed Small Wind turbine (SWT)[2]. ....................................................

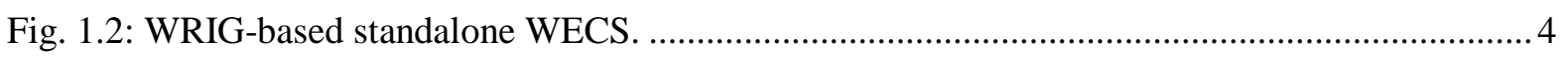

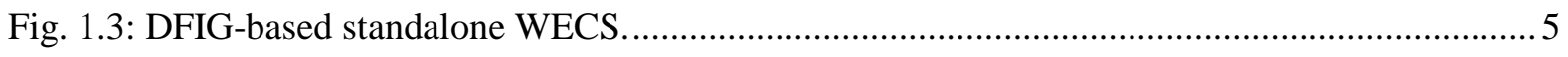

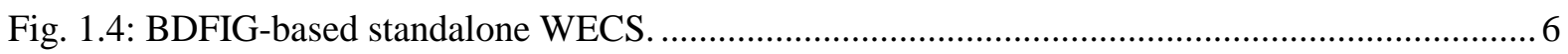

Fig. 1.5: WRSG-based direct-drive standalone WECS................................................................. 7

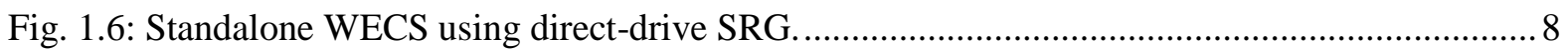

Fig. 1.7: Diode rectifier + dc/dc boost converter + VSI. ................................................................ 9

Fig. 1.8: Two-level, back-to-back voltage-source converter topology .............................................. 10

Fig. 1.9: Back-to-back current-source converter topology ............................................................ 11

Fig. 1.10: Diode Rectifier Bridge + Z-Source inverter................................................................... 11

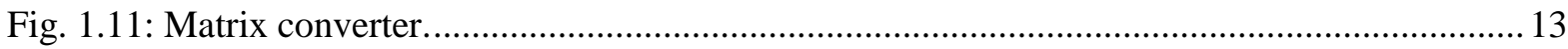

Fig. 2.1: SCIG-based standalone WECS: (a) with generator-side diode bridge rectifier, and (b) with generator-side voltage-source rectifier.

Fig. 2.2: PMSG-based direct-drive standalone WECS: (a) with generator-side diode bridge rectifier, and (b) with generator-side voltage-source rectifier. .28

Fig. 2.3: PMIG-based direct-drive standalone WECS: (a) with generator-side diode bridge rectifier,

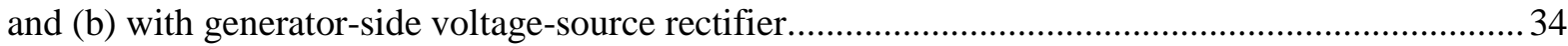

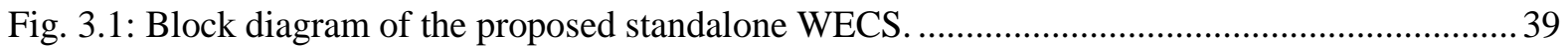

Fig. 3.2:A SCIG-WECS composed of a diode rectifier and a PWM-CSI......................................... 41

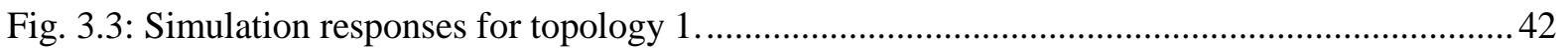

Fig. 3.4: A SCIG-WECS composed of a diode rectifier, a buck converter and a PWM-CSI. ............. 43

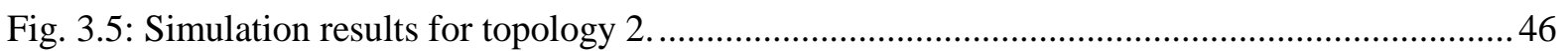

Fig. 3.6: Effect of diode bridge rectifier on generator characteristics at rated speed. ......................... 46

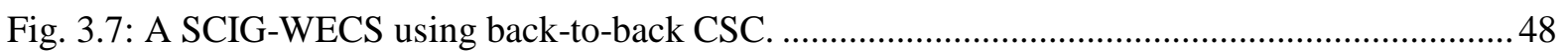

Fig. 3.8: MPPT for back-to-back CSC-based WECS........................................................................ 49

Fig. 3.9: Stator current and generator electromagnetic torque at rated speed. .................................. 49

Fig. 3.10: Integration of the energy storage system proposed in [70] with the CSI- based WECS of

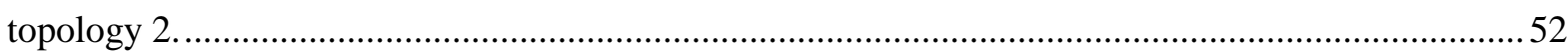

Fig. 3.11: Simulation results for the CSI-based WECS with energy storage system proposed in [70].

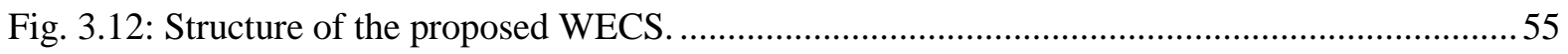

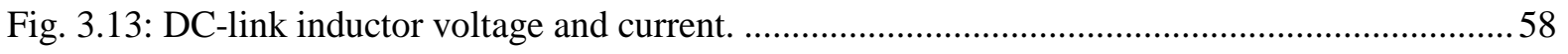

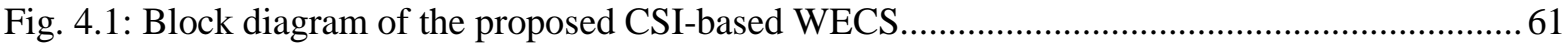


Fig. 4.2: Performance coefficient versus tip speed ratio for various blade pitch angles. .64

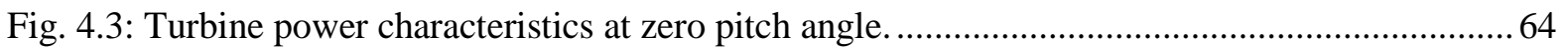

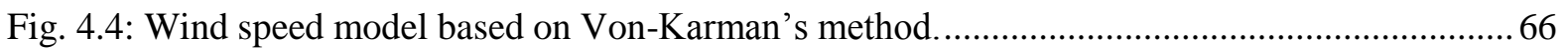

Fig. 4.5: Two mass model of turbine's drive train on Turbine side. ................................................. 67

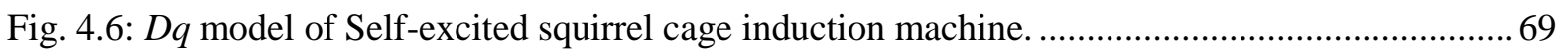

Fig. 4.7: Three-phase diode bridge rectifier with a variable dc current. ........................................... 71

Fig. 4.8: Three-phase diode bridge rectifier with a constant dc current........................................... 73

Fig. 4.9: Averaged model of CCM diode bridge rectifier with instantaneous commutation................74

Fig. 4.10: $D q$ average model of diode bridge rectifier supplied by SEIG. ......................................... 74

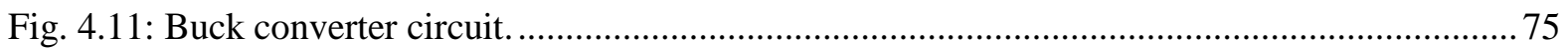

Fig. 4.12: Large-signal nonlinear averaged model of CCM buck converter...................................... 76

Fig. 4.13: Electrical model of a battery cell. ................................................................................. 76

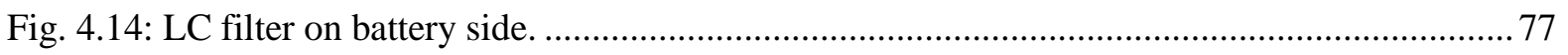

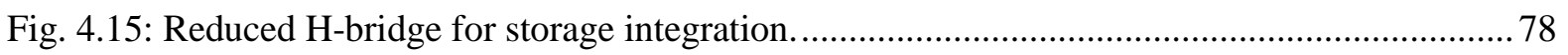

Fig. 4.16: Large-signal nonlinear averaged model of CCM Full-Bridge Converter. ........................... 79

Fig. 4.17: Three-phase current-sourced inverter feeding a three-phase load...................................... 80

Fig. 4.18: Large-signal nonlinear averaged model of current source inverter.................................... 82

Fig. 4.19: Block diagram of the generic load model proposed in [138] ........................................... 85

Fig. 4.20: Reduced-order model versus the original 4th -order model.............................................. 87

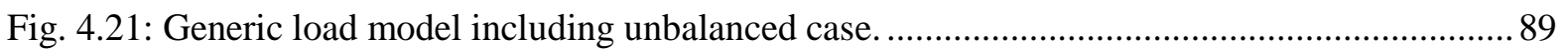

Fig. 4.22: Load currents and powers using the extended generic load model....................................92

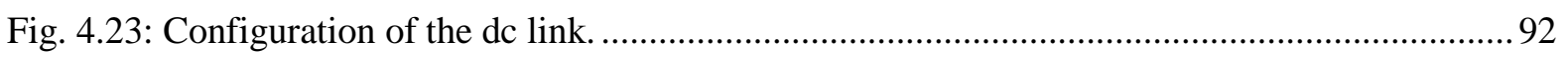

Fig. 4.24: Inputs, state variables, and outputs of the proposed WECS .............................................. 95

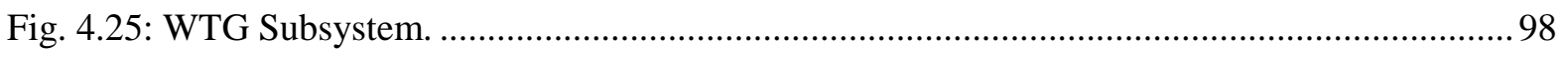

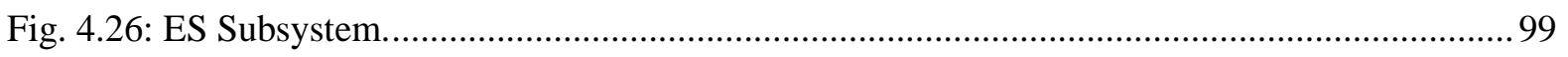

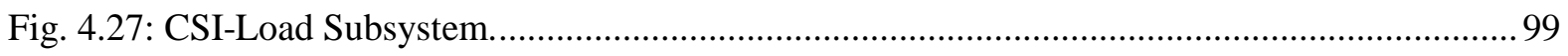

Fig. 4.28: Starting of the wind turbine under unloaded/loaded conditions. ..................................... 101

Fig. 4.29: Average model versus switching model: diode bridge rectifier waveforms. .................... 102

Fig. 4.30: Switching and average model waveforms of buck converter........................................... 103

Fig. 4.31: Switching and average model waveforms of recued-H-bridge converter......................... 103

Fig. 4.32: Switching and average model waveforms of CSI. ......................................................... 104

Fig. 4.33: Responses of generator rotor speed and active power to 50\% step decrease in $d_{b} \ldots \ldots . . .105$

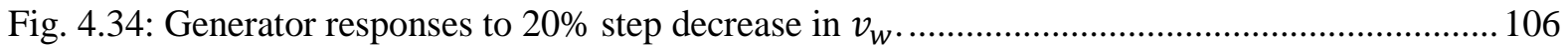

Fig. 4.35: Responses of dc-link current to $80 \%$ step change in $d_{A}$............................................... 107 


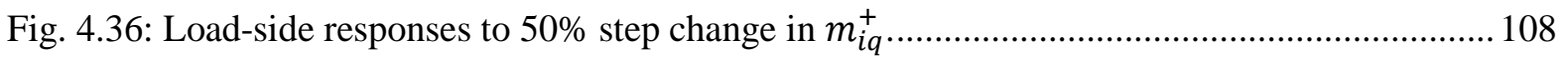

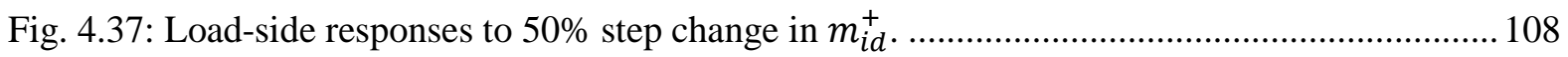

Fig. 4.38: Average and switching model load-side voltage responses to $50 \%$ step increase in $m_{i d}^{+} .109$

Fig. 5.1: Converters and control blocks in the CSI-based WECS.................................................. 120

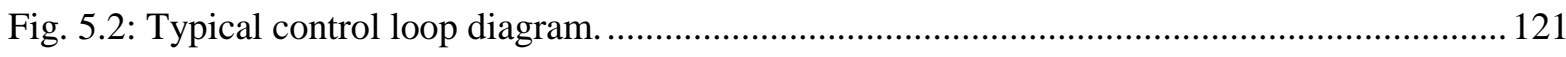

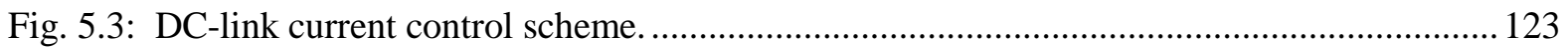

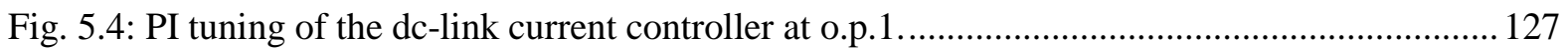

Fig. 5.5: PI tuning of the dc-link current controller at o.p.2 ........................................................ 128

Fig. 5.6: PI tuning of the dc-link current controller at o.p.3 ...................................................... 128

Fig. 5.7: Step/Ramp response of dc-link current at the three operating points................................. 129

Fig. 5.8: Typical turbine power versus wind speed curve. ............................................................ 131

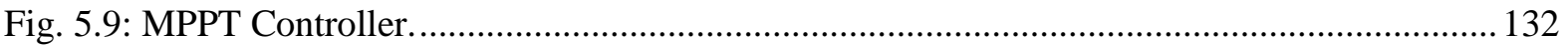

Fig. 5.10: Load-side synchronous $d q$ frame control scheme.......................................................... 134

Fig. 5.11: System behavior under variable balanced load. ............................................................ 139

Fig. 5.12: Effect of generator inductance on stator current and electromagnetic torque................... 140

Fig. 5.13: Power management after battery SoC reaches upper limit. ............................................. 141

Fig. 5.14: System behavior under variable unbalanced load.......................................................... 142

Fig. 5.15: Effects of system inertia and frequency of wind speed variation on MPPT. .................... 144

Fig. 5.16: Load characteristics under synchronous $d q$-frame controllers....................................... 146

Fig. 5.17: Load-side three-phase voltages and currents under synchronous $d q$-frame controllers..... 146

Fig. 5.18: Load-side stationary abc frame control scheme using PI controllers................................ 147

Fig. 5.19: Performance comparsion of abc-frame and $d q$-frame PI controllers. ............................... 148

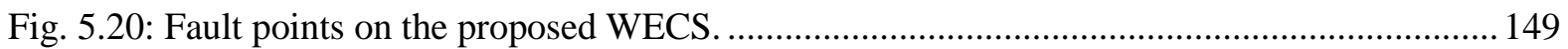

Fig. 5.21:System performance during (a) SLG (b) LL (c) TPG faults at secondary side of the $\Delta / Y n$

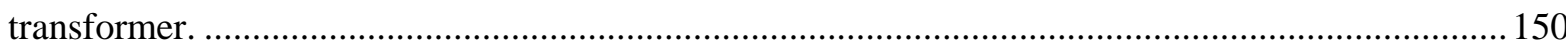

Fig. 5.22: Harmonic Spectrum of CSI's ac terminal and filtered currents before and during SLG fault.

Fig. 5.23: Inverter dc-side current of VSI-and CSI-based WECS during an SLG fault................... 152

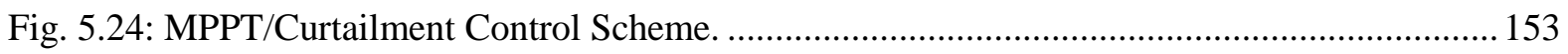

Fig. 5.25: System behavior under MPPT/Curtailment control...................................................... 155 


\section{List of Tables}

Table 1.1: Cost Comparison of 30kW PMSG-based Wind Turbines with VSI and CSI [93]............. 15

Table 1.2: Advantages and drawbacks of different energy storage technologies.............................. 18

Table 1.3: Energy storage requirements for a small-scale standalone WECS...................................20

Table 1.4: Feasibility of Energy Storage Technologies for small-scale standalone WECS................21

Table 2.1: Cost Comparison of 30kW PMSG- and SCIG-Based WECSs. ......................................... 32

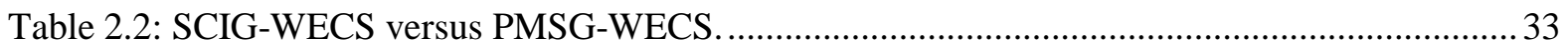

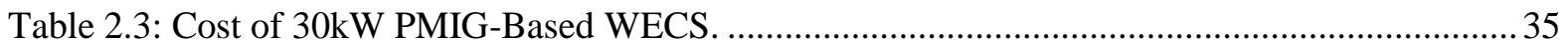

Table 2.4: Comparison of the geared-drive SCIG, gearless-drive PMSG and gearless-drive

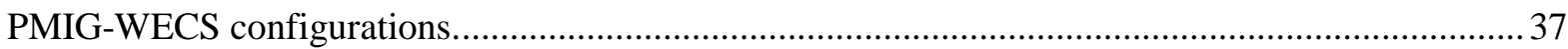

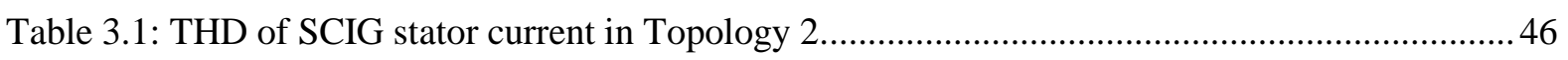

Table 3.2: Comparison of the three standalone CSC-based WECS topologies....................................51

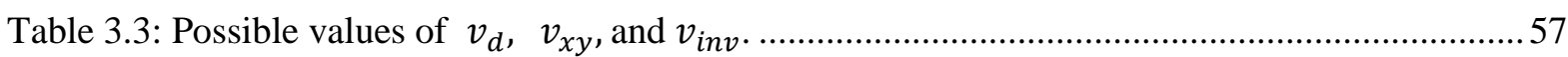

Table 4.1: Reduced H-bridge converter operating modes............................................................. 78

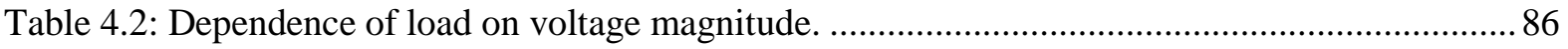

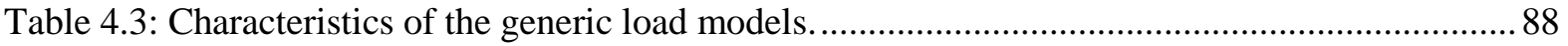

Table 4.4: Characteristics of extended generic load model............................................................ 91

Table 4.5: Input parameters for WTG subsystem used for starting simulation................................ 100

Table 4.6: Input, output and parameter values at the operating point for steady-state analysis......... 102

Table 4.7: Input, state and output variables at the operating points for linearization......................... 114

Table 4.8: Eigenvalues and damping ratios of the linearized system at o.p.1 and o.p.2 .................. 115

Table 4.9: Singular values of Gramian matrices at o.p.1 and o.p.2 ............................................... 115

Table 4.10: Eigenvalues and damping ratios of the linearized system at o.p.3 ............................... 116

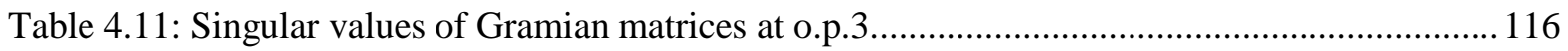

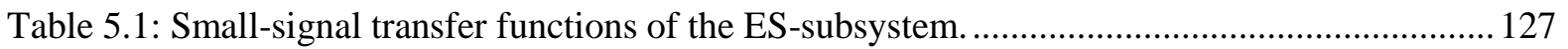

Table 5.2: PI gains of the closed loop system controllers at the three operating points.................... 135

Table 5.3: Wind speed and load conditions for simulation results of Fig. 5.11 and Fig. 5.14.......... 136

Table 5.4: Load parameters for simulation results of Fig. 5.16 .......................................................... 145

Table 5.5: Wind speed and load conditions for simulation results of Fig. 5.25. .............................. 155

Table A.1: Parameters and operating condition of the 30kW-CSC-SCIG WECS....................162

Table A.2: Parameters and rated operating conditions of the 20kW-CSI-SCIG-WECS.................163

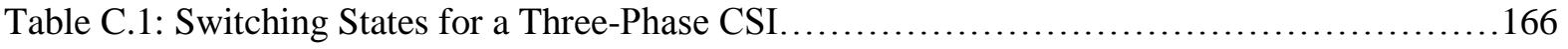




\section{List of Acronyms}

\begin{tabular}{ll} 
+ve seq & Positive Sequence \\
2L-BTB-VSC & Two-Level Back-to-Back Voltage-Source Converter \\
BDFIG & Brushless Doubly-Fed Induction Generator \\
BDFRG & Brushless Doubly-Fed Reluctance Generator \\
BTB-CSC & Back-to-Back Current-Source Converter \\
CAES & Compressed Air Energy Storage \\
CCM & Continuous Conduction Mode \\
CL & Closed Loop \\
CLTF & Closed-Loop Transfer Function \\
CSC & Current-Source Converter \\
CSI-Load & Current Source Inverter-Load \\
DCM & Discontinues Conduction Mode \\
DFIG & Doubly-Fed Induction Generator \\
DOD & Depth of Discharge \\
DRFOC & Direct Rotor Flux Oriented Control \\
ES & Energy Storage \\
ESR & Equivalent Series Resistance \\
FBES & Flow Battery Energy Storage \\
FES & Flywheel Energy Storage \\
HES & Hydrogen Energy Storage \\
IG & Induction Generator \\
IGBT & Insulated-Gate Bipolar Transistor \\
IMC & Indirect Matrix Converter \\
LAB & Lead Acid Battery \\
LIES & Lithium Ion battery Energy Storage \\
LUF & Load Unbalance Factor \\
MC & Matrix Converter \\
ML & Multi-Level \\
MPPT & Maximum Power Point Tracking \\
NaSES & Sodphur battery Energy Storage \\
\hline
\end{tabular}


NCES

O\&M

o.p.

OL

OLTF

OSF

PCC

PHES

PM

PMIG

PMSG

PWM

PWM-CSI

PWM-CSR

PWM-VSI

RMS

SCIG

SEIG

Seq

SES

SMES

$\mathrm{SoC}$

SRG

SSF

SVD

SWT

THD

-ve seq

VSC

VSI

VSR
Nickel Cadmium battery Energy Storage

Operation and Maintenance

Operating point

Open Loop

Open-Loop Transfer Function

Open-Switch Fault

Point of Common Coupling

Pumped Hydro Energy Storage

Permanent Magnet

Permanent-Magnet Induction Generator

Permanent-Magnet Synchronous Generator

Pulse Width Modulation

Pulse-Width Modulated Current-Source Inverter

Pulse-Width Modulated Current-Source Rectifier

Pulse-Width Modulated Voltage-Source Inverter

Root Mean Square

Squirrel-Cage Induction Generator

Self-Excited Induction Generator

Sequence

Super-capacitor Energy storage

Superconducting Magnetic Energy Storage

State of Charge

Switched-Reluctance Generator

Short-Switch Fault

Singular Value Decomposition

Small Wind turbine

Total Harmonic Distortion

Negative Sequence

Voltage-Source Converter

Voltage-Source Inverter

Voltage-Source Rectifier 


$\begin{array}{ll}\text { WECS } & \text { Wind Energy Conversion system } \\ \text { WRIG } & \text { Wound-Rotor Induction Generator } \\ \text { WRSG } & \text { Wound-Rotor Synchronous Generator } \\ \text { WT } & \text { Wind turbine } \\ \text { WTG } & \text { Wind-Turbine Generation } \\ \text { ZEBRA } & \text { Sodium Nickel chloride battery energy storage } \\ \text { ZSI } & \text { Z-Source Inverter } \\ \text { SLG } & \text { Single Line to Ground } \\ \text { LL } & \text { Line to Line } \\ \text { TPG } & \text { Three-Phase to Ground }\end{array}$




\section{List of Symbols}

The variables that are commonly used in this thesis are provided below. Other variables, only used in specific sections, are defined in the context where they are used.

In general, the variables used in this thesis are represented as follows:

- The sign $\Delta$ followed by a variable represents the small-signal variation of the variable.

- In small-signal analysis, the upper-case letter of a variable refer to the quiescent (dc) component of the variable at the operating point of linearization.

- A variable with a suffix ' $a$ ', ' $b$ ' and ' $c$ ' indicates for the corresponding phase a, phase $\mathrm{b}$, and phase c, respectively.

- A variable with a suffix ' $d$ ' and ' $q$ ' represents its corresponding direct- and quadratureaxis components in the defined synchronous reference frame, respectively.

- The sequence (positive or negative) of a quantity is identified by the sign in the superscript of the quantity.

- A variable with superscript '^’' denotes peak of the variable.

- A variable with subscript ' $r e f$ ' denotes reference value of the variable.

- A variable with subscript 'opt' denotes the optimum/optimal value of the variable.

- A variable with subscript 'ss' denotes the steady-state value of the variable.

$\rho \quad$ Air density

$\lambda \quad$ Turbine's tip speed ratio

$\delta \theta \quad$ Angle difference

$\beta \quad$ Turbine's blade pitch angle

$r \quad$ Radius of turbine's blades

$d \quad$ Damping of natural modes of the generic load model

$P \quad$ Pole pair

G ac gain of the inverter PWM scheme 


\begin{tabular}{|c|c|}
\hline$A$ & Turbine's swept area \\
\hline$\Delta i_{d c}$ & dc-link current ripple \\
\hline$\omega_{s}$ & Stator angular synchronous frequency \\
\hline$\omega_{r}$ & Electrical angular speed of the generator \\
\hline$\omega_{o}$ & Oscillation frequency of the generic load model \\
\hline$\omega_{m}$ & Mechanical angular speed of the turbine \\
\hline$\omega_{g}$ & Mechanical angular speed of the generator \\
\hline$\omega_{e}$ & Stator angular electrical frequency \\
\hline$\omega_{L o}$ & Nominal load-side frequency \\
\hline$\omega_{L}$ & Fundamental angular frequency of the load-side voltage \\
\hline$\varphi_{d s}, \varphi_{q s}$ & $d$-axis, $q$-axis stator fluxes \\
\hline$\varphi_{d r}^{\prime}, \varphi_{q r}^{\prime}$ & $d$-axis, $q$-axis rotor fluxes referred to the stator \\
\hline$\theta_{m}$ & Turbine shaft angle \\
\hline$\theta_{g}$ & Generator shaft angle \\
\hline$\beta_{p}, \beta_{q}$ & Dependence parameters of load powers on frequency \\
\hline$\alpha_{p}, \alpha_{q}$ & Dependence parameters of load powers on voltage magnitude \\
\hline$\alpha_{i}$ & Delay angle of the current source inverter \\
\hline$v_{x y}$ & Output dc voltage of the reduced H-bridge converter \\
\hline$v_{i n v}$ & Input dc voltage of the current source inverter \\
\hline$v_{d c}$ & Output dc voltage of diode bridge rectifier \\
\hline$v_{d}$ & Output dc voltage of the buck converter \\
\hline$v_{c i d}, v_{c i q}$ & $d$-axis, $q$-axis voltage of load-side capacitor filter \\
\hline$v_{\text {bat }}$ & Battery voltage \\
\hline
\end{tabular}




\begin{tabular}{|c|c|}
\hline$v_{W}$ & Wind speed \\
\hline$v_{L d}, v_{L q}$ & $d$-axis, $q$-axis load voltage \\
\hline$v_{L L}$ & Load-side line voltage \\
\hline$v_{L}$ & Voltage across the dc-link inductance \\
\hline$v_{g_{a b c}}$ & 3-phase generator voltage \\
\hline$v_{c_{g d}}, v_{c_{g q}}$ & $d$-axis, $q$-axis voltages across excitation capacitor \\
\hline$v_{c_{g a b c}}$ & 3-phase voltage across excitation capacitor \\
\hline$v_{c_{b}}$ & dc voltage across capacitor of the battery-side LC filter \\
\hline$v_{c_{i_{a b c}}}$ & 3-phase voltages across load-side capacitor filter \\
\hline$v_{L_{a b c}}$ & 3-phase load voltage \\
\hline$n_{\text {gear }}$ & Gear box ratio \\
\hline$m_{i d}, m_{i q}$ & $d$-axis, $q$-axis modulation indices of the current source inverter \\
\hline$m_{i}$ & Modulation index \\
\hline$m_{i_{a b c}}$ & 3-phase modulation indices of the current source inverter \\
\hline$k_{p}, k_{i}$ & Proportional, integral gain of PI regulator \\
\hline$k_{o p t}$ & Optimum torque constant \\
\hline$i_{p d}, i_{p q}$ & $d$-axis, $q$-axis currents of the primary side of the $\Delta / Y_{n}$ transformer \\
\hline$i_{o d}, i_{o q}$ & $\begin{array}{l}d \text {-axis, } q \text {-axis components of the fundamental current of the current source } \\
\text { inverter }\end{array}$ \\
\hline$i_{\text {in }}$ & Input dc current of the reduced $\mathrm{H}$-bridge converter \\
\hline$i_{d s}, i_{q s}$ & $d$-axis, $q$-axis stator currents \\
\hline$i_{d r}^{\prime}, i_{q r}^{\prime}$ & $d$-axis, $q$-axis rotor currents referred to the stator \\
\hline$i_{d c r}$ & Input dc current of the buck converter \\
\hline
\end{tabular}




\begin{tabular}{|c|c|}
\hline$i_{d c g}$ & Generator-side dc current \\
\hline$i_{d c h}$ & Output dc current of the buck converter \\
\hline$i_{d c}^{* *}$ & dc-link current reference set according to generator optimum power \\
\hline$i_{d c}^{*}$ & dc-link current reference set according to load demand \\
\hline$i_{d c}$ & dc-link current \\
\hline$i_{d c, \min }$ & Lower limit of the dc-link current \\
\hline$i_{d c, \max }$ & Upper limit of the dc-link current \\
\hline$i_{\text {bat }}$ & Battery current \\
\hline$i_{L d}, i_{L q}$ & $d$-axis, $q$-axis load currents \\
\hline$i_{p_{a b c}}$ & 3-phase primary-side currents of the $\Delta / Y_{n}$ transformer \\
\hline$i_{o_{a b c}}$ & 3-phase fundamental currents of the current source inverter \\
\hline$i_{g_{a b c}}$ & 3-phase generator current \\
\hline$i_{c_{g d}}, i_{c_{g q}}$ & $d$-axis, $q$-axis excitation capacitor currents \\
\hline$i_{L_{a b c}}$ & 3-phase load current \\
\hline$f_{s}$ & Switching frequency of the converters \\
\hline$f_{r}$ & Resonance frequency \\
\hline$d_{b}$ & Duty ratio of the buck converter \\
\hline$d_{A}$ & Duty ratio of the reduced $\mathrm{H}$-bridge converter \\
\hline$Y_{P}, Y_{Q}$ & Real, reactive power indices of the generic load model \\
\hline$Y_{Q_{p d}}^{-}, Y_{Q_{p q}}^{-}$ & $\begin{array}{l}\text { Power indices of the extended generic load model associated with the negative } \\
\text { sequence current }\end{array}$ \\
\hline$W_{o}$ & Observability Gramian matrix \\
\hline$W_{c}$ & Controllability Gramian matrix \\
\hline
\end{tabular}




\begin{tabular}{|c|c|}
\hline$V_{o}$ & Nominal load voltage \\
\hline$V_{L, \min }$ & Minimum instantaneous voltage applied across the dc-link inductor \\
\hline$V_{L, \max }$ & Maximum instantaneous voltage applied across the dc-link inductor \\
\hline$T_{m}$ & Turbine mechanical torque \\
\hline$T_{e}$ & Generator electromagnetic torque \\
\hline$R_{S}$ & Stator resistance \\
\hline$R_{r}^{\prime}$ & Rotor resistance refereed to the stator \\
\hline$R_{d c}$ & Resistance of the dc-link reactor \\
\hline$Q_{p d o}^{-}, Q_{p q o}^{-}$ & $\begin{array}{l}\text { Nominal powers of the extended generic load model associated with the } \\
\text { negative sequence current }\end{array}$ \\
\hline$Q_{p d}^{-}, Q_{p q}^{-}$ & $\begin{array}{l}\text { Powers of the extended generic load model associated with the negative } \\
\text { sequence current }\end{array}$ \\
\hline$P_{o}, Q_{o}$ & Nominal active, reactive powers of the load \\
\hline$P_{m}$ & Mechanical power captured by the wind turbine \\
\hline$P_{g}, Q_{g}$ & Generator active, reactive power \\
\hline$P_{\text {bat }}^{*}$ & Maximum average power allowed to be absorbed by battery under $i_{d c}^{*}$ \\
\hline$P_{\text {bat }}$ & Battery power \\
\hline$P_{L}, Q_{L}$ & Load active, reactive power \\
\hline$L_{m}$ & Magnetizing inductance \\
\hline$L_{l s}$ & Stator leakage inductance \\
\hline$L_{l r}^{\prime}$ & Rotor leakage inductance refereed to the stator \\
\hline$L_{d c}$ & dc-link inductance \\
\hline$L_{b}$ & Inductance of battery-side LC filter \\
\hline$K_{s e}$ & Shaft equivalent stiffness factor \\
\hline
\end{tabular}




$\begin{array}{ll}J_{m} & \text { Turbine rotor inertia } \\ J_{g} & \text { Generator rotor inertia } \\ J_{e q} & \text { Equivalent rotor inertia } \\ D_{s e} & \text { Shaft equivalent damping factor } \\ C_{r} & \text { Capacitance of ac capacitor filter of current source rectifier } \\ C_{p} & \text { Turbine's performance coefficient } \\ C_{i} & \text { Capacitance of ac capacitor filter of current source inverter } \\ C_{g} & \text { Capacitance of ac excitation capacitor of the generator } \\ C_{d c} & \text { Capacitance of dc capacitor filter of diode bridge rectifier } \\ C_{b} & \text { Capacitance of battery-side LC filter } \\ \bar{v}_{w} & \text { Average wind speed } \\ v_{L d}, v_{L q} & \quad d \text {-axis, } q \text {-axis synchronous frame load voltage (under balanced load) }\end{array}$




\section{Chapter 1}

\section{Introduction}

Recently, utilization of wind energy has achieved a rapid growth in Europe, North America and Asia. Global Wind Energy Council reported that the total capacity of grid-tied wind energy, installed in 2015 alone, exceeded 63GW worldwide [1] . Small-scale wind turbines (WTs), mainly employed in off-grid applications, have also received a lot of demand worldwide [2] . In 2014, the U.S. department of energy (DOE) reported installed capacity of 2.8 MW for small WTs in the United States and exported capacity of 11.2 MW to the global market [3]. In 2015, Canada installed around 1.5 GW of new wind capacity in 36 projects, 23 of which were deployed to serve off-grid communities, as well as municipal or local ownership [4] . Asia is still the largest regional market for wind energy. In China alone, the target is to reach $200 \mathrm{GW}$ of wind power capacity by 2020 [5]. A considerable percentage of this target capacity is expected to be off-grid, due to grid connection issues.

\subsection{Research Motivations}

According to Global Off-Grid Lighting Association, over 25\% of the world's population, mostly in developing countries, has no access to electricity [6]. Even in modern countries, there are remote communities where connection to the main grid is either too expensive or impractical. For example, there are around 175 off-grid communities throughout Canada [7]. Employing renewable energy sources is the most suitable solution for off-grid applications, if intermittency is compensated for. At present, standalone small WTs, ranging in power rating from a few hundred watts to a hundred kilowatts, provide a very attractive renewable energy source for remote communities. These WTs help in reducing the stress on the grid by supplying part of the demand without involving the grid, diminish the air pollution [8] and save on fuel cost by reducing or even eliminating the need for diesel generators, which consume a lot of air-polluting fossil fuels, have high operating and maintenance costs, and may require significant additional costs if installed in a remote area, where fuel transportation and refueling is a complicated mission [9]. Moreover, standalone WTs can be installed wherever wind resource is adequate and there is no access to the grid, or connection to the grid is very costly [10], not permitted or difficult due to official approval requirements. In addition to 
remote communities, standalone small WTs can be used to supply power to boats, recreational vehicles, cottages, local schools, farms, and small manufacturing facilities.

Although the main principles of operation are the same in on-grid and off-grid wind energy conversion systems, the absence of grid in the latter case adds to the hardware and control requirements. In spite of the fact that wind energy is intermittent and cannot be dispatched to meet the assigned commitment, connection to the grid allows for extracting the maximum available power from wind resources at any moment of time. In contrast, for an off-grid Wind Energy Conversion System (WECS) to satisfy the time-varying power demand and maintain balance of power, an energy storage unit is required to compensate for the power deficit and absorb the excess power generated from wind. Another issue with off-grid WECS is the reactive power required by some generator types that has to be supplied by a reactive power source such as a capacitor bank, synchronous condenser, SVC or STATCOM [11].

To date, voltage-source inverter (VSI) is the dominant topology in both large and small-scale WECS. Current-source inverter (CSI), on the other hand, has been adopted mainly in mediumvoltage, high power applications. The advantages reported in the literature for CSI, when substantiated, can make CSI a promising option and possibly a preferred choice for small-scale standalone WECS. Motivated by the huge demand for off-grid small-scale wind turbines and potential of CSI to be employed in such turbines, the research presented in this thesis intends to investigate the feasibility of CSI-based WECS for off-grid applications.

\subsection{Literature Review}

Compared to fixed-speed WTs, variable-speed WTs produce more energy from the same wind resource, with less power fluctuations and lower mechanical stress.

Fixed-speed WTs, in general, use squirrel-cage induction generator, with no power electronic interface [12]-[14]. On the contrary, variable-speed wind turbines enjoy a rather wide range of options for appropriate generator and power converter types. This section provides a review on variable-speed WECSs from generator and converter viewpoints. The section starts with a brief historical data. 


\subsubsection{History of Wind Energy}

Wind is a renewable energy source that has been used for a long time in water pumps, wheat mills and sailing ships. Since1980s, wind has been recognized as an efficient and reliable source for generating electricity [15]. In the recent years, the utilization of small wind turbines (SWTs), mainly adopted in off-grid projects, has grown all over the world and is expected to become more desirable in the future, especially with the development of energy storage technologies with the required capabilities. Fig. 1.1 illustrates the global annual installed SWT since 2009 and forecasts the annual growth rate until 2020 [2]. According to the figure, the market of SWT could subsequently feature a steady growth rate of 20\% from 2015 to 2020. By 2020 , a cumulative installed capacity of approximately 2 GW is expected to be achieved.

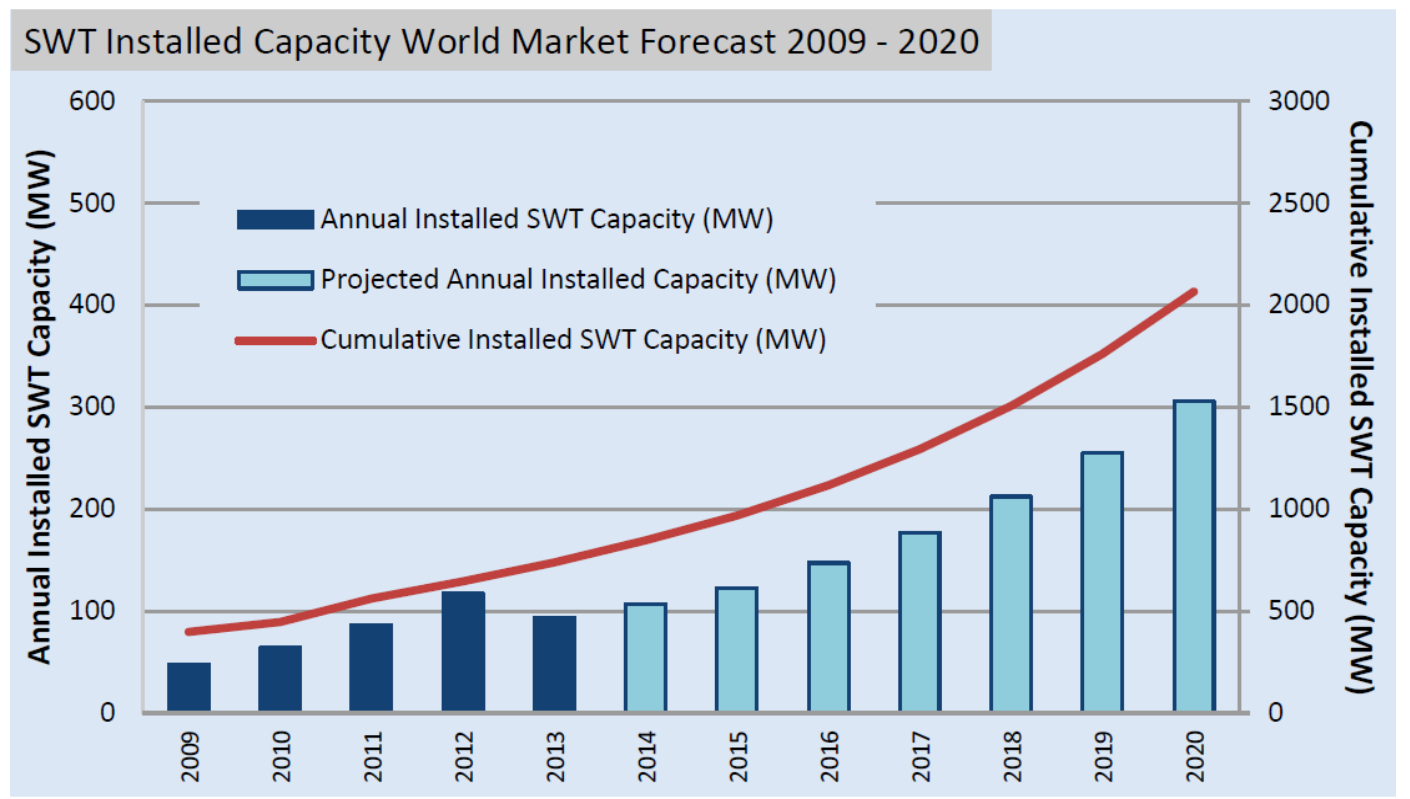

Fig. 1.1: Global annual installed Small Wind turbine (SWT)[2].

\subsubsection{Conventional and Potential Generator Types used in WECS}

The following generator types have been employed in the existing wind energy conversion systems or have been reported in the literature:

1. Wound-Rotor Induction Generator (WRIG)

2. Doubly-Fed Induction Generator (DFIG)

3. Brushless Doubly-Fed Induction Generator (BDFIG) 


\section{Brushless Doubly-Fed Reluctance Generator (BDFRG)}

5. Squirrel-Cage Induction Generator (SCIG)

6. Wound-Rotor Synchronous Generator (WRSG)

\section{Permanent-Magnet Synchronous Generator (PMSG)}

8. Permanent-Magnet Induction Generator (PMIG)

9. Switched-Reluctance Generator (SRG)

Fig. 1.2 shows a simplified configuration for a standalone, variable-speed WRIG-based WECS. The stator is connected to the PCC (point of common coupling), while the rotor is connected to a combination of a fixed resistance $\left(R_{\text {ext }}\right)$ and a power electronic converter that emulates an adjustable resistance. By varying the value of the resistance seen by the rotor windings, the generator can run at different operating points. A soft starter is needed in order to reduce the inrush current at start-up [12]. Standalone WRIG is simply controlled to produce stable voltages with constant amplitude and frequency even though rotor speed is varied by several percent [16]-[17]. Due to limited range of speed variation, WRIG has been used for a long time in fixed- speed WTs, rather than variable-speed WTs [18] .

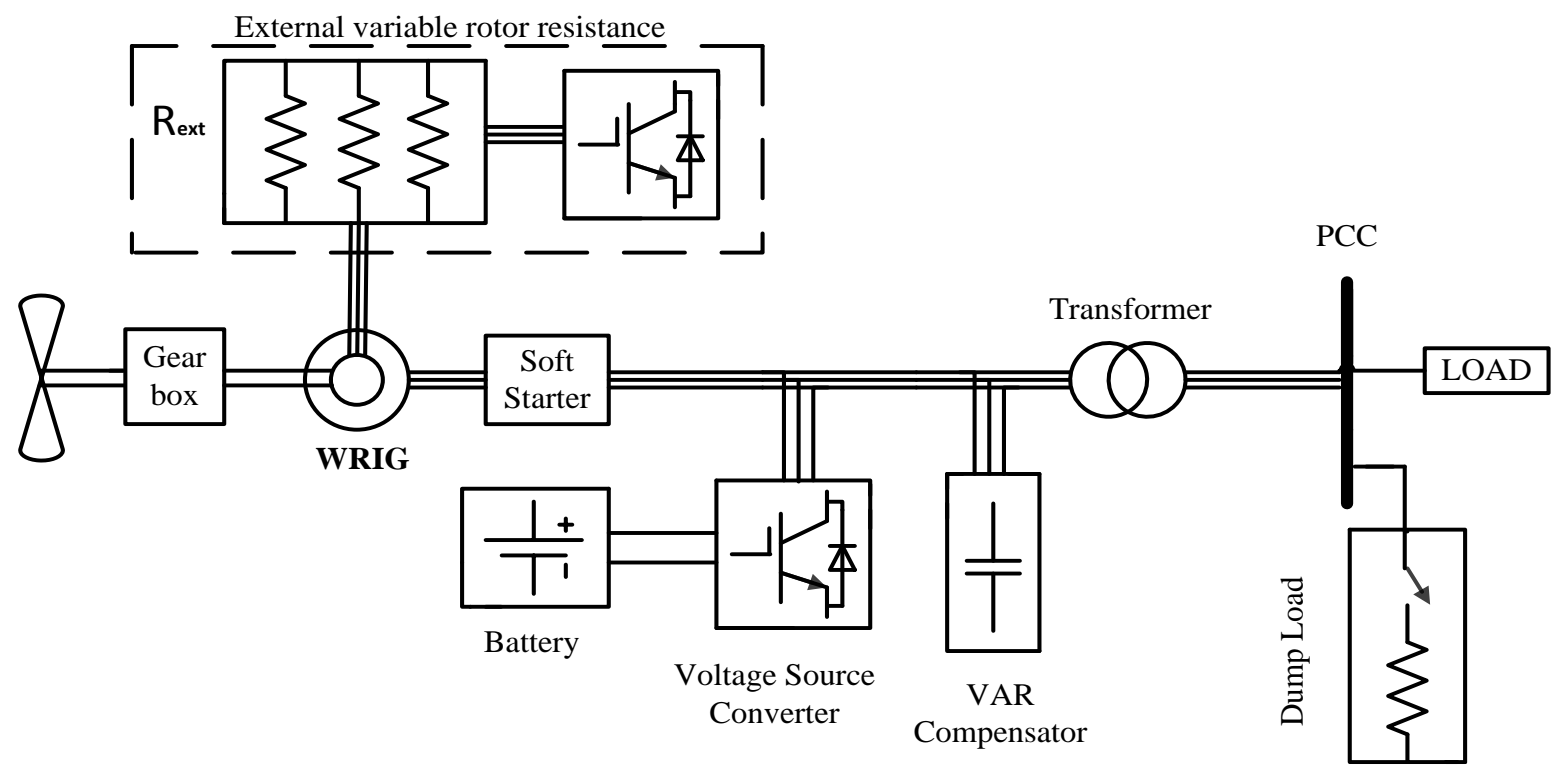

Fig. 1.2: WRIG-based standalone WECS. 
A DFIG-based standalone WECS is constructed by connecting the stator directly, and the rotor via a power electronic converter, to the PCC, as shown in Fig. 1.3. The flow of power through the stator is unidirectional, while the direction of power flow through rotor depends on the operational mode of generator. If the generator is operating below synchronous speed, the power is received by the rotor. If the generator is operating above synchronous speed, the rotor delivers power. The main advantage offered by DFIG is that its rotor power converter is rated only at $30 \%$ of the stator power [15]. This feature makes DFIG a preferred choice in highpower grid-connected WECSs, due to the huge economic gains resulting from reduced sizes of power converters and filters [19]. Moreover, different control strategies, developed and investigated in [20]-[22], have demonstrated ease of control of DFIG in standalone wind energy applications, especially from the voltage regulation point of view. However, the rotor voltage and current need to be carefully controlled during the initial transients, as they can be too high to be handled by the reduced-size converters [23].

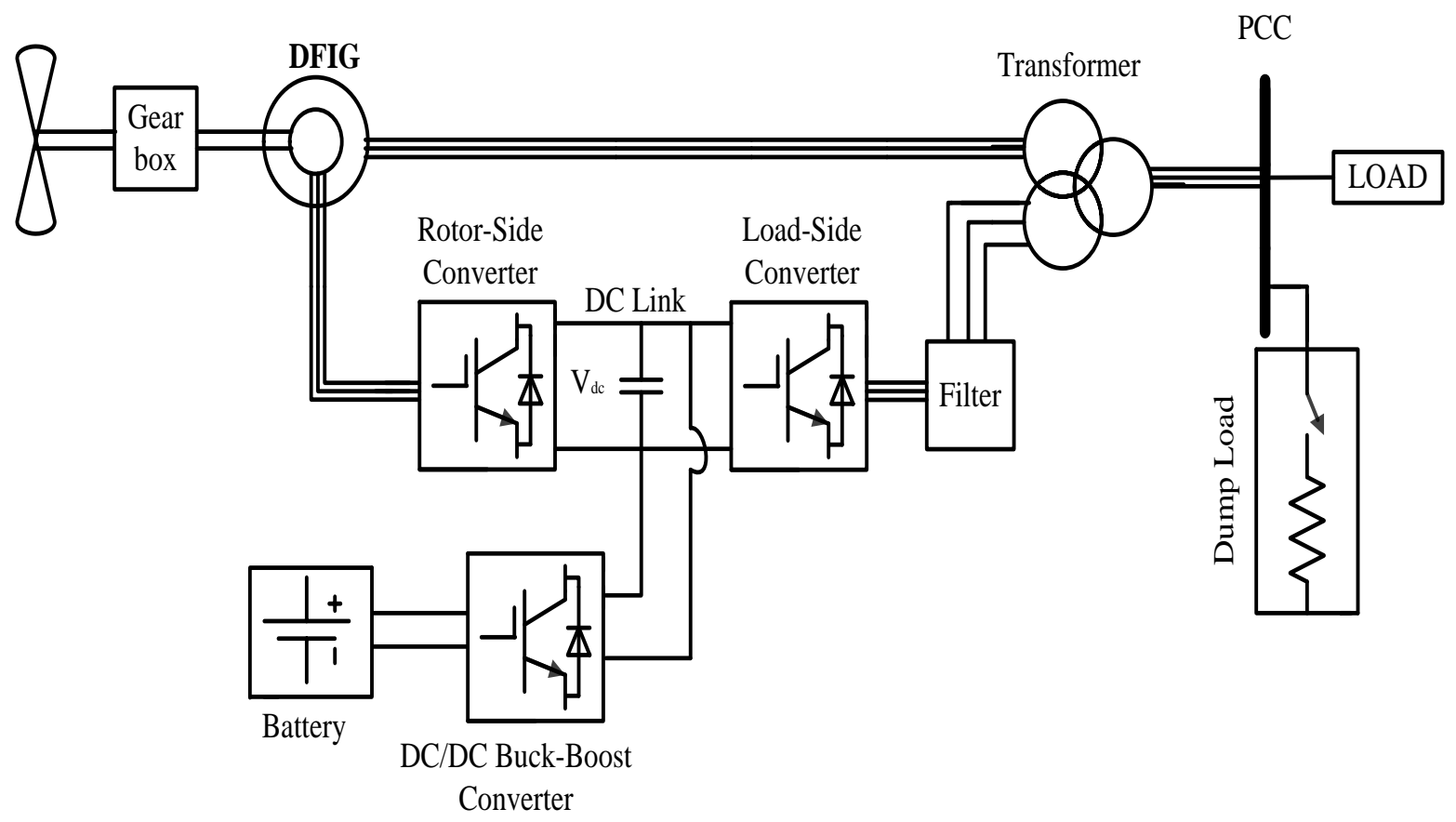

Fig. 1.3: DFIG-based standalone WECS.

BDFIG consists of two cascaded wound-rotor induction machines, one for power generation and the other one for control [24]. BDFIG has two groups of stator windings referred to as power winding $(\mathrm{PW})$ and control winding $(\mathrm{CW})$. As shown in Fig. 1.4, the PW is directly 
connected to the PCC, while the CW is connected to the PCC through two back-to-back reduced-size power converters, i.e., machine-side converter (MSC) and load-side converter (LSC). BDFIG's benefits are similar to those of DFIG [24] . Nevertheless, its size is larger, and the complexity of its assembly and control is higher [19], for the same power rating. Despite these disadvantages, BDFIG is still attractive for large grid-connected WTs, especially for off-shore applications, where WTs have to be very reliable and nearly maintenancefree [25],[26].

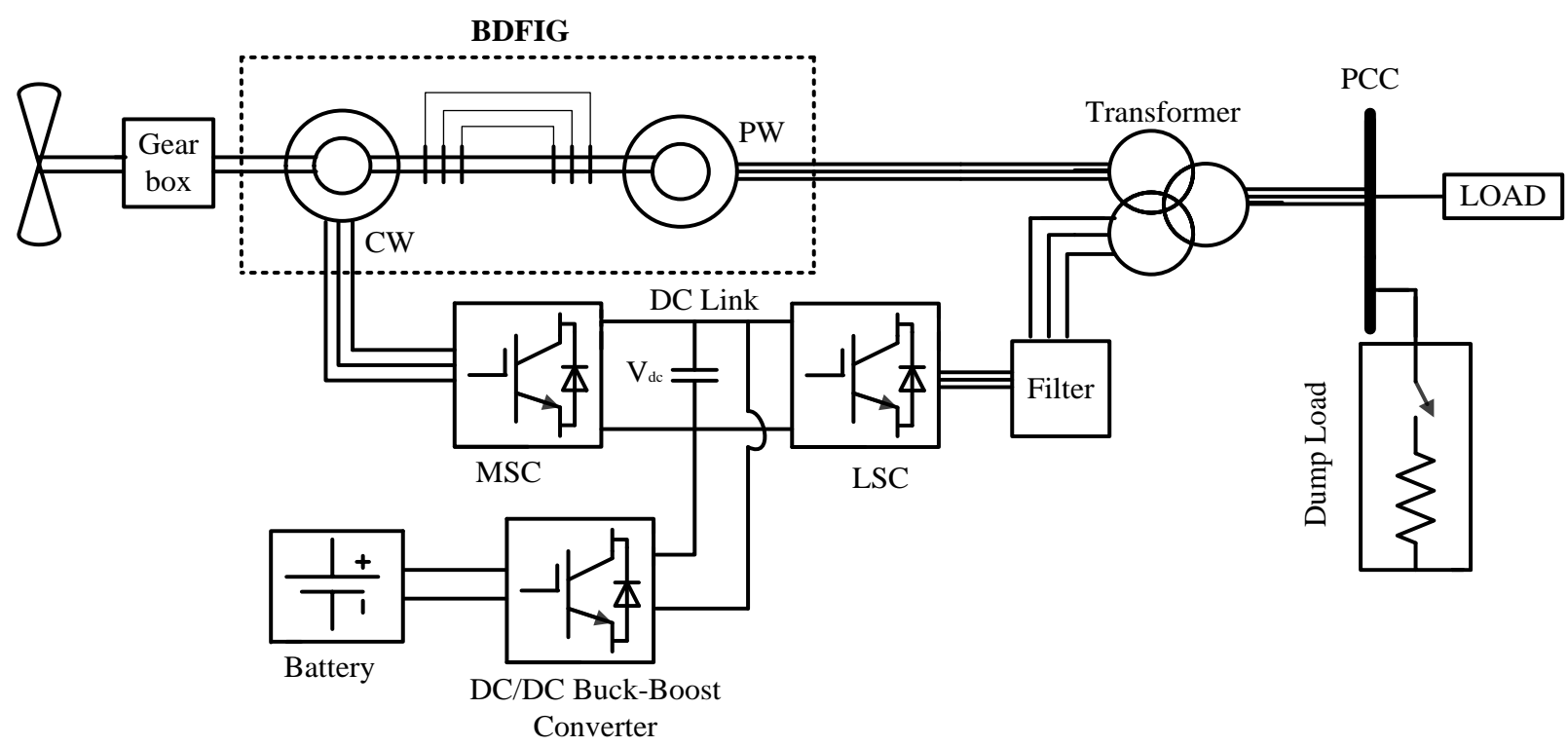

Fig. 1.4: BDFIG-based standalone WECS.

BDFRG is a different design featuring a reluctance rotor instead of a wound rotor in BDFIG. Although BDFRG is more efficient and reliable than BDFIG, it still has a complex rotor design and a large size due to a smaller torque-volume ratio [27]. However, recent improvements in reluctance rotor design may result in higher future interest in the BDFRG [28].

Amongst the traditional induction generators, SCIG is the smallest in size, lowest in cost and most robust in structure [29],[30]. As a mature machine in wind energy applications, SCIGbased WT systems have been of interest in many research projects, including simulator design, emulator set-up, novel power converters as well as control schemes, self-excitation and voltage build up techniques in standalone applications [31]-[37]. Since SCIG is one of the highly 
recommended generators for off-grid WTs, its possible configurations will be discussed in detail in chapter 2 (subsection 2.1.1).

WRSG requires a dc excitation, which can be provided by either an external dc source through slip rings and brushes or a brushless exciter, involving power electronics and an auxiliary ac generator. Fig. 1.5 presents a typical standalone WECS based on WRSG. The generator-side converter (GSC) is responsible for Maximum Power Point Tracking (MPPT), while the load-side converter (LSC) controls the voltage and frequency at PCC, provided that the DC link voltage is regulated by implementing a power management strategy that controls the power transactions of battery and dump load under different load and wind speed conditions. WRSG-based standalone WECS has been mentioned in [38] as a promising alternative for serving remote load demands. Different control schemes for stator voltage regulation in standalone WRSG are described in [39].

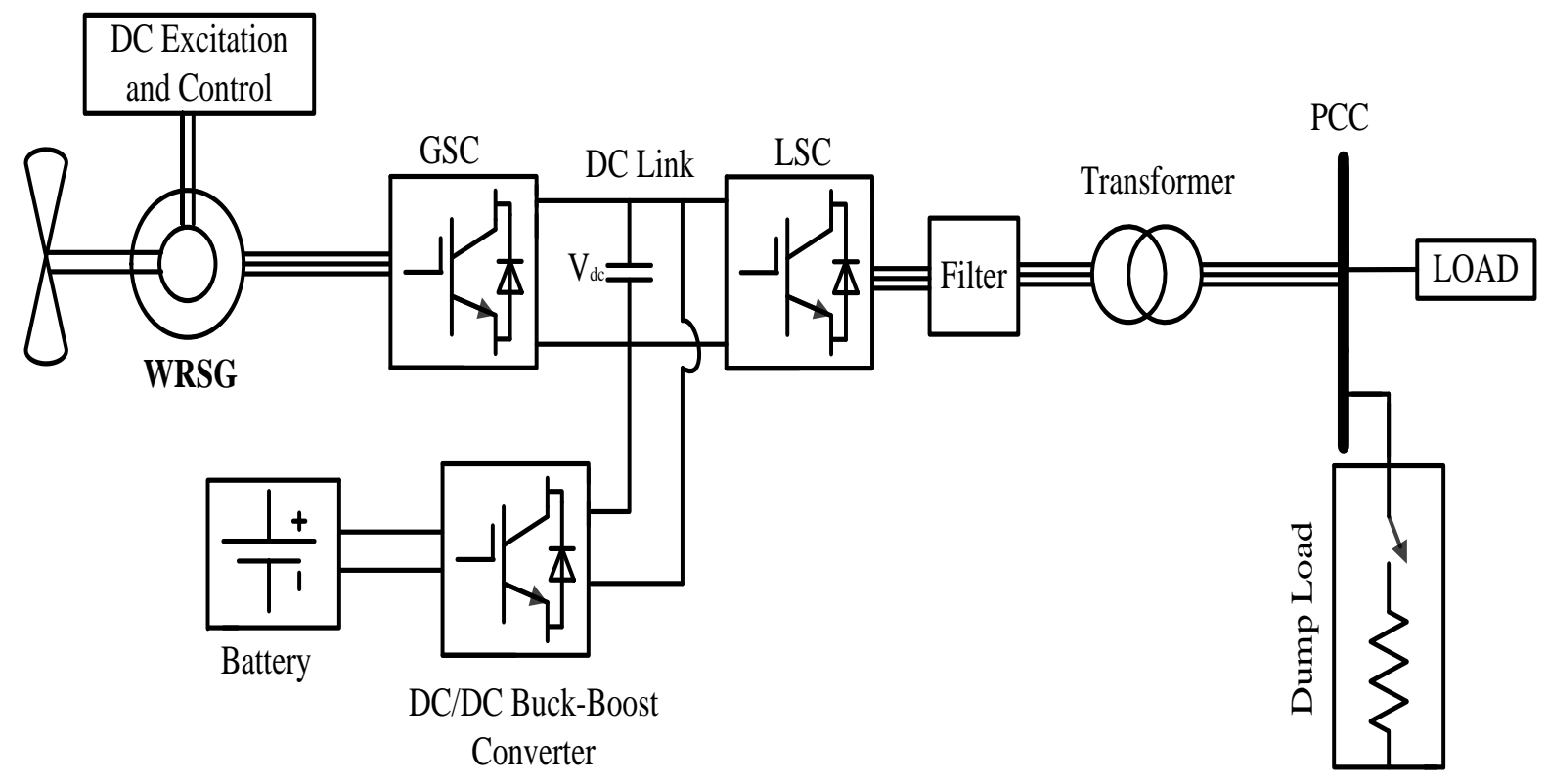

Fig. 1.5: WRSG-based direct-drive standalone WECS.

Unlike WRSG, PMSG is a brushless self-excited synchronous machine. Since 1996, PMSG has become more attractive than WRSG due to a decrease in the costs of permanent magnets and power converters [40]. At present, PMSG is known to be the prominent solution in directdrive, small-scale standalone WTs [22],[41]-[49]. Therefore, its possible configurations will be discussed in detail in chapter 2 (subsection 2.1.1). 
By adding improved power factor and better performance to the advantages of the SCIG, PMIG, a relatively new induction generator-based machine, has a very good potential to serve as a direct-drive generator in grid-connected [50],[51] and isolated WECS [52]. Although PMIG has been considered for direct-drive WTs since 1999 [53], it has just recently been recognized in wind energy market. Due to its improved power factor and efficiency, some manufacturers [54],[55] have started considering PMIG as a good alternative for the highefficiency PMSG, especially for small-scale WECSs. Therefore, its possible configurations will be discussed in detail in chapter 2 (subsection 2.1.2 ).

SRG is a structurally simple and robust machine. Its stator and rotor are usually made of steel laminations. The stator consists of a number of salient poles with windings concentrated around them. The rotor consists of a number of salient poles and has neither windings nor permanent magnets. Fig. 1.6 shows a typical standalone WECS using direct-drive SRG. The machine is normally driven by an Asymmetric Half Bridge Converter (AHBC) [56][57]. As a simple, robust, reliable and inexpensive machine with flexible control, SRG has shown a good potential to serve as a direct-drive generator in standalone WECS [56],[57] as well as gridconnected WECSs [58]-[60].

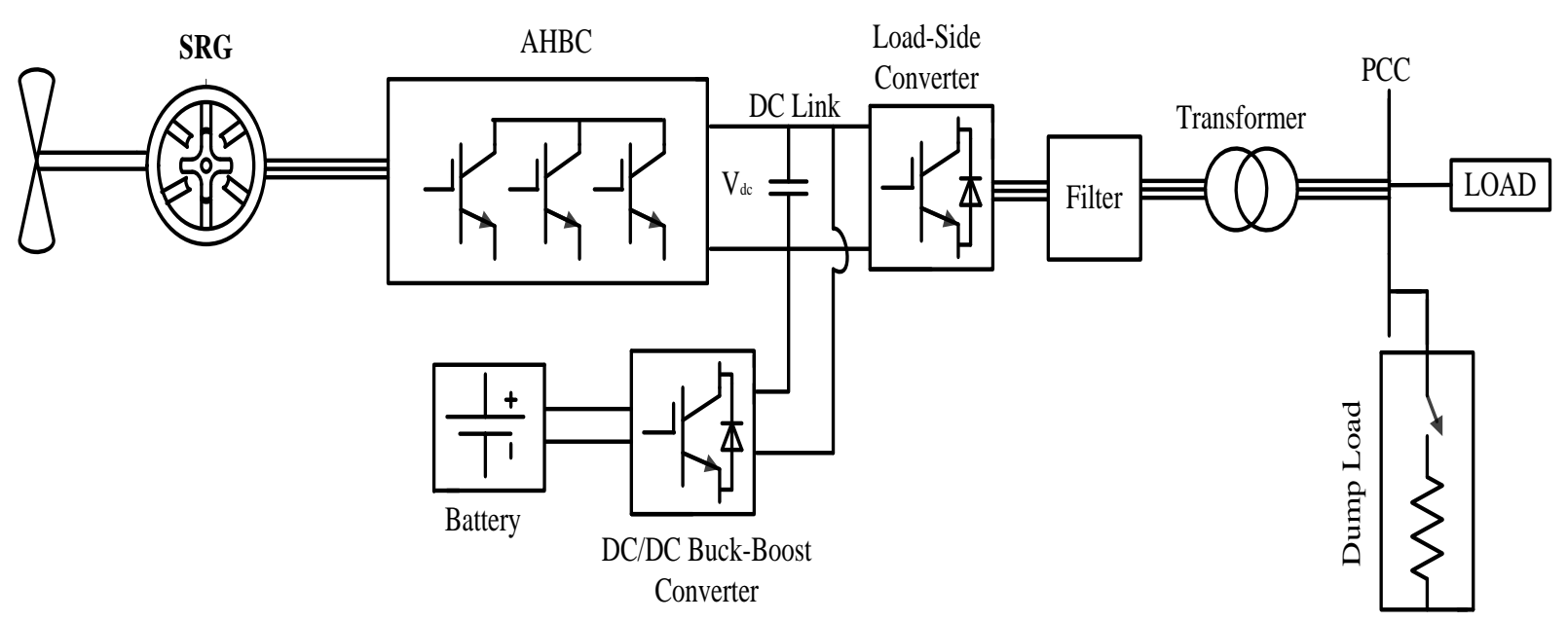

Fig. 1.6: Standalone WECS using direct-drive SRG. 


\subsubsection{Power Electronic Converter Topologies for Standalone WECS}

Currently, voltage-source converter (VSC) is the prominent converter type in WECSs. However, there are other power electronic configurations deployed in WECSs. This subsection gives an overview of the power electronic converter topologies that are commonly used, or have the potential to be used, in standalone WECSs.

\section{A) Diode Rectifier Bridge + DC/DC Boost Converter + VSI}

A three-phase diode bridge rectifier and a voltage-source inverter (VSI) with dc link capacitor in between, is shown in Fig. 1.7. Since the diode rectifier is an uncontrolled converter, a dc booster is used to achieve maximum power point tracking (MPPT). A buck-boost dc/dc converter may also be used to handle the extra power available under high wind speed conditions [61]. Diode rectifier is a simple and cost-effective solution, especially for a permanent magnet generator-based WECS [62],[63]. The same topology can also be used in an induction generator-based WECS, but it is less attractive due to the need for an external source of VAR. Employing semi-controlled rectifiers is also a possibility [64]. One problem with using a diode rectifier as the generator-side converter is the resulting distortion in the stator current waveforms, leading to higher losses and torque ripples in the generator.

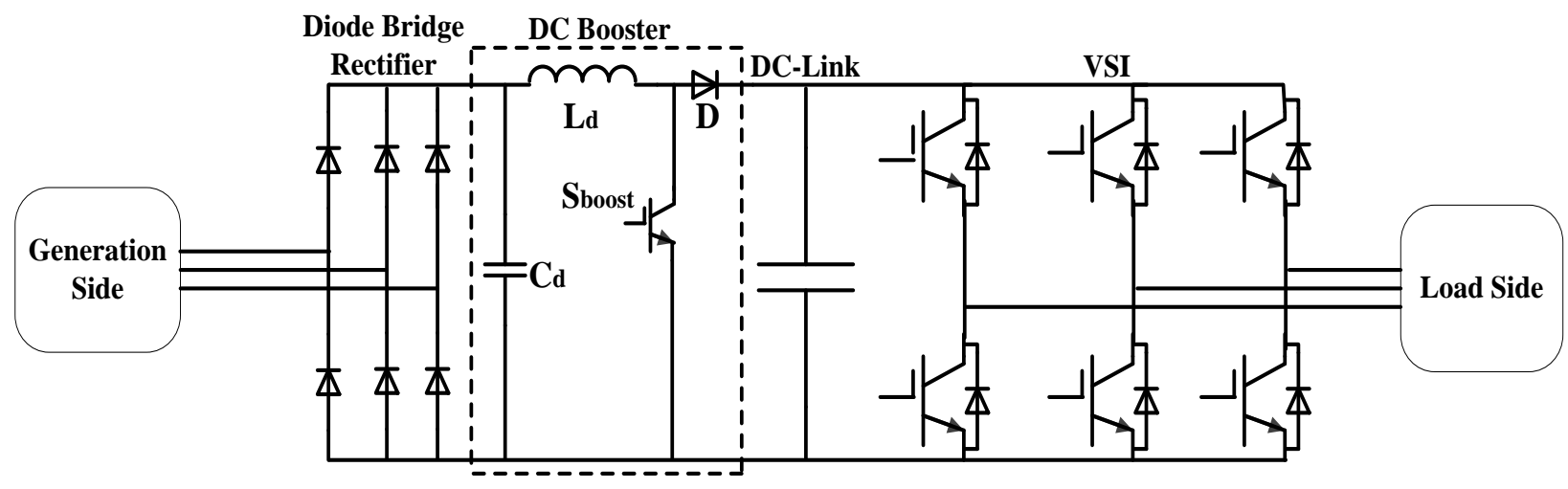

Fig. 1.7: Diode rectifier + dc/dc boost converter + VSI.

\section{B) Two-Level Back-to-Back Voltage-Source Converter (2L-BTB-VSC)}

The drawbacks of using a diode rectifier as the generator-side converter are avoided in the 2L-BTB-VSC system illustrated in Fig. 1.8, where rectification is done by a voltage-source rectifier (VSR). Compared to the system shown in Fig. 1.7, 2L-BTB-VSC provides more 
efficient MPPT control, but at a higher cost and control complexity [61]. Indeed, the 2L-BTB VSC is a well-established technology for WECSs [27],[63], especially in low-voltage, smallpower WT systems, and thus a mature solution for standalone WECSs.

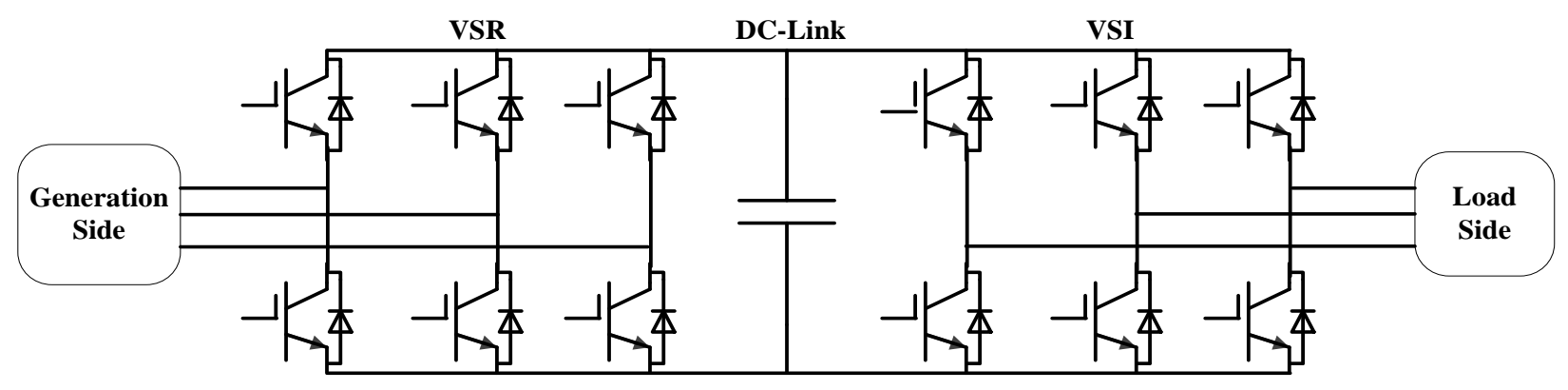

Fig. 1.8: Two-level, back-to-back voltage-source converter topology.

\section{C) Multi-Level Converter}

As the power and voltage levels increase, multi-level (ML) converters are preferred. Compared to the 2L-BTB, ML converters, especially three-level converters, produce much lower switching losses, as well as lower switch stress, harmonic distortion and $d v / d t$ stress on the generator and transformer [65],[66]. However, due to higher number of switches, higher cost and control complexity are associated with ML converters. In a standalone WECS, the use of ML converters may not be justified as the levels of voltage and power are generally low. The details of classical and advanced converter topologies have been covered in the literature [67]-[69].

\section{D) Current-Source Converter}

Although VSCs dominate the present WECS market, due to their well-developed technology and fast dynamic response, current-source converter (CSC) can also be a good alternative. CSC can be constructed as BTB and ML converters. A BTB-CSC is shown in Fig. 1.9. CSC offers some advantages over VSC [70],[71]. However, CSC has a number of drawbacks that need to be taken care of in order to make CSC an effective solution [71],[72].

CSC is well-established for high power applications such as medium-voltage industrial drives. Therefore, references [73],[74] have proposed the employment of CSC in Mega-Watts on-grid WECSs. However, the use of CSC for a small-scale off-grid WECS has not been proposed yet, although CSC can also offer some advantages in such an application. 


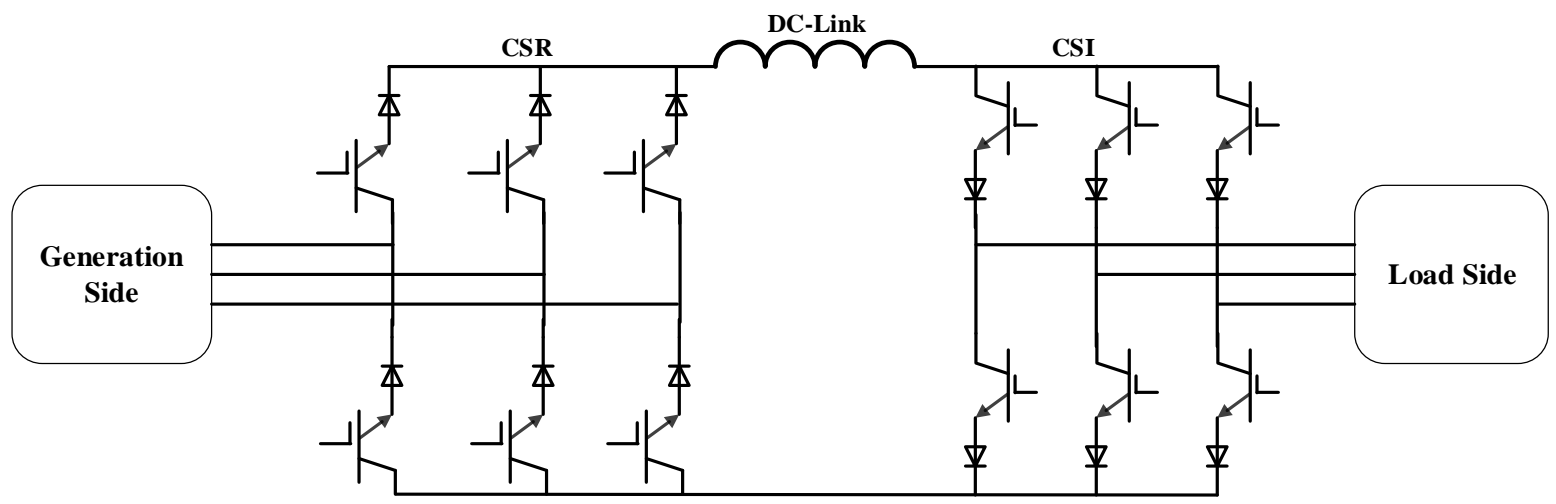

Fig. 1.9: Back-to-back current-source converter topology.

\section{E) Diode Rectifier Bridge + Impedance-Source Inverter}

Impedance-source inverter or Z-source inverter (ZSI) was first proposed in 2003 [75]. A converter system composed of a three-phase diode bridge rectifier and a ZSI is shown in Fig. 1.10. Unlike traditional VSI or CSI, ZSI provides the buck-boost feature without an extra $\mathrm{dc} / \mathrm{dc}$ buck-boost converter. Moreover, dead times and overlap times, required to prevent short and open circuit conditions in VSI and CSI, respectively, are not concerns in ZSI.

Compared to the three-stage conversion system shown in Fig. 1.7, number of switches is reduced by one in the two-stage conversion system shown in Fig. 1.10.

References [76],[77] have shown the potential of ZSI to replace the conventional VSI with dc booster in standalone WECSs. In [78], a buck-boost dc/dc converter was used to integrate a storage battery unit with a ZSI-based WECS through one of the ZSI's capacitors.

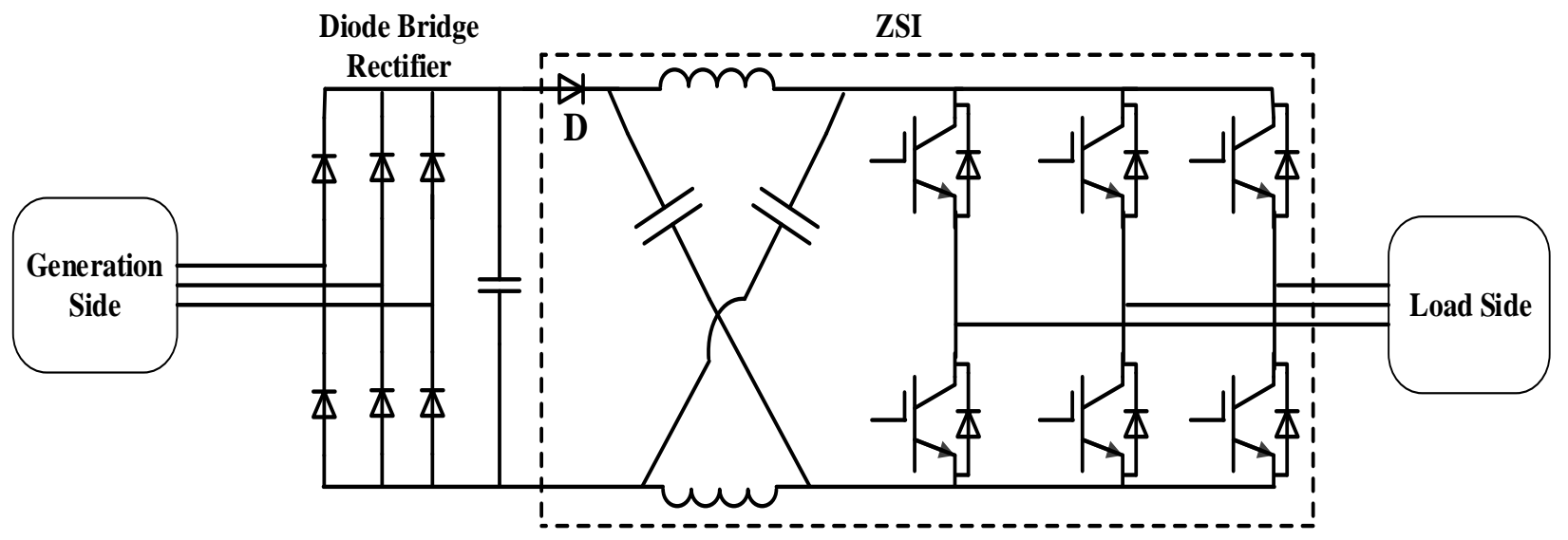

Fig. 1.10: Diode Rectifier Bridge + Z-Source inverter. 


\section{F) Matrix Converter}

The presence of bulky dc-link capacitor and inductor in VSC and CSC topologies reduces their efficiency and shortens their overall lifetime. On the contrary, matrix converter (MC), which is a direct ac-ac converter, has a higher efficiency, longer life and reduced size due to the absence of dc-link energy storage devices [79]. The configuration of a matrix converter, using nine bi-directional switches, is shown in Fig. 1.11. The bi-directional switches allow voltage blocking and current conduction, irrespective of voltage polarity and current direction. The switches are controlled in order to produce the desired output voltage magnitude and frequency on the load side. The output frequency of MC is unrestricted (limited only by the switching frequency), but its output voltage magnitude is limited to 0.866 of that of the input voltage.

One of the challenges in MCs is safe commutation in the absence of freewheeling paths. Connecting two input lines to the same output line causes a short circuit on the input side (i.e., generation side), whilst disconnecting one of the output lines causes an open circuit on the output side (i.e., load side), causing over-voltage spikes. Therefore, safe commutation of MC has triggered a good deal of research activity [80],[81].

In 2001, a novel MC topology, free of the commutation problems, was proposed by Wei and Lipo [82]. Such a converter is known as indirect matrix converter (IMC). The idea of this twostage converter is based on a fictitious dc link, although no energy storage element exists between the supply- and load-side converters.

The employment of MC in an on-grid SCIG-based WECS has been proposed by [83]. IMC has been used in an on-grid DFIG-based WECS in [84]. Although the use of MC and IMC in a small-scale off-grid WECS can be a possibility, the commutation problems in MC need to be carefully addressed, and the absence of dc link component, in both configurations, complicates the integration of an electrical energy storage system. 


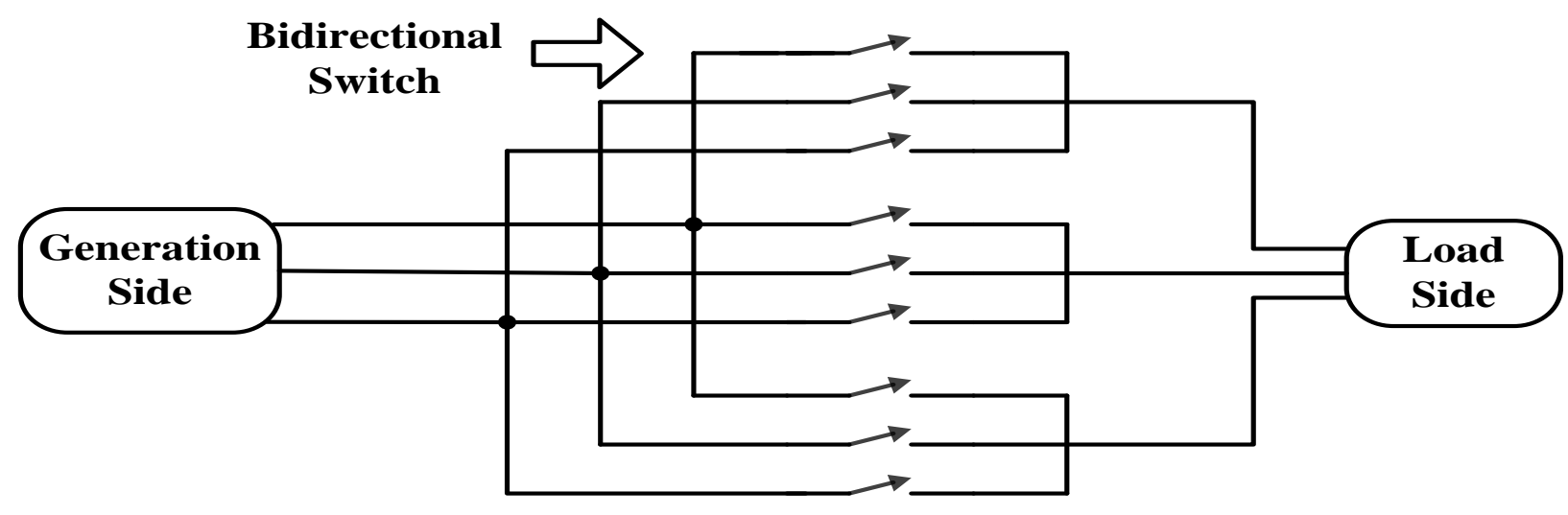

Fig. 1.11: Matrix converter.

Amongst the power converters which have been employed or have the potential to be employed in WECSs (i.e., VSC, CSC, ZSI, MC and IMC), VSC is the dominate topology in both large and small-scale WECSs [63]. The potential of an impedance-source inverter, as a replacement for the conventional VSI-dc booster combination in standalone WECSs, has been demonstrated in [76]-[78]. Although the use of MC and IMC, in a small-scale off-grid WECS, is a possibility, the commutation problems in MC need to be carefully addressed, and the absence of dc link component, in both configurations, complicates the integration of an electrical energy storage system. CSC is a reliable technology, but has been mainly proposed and validated for Mega-Watts-level on-grid WECSs [73],[74]. However, the advantageous features reported in the literature for CSC, when substantiated, can make CSC a promising option for small-scale standalone WECSs.

In the following subsection, the advantages of pulse-width modulated current-source inverter (PWM-CSI) over pulse-width modulated voltage-source inverter (PWM-VSI), for a smallscale WT, are highlighted.

\subsubsection{PWM-CSI versus PWM-VSI for Standalone WECS}

This subsection conducts a comparison based on reliability, cost, efficiency, and protection requirements, between PWM-CSI and PWM-VSI for small-scale standalone WECS, assuming that the inverters use six IGBT switches with six antiparallel diodes (VSI) or series diodes (CSI), feed the same three-phase load at the same voltage and frequency, have comparable 
ratings and operate at the same switching frequency and under the same environmental conditions.

\section{A) Reliability}

Reliability of a power electronic converter is usually measured in terms of the rate of failures leading to converter malfunction [85]. These failures are mainly related to the switching semiconductor devices, capacitors, inductors and transformers. According to a study reported in [86], 34\% of power electronic system failures are related to semiconductor devices. These results are consistent with those of another study reported in [87], carried out by 295 different industrial sectors, showing that semiconductor power devices are the most fragile components of power electronic converters.

Failures of an IGBT are classified into two classes: open-switch fault (OSF) and short-switch fault (SSF). OSF can be caused by bond wire lift or rupture [88] and gate drive failure [89]. $\mathrm{SSF}$, on the other hand, can be caused by bond wire rupture, impact ionization, collector overcurrent, and gate circuit degradation [90]. Moreover, dynamic avalanche of antiparallel diode can cause SSF in IGBTs [91]. In VSI, SSF is fatal and can lead to potential destruction of the failed IGBT, the remaining IGBTs, and other components. On the contrary, SSF is not a serious issue in CSI, but it can degrade the inverter's performance. On the other hand, OSF is not fatal for VSI, while it is a critical issue in CSI, because it can lead to dc-link current interruption, producing high overvoltage transients, and destruction of the failed IGBT as well as the remaining IGBTs.

Similar to IGBT, a power diode can fail as open-switch or short-switch. The open-switch failure mode is mainly caused by bond wire lift or rupture, while the short-switch failure mode can take place as a result of static high voltage breakdown, rise of leakage current, snappy recovery, reverse recovery dynamic avalanche, and high-power dissipation [92].

Considering the number of causes of faults mentioned above, one can conclude that in IGBTs and diodes, the probability of short-switch failure mode is higher than that of open-switch failure mode. SSF in IGBT or its antiparallel diode is fatal in VSI, thus diminishing its reliability significantly. On the contrary, SSF does not adversely affect the reliability of CSI unless it lasts for a long period of time. 
The second most fragile component in a power electronic converter is capacitor [85]. The results of the study reported in [87] suggest that capacitors are responsible for $18 \%$ of power electronic converter failures, while only $5 \%$ of failures are related to inductors. Therefore, the impact of high failure rate of dc-link capacitor on the reliability of VSI is much higher than that of low failure rate of dc-link inductor on the reliability of CSI.

Based on the above analysis, one can conclude that IGBT-based CSI is potentially more reliable than its counterpart, IGBT-based VSI.

\section{B) Cost}

Due to the use of series diodes in IGBT-based CSI, it is expected that the cost of the system be higher than that of IGBT-based VSI. For cost comparison, Table 1.1 shows the prices for two 30kW-PMSG-WECSs, designed for grid connected applications, using VSI and CSI, respectively [93]. Even though the capital cost of CSI-based system is higher than that of the VSI-based system, the difference in the cost is not significant due to lower filtering requirements of CSI. Unlike PWM-VSI, which requires an L-C or L-C-L filter, only a capacitor filter is required in CSI. Moreover, the lifetime of the dc-link inductor in CSI is much longer than that of the dc-link capacitor in VSI [71]. This can result in lower operation and maintenance (O\&M) costs for CSI-based WECS. Overall, capital cost of CSI is slightly higher than that of VSI, but its O\&M costs are lower.

Table 1.1: Cost Comparison of 30kW PMSG-based Wind Turbines with VSI and CSI [93].

\begin{tabular}{lcc}
\hline \multicolumn{1}{c}{ Component } & IGBT-based PWM-VSI & IGBT-based PWM-CSI \\
& US\$ & US\$ \\
\hline Blades (3, Horizontal axis) & 2,145 & 2,145 \\
Generator & 18,015 & 18,015 \\
Controller + rectifier & 3,204 & 3,204 \\
Inverter (including filter) & 10,987 & 11,583 \\
\hline \multicolumn{1}{c}{ Total } & $\mathbf{3 4 , 3 5 1}$ & $\mathbf{3 4 , 9 4 7}$ \\
\hline
\end{tabular}

\section{C) Efficiency}

In a VSI, the dc-link capacitor has a small Equivalent Series Resistance (ESR) and carries only the ripple components of current at steady state, whereas in a CSI the dc-link inductor has 
a larger ESR and carries both dc and ripple components of the dc-link current. Moreover, since there are always four devices (i.e., two IGBTs and two diodes) conducting in a CSI, its conduction energy losses are expected to be higher than that of VSI over a specified period of time. Quantitative analysis performed by [94] has shown that conduction losses of CSI can be more than 2 times higher than those of VSI. It is noteworthy that conduction losses in CSI can be reduced if the dc-link current is reduced according to reduction in demand or if reverseblocking (non-punch-through) IGBTs, that do not need series diodes, are employed. The quantitative analysis in [94] shows that switching losses of a CSI are only $30 \%$ of those of a VSI of comparable ratings. This can be justified by the high commutation voltages experienced by the VSI switches during turn-on and turn-off processes. Overall, compared to CSI, VSI features a higher efficiency.

\section{D) Protection Requirements}

In sinusoidal PWM VSI, the peak value of the fundamental component of ac-side line voltage can be expressed as

$$
\widehat{V}_{L L, 1}=\frac{\sqrt{3}}{2}\left(m_{i}\right)\left(v_{d c}\right)
$$

where $v_{d c}$ is the dc-link voltage and $m_{i}$ the modulation index $\left(0<m_{i} \leq 1\right)$. To guarantee a constant $\widehat{V}_{L L, 1}$, the constraint on the dc-link voltage is given as

$$
v_{d c} \geq \frac{2}{\sqrt{3}} \widehat{V}_{L L, 1}=2 \widehat{V}_{\emptyset, 1}
$$

Similarly, in sinusoidal PWM CSI, the peak value of the fundamental component of ac-side line current can be expressed as

$$
\hat{I}_{L, 1}=\frac{\sqrt{3}}{2}\left(m_{i}\right)\left(i_{d c}\right)
$$

where $i_{d c}$ is the dc-link current. Thus, the constraint on the dc-link current is given as

$$
i_{d c} \geq \frac{2}{\sqrt{3}} \hat{I}_{L, 1}
$$

From (1.2), the dc-link voltage in VSI must be at least twice the peak value of the fundamental component of the ac-side phase voltage, irrespective of the power demand level. In case of a fault at the ac terminals, a huge transient current will flow through the switches that are on at the moment of fault occurrence. Therefore, a current limiter is absolutely 
necessary to protect the switches against overcurrent [95]. In CSI, no current limiter is required because the current is limited by the dc-link current controller, giving the topology an inherent current-limiting feature. On the other hand, the dc side of CSI acts as a current source and hence a fast detection and protection during dc-link current interruption is a must [96]. However, from (1.2) and (1.4), one can notice that unlike the dc-link voltage in VSI, the dclink current in CSI can be reduced if the power demand is reduced. This reduces the consequences of current interruption at low demands.

Based on the comparisons from the viewpoints of capital cost, overall efficiency, and opencircuit fault protection requirements, IGBT-based PWM-VSI is preferred for small-scale offgrid WECS. On the contrary, IGBT-based PWM-CSI is the winner in terms of reliability, O\&M cost, and short-circuit fault protection requirements. The following advantages offered by CSI over VSI need to be considered as well when choosing the converter topology for a small-scale off-grid WECS.

1) CSI has an inherent voltage-boost capability, which is an advantage in WECS application, where the rectified dc voltage is low at low wind speeds. This feature will help in capturing wind power at low wind speeds, thus providing a wider range of operation.

2) In a wind turbine, the gearbox, generator and the associated power converter are usually installed in the nacelle. If the CSI is installed at the bottom of the tower, the length of the connecting cable can help reduce the size and thus cost of the required dc-link inductor.

Even though based on the above discussions, CSI offers high potentials for small-scale $(<$ $100 \mathrm{~kW}$ ) off-grid WECS, its performance in such an application has never been investigated. Therefore, this dissertation focuses on employing CSC in low-power off-grid WECS.

\subsubsection{Energy Storage in Standalone WECS}

For an off-grid WECS to satisfy time-varying power demand and maintain balance of power, an energy storage unit is required to compensate for the power deficit and absorb the excess power generated from wind. Moreover, energy storage improves the quality of power delivered to the varying load. The energy storage technologies that are feasible for wind energy integration are: Flywheel Energy Storage (FES), Pumped Hydro Energy Storage (PHES), Compressed Air Energy Storage (CAES), Super-capacitor Energy storage (SES), Superconducting Magnetic Energy Storage (SMES), Lead Acid Battery (LAB), Nickel 
Cadmium battery Energy Storage (NCES), Lithium Ion battery Energy Storage (LIES), Sodium Sulphur battery Energy Storage (NaSES), Sodium Nickel chloride battery energy storage (ZEBRA), Flow Battery Energy Storage (FBES), and Hydrogen Energy Storage (HES). The details of these storage systems have been covered in the literature [10],[97]-[99].

Table 1.2 summarizes the main advantages and drawbacks of each energy storage technology.

Table 1.2: Advantages and drawbacks of different energy storage technologies.

\begin{tabular}{|c|c|c|c|}
\hline Class & Topology & Advantageous & Drawbacks \\
\hline \multirow[t]{3}{*}{$\begin{array}{l}\text { Mechanical } \\
\text { storage } \\
\text { systems }\end{array}$} & FES & $\begin{array}{l}\text { - High power density } \\
\text { - High efficiency } \\
\text { - Long Lifetime } \\
\text { - Environmentally inert }\end{array}$ & $\begin{array}{l}\text { - Low energy density } \\
\text { - Full self-discharge/day }\end{array}$ \\
\hline & PHES & $\begin{array}{l}\text { - Mature technology } \\
\text { - High capacity } \\
\text { - Low capital cost/kWh } \\
\text { - Small self-discharge/day }\end{array}$ & $\begin{array}{l}\text { - Low energy density } \\
\text { - Suitable site requirements }\end{array}$ \\
\hline & CAES & $\begin{array}{l}\text { - Low capital cost/kWh } \\
\text { - Small Self-discharge/day } \\
\text { - Long lifetime }\end{array}$ & $\begin{array}{l}\text { - Suitable site requirements } \\
\text { - Fuel requirement }\end{array}$ \\
\hline \multirow[t]{2}{*}{$\begin{array}{l}\text { Electrical } \\
\text { storage } \\
\text { systems }\end{array}$} & SES & $\begin{array}{l}\text { - High power density } \\
\text { - Long cycle life } \\
\text { - Small environmental impact }\end{array}$ & $\begin{array}{l}\text { - Low energy density } \\
\text { - High self-discharge/day }\end{array}$ \\
\hline & SMES & $\begin{array}{l}\text { - High power density } \\
\text { - Fast response } \\
\text { - Long lifetime } \\
\text { - High efficiency }\end{array}$ & $\begin{array}{l}\text { - Low energy density } \\
\text { - Temperature sensitivity } \\
\text { - High capital cost/kWh } \\
\text { - High self-discharge/day }\end{array}$ \\
\hline \multirow{4}{*}{$\begin{array}{l}\text { Chemical } \\
\text { Storage } \\
\text { systems }\end{array}$} & LAB & $\begin{array}{l}\text { - Mature technology } \\
\text { - Low capital cost/kWh }\end{array}$ & $\begin{array}{l}\text { - Short lifetime } \\
\text { - Temperature sensitivity }\end{array}$ \\
\hline & NCES & $\begin{array}{l}\text { - High power density } \\
\text { - Longer lifetime and less } \\
\text { temperature sensitivity compared to } \\
\text { LAB }\end{array}$ & $\begin{array}{l}\text { - Memory effect } \\
\text { - High self-discharge/day }\end{array}$ \\
\hline & LIES & $\begin{array}{l}\text { - High efficiency } \\
\text { - High power density } \\
\text { - Lighter and smaller than NCES }\end{array}$ & $\begin{array}{l}\text { - High capital cost } / \mathrm{kWh} \\
\text { - Special charging requirements } \\
\text { to keep voltage and current } \\
\text { within safe limits }\end{array}$ \\
\hline & NaSES & - High power \& energy densities & - High capital cost/kWh \\
\hline
\end{tabular}


- Design safety concerns

- High self-discharge/day

- High temperature requirement $\left(320-340^{\circ} \mathrm{C}\right)$

ZEBRA - Overcharge and discharge Capability.

- Safer than NaSES

FBES - Decoupled power and energy capacities

- High power density

- Full discharge capability

-Small self-discharge/day

HES - Higher capacity than batteries

- Negligible self-discharge/day
- Lower energy and power densities compared to NaSES

- More expensive than NaSES

- High self-discharge/day

- High temperature requirement $\left(250-350^{\circ} \mathrm{C}\right)$

- Low energy density

- Difficult maintenance

- Complicated design requiring moving parts such as pumps

- Immature technology

- Low efficiency

For a standalone WT, installed in a windy location, the storage device is mainly used for short term power balancing. Such an application requires certain characteristics of the storage device used. Table 1.3 lists the requirements of energy storage system (ESS) to be employed in small-scale standalone WECS, supplying a remote area.

In general, PHES and CAES have reduced reliability and slow transient response due to moving parts. Moreover, they require large areas for installation. Therefore, they are not good options for small-scale standalone WECS. On the other hand, they are very viable for energy management in large power (i.e., hundreds of Mega-Watts) applications [98].

On the other hand, batteries and super-capacitors are reliable and fast in response due to the absence of kinetic components. Among the batteries, Lead Acid Batteries (LABs) feature a well-established energy storage technology and thus still represent a low-cost option for standalone WECS [99]. However, they suffer short cycle life. One option for overcoming this drawback and improving overall performance is integrating LABs with SESs [100]. 
Table 1.3: Energy storage requirements for a small-scale standalone WECS.

\begin{tabular}{|c|c|}
\hline Requirement & Comments and justification \\
\hline High reliability & $\begin{array}{l}\text { Wind power is intermittent and energy storage device } \\
\text { must be available to compensate for power mismatch. }\end{array}$ \\
\hline Fast response & $\begin{array}{l}\text { Storage device should rapidly respond to charge/discharge } \\
\text { commands because wind and load are stochastically } \\
\text { fluctuating. }\end{array}$ \\
\hline Short ramping time & $\begin{array}{l}\text { Storage device should be fast-response and able to ramp } \\
\text { up and down from no power to rated power and vice versa } \\
\text { within a reasonable time. }\end{array}$ \\
\hline High power/energy capability & $\begin{array}{l}\text { Short-term power balance requires a storage device with } \\
\text { high power density, whilst energy management } \\
\text { applications require devices with high energy density. }\end{array}$ \\
\hline High efficiency & $\begin{array}{l}\text { Efficiency of storage device is important, especially when } \\
\text { delivering the whole power demand during turbine's } \\
\text { shutdown. }\end{array}$ \\
\hline Low maintenance requirements & Maintenance tends to be very costly in remote areas. \\
\hline \multicolumn{2}{|c|}{$\begin{array}{l}\text { Based on the above discussions, the advantages and drawbacks of storage devices provided } \\
\text { Table 1.2, and the requirements given in Table 1.3, Table } 1.4 \text { assesses the feasibility of each } \\
\text { chnology for a small-scale standalone WECS supplying a remote community. According to } \\
\text { e table, LAB and SES seem to have the highest feasibility for low power off-grid } \\
\text { plications. An excellent performance can be obtained by combining the two technologies in } \\
\text { hybrid storage system. However, in order to reduce the initial cost, LAB is selected for } \\
\text { orage purposes in this research, as a well-established storage technology, offering satisfactory } \\
\text { erformance. If cost is not an issue, Lead acid batteries can be replaced by Lithium-Ion } \\
\text { atteries featuring longer cycle life and lighter weight. }\end{array}$} \\
\hline
\end{tabular}


Table 1.4: Feasibility of Energy Storage Technologies for small-scale standalone WECS.

\begin{tabular}{|c|c|c|}
\hline Technology & Feasibility level & Comments and justification \\
\hline FES & Medium & -Reduced reliability due to moving parts \\
\hline PHES & Very Low & $\begin{array}{l}\text { - Low reliability due to moving parts } \\
\text { - Long response time } \\
\text { - Site requirements may not be met }\end{array}$ \\
\hline CAES & Very Low & $\begin{array}{l}\text { - Gas requirement makes it costly } \\
\text { - Low reliability due to moving parts } \\
\text { - Site requirements may not be met } \\
\text { - Long response time }\end{array}$ \\
\hline SES & High & - High efficiency \\
\hline SMES & Medium & $\begin{array}{l}\text { - High production cost } \\
\text { - High temperature sensitivity }\end{array}$ \\
\hline LAB & High & - Well-established and low-cost technology \\
\hline NCES & Medium & $\begin{array}{l}\text { - Undesired memory effect } \\
\text { - High self-discharge ratio/day }\end{array}$ \\
\hline LIES & Medium & $\begin{array}{l}\text { - High production cost } \\
\text { - Special charging requirements }\end{array}$ \\
\hline NaSES & Medium & $\begin{array}{l}\text { - High production cost } \\
\text { - High temperature requirement }\end{array}$ \\
\hline ZEBRA & Medium & $\begin{array}{l}\text { - More expensive than NaSES } \\
\text { - High temperature requirement }\end{array}$ \\
\hline FBES & Low & $\begin{array}{l}\text { - Difficult maintenance } \\
\text { - Complicated design, requiring moving parts }\end{array}$ \\
\hline HES & Medium & - Immature technology \\
\hline
\end{tabular}




\subsection{Research Objectives}

Based on the assessment of the state-of-the-art in wind-turbine generators, power electronic converters, and energy storage technologies, the focus in this work will be on developing a low-power standalone WECS based on current-source inverter.

The main objectives of this research are:

- Selecting the most promising generator type based on a thorough comparative evaluation.

- Selecting an appropriate CSC-based configuration for standalone WECS.

- Introducing a low-cost small-scale WECS featuring a CSI and a novel integration system for Lead Acid battery-based energy storage system.

- Developing overall dynamic mathematical model for the proposed system.

- Investigating the stability, controllability and observability of the proposed system.

- Designing closed-loop controllers to take care of the following tasks:

1. Maximum power point tracking (MPPT) on the generator side;

2. DC-link current control;

3. Load-side voltage magnitude and frequency control under both balanced and unbalanced three-phase loads; and

4. Power management among generator, load, dump load and storage battery.

- Proposing a dump load-less version of the proposed system.

\subsection{Thesis Layout}

The rest of this thesis is organized as follows:

- The most promising generator type for small-scale standalone WECS supplying a remote area is identified in Chapter 2.

- Chapter 3 investigates various current-source converter topologies for wind power generation system. The comparison between these topologies leads to the selection of a simple and low-cost converter configuration, offering satisfactory performance for low-power low voltage WECS. This chapter introduces the structure of the proposed system, with a novel scheme for the integration of a battery-based energy storage. 
- Chapter 4 carries out the derivation of dynamic and steady-state models of the proposed system in the $d q$ reference frame. A generic model suitable for balanced/unbalanced load conditions is proposed in this chapter. Based on the overall model, a linearized model is developed to investigate local stability and system performance.

- The design of a closed-loop control system is discussed in Chapter 5. The control system includes the dc-link current control loop, the generator speed control loop, and the load voltage control loop. The performance of the control system is demonstrated by simulation.

- The main contributions and outcomes of the thesis are summarized in Chapter 6, followed by suggestions for future research work. 


\section{Chapter 2}

\section{Selection of Generator Type for Small-Scale WECS}

In this chapter, the most promising generator type for small-scale standalone WECS, amongst those mentioned in chapter 1 (subsection 1.2.2), will be identified.

\subsection{Evaluation of Conventional and Evolving Generator Types for Standalone WECS}

Selection of the right generator type is of key importance to successful capturing of wind energy under different wind speed conditions, especially at low wind speeds, where the low power available has to be processed by a high-efficiency conversion system.

Selection of an electrical generator for standalone turbine has been briefly discussed in [101], where induction and synchronous generators are compared, concluding that the generator for standalone turbine must be a permanent magnet (PM) machine in order to avoid excitation requirement. The paper misses to address other issues that need to be considered in addition to excitation requirements. Reference [62] has reviewed the key technologies of small-scale offgrid wind turbines. However, among all possible machines, the review has focused on PM generators only. PM generators, especially direct-drive PM synchronous generators, are the most commonly used electric machine for small-scale WTs [62] and have been of interest to many researchers as a viable solution for standalone WECS [42],[43],[48],[49]. However, the attraction to direct-drive PMSG has been based on the criteria of high power density and reliability only. On the other hand, indirect-drive SCIG has been recommended by [31]-[35] as a simple, robust, brushless and cost-effective generator for standalone WECS. However, the attractiveness of such a generator may diminish if efficiency is a main concern.

The above discussion points to the fact that a more comprehensive study should be carried out leading to selection of the most appropriate generator type for a standalone WECS under specific conditions. Some principles for generator selection in small off-grid WTs were listed in [62]. However, some important factors such as control requirements and construction complexity were not considered. Furthermore, excitation requirement was not an issue in [62], since the paper has focused on PM generators only. 
Motivated by lack of a comprehensive and convincing approach to selection of the right generator for a standalone wind turbine, a thorough study, considering all possible options, has been conducted in [102]. The study evaluated the nine generator types considered for WECS in the literature review covered in chapter 1 (subsection 1.2.2); i.e., WRIG, DFIG, BDFIG, BDFRG, SCIG, WRSG, PMSG, PMIG, and SRG. The evaluation has been conducted on the basis of efficiency, reliability, cost, operation and maintenance requirements, construction complexity, control complexity, excitation requirements and noise level associated with each generator type.

The main observations made based on the study are summarized below.

- Wound-Rotor Induction Generator (WRIG): Soft starter requirement, limited speed range and reduced efficiency due to the power loss in the external resistance are the major drawbacks of WRIG-based WECS. Moreover, the presence of slip rings and brushes, requiring regular maintenance and replacement, makes WRIG not an attractive option for remote area applications, where maintenance is difficult and costly.

- Doubly-Fed Induction Generator (DFIG): The feature of reduced power converter rating offered by DFIG-based WECS may not be a big attraction in the case of standalone wind turbine systems, where power level is relatively low (ranging from a few kilowatts to a few hundred kilowatts). Moreover, DFIG has the drawback of unavoidable use of brushes and slip rings, reducing its reliability and increasing its maintenance requirements.

- Brushless Doubly-Fed Induction Generator (BDFIG): Although brush and slip ring problems do not exist in BDFIG, giving it an advantage from efficiency point of view, large size and construction complexity are issues that can defeat its attractiveness for small WTs. The same drawbacks are present in Brushless Doubly-Fed Reluctance Generator (BDFRG), despite offering a higher efficiency.

- Squirrel-Cage Induction Generator (SCIG): SCIG is a simple, robust, brushless and cost-effective generator for standalone WECS.

- Wound-Rotor Synchronous Generator (WRSG): The need for an external dc source to excite the rotor winding via brushes and slip rings, or a brushless excitation system featuring higher complexity and cost, is the main obstacle for adopting WRSG option in off-grid applications. 
- Permanent-Magnet Synchronous Generator (PMSG): Thanks to its high-efficiency, PMSG has recently gained widespread acceptance as a viable option in direct-drive, small-scale standalone WECSs.

- Permanent-Magnet Induction Generator (PMIG): Adding an improved power factor and a better performance to the advantages of the SCIG, PMIG has a very good potential to serve as a direct-drive generator in isolated WECS.

- Switched-Reluctance Generator (SRG): SRG has the potential to become a good solution for direct-drive WECS in off-grid applications. Although application of SRG in wind energy systems was proposed in the early 1990s, its performance evaluation has been limited to simulation and some laboratory tests, with no field implementation. Thus, when compared with PMSG- and SCIG-WECS, SRG-WECS is still considered to be in early stages of development.

Based on the above remarks, SCIG, PMSG and PMIG seem to be the most suitable generator types for standalone WECS. However, PMIG is relatively an immature machine, when compared to PMSG and SCIG. Therefore, the advantages and drawbacks of PMSG and SCIG will be compared in more details, leaving PMIG to be visited next.

\subsubsection{SCIG-WECS versus PMSG-WECS}

The comparison in this subsection will be on the basis of topology, efficiency, reliability, control complexity, cogging torque, noise, and cost.

\section{A) Topology}

PMSG -based WECS offers an advantage over SCIG-based WECS in terms of possibility of eliminating the need for gearbox. Thus, they are called gearless-PMSG and geared-SCIG, respectively. Fig. 2.1 and Fig. 2.2 show typical topologies for SCIG- and PMSG-based standalone WECS, respectively. Since PMSG is self-excited, a three-phase diode rectifier can be used as the generator-side converter, as shown in Fig. 2.2(a). In contrast, a VAR compensator, such as a capacitor bank, is required to excite the SCIG if a three-phase diode rectifier is to be used, as in Fig. 2.1(a). In both cases, a chopper (e.g., a DC/DC boost converter) is required to control the speed of the generator shaft in order to achieve MPPT. Alternatively, full generator control can be obtained by using pulse-width modulated voltage source rectifier 
(PWM-VSR), as shown in Fig. 2.1(b) and Fig. 2.2(b). This eliminates the need for selfexcitation capacitors for SCIG, as the required reactive power is supplied by the power electronic converter itself. In all topologies shown in Fig. 2.1 and Fig. 2.2, two-level pulse width modulated voltage-source inverters (PWM-VSI) are used as the load-side converters.

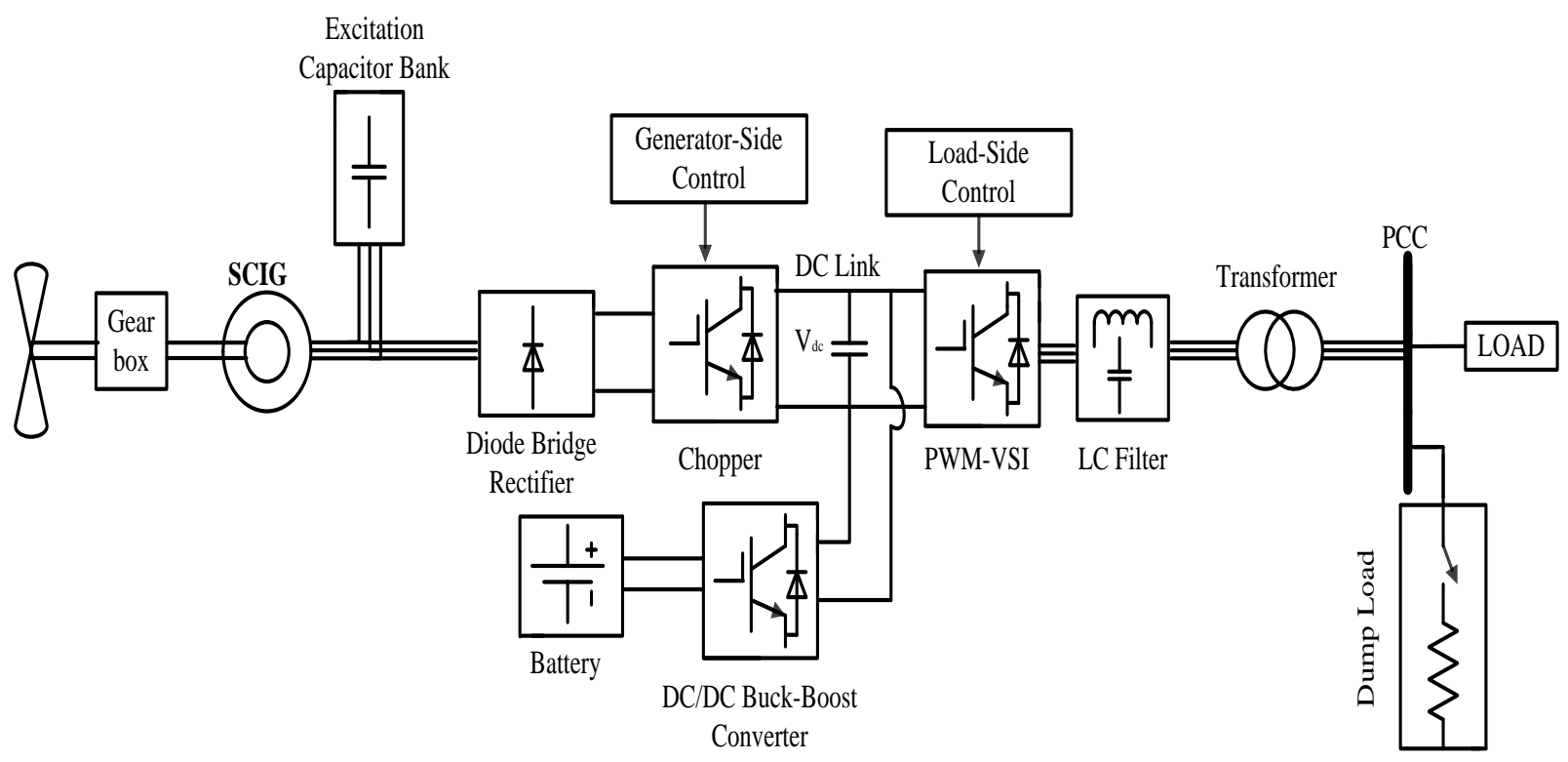

(a)

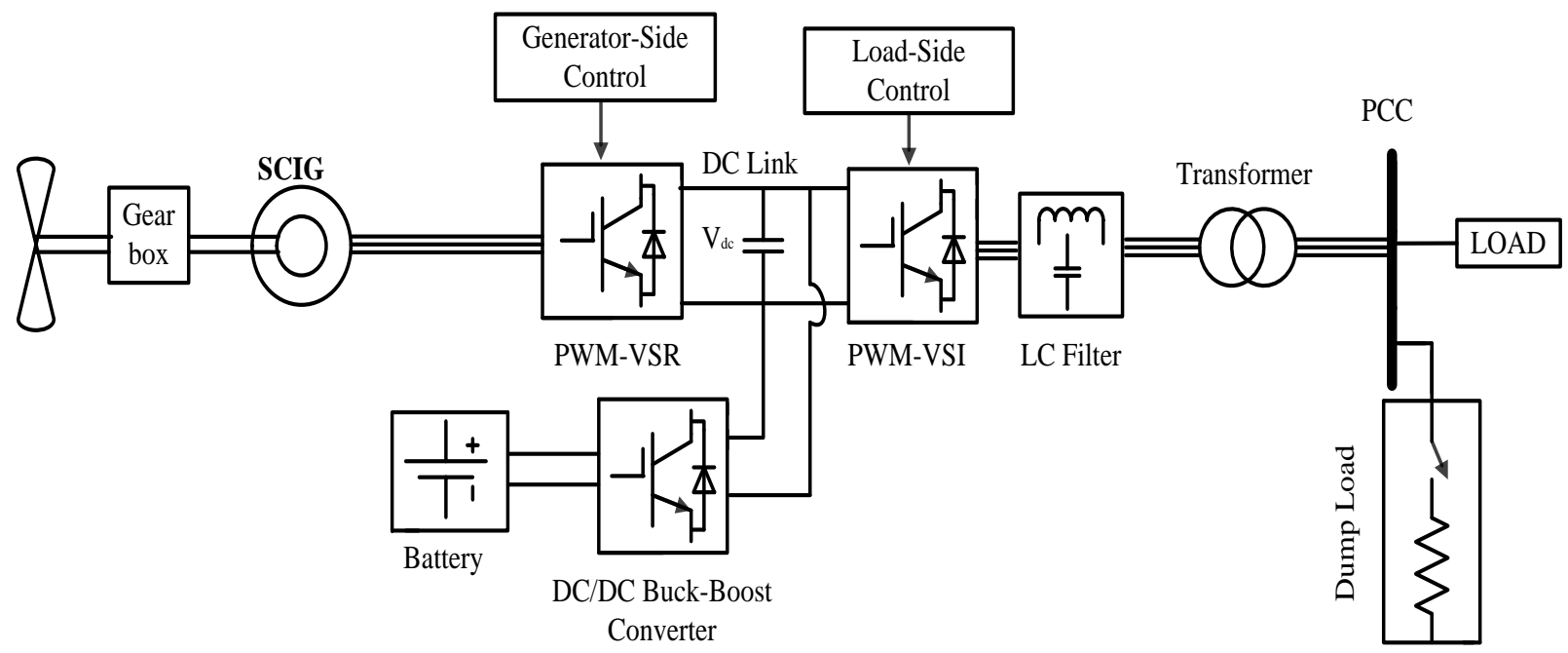

(b)

Fig. 2.1: SCIG-based standalone WECS: (a) with generator-side diode bridge rectifier, and (b) with generator-side voltage-source rectifier. 


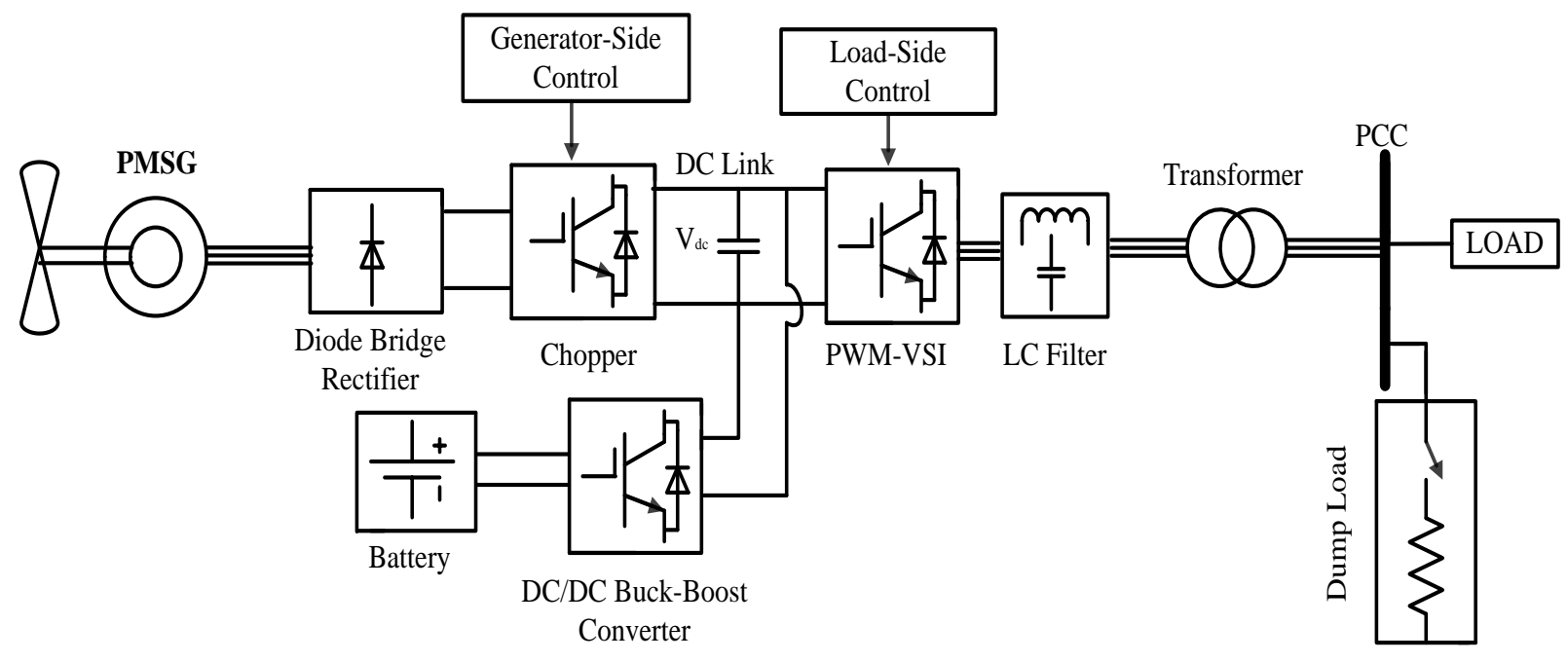

(a)

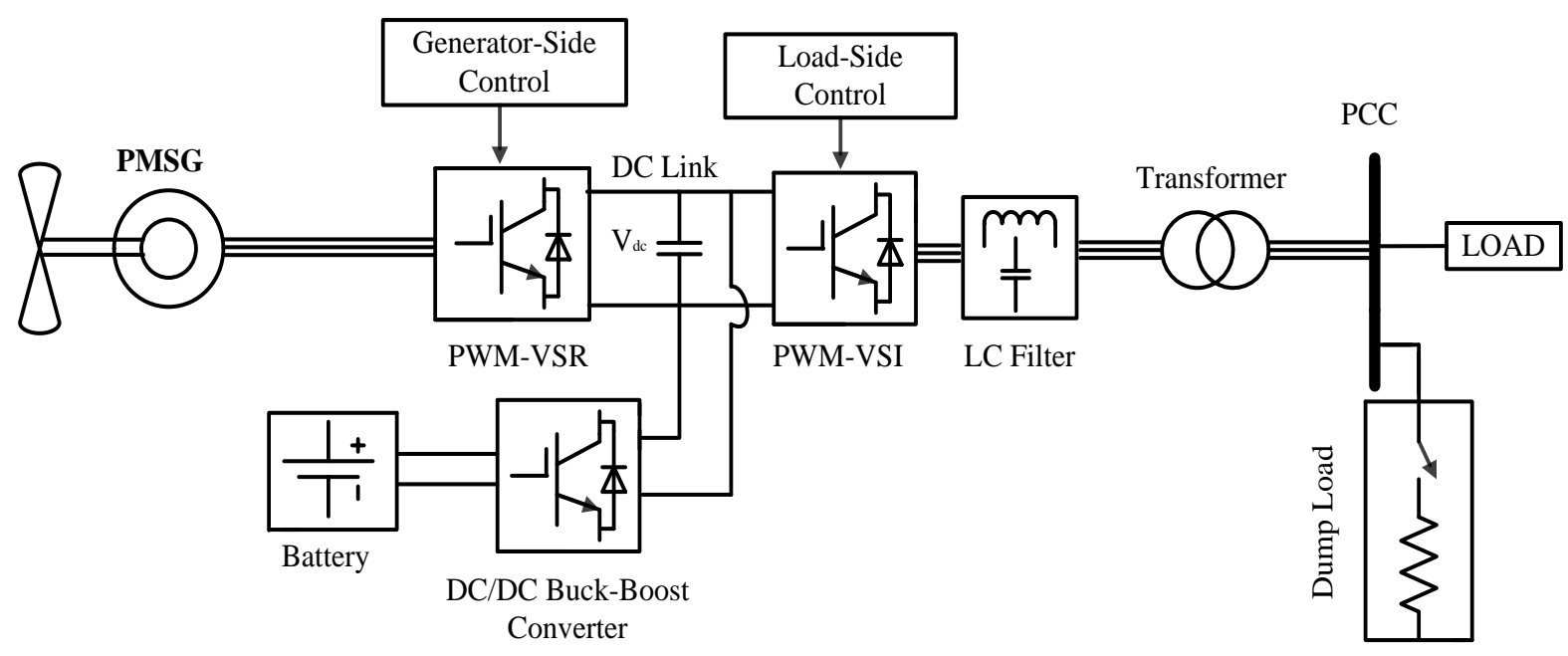

(b)

Fig. 2.2: PMSG-based direct-drive standalone WECS: (a) with generator-side diode bridge rectifier, and (b) with generator-side voltage-source rectifier.

The fact that in the system of Fig. 2.2(a) a diode rectifier can be used without the need for self-excitation capacitors, is considered a big advantage for PMSG-WECS over SCIG-WECS. Indeed, it is a trend to use a diode rectifier and a boost dc/dc converter with PMSG-WECS, as a simple and cost-effective option [62],[63]. 


\section{B) Efficiency}

Due to presence of permanent magnets in PMSG, it is not necessary to supply magnetizing current to the stator for a constant air-gap flux. Therefore, the stator current is only responsible for producing the torque component and hence PMSG, when compared to SCIG, will operate at a higher PF, leading to higher efficiency. SCIG, in contrast, needs to be connected to an external VAR source, in order to establish the magnetic field across the air gap. This results in a low power factor and efficiency. In general, induction generators are less efficient than synchronous generators with comparable ratings [103].

\section{C) Reliability}

Reliability of a wind turbine can be measured by frequency and duration of failures in the system [104]. The gearbox requires regular maintenance and is not immune to failure. If it fails, the repair required is a major task. Studies have shown that the gearbox has a very long downtime per failure when compared with other components of WECS [105]. Thus, the elimination of gearbox in direct-drive PMSG-based WECS can significantly improve the reliability of the system. However, direct-drive systems feature higher number of failures in generator and power electronic converters [106] due to direct transfer of wind turbine rotor torque fluctuations to the generation side; however, the downtime of direct-drive systems due to power electronics or generator failures is definitely much shorter than those of gearbox in indirect drive systems.

Although gearless design is an advantage for PMSG-based WECS over SCIG-based WECS, the fact that the reliability of PMSG can be affected by permanent magnet's demagnetization and change of characteristics under harsh environmental conditions (such as high temperatures), is considered a serious disadvantage.

As far as the generator type is concerned, real data has shown that synchronous generatorbased turbines suffer higher failure rates than those using induction generators [107].

\section{D) Control Complexity}

In variable-speed WECS, the generator shaft speed is controlled to achieve MPPT, which is of key importance in wind energy systems. 
SCIG is one of the simplest machines in terms of control requirements. Control techniques suitable for SCIG, such as direct field oriented, indirect field oriented and direct torque control, are very well-known and well-established. In contrast, one of the drawbacks of PMSG is its control complexity, which is caused by the fact that the magnet excitation cannot be varied and hence the output voltage of PMSG will vary with load. This problem can be solved by capacitive VAR compensation or an electronic voltage controller, adding to the control complexity. Zero d-axis current, maximum torque per ampere and unity power factor, are three common methods of PMSG control [15].

\section{E) Cogging Torque and Noise}

In PMSG, the interaction between the magnets of the rotor and the slots of the stator generates an undesirable torque, called cogging torque, which causes fluctuations in torque and speed of the shaft. Cogging torque results in vibration and noise in the machine, especially at low speed and hence it can negatively affect the cut-in speed of the PMSG turbine [108]. Unlike PM synchronous machines, the phenomenon of cogging torque is not significant in induction machines [109]. However, a geared-SCIG-based wind turbine has another source of noise as a result of presence of gearbox in the drive train [110]. In summary, both gearlessPMSG and geared-SCIG WECS have a source of noise, which is not so important if the turbine is installed far away from the community. However, the cogging torque of PMSG does always matter, as it affects the cut-in speed and hence the total $\mathrm{kWh}$ production of the wind turbine, leading to a lower capacity factor. Nevertheless, cut-in speed for SCIG-based wind turbine is also restricted by the generator threshold speed, below which the machine excitation is not

possible. Thus, capacity factor is negatively affected by limitation of cut-in speed in both PMSG and SCIG wind turbines.

\section{F) Cost}

Compared to the geared-SCIG system, the gearless-PMSG system saves on the cost of gearbox. However, the multi-pole structure adds to the cost of gearless-drive PMSG system. Moreover, PM generators are generally more expensive than induction generators due to the high price of magnets. 
The combination of a diode rectifier and a dc/dc converter, shown in Fig. 1.7, is less expensive than a switch-mode voltage-sourced rectifier, shown in Fig. 1.8. The former configuration is commonly used in small-scale, standalone PMSG systems [62]. If the same configuration is to be used with SCIG, there will be an extra cost due to the need for external exciter. However, capital cost comparison should be conducted, considering all system components.

For cost comparison purposes, a $30 \mathrm{~kW}$ wind turbine is selected as an example for small wind turbines in off-grid applications. Such a turbine can supply power to a small village, a large farm or a small enterprise, when equipped with an energy storage system. Table 2.1 shows the prices for a gearless-drive PMSG-WECS and a geared-drive SCIG-WECS with similar power ratings (i.e., 30kW) [111]-[113]. The comparison reveals the cost advantage of geared-SCIG turbine with respect to gearless-PMSG turbine. The combined cost of SCIG and gearbox is around $50 \%$ of PMSG cost. Although the price difference depends on power rating and varies from one manufacture to another, and from one country to another, the price ratio between geared SCIG and gearless PMSG systems are currently significant due to the involvement of PM materials in the latter system.

Operation and maintenance (O\&M) cost is another contributor to a WECS overall cost. O\&M cost includes costs of regular inspection, repair, spare parts and insurance [114]. When comparing geared-SCIG and gearless-PMSG systems, the O\&M is mainly associated with gearbox and generator. The O\&M cost for geared-SCIG is expected to be relatively high due to the presence of gearbox, which requires regular maintenance and expensive spare parts if a repair is needed [105]. On the other hand, the gearless-PMSG's O\&M cost is due to high rate of failures in generator and power electronic converters [106], but it is still much lower than the gearbox maintenance cost. Insurance of a wind turbine is also counted as a part of O\&M expenses. The insurance of a geared-SCIG turbine is considerably affected by the gearbox. The cost of replacing a gearbox can reach $10 \%$ of the original construction cost of the wind turbine [115], which defeats the advantage of low capital cost in a geared-SCIG wind turbine. On the other hand, the insurance cost is generally proportional to capital cost and hence a gearless-PMSG turbine's insurance is negatively affected by its high capital cost, which is expected to increase further in future due to unreliable supply of permanent magnet material in the global market. In summary, although the presence of gearbox in a geared-SCIG turbine 
adds to the O\&M expenses, its overall cost, including capital cost, is still lower than that of a gearless-PMSG wind turbine.

Table 2.1: Cost Comparison of 30kW PMSG- and SCIG-Based WECSs.

\begin{tabular}{lcc}
\hline Component & PMSG-WECS [111] & SCIG-WECS [112] \\
& US\$ & US\$ \\
\hline Blades (3- Horizontal axis) & 3,890 & 2,120 \\
Gearbox & None & 4,838 \\
Generator & 13,400 & 1,400 \\
Controller & 8,500 & 8,630 \\
$\quad$ including rectifier, & & \\
dump load and inverter) & & \\
Lead Acid Batteries (144 kWh) [113] & 8,400 & 8,400 \\
\hline Total & $\mathbf{3 4 , 1 9 0}$ & $\mathbf{2 5 , 3 8 8}$ \\
\hline
\end{tabular}

Based on the comparison from the viewpoints of efficiency, reliability (particularly the length of gearbox downtime), and external excitation requirements, the direct-drive PMSG system represents the preferred topology for small-scale, standalone WECS. On the other hand, based on the comparison from the viewpoints of reliability (particularly the failure rate of generator and power converters), machine size and weight, control simplicity, and overall cost, the indirect-drive SCIG system wins against the direct-drive PMSG system. Moreover, PMSG might face a real problem in future due to shortage and monopoly of permanent magnet supply. The resources of permanent magnets, especially the Neodymium type, are almost entirely limited to China. This fact is raising concerns about shortage of PM supply in the near future as a result of considerable increase in demand that is expected due to proliferation of Hybrid Electric Vehicles and Electric Vehicles that commonly use PM synchronous machines for their traction motors [116].

The main advantages and drawbacks of the geared SCIG- and gearless PMSG-based wind energy conversion systems are summarized in Table 2.2. 
Table 2.2: SCIG-WECS versus PMSG-WECS.

\begin{tabular}{|c|c|c|}
\hline Topology & Indirect-drive SCIG & Direct-drive PMSG \\
\hline Common properties & \multicolumn{2}{|c|}{$\begin{array}{l}\text { - Brushless machine } \\
\text { - No windings in rotor } \\
\text { - Full active and reactive power control } \\
\text { - Good control bandwidth }\end{array}$} \\
\hline Advantages & $\begin{array}{l}\text { - Robust operation } \\
\text { - Low cost } \\
\text { - Low generator maintenance } \\
\text { - Ease of control }\end{array}$ & $\begin{array}{l}\text { - Gearless } \\
\text { - Self excited } \\
\text { - High PF operation } \\
\text { - High efficiency } \\
\text { - No rotor copper loss }\end{array}$ \\
\hline Disadvantages & $\begin{array}{l}\text { - Gear box losses and } \\
\text { maintenance } \\
\text { - Need for external excitation } \\
\text { - Low efficiency }\end{array}$ & $\begin{array}{l}\text { - Magnet cost } \\
\text { - PM Demagnetization } \\
\text { - Large size } \\
\text { - Complex control } \\
\text { - Cogging torque }\end{array}$ \\
\hline
\end{tabular}

\subsubsection{PMIG versus SCIG and PMSG for Standalone WECS}

As mentioned above, in spite of its advantageous features, SCIG suffers from low power factor and low efficiency, as the machine requires magnetizing current from a source of reactive power. If part of the magnetic flux is supplied within the machine, the magnetizing current will be reduced and hence the power factor will be improved. This can be achieved by incorporating permanent magnets within a cage-rotor IG. Such a configuration is called permanent-magnet induction generator (PMIG). The stator of the PMIG is similar to that of the conventional IG, but its rotor design is different. PMIG has two rotor parts: a squirrel-cage rotor and a PM rotor. As the squirrel-cage rotor is partially excited from the PM rotor, the reactive power required from an external source is reduced. Moreover, PMIG can be directly driven without a gearbox. In other words, PMIG, to some extent, combines the advantages of SCIG and PMSG. Fig. 2.3 shows the possible configurations of standalone WECS using PMIG. Compared to SCIG-WECS, shown in Fig. 2.1, the gearbox is no longer an essential 
component. Moreover, the size of the capacitor bank in PMIG-WECS shown in Fig. 2.3(a), is considerably smaller than that in SCIG-WECS shown in Fig. 2.1(a).

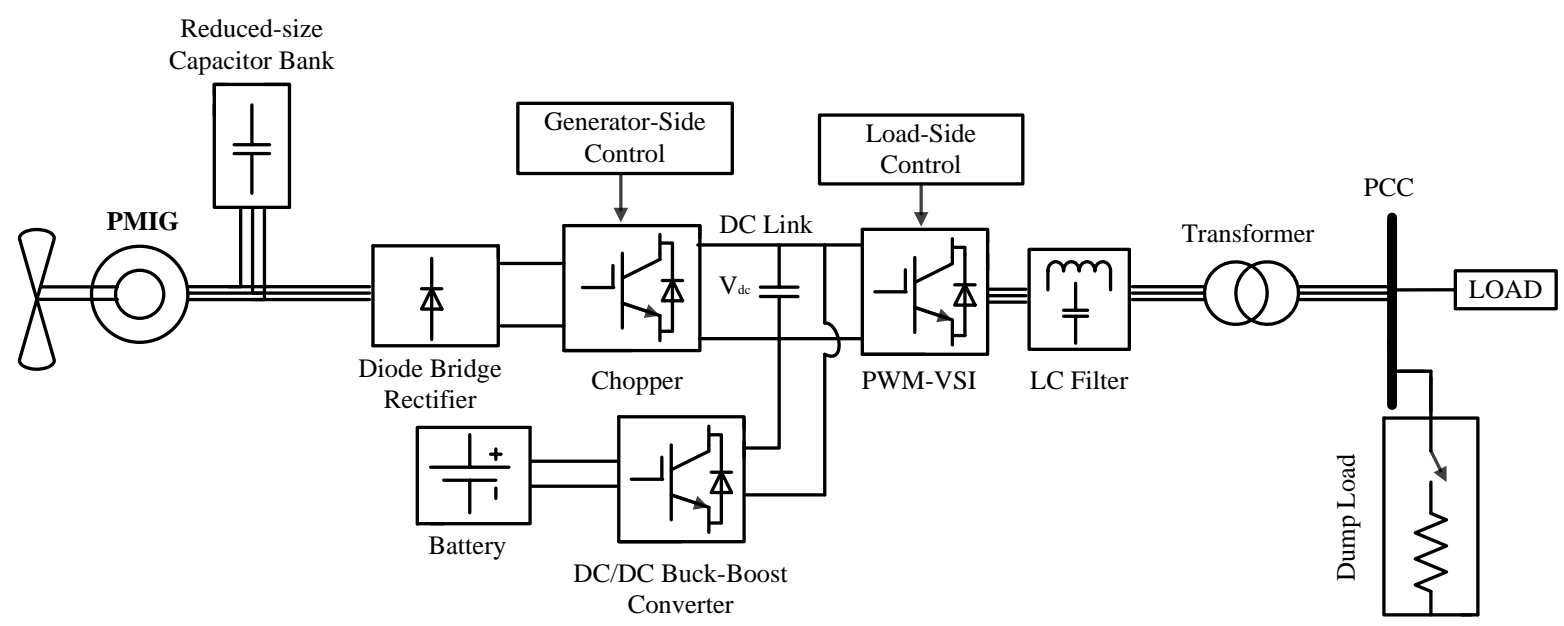

(a)

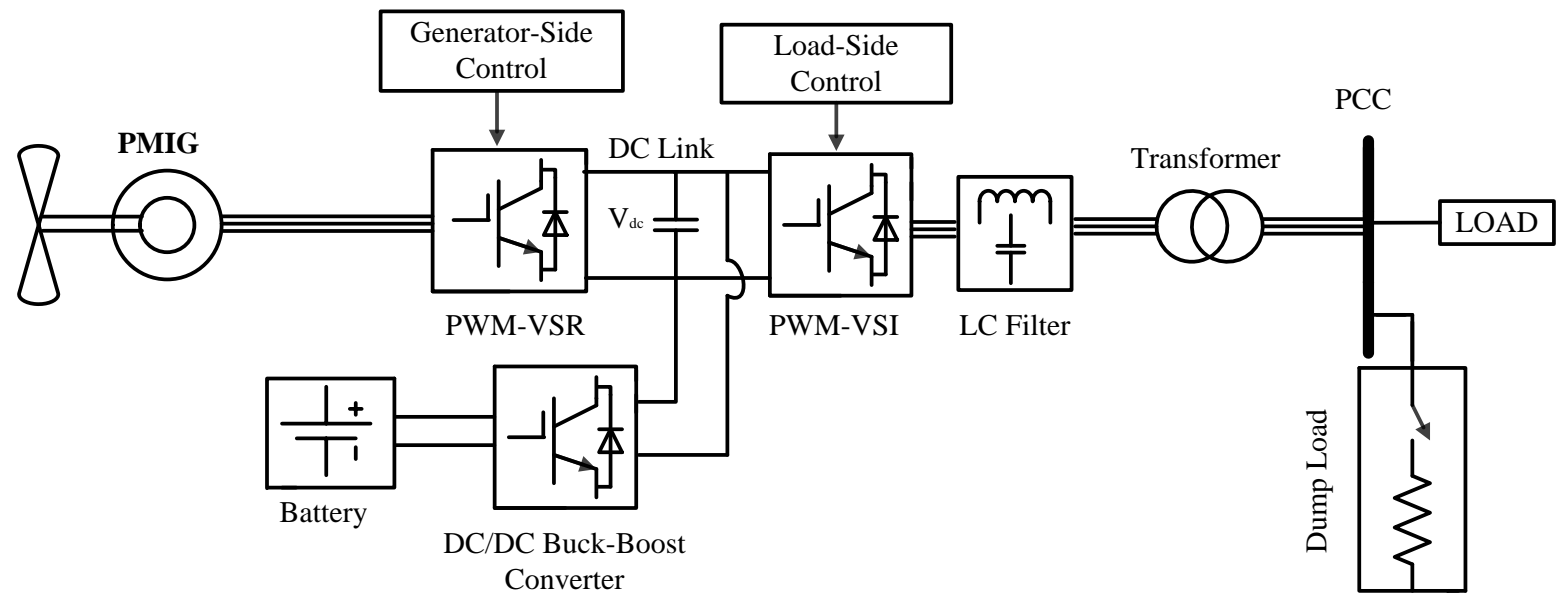

(b)

Fig. 2.3: PMIG-based direct-drive standalone WECS: (a) with generator-side diode bridge rectifier, and (b) with generator-side voltage-source rectifier.

In recent years, some manufacturers [54],[55] have started considering PMIG as a good alternative for PMSG, especially for small-scale wind turbines. However, the construction of PMIG is complex due to its double-rotor design which also increases the effect of cogging torque in the machine. Another drawback of PMIG is the increase in cost due to magnet installation. Table 2.3 shows the cost information for a 30kW PMIG-WECS. Based on the 
information given in Table 2.1, PMIG is slightly less expensive than PMSG, but much more costly than SCIG of the same power rating.

Table 2.3: Cost of 30kW PMIG-Based WECS.

\begin{tabular}{lc}
\hline Component & PMIG-WECS[54] \\
& US\$ \\
\hline Blades ( 3 - Horizontal) & 3,709 \\
Gearbox & None \\
Generator & 12,516 \\
Controller & 8,225 \\
$\quad$ (including rectifier, dump load and inverter) & 8,400 \\
Lead Acid Batteries (144 kWh) [113] & $\mathbf{3 2 , 8 5 0}$ \\
\hline Total &
\end{tabular}

\subsubsection{Indices for Selecting the Preferred Generator}

Based on the discussions in the previous subsections, three wind generator configurations, namely geared-SCIG, gearless-PMSG and gearless-PMIG, were selected among all configurations for comparison purposes. Compared to SCIG and PMSG, PMIG is relatively new to the wind energy market. The focus of the comparison is on the generator and the associated drive train. Therefore, the three configurations are assumed to:

1. have identical rotor blades;

2. have similar generator-side rectifiers (i.e., a three-phase diode bridge rectifier in addition to a dc/dc converter);

3. have similar three-phase inverters;

4. have similar types and ratings of energy storage units;

5. be subjected to the same environmental conditions;

6. have comparable $\mathrm{kW}$ ratings;

7. be designed for off-grid application over their entire life time; and

8. be land-based wind turbines. 
According to the discussions made in the previous subsections, Table 2.4 compares the three recommended systems in terms of different indices. The indices are set up in order, starting with the most important index for a small-scale, off-grid WECS supplying a remote community, where failure in the supply system is a critical issue. Therefore, the top priority is given to the reliability of the system, followed by its continuous O\&M cost, while the lowest priority is given to construction complexity, that is reflected in topology's size and weight (increasing transportation and installation costs), and noise level, assuming that the turbine is not very close to the community that is supplied. Due to difficulties in giving an accurate quantitative analysis (i.e., in terms of numbers or percentages), a qualitative comparison is performed based on the discussions conducted in the previous subsections. For each index, each system is assigned a number $(1,2$ or 3$)$ to show its rank for that index with respect to the other two systems. If two systems are assigned the same number for a specific index, they are at the same level for that index. As shown in the table, geared-SCIG system is prominent in $58.3 \%$ of the indices whilst gearless-PMSG system dominates in $41.7 \%$ of the indices. Also, gearless-PMIG is similar to the gearless-PMSG in $60 \%$ of its advantages. Therefore, gearedSCIG system prevails in terms of number of indices. However, gearless-PMSG dominates in three of the top-priority indices, namely duration of failure, gearbox O\&M cost and generation efficiency. Nevertheless, geared-SCIG is also dominant in three of the top priority indices, namely frequency of failure, generator O\&M cost, and capital cost. In order to achieve more accurate results, the weight of an index, according to its order, should be included in the comparison. Considering the order of each index $(i)$ and rank of each generator $(R)$ provided in Table 2.4, the credit of each generator $(C)$ is obtained from (2.1).

$$
C=\frac{1}{\sum_{i=1}^{12}\left[(i)\left(R_{i}\right)\right]}
$$

It has been found that SCIG scores the highest credit, while the lowest credit is gained by PMIG. Taking SCIG as base, the relative credit of PMSG and PMIG are $89 \%$ and $74 \%$, respectively. Therefore, the geared-SCIG proves to be the most suitable for small-scale offgrid WECS, provided that its reliability and efficiency can be improved, while maintaining the advantage of lowest overall cost. 
Table 2.4: Comparison of the geared-drive SCIG, gearless-drive PMSG and gearless-drive PMIG-WECS configurations.

\begin{tabular}{|c|c|c|c|c|c|c|c|}
\hline $\begin{array}{c}\text { Order } \\
\text { of } \\
\text { index }\end{array}$ & $\begin{array}{l}\text { Index } \\
\text { name }\end{array}$ & $\begin{array}{l}\text { Details of } \\
\text { index }\end{array}$ & $\begin{array}{l}\text { Geared } \\
\text {-SCIG }\end{array}$ & $\begin{array}{l}\text { Gearless } \\
\text {-PMSG }\end{array}$ & $\begin{array}{l}\text { Gearless } \\
\text {-PMIG }\end{array}$ & $\begin{array}{c}\text { Best } \\
\text { option }\end{array}$ & Comments and justifications \\
\hline 1 & \multirow[t]{2}{*}{ Reliability } & $\begin{array}{l}\text { Duration of } \\
\text { failure }\end{array}$ & 2 & 1 & 1 & $\begin{array}{l}\text { PMSG } \\
\& \\
\text { PMIG }\end{array}$ & $\begin{array}{l}\text { Geared-SCIG suffers a very } \\
\text { long downtime per gearbox } \\
\text { failure }\end{array}$ \\
\hline 2 & & $\begin{array}{l}\text { Frequency of } \\
\text { failure }\end{array}$ & 1 & 2 & 2 & SCIG & $\begin{array}{l}\text { Direct-drive WT suffers } \\
\text { higher failure rate }\end{array}$ \\
\hline 3 & \multirow[t]{2}{*}{ O\&M Cost } & Gearbox & 2 & 1 & 1 & $\begin{array}{l}\text { PMSG } \\
\& \\
\text { PMIG }\end{array}$ & $\begin{array}{l}\text { No gearbox in direct-drive } \\
\text { WT }\end{array}$ \\
\hline 4 & & Generator & 1 & 2 & 2 & SCIG & $\begin{array}{l}\text { Generator failures are costly } \\
\text { in direct-drive WTs. }\end{array}$ \\
\hline 5 & Capital Cost & $\begin{array}{l}\text { Cost of } \\
\text { generator and } \\
\text { gearbox }\end{array}$ & 1 & 3 & 2 & SCIG & $\begin{array}{l}\text { PM machines are expensive } \\
\text { due to magnets. }\end{array}$ \\
\hline 6 & Efficiency & $\begin{array}{l}\text { Accounts for } \\
\text { gearbox and } \\
\text { Generator loss }\end{array}$ & 3 & 1 & 2 & PMSG & $\begin{array}{l}\text { Gearless PMSG has neither } \\
\text { gearbox nor rotor copper } \\
\text { losses. It also operates at high } \\
\text { PF. }\end{array}$ \\
\hline 7 & $\begin{array}{l}\text { Excitation } \\
\text { requirements }\end{array}$ & $\begin{array}{l}\text { Reactive power } \\
\text { source }\end{array}$ & 3 & 1 & 2 & PMSG & $\begin{array}{l}\text { SCIG is fully externally } \\
\text { excited. } \\
\text { PMIG is partially externally } \\
\text { excited. } \\
\text { PMSG is fully internally } \\
\text { excited. }\end{array}$ \\
\hline 8 & Magnet problems & $\begin{array}{l}\text { Demagnetiza- } \\
\text { tion \& security }\end{array}$ & 1 & 2 & 2 & SCIG & SCIG has no magnets. \\
\hline 9 & Control simplicity & & 1 & 2 & 3 & SCIG & $\begin{array}{l}\text { SCIG is simple in control } \\
\text { while fixed magnet excitation } \\
\text { in PM machines complicates } \\
\text { their controls. }\end{array}$ \\
\hline 10 & $\begin{array}{l}\text { Construction } \\
\text { simplicity }\end{array}$ & $\begin{array}{l}\text { Number of } \\
\text { poles, diameter } \\
\text { size, and rotor } \\
\text { design }\end{array}$ & 1 & 2 & 3 & SCIG & $\begin{array}{l}\text { Direct-drive PMSG is large } \\
\text { and heavy due to multiple- } \\
\text { pole construction. } \\
\text { PMIG is complicated due to } \\
\text { double rotor design. }\end{array}$ \\
\hline 11 & \multirow{2}{*}{ Noise level } & Drive train & 2 & 1 & 1 & $\begin{array}{l}\text { PMSG } \\
\& \\
\text { PMIG }\end{array}$ & $\begin{array}{l}\text { PMSG \& PMIG have no } \\
\text { gearbox noise. }\end{array}$ \\
\hline 12 & & Generator & 1 & 2 & 2 & SCIG & $\begin{array}{l}\text { SCIG has no significant } \\
\text { cogging torque. }\end{array}$ \\
\hline
\end{tabular}

ORDER OF INDEX (1 TO 12) DENOTES DEGREE OF SIGNIFICANCE/PRIORITY (1: HIGHEST PRIORITY). RANK OF SYSTEM FOR AN INDEX (1, 2 OR 3) DENOTES SUPERIORITY (1: THE BEST OPTION). 


\subsection{Summary}

This chapter gave an analytical evaluation of nine generator types available in wind market and reported in the literature, for small-scale standalone wind turbine applications.

Gearless-drive PMSG-based and geared-drive SCIG-based systems were concluded to be the most desirable solutions among different configurations considered. These two preferred generator types were compared with each other. Construction, efficiency, reliability, control complexity, cogging torque and cost including capital cost as well as operation and maintenance cost of the topology were the criteria for comparison. In terms of efficiency and reliability, the direct-drive PMSG system was found to be the best option. In particular, the direct-drive PMSG with diode rectifier was found to be currently the most preferred topology for small-scale, standalone WECS, as it is less expensive compared to direct-drive PMSG with back to back converter. However, in terms of construction, cogging torque, control simplicity and overall cost, geared-SCIG WECS prevails.

The candidacy of PMIG to replace PMSG in a direct-drive wind turbine was discussed. As an induction machine with improved performance, PMIG has a very good potential to be another alternative for PMSG in small-scale WECS. However, similar to PMSG, PMIG is suffering from issues regarding magnet cost, PM demagnetization, and insecurity of future PM supply.

Finally, the three generation systems, namely geared SCIG, gearless PMSG and gearless PMIG systems were compared with one another, as they are suggested by the discussion to be the top candidates in today's market. A group of indices were used as basis for a qualitative comparison. The system based on geared-SCIG was shown to be the most appropriate scheme for a small-scale standalone WECS, supplying a remote area.

The material of this chapter has appeared in the published journal paper [102]. 


\section{Chapter 3}

\section{Proposed Wind Energy Conversion System}

Based on the objectives stated in Chapter 1 and evaluation of wind generators conducted in Chapter 2, a wind energy conversion system composed of a geared-drive squirrel-cage induction generator and a current-source converter, integrated with a Lead Acid battery storage unit, is proposed in this chapter. The block diagram of the proposed standalone WECS is shown in Fig. 3.1 .In order to assess the feasibility of the proposed system, a number of possible CSCbased configurations that have been proposed for grid-connected WECS will be investigated in this chapter.

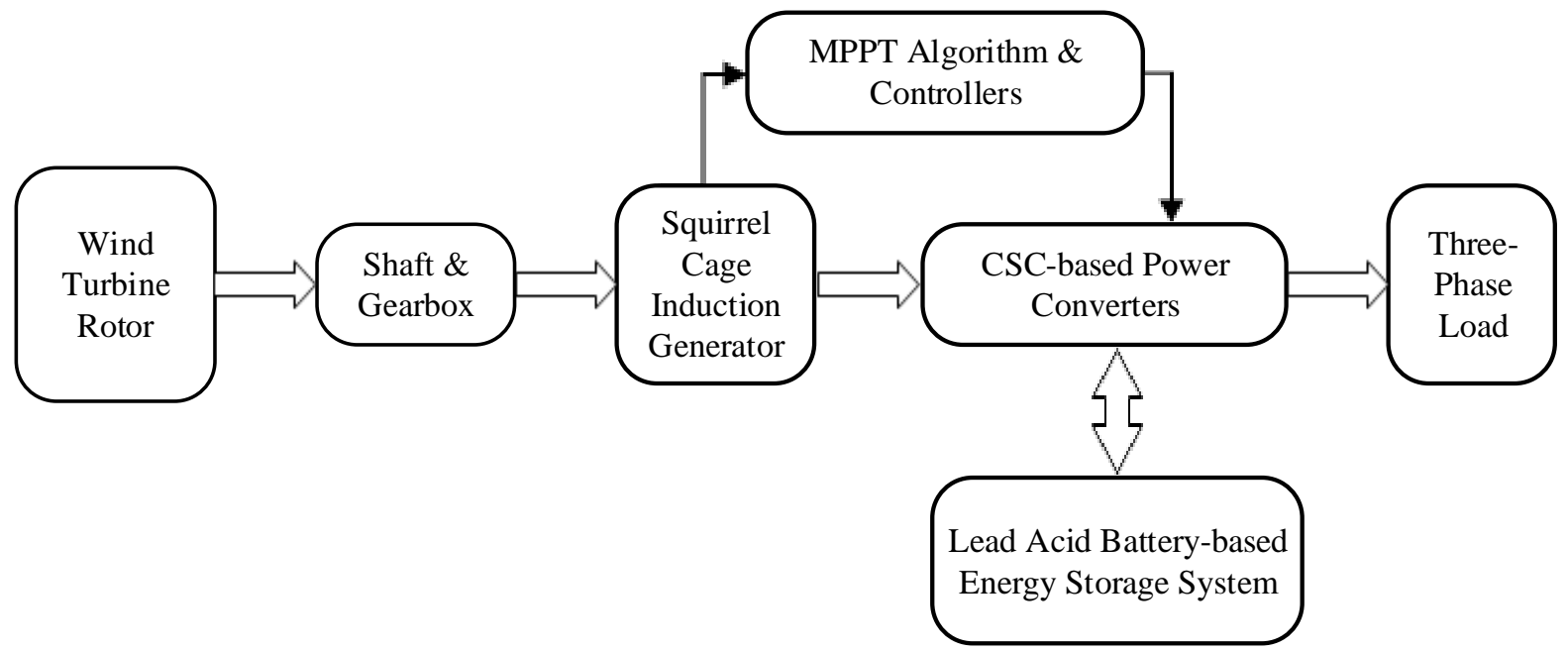

Fig. 3.1: Block diagram of the proposed standalone WECS.

\subsection{Configurations of CSC for Standalone WECS}

In the following subsections, three CSC-based configurations, reported in the literature [73],[74] for grid-connected WECS, will be investigated for standalone WECS. For simulation purposes, the systems are built in Matlab/Simulink environment. In all Simulink models, the current-source inverter is controlled by Sinusoidal PWM technique and the loadside voltage is regulated in the load-side-oriented $d q$ synchronous frame; i.e., the reference of $d$-axis and $q$-axis load voltages are $v_{L d, r e f}=1$ pu and $v_{L q, r e f}=0$, respectively. 


\subsubsection{Topology 1: Diode Rectifier - PWM CSI}

Fig. 3.2 shows a geared-self-excited-SCIG-based WECS using a diode rectifier and a PWM CSI. The generator is excited by a three-phase capacitor bank. The variable generated ac voltage is rectified by the three-phase diode bridge rectifier. The dc capacitor filter $\left(C_{d c}\right)$ assists in smoothing the rectifier output voltage. The dc-link reactor $\left(L_{d c}\right)$ acts as a current source for the PWM-CSI. The size of $L_{d c}$ is selected to reduce ripple in the dc-link current $\left(i_{d c}\right)$ to an acceptable level. Typically, $L_{d c}$ is designed to have a size between 0.6 and $1.2 \mathrm{pu}$ [15]. In topology 1 , the rectification is performed through a line-commutated bridge rectifier. Therefore, the switching frequency is the line frequency and hence a bulky $L_{d c}$ is required. The goal of the PWM-CSI is to produce three-phase line currents at a fixed frequency. The output C-filter $\left(C_{i}\right)$ absorbs the switching harmonics produced by the inverter and defines the output voltage required at the load bus. The $C_{i}$ combined with cable/load inductance forms a secondorder LC filter, improving the quality of voltage delivered to the load. The C-filter design depends on the inverter switching frequency, the LC filter resonance frequency, the allowable line current Total Harmonic Distortion (THD), and the load type [15]. Typically, $C_{i}$ is in range of 0.3 to $0.6 \mathrm{pu}$ for a switching frequency of a few hundred hertz [15], assuming that the frequency of the lowest harmonic injected by the PWM-CSI is higher than the resonant frequency $\left(f_{r}=1 / 2 \pi \sqrt{L C}\right)$ of the Load-side LC filter. For switching frequencies on the order of kilo Hertz, $C_{i}$ is considerably reduced. The PWM-CSI, controlled by synchronous $d q$-axis reference frame PI regulators, regulates the output voltage by varying the modulation index $\left(m_{i}\right)$, while the frequency is set at the desired value (i.e., 50 or $60 \mathrm{~Hz}$ ) in open-loop control. It has to be noted that the minimum value of dc-link current $i_{d c}$ is given by (1.4). Since the CSI controls the load-side voltage and frequency, $i_{d c}$ is left without control. Therefore, the minimum $i_{d c}$ cannot be guaranteed and MPPT cannot be achieved in this configuration. 


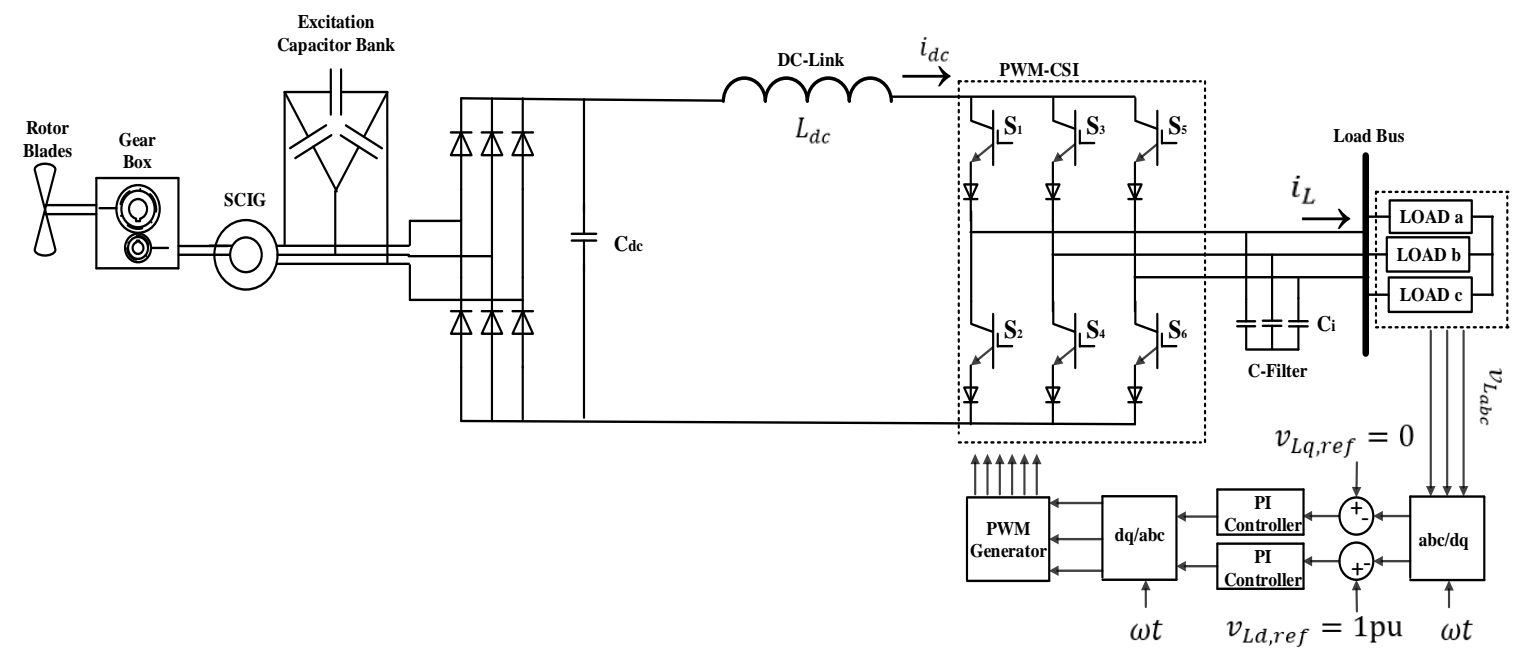

Fig. 3.2:A SCIG-WECS composed of a diode rectifier and a PWM-CSI.

Simulation of this system is carried out on a $30 \mathrm{~kW} / 320 \mathrm{~V}$ standalone WECS feeding a threephase balanced RL load at $380 \mathrm{~V} / 220 \mathrm{~V}$. The load is assumed to have a constant impedance Z, determined from the nominal phase-to-phase voltage (i.e., 1pu), as well as the specified active (P) and reactive $(\mathrm{Q})$ power values. In the process of finding load flow solution, the load impedance is kept constant. The effective $\mathrm{P}$ and $\mathrm{Q}$ are, therefore, varying proportionally to the square of the bus voltage. The system's parameters are given in Appendix A(Table A.1). The rated wind speed is $12 \mathrm{~m} / \mathrm{s}$. The diode-bridge provides no control over the generator torque or speed. The CSI controls the load-side voltage and frequency. Due to the lack of control over the dc-link current or generator, the dc-link current rises uncontrollably, resulting in high reactive power absorbed from the generation side, which prevents generator's flux from building up and causes the generator voltage to collapse in 40 milliseconds, as shown in Fig. 3.3. At this moment, the generator produces zero torque and the shaft over speeds. The minimum $i_{d c}$ is reached at $\mathrm{t}=0.01 \mathrm{~s}$ and $i_{d c}$ keeps increasing until $\mathrm{t}=0.04 \mathrm{~s}$. The stored energy in dc reactor feeds the CSI. However, the load-side voltage collapses once the reactor's stored energy is fully depleted at $\mathrm{t}=0.07 \mathrm{~s}$. 

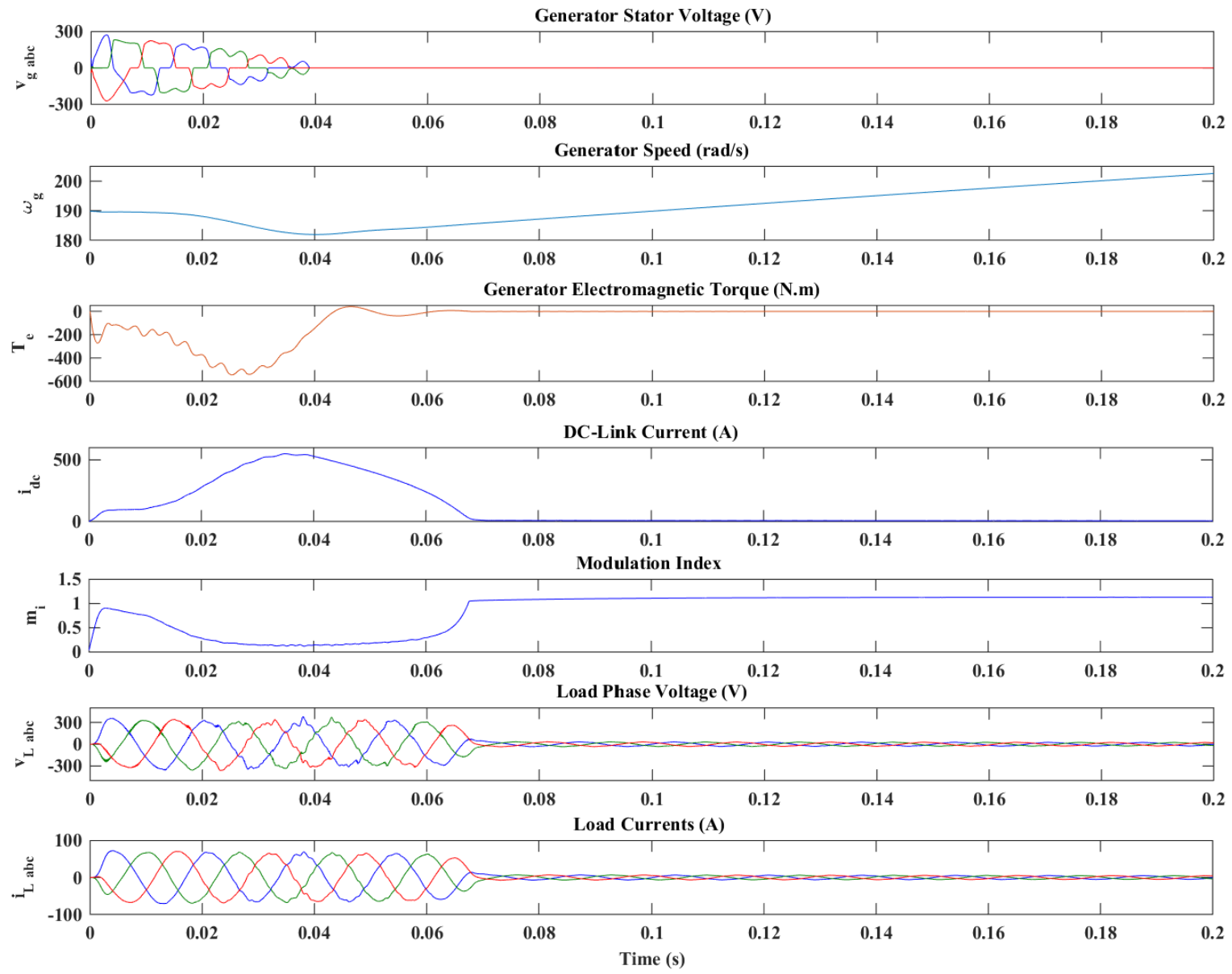

Fig. 3.3: Simulation responses for topology 1.

\subsubsection{Topology 2: Diode Rectifier - Buck Converter - PWM CSI}

In order to control the generator, the system of Fig. 3.2 has to be modified by inserting a dcdc buck converter between the diode rectifier and the CSI, as shown in Fig. 3.4. Compared to topology 1 , a smaller dc-link choke $L_{d c}$ can be used due to the high switching frequency of the buck converter. The buck converter serves as a current booster that provides decoupling between the generation side and the CSI. Hence, the generator-side dc current $\left(i_{d c g}\right)$ is decoupled from the CSI-side dc current $\left(i_{d c}\right)$. Based on optimal value of turbine's tip-speed ratio $\left(\lambda_{\text {opt }}\right)$, the buck converter is used to achieve MPPT by regulating the generator shaft speed $\left(\omega_{g}\right)$ at the corresponding optimum value $\left(\omega_{g, o p t}\right)$ at each wind speed $\left(v_{W}\right)[73]$. In the speed-control loop, $n_{\text {gear }}$ and $r$ denote the gear box ratio and radius of the turbine, respectively. Details of this control loop will be discussed in chapter 5 (subsection 5.4.1). 


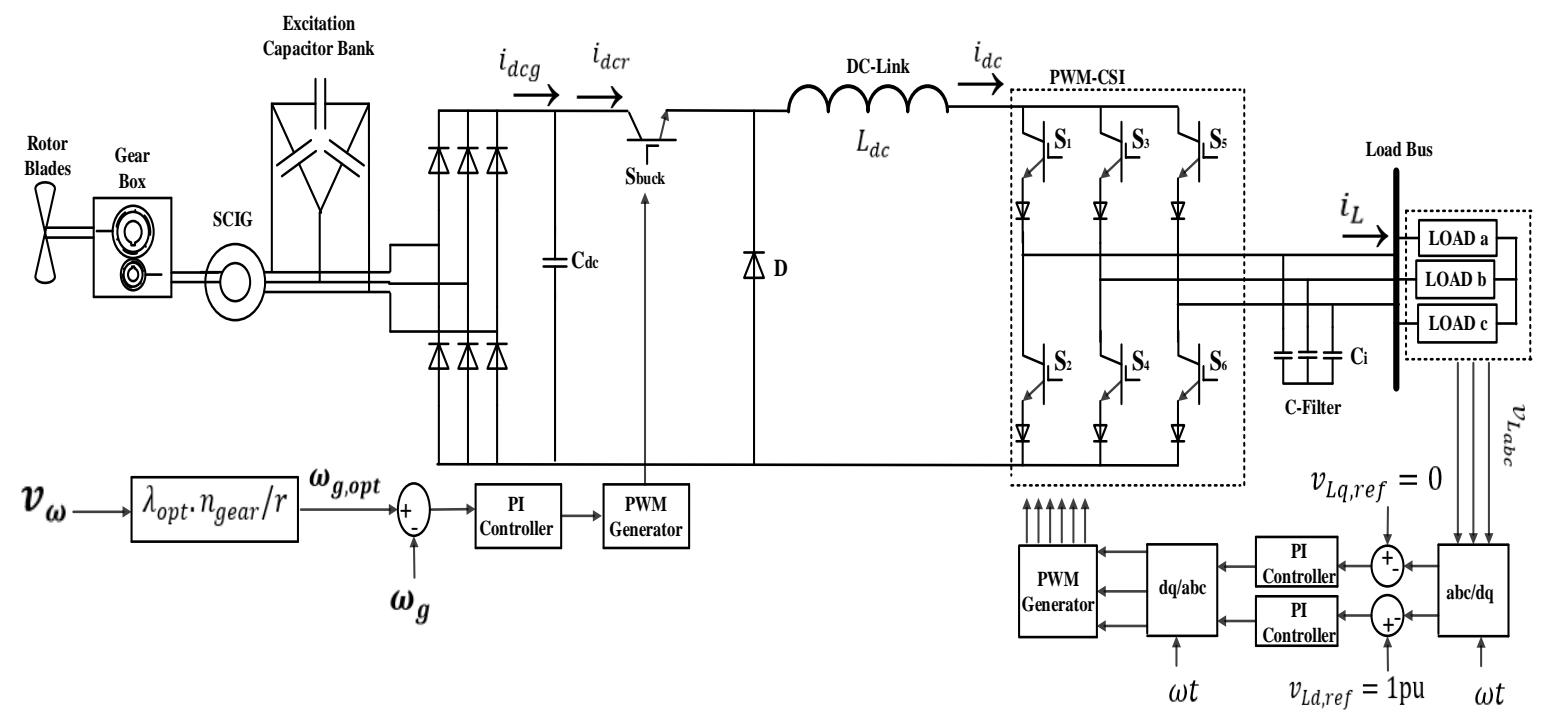

Fig. 3.4: A SCIG-WECS composed of a diode rectifier, a buck converter and a PWM-CSI.

Although the buck converter adds a degree of freedom to the system control, it increases the system's power loss. All the power transferred from generation to load side has to pass through the buck converter, resulting in high conduction losses. In fact, the buck switch needs to withstand a higher stress, compared to those in the CSI. Thus, special care should be practiced in the selection process, from the viewpoints of proper sizing and reliability.

The PWM-CSI regulates the output voltage by varying the modulation index provided that the minimum $i_{d c}$ is guaranteed. However, the inverter-side dc-link current $i_{d c}$ is not controlled in this configuration. Therefore, it may fall below its minimum value under low wind speed and/or heavy load conditions.

To evaluate the performance of this configuration, simulation of a $30 \mathrm{~kW} / 320 \mathrm{~V}$ standalone WECS, feeding a three-phase RL balanced load at 380V/220V, is carried out. The system's parameters are given in Appendix A (Table A.1). The system is run under variable wind speed and rated load. The buck converter is controlled by a PI regulator in order to adjust the rotor speed to the reference speed $\left(\omega_{g, o p t}\right)$ where maximum power, available at each wind speed, is captured. Simulation results are shown in Fig. 3.5. Except for responses after $t=1 \mathrm{~s}$, MPPT controller works successfully by tracking the reference speed. At rated wind speed (12m/s) and with balanced excitation, the SCIG produces the rated power $(30 \mathrm{~kW})$ at rated line voltage $(320 \mathrm{~V})$ and frequency $(60 \mathrm{~Hz})$. Because no effort is made to control the inverter input dc current 
$\left(i_{d c}\right)$, it varies as the generator output does. Until $\mathrm{t}=0.3 \mathrm{~s}$, the minimum $i_{d c}$ required for the rated active load of $30 \mathrm{~kW}$, is guaranteed at the rated wind speed $(12 \mathrm{~m} / \mathrm{s})$. A unity modulation index produces the rated voltage $(380 \mathrm{~V} / 220 \mathrm{Vrms})$ at the load bus. At $\mathrm{t}=0.3 \mathrm{~s}$, the wind speed goes below the rated value; hence, $i_{d c}$ falls below its minimum value and $m_{i}$ saturates at its upper limit of 1 . As a result, the output voltage cannot be maintained at the desired level. At $t$ $=1 \mathrm{~s}$, the wind speed goes above the rated value. The MPPT controller is trying to extract the optimum wind power which exceeds the demand of the system. Because this topology has no mechanism to store or dump the excess power, the entire generated power will be transferred to the load irrespective of the demand. However, the CSI is trying to keep rated voltage across the constant-impendence load, which requires only rated current to flow in the load. In other words, the CSI works against the MPPT controller. As a result of this contradiction, the excess power is temporarily stored in the dc link reactor causing the dc-link current to rise uncontrollably, resulting in generator loss of excitation, as well as overvoltage and overcurrent at the load. In fact, with no mechanism of energy storage and/or dc-link current control, the system's behaviour under high wind speeds and/or light load is highly unpredictable and unsafe. On the contrary, if generated power is less than the demand, the load voltage will be lower than the desired value, which can also be harmful to the load.

One problem with using a diode rectifier as the generator-side converter is the resulting distortion in the stator current waveforms, leading to higher harmonic losses and torque ripples in the generator. Fig. 3.6 shows the generator stator current and electromagnetic torque at rated operating speed. It is noteworthy that harmonic distortion varies with generated frequency. The THD of the generator current at different wind speed is given in Table 3.1. The table shows high THDs in the generator current, especially at low wind speed corresponding to lowfrequency operation. In order to reduce the harmonic distortion, an L filter is typically installed on the generator side. 

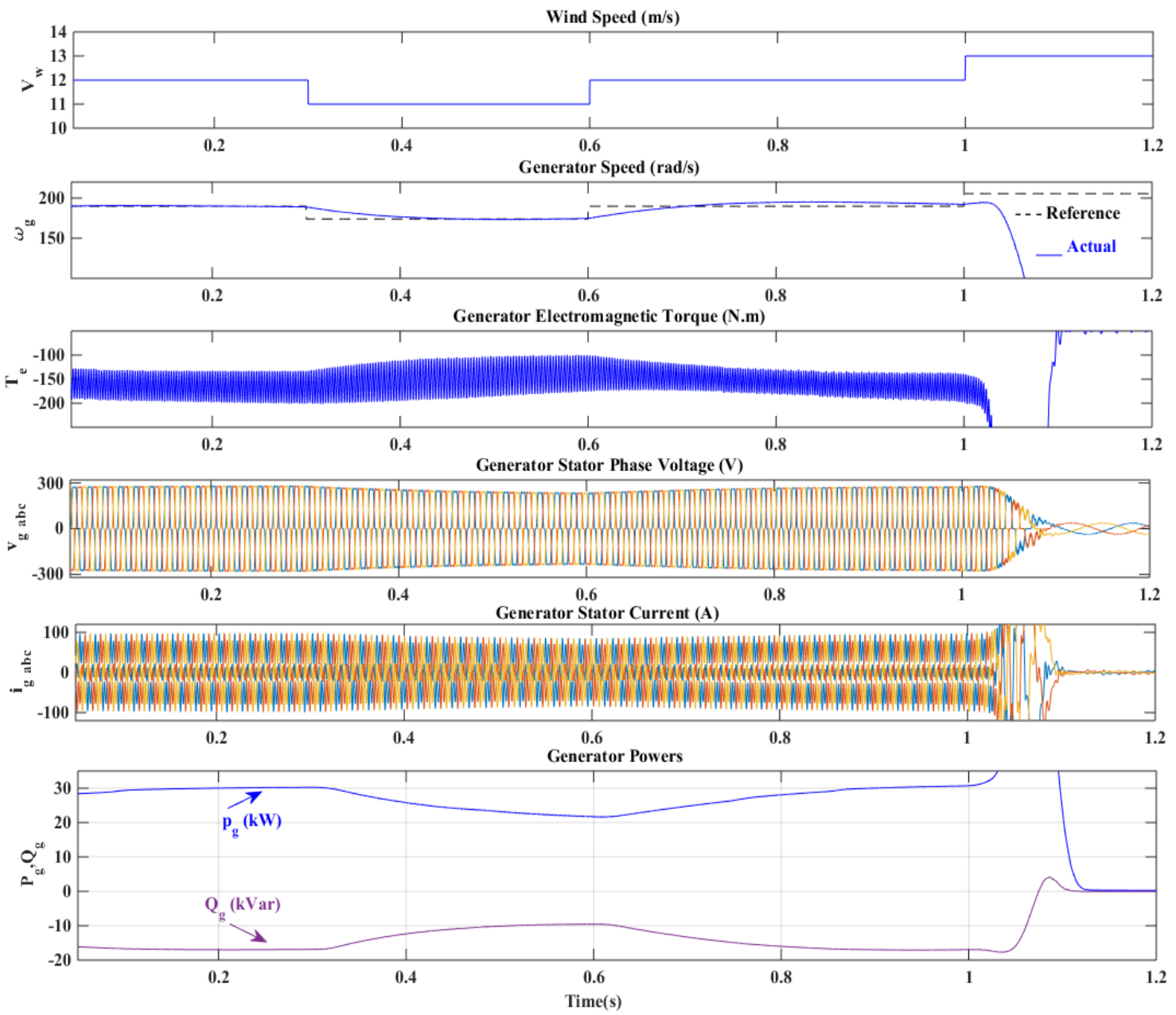

(a) Generator-Side Characteristics
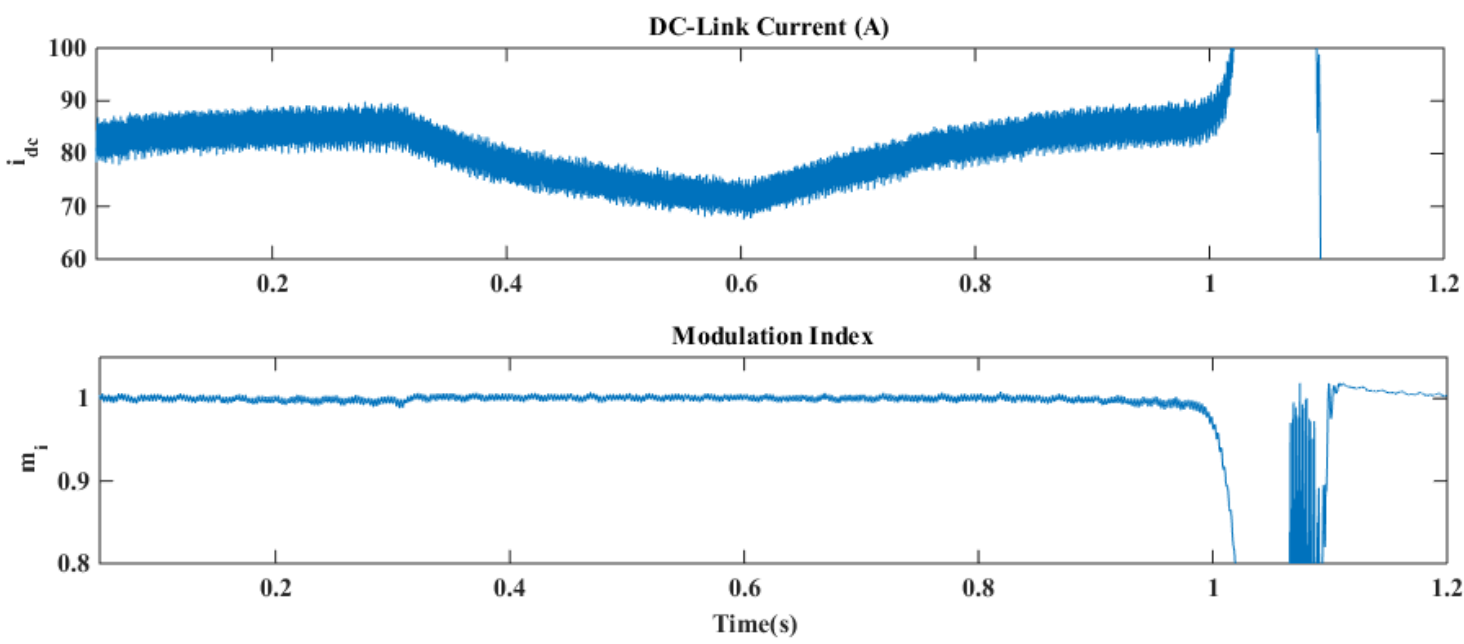

(b) DC-Link-Side Characteristics 

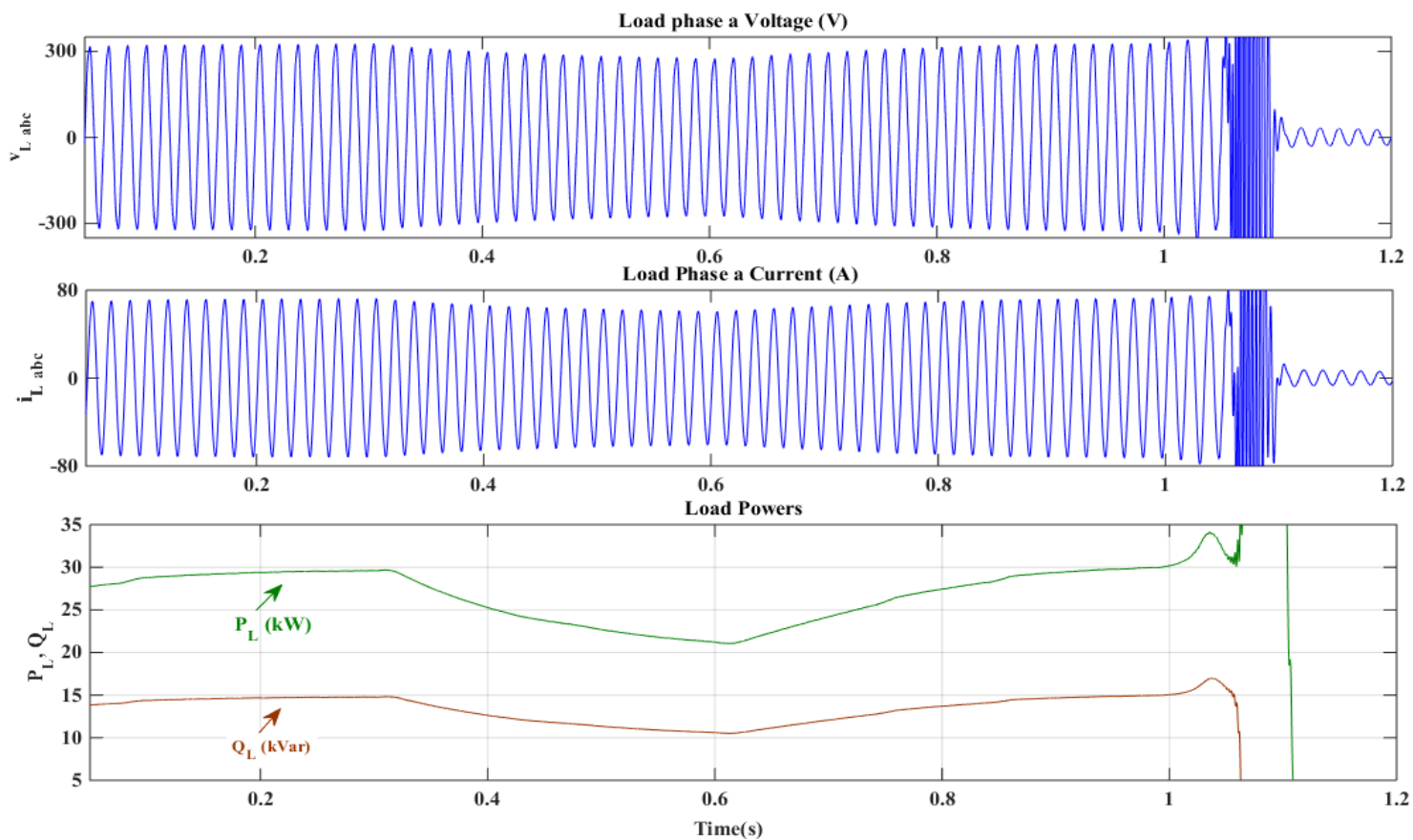

(c) Load-Side Characteristics

Fig. 3.5: Simulation results for topology 2.

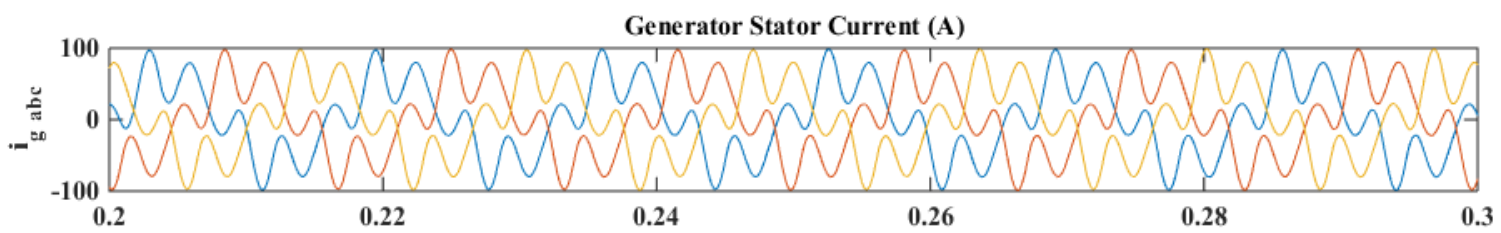

Generator Electromagnetic Torque (N.m)

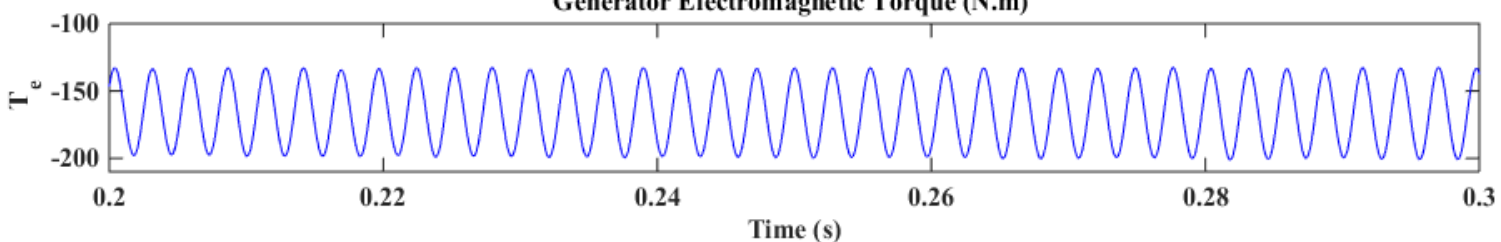

Fig. 3.6: Effect of diode bridge rectifier on generator characteristics at rated speed.

Table 3.1: THD of SCIG stator current in Topology 2.

\begin{tabular}{cc}
\hline Wind Speed $(\mathbf{m} / \mathbf{s})$ & \% \\
\hline 11 & 56.3 \\
12 & 45.2 \\
13 & 32.7 \\
\hline
\end{tabular}




\subsubsection{Topology 3: Back-to-Back CSC}

The drawbacks of using a diode bridge rectifier has been significantly reduced in the system illustrated in Fig. 3.7, where rectification is performed by a PWM-Current-Source Rectifier (PWM-CSR). Such a topology eliminates the need for the excitation capacitor bank because the reactive power, required by the SCIG, can be supplied from the PWM-CSR. However, capacitor bank $\left(C_{r}\right)$ is still required at the ac side of the PWM-CSR in order to filter out the switching harmonics in the line current and assist in the commutation of the rectifier switching devices. Nevertheless, size of $C_{r}$ is much smaller than that required for generator excitation in diode bridge rectifier configuration. $C_{r}$, combined with cable/generator inductance, forms a second-order LC filter, reducing the harmonic injected to the generator. $C_{r}$ 's design depends on PWM-CSR switching frequency, LC resonance, permitted line current THD, and generator type [15]. Similar to $C_{i}, C_{r}$ is designed under the assumption that the frequency of the lowest harmonic injected by the PWM-CSR is higher than the resonant frequency of the generatorside LC filter. The PWM-CSR is used to harvest the maximum power available from the wind, through regulating the generator torque or speed. The higher the CSR switching frequency is, the smaller the dc choke that is required. Compared with the case where a diode bridge rectifier is used, the dynamic performance of the generator is greatly improved by employing a PWM rectifier. The induction generator can be controlled by direct field oriented, indirect field oriented or direct torque control schemes. Direct rotor flux oriented control (DRFOC), implemented in Fig. 3.7, is one of the most common schemes used in WECS. The idea is to control the rotor flux $\left(\varphi_{r}\right)$ and electromagnetic torque $\left(T_{e}\right)$ independently. $\varphi_{r}$ is regulated to align with the $d$-axis rotor flux (i.e., $\varphi_{r}=\varphi_{d r}, \varphi_{q r}=0$ ), while $T_{e}$ is regulated to trace the generator's optimum torque $\left(T_{e, \text { opt }}\right)$ at each wind speed, leading to active power flow control. The reference $q$-axis stator current $\left(i_{q s, r e f}\right)$, required to produce optimum torque, is obtained based on equations (4.7) and (4.29 (c)) presented in Chapter 4. Rotor flux estimator block uses stator voltage $\left(v_{g_{a b c}}\right)$ and current $\left(i_{g_{a b c}}\right)$ to solve for stator flux, which can then be used along with stator current to calculate the rotor flux magnitude and angle. Further details on DRFOC can be found in [117]. 


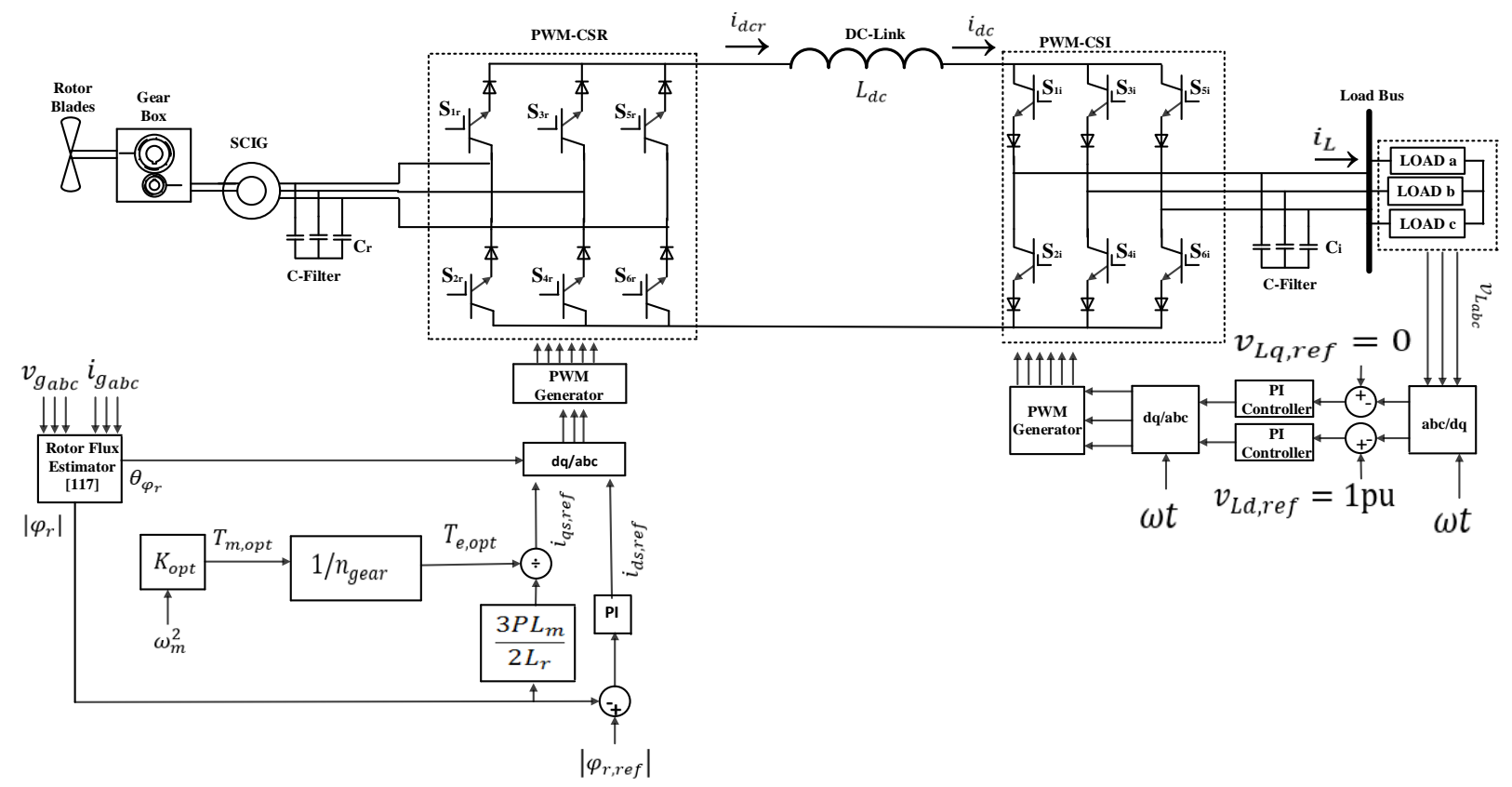

Fig. 3.7: A SCIG-WECS using back-to-back CSC.

To demonstrate the high performance of this topology, simulation of a $30 \mathrm{~kW} / 320 \mathrm{~V}$ standalone WECS, feeding a three-phase RL balanced load at 380V/220V, is carried out. The system's parameters are given in Appendix A (Table A.1). Fig. 3.8 shows that MPPT is achieved successfully by tracking the optimum electromagnetic torque at different wind speeds. As noticed in Fig. 3.9, the quality of generator's stator current is significantly improved (i.e., the current is nearly sinusoidal) when compared to topologies using a diode bridge rectifier as the generator-side converter. This will considerably reduce the generator harmonic losses and improve quality of generator torque. However, the advantage of lower generator harmonic contents is diminished by the losses of CSR switches. Since the DC-link provides an energy buffer between the generator-side and load- side converters, allowing for separate control of the converters on the two sides, the load-side characteristics, for this topology, are similar to those of topology 2 . The $i_{d c r}$ is regulated by the PWM-CSR; however, the $i_{d c}$ is still uncontrolled and hence it varies as the generator output does. As a result, minimum $i_{d c}$ is not guaranteed for the rated load at wind speeds below the rated value, leading to possibility of dclink current collapse and failure to supply the load. 

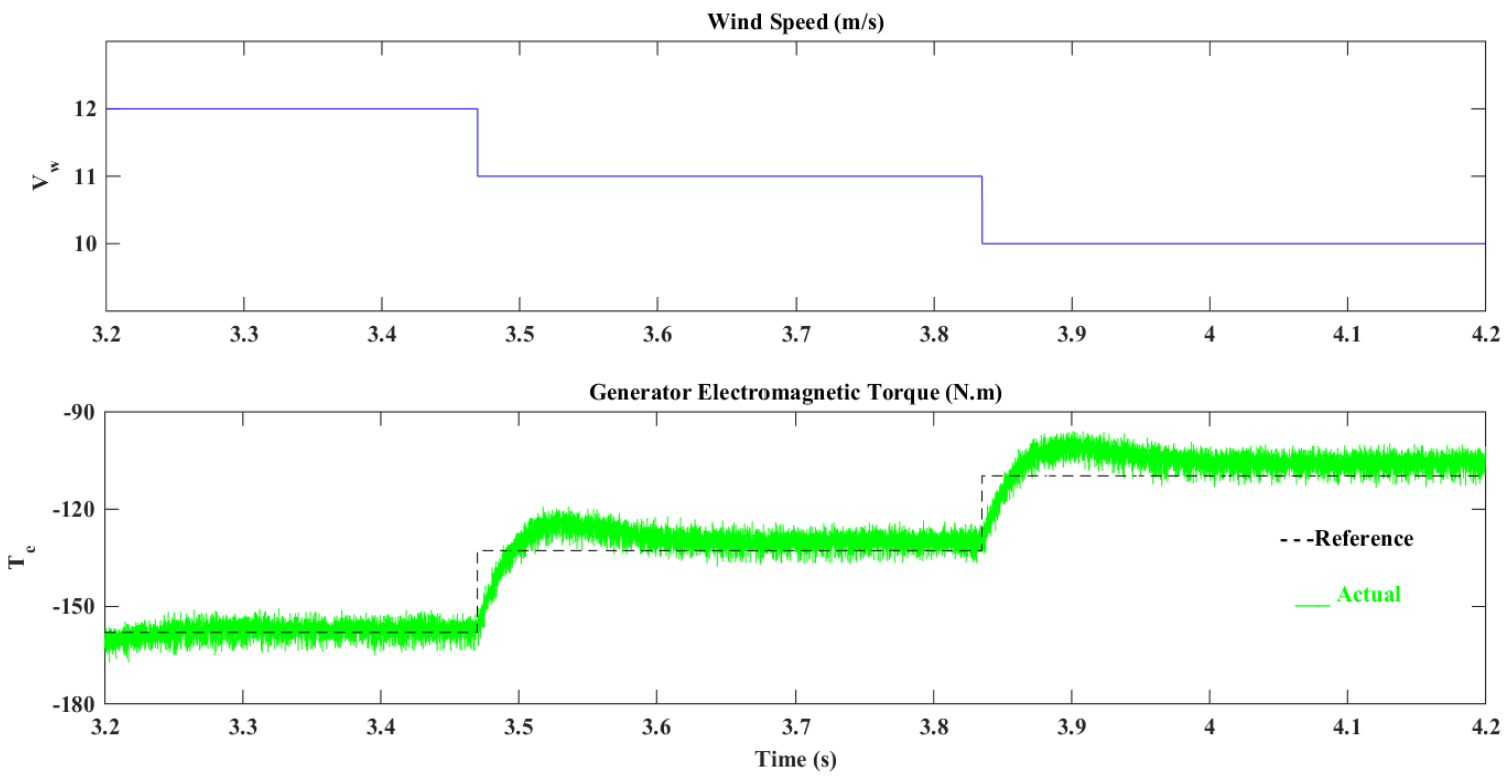

Fig. 3.8: MPPT for back-to-back CSC-based WECS.
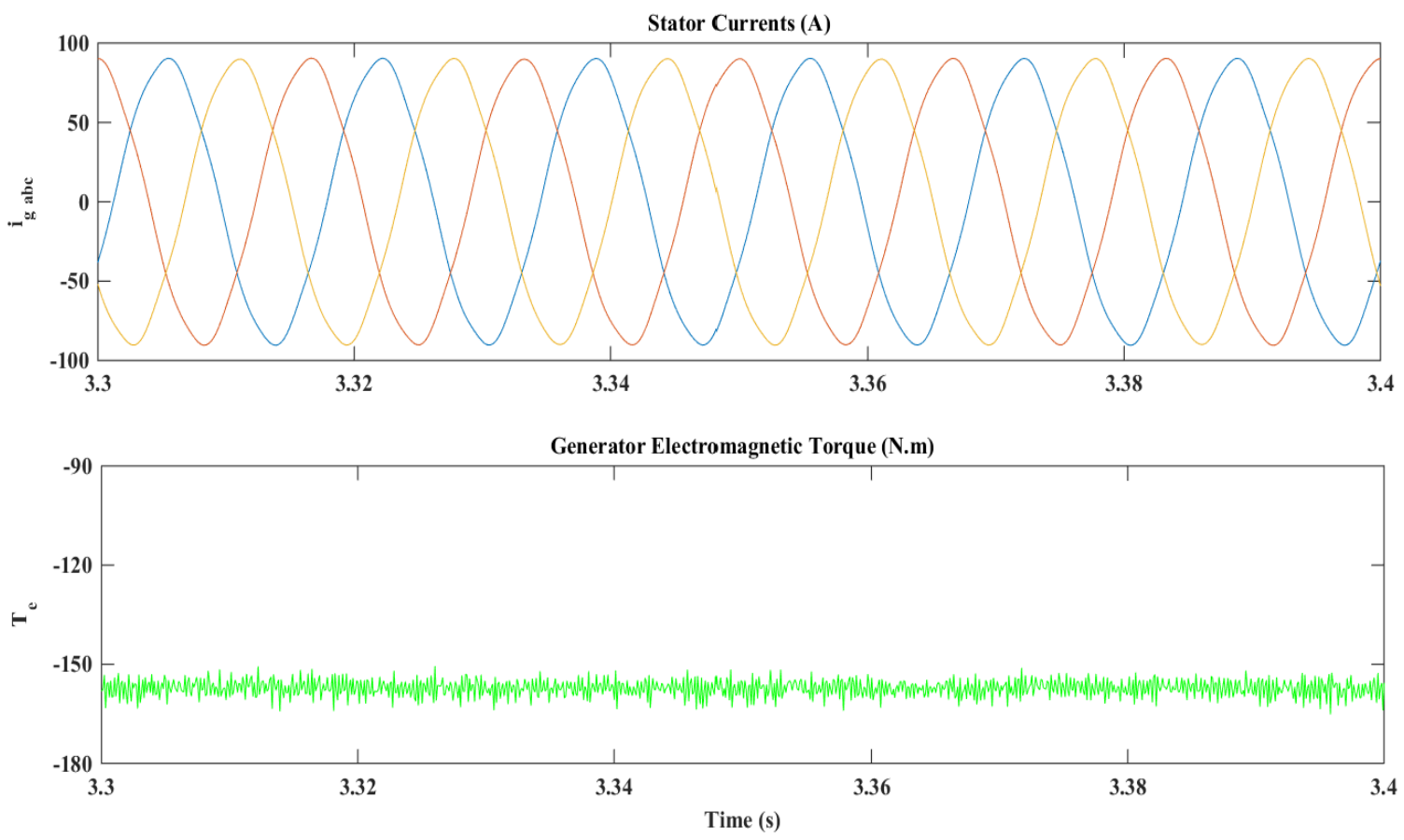

Fig. 3.9: Stator current and generator electromagnetic torque at rated speed. 


\subsection{Comparison of Different CSC-WECS Topologies}

Since it implements a diode bridge rectifier as the generator-side converter, the first topology (Fig. 3.2) provides no control over the generator, making MPPT impossible. Moreover, the two control degrees of freedom offered by CSI are used to control the voltage magnitude and frequency at the load bus. Thus, the dc-link current is left without control and hence the system behaviour is not predictable. This makes the first topology not applicable for standalone WECS. However, this configuration can be used for grid-connected WECS because the frequency is set by the grid and hence CSI can be used to control power factor at grid interface (through reactive power compensation, providing voltage support to the grid that might be weak at the point of connection) and dc-link current, according to a reference dictated by MPPT controller [74].

Adding a dc/dc buck converter to the output of generator-side diode bridge rectifier in the second topology (Fig. 3.4), introduces an additional control degree of freedom. It makes generator-side dc current controllable and MPPT achievable as long as the generated power doesn't exceed the demand of the system. However, the minimum load-side dc current is still not guaranteed at all wind speeds. Another problem associated with this topology is the nonlinear characteristics of the diode bridge rectifier, introducing high harmonic distortion to the generator winding currents, leading to high harmonic losses and torque ripples in the generator. Nevertheless, this harmonic distortion can be attenuated by installing an L filter in series with generator.

The back-to-back CSC configuration (Fig. 3.7) improves the generator performance significantly, but with a complex control, as well as higher cost and converter losses due to higher number of switching devices. Moreover, although the MPPT is achievable, the minimum value of the inverter input dc current, required to maintain the output voltage, is not guaranteed.

A brief comparison of the three topologies described above, is given in Table 3.2. As a simple and low-cost configuration, offering satisfactory performance, topology 2 (i.e., diode bridge rectifier - buck converter - PWM CSI) is selected as the base for detailed studies in this thesis. 
Table 3.2: Comparison of the three standalone CSC-based WECS topologies.

\begin{tabular}{llll}
\hline Comparison item & $\begin{array}{l}\text { Diode Rectifier + } \\
\text { PWM CSI }\end{array}$ & $\begin{array}{l}\text { Diode Rectifier + } \\
\text { Buck Converter } \\
\text { + PWM CSI }\end{array}$ & $\begin{array}{l}\text { Back-to-back } \\
\text { CSC }\end{array}$ \\
\hline Converter cost & Lowest & Low & High \\
Control Degrees of freedom & 2 & 3 & 4 \\
Generator-side harmonics & High & High & Low \\
Excitation Capacitor & Required & Required & Not required \\
MPPT & Not applicable & Achievable & Achievable \\
Minimum $i_{d c}$ requirement & Not applicable & Not guaranteed & Not guaranteed \\
Dynamic performance & Not applicable & Medium & High \\
\hline
\end{tabular}

\subsection{Integration of Energy Storage with the CSI}

Storage integration within VSI-based WECS has received a great deal of attention from both researchers [32],[47],[65] and manufacturers [113]. However, since the CSI-based WECSs are usually employed for on-grid applications, storage integration has not been a real concern, neither in the literature nor in the wind market. Reference [70] designed a simple energystorage circuit to be added to a standalone small-scale generation system. The purpose of the circuit was to handle the turn-on transient events in the load fed through a CSI. This is done by temporarily increasing the system input power as loading increases. A capacitor was used as the temporary storage device. To evaluate the feasibility of such a circuit for a permanent storage purposes, it was implemented in topology 2, as shown in Fig. 3.10, but the capacitor bank was replaced by a storage battery. The idea is to charge the battery during zero states of the CSI. This is done by modifying the gating pattern of PWM-CSI in order to open all the switches during the zero states only, such that the dc-link current is forced to flow through the diode $D_{c}$ and charge the battery. To ensure that $D_{c}$ will not conduct during CSI's active states, the battery nominal voltage $\left(v_{\text {bat }}\right)$ should be higher than the peak inverter input voltage $\left(v_{\text {inv }}\right)$, 
which is equal to the peak line voltage at unity modulation index. This condition can be stated as $v_{\text {bat }}>\widehat{V}_{L L}$.

A Lead Acid Battery (LAB) is used in this simulation. Considering a daily demand of $D_{L}$ $\mathrm{kWh}$ for $u$ back-up days with no wind power (determined based on the wind profile at the installation site and the load profile), battery rated voltage of $V_{\text {bat,rated }}$, depth of discharge of $D O D$ and temperature factor of $k_{T}$, the required Ampere-hour (Ah) capacity of battery bank can be found as

$$
A h=\frac{\left(D_{L}\right)(u)\left(k_{T}\right)}{(D O D)\left(V_{\text {bat }, \text { rated }}\right)}
$$

For $D_{L}=30 \mathrm{kWh}, u=1, V_{\text {bat,rated }}=650 \mathrm{~V}, \mathrm{DOD}=50 \%$, and $k_{T}=1\left(\right.$ at $25^{\circ} \mathrm{C}$ ), the Ah capacity of the battery bank is around $92 \mathrm{Ah}$. Thus, 54 standard commercial 12V, 92Ah batteries are connected in series to produce a total storage capacity of $59.6 \mathrm{kWh}$.

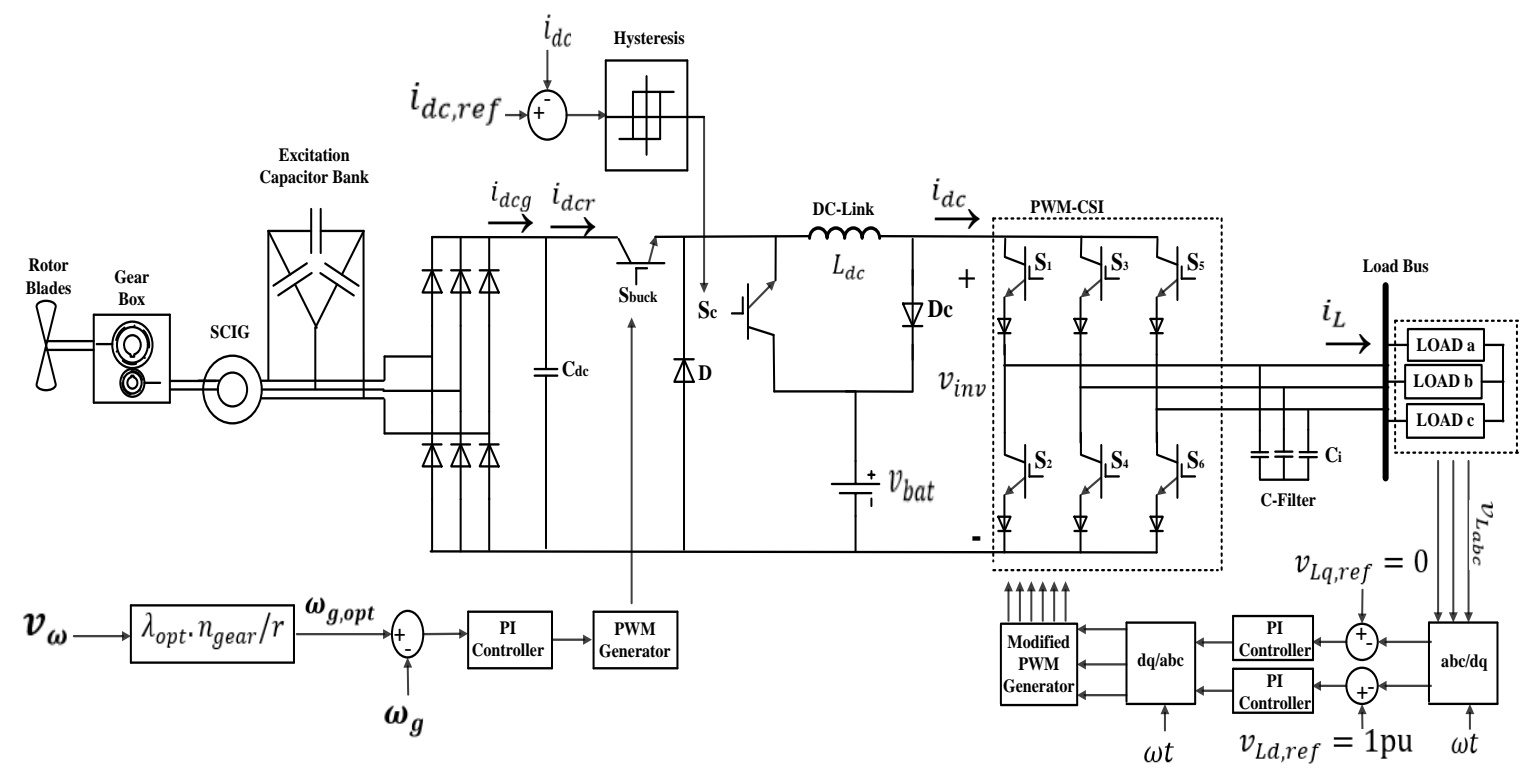

Fig. 3.10: Integration of the energy storage system proposed in [70] with the CSI- based WECS of topology 2.

Simulation responses are shown in Fig. 3.11. The system starts running at rated wind speed $(12 \mathrm{~m} / \mathrm{s})$, feeding rated RL load. The demand is reduced to $70 \%, 50 \%$ and $20 \%$ of the rated load 
at $\mathrm{t}=1 \mathrm{~s}, 1.5 \mathrm{~s}$, and $3 \mathrm{~s}$, respectively. At $\mathrm{t}=3.3 \mathrm{~s}$, the rated load is applied again. The wind speed is changed to 13 and 14 at $\mathrm{t}=2 \mathrm{~s}$ and $2.5 \mathrm{~s}$, respectively. MPPT control is achieved successfully, as illustrated in Fig. 3.11. The figure also demonstrates a good power distribution among wind generator, battery and load during transients and steady-state conditions. If wind power is lower than the power demand, as in the start-up period ( $\mathrm{t}=0 \mathrm{~s}$ to $\mathrm{t}=0.16 \mathrm{~s})$, the battery compensates the shortage in power through switch $S_{c}$ and hence the dc-link current $\left(i_{d c}\right)$ is guaranteed not to fall below its reference. Although the diode $D_{c}$ offers no control over the dclink current, the fact that the battery is periodically charged during zero states of the CSI can help in reducing dc-link current if it exceeds its reference, when the available wind power is higher than the load demand. This can be seen in the period from $t=1 \mathrm{~s}$ to $2 \mathrm{~s}$. Since wind power is higher than demand during most of the simulation time, the battery's SoC is set to low value (i.e., 25\%) initially so that the battery has enough room for charging. As the excess of wind power increases during the period $\mathrm{t}=2 \mathrm{~s}$ to $3.5 \mathrm{~s}$, the $i_{d c}$ can get much higher than the reference and the storage circuit will not be able to maintain it. Even if a controlled switch is used instead of the diode $D_{c}$, the maximum value of $i_{d c}$ is still unregulated because the charging process can only take place during the zero states of the inverter. Even though $i_{d c}$ exceeds its reference, this case is still accepted for the load-side because the modulation index will adjust the CSI output current. However, as $i_{d c}$ increases further, lower values of $m_{i}(<0.5)$ results in distortion of the output voltage waveform, as can be noticed from $\mathrm{t}=3 \mathrm{~s}$ to $\mathrm{t}=3.3 \mathrm{~s}$. Moreover, high $i_{d c}$ produces higher $i_{d c}^{2} R_{d c}$ and inverter losses. Therefore, this storage circuit may be used in a standalone WECS only if the load demand and wind speed ranges are restricted to produce small value of extra power that can be handled by the frequent battery charging, taking place during zero states of the inverter. However, restricting wind power conflicts with the MPPT objective. To overcome the shortcomings of the energy storage system proposed in [70], a novel scheme for integration of a battery energy storage system with the CSI-based WECS of topology 2 is proposed in the following section. 

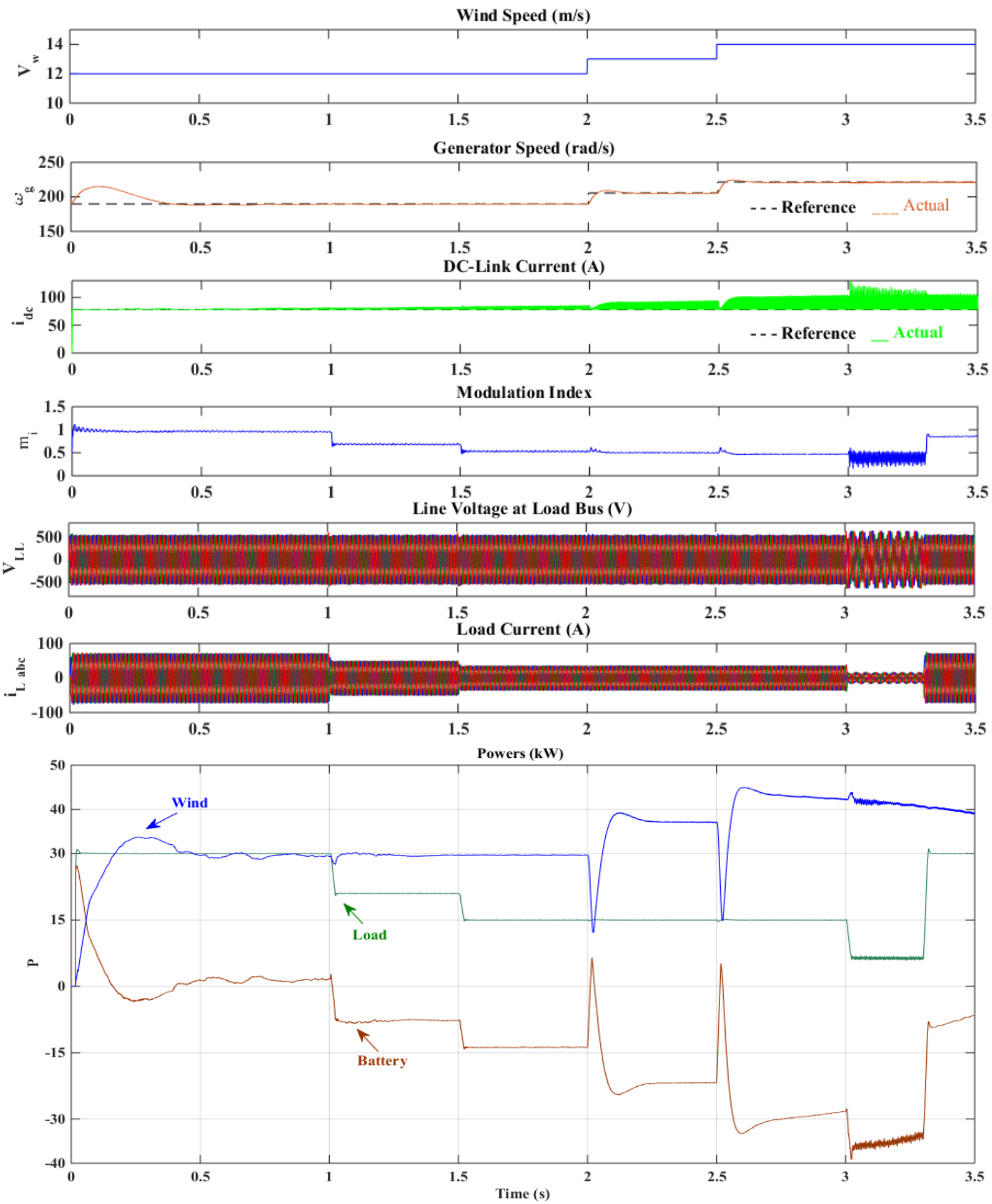

Fig. 3.11: Simulation results for the CSI-based WECS with energy storage system proposed in [70]. 


\subsection{The Structure of the Proposed WECS}

In this work, the duality of CSI and VSI topologies has been taken advantage of to come up with a novel scheme for the integration of a battery-based energy storage system with the proposed CSI-based WECS. In standalone VSI-based WECS, the voltage across the dc-link capacitor is regulated by connecting a battery bank across the dc-link filter capacitor via a bidirectional buck-boost dc/dc converter [49], with bidirectional-current and unipolar-voltage capabilities, and controlling the converter to manage the power exchange between the battery and the dc-bus. In CSI-based WECS to exchange power between the battery and the dc bus, an interfacing converter with bipolar-voltage and unidirectional-current capabilities is required. As shown in Fig. 3.12, this requirement has been fulfilled by employing a full-bridge $\mathrm{dc} / \mathrm{dc}$ converter (H-bridge) with reduced number of switches, which is simply referred to as reduced $\mathrm{H}$-bridge.

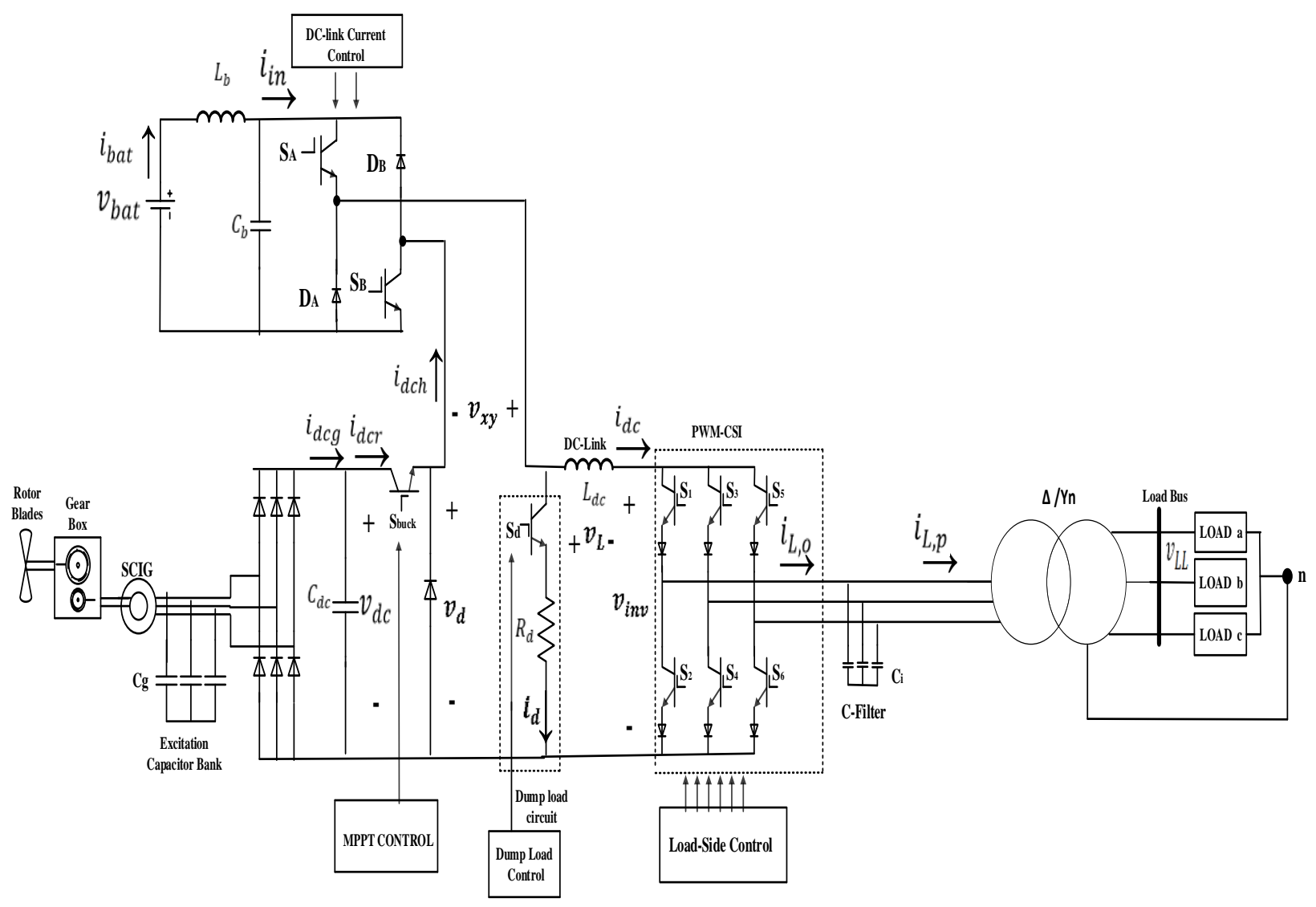

Fig. 3.12: Structure of the proposed WECS. 
As shown in Fig. 3.12, the proposed SCIG-based WECS employing a PWM CSI consists of the following components:

- Rotor blades,

- Geared-drive, self-excited squirrel-cage induction generator,

- Three-phase diode bridge rectifier,

- DC/DC buck converter,

- DC-Link inductor,

- IGBT PWM three-phase current-source inverter,

- C-Filter $\left(C_{i}\right)$,

- Delta/star $(\Delta / Y n)$ transformer, providing a path for zero sequence current, isolating the load from the system, and protecting the motoring load from common mode voltage,

- Y-connected three-phase load,

- Storage battery integrated with the dc-link via a reduced H-bridge dc/dc converter, and

- Dump load.

The main objectives of the controller design are:

1. To achieve maximum power point tracking (MPPT),

2. To achieve effective control coordination among the wind generator, battery, and dump load to maintain the dc-link current at the desired value, and

3. To maintain balanced voltages, with constant magnitude and frequency, at the load bus.

A standalone WECS, employing a diode rectifier, a dc/dc boost converter, a VSI and a bidirectional buck-boost dc/dc converter as the interface of the storage battery with the system, requires two inductors (one for the boost converter and one for the buck-boost converter), and a dc-link capacitor. In the proposed CSI-WECS, in contrast, the dc-link inductor $\left(L_{d c}\right)$ is shared by the buck converter, reduced H-bridge and CSI, resulting in reduction of the system size, weight and cost. A systemic procedure for design of $L_{d c}$ is introduced in the following subsection. 


\subsubsection{Systematic Design of the DC-Link Inductor}

DC-link inductor $\left(L_{d c}\right)$ is sized according to the specified upper limit for the ripple in the dclink current at steady state. The study of the voltage that is placed across the dc-link inductor $\left(v_{L}\right)$, under different operating conditions, is the key to the design of $L_{d c}$. Applying KVL to dc-link loop in Fig. 3.12, $v_{L}$ can be expressed as

$$
v_{L}=v_{d}+v_{x y}-v_{i n v}
$$

where $v_{d}$ is the buck converter unfiltered output voltage, $v_{x y}$ the voltage inserted by the reduced H-bridge in the dc-link and $v_{i n v}$ the CSI's dc-side voltage. The instantaneous values assumed by $v_{d}, v_{x y}$ and $v_{i n v}$ depend on the operating conditions of buck converter, reduced $\mathrm{H}$ bridge and CSI, respectively, and are given in Table 3.3, where $v_{d c}, v_{\text {bat }}$ and $v_{L L}$ represent the rectified dc voltage, the battery voltage, and the load-side line voltage, respectively. For simplicity, the instantaneous value of the rectified voltage has been approximated by its average value.

Table 3.3: Possible values of $v_{d}, v_{x y}$, and $v_{i n v}$.

\begin{tabular}{|c|c|c|}
\hline Voltage & Value & Operation condition \\
\hline$v_{d}$ & $\begin{array}{l}v_{d c} \\
0\end{array}$ & $\begin{array}{l}S_{\text {buck }} \text { is ON } \\
S_{\text {buck }} \text { is OFF }\end{array}$ \\
\hline$v_{x y}$ & $\begin{array}{c}v_{\text {bat }} \\
-v_{\text {bat }} \\
0\end{array}$ & $\begin{array}{l}\text { Battery is discharging } \\
\text { Battery is charging } \\
\text { Battery is neither charging nor } \\
\text { discharging (freewheeling state) }\end{array}$ \\
\hline$v_{i n v}$ & $\begin{array}{c}v_{L L} \\
0\end{array}$ & $\begin{array}{l}\text { CSI is in one of the active states } \\
\text { CSI is in one of zero (shoot-through) } \\
\text { states }\end{array}$ \\
\hline
\end{tabular}

The maximum instantaneous voltage that is applied across $L_{d c}\left(V_{L, \max }\right)$ is

where

$$
V_{L, \text { max }}=v_{d c, \text { max }}+v_{b a t}+\widehat{V}_{L L}
$$




$$
v_{d c, \max }=\frac{3 \sqrt{2}}{\pi} V_{g, L L, \max }
$$

and $V_{g, L L, \max }$ is the maximum rms value of SCIG line voltage.

On the other hand, the minimum instantaneous voltage that is applied across the dc-link inductor $\left(V_{L, \min }\right)$ is

$$
V_{L_{\min }}=0-v_{b a t}-\widehat{V}_{L L}
$$

As shown in Fig. 3.13, the dc-link inductor current rises and falls in response to application of positive and negative voltages across the dc-link inductor, respectively. The extreme case for the range of variation of $v_{L}$ corresponds to the rare condition where $V_{L, \max }$ and $V_{L, \min }$ are applied across dc-link inductor successively. Under this condition, the maximum rise, during $\Delta t_{1}$, and maximum fall, during $\Delta t_{2}$, of the dc-link current can be obtained from (3.6) and (3.7), respectively, as

$$
\begin{gathered}
\Delta i_{d c, \text { rise,max }}=\frac{V_{L, \text { max }}}{L_{d c}} \Delta t_{1} \\
\Delta i_{d c, \text { fall,max }}=\frac{\left|V_{L, \text { min }}\right|}{L_{d c}} \Delta t_{2}
\end{gathered}
$$

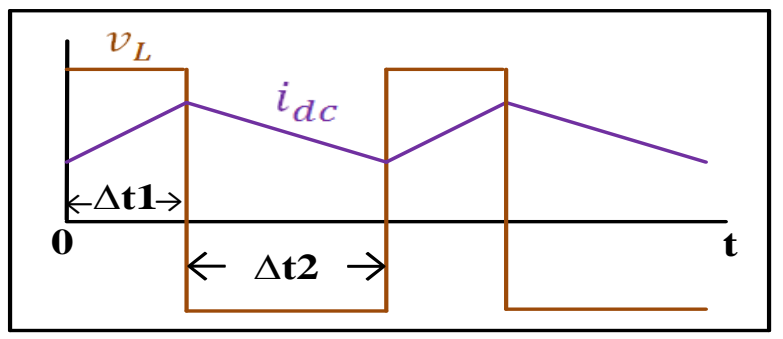

Fig. 3.13: DC-link inductor voltage and current.

At steady state, the inductor current is repetitive and the values of inductor current rise and fall are equal. Therefore,

$$
\Delta i_{d c, \text { rise } \max }=\Delta i_{d c, \text { fall }, \max }=\Delta i_{d c}
$$

Assuming the same switching frequency $\left(f_{S}\right)$ for the buck converter, reduced H-bridge and CSI, one can write 


$$
\Delta t_{1}+\Delta t_{2}=\frac{1}{f_{s}}
$$

Based on (3.6) - (3.9), the worst-case steady-state ripple in dc-link inductor current can be expressed as

$$
\Delta i_{d c}=\frac{\left|V_{L, \min }\right|}{L_{d c} f_{s}\left(1+\frac{\left|V_{L, \min }\right|}{V_{L, \max }}\right)}
$$

The inductor current ripple is normally expressed relative to the average value of the inductor current. Thus,

$$
\frac{\Delta i_{d c}}{i_{d c}}=\frac{\left|V_{L, \min }\right|}{L_{d c} f_{S}\left(1+\frac{\left|V_{L, \min }\right|}{V_{L, \max }}\right) i_{d c}}
$$

Typically, dc-link current ripple in CSI is limited to 15\% [15]. From (3.11), the minimum inductance required to satisfy this limit is

$$
L_{d c_{\text {min }}}=\frac{\left|V_{L, \min }\right|}{f_{s}\left(1+\frac{V_{L, \text { min }} \mid}{V_{L, \text { max }}}\right)\left(0.15 i_{d c}\right)}
$$

It can be noticed from (3.12) that the size of dc-link inductor can be reduced by increasing the switching frequency. Moreover, the higher the dc-link current is, the smaller the inductance required to guarantee not exceeding the specified current ripple limit will be. However, it is useful to minimize the dc-link current so as to reduce the ohmic losses of the dc-link inductor as well as the losses of the current source inverter. The minimum dc-link current based on which the dc-link inductor is designed will be specified in Chapter 5 when dc-link current control system is designed.

\subsection{Summary}

In this chapter, the feasibility of employing CSI in off-grid WECS was investigated. The three CSC topologies, validated in the literature for on-grid WECS, were evaluated for off-grid WECS application. Since the first topology (i.e., diode bridge rectifier - PWM CSI) provides no control over generator and dc-link current, it is not applicable for an off-grid WECS. Adding an intermediate dc/dc buck converter to the first topology, the second topology (i.e., diode bridge rectifier - buck converter - PWM CSI) makes MPPT achievable. One problem 
associated with diode bridge rectifier-based topologies is the high harmonic distortion in the generator winding current. This problem was overcome by employing PWM-CSR in the third topology (i.e., Back-to-back PWM CSC), leading to significant improvement in the generator performance, but with complex control and at higher cost. As a simple and low-cost configuration, offering satisfactory performance, the second topology was selected as the base for further study in this research.

Although the feasibility of the selected topology was demonstrated, the minimum value of the CSI input dc current, required to maintain desired output voltage, was not guaranteed at wind speeds below rated value. Moreover, a storage unit was required to be integrated within the structure of the system in order to achieve effective power balance. A simple storage circuit, proposed in the literature for temporary storage purposes, was implemented in the second topology, but the capacitor bank was replaced by a storage battery. The validity of such a simple storage circuit, for standalone turbine, was demonstrated only when the load and wind speed ranges are restricted to produce small value of extra power; this implies restricting wind power and conflicts with the MPPT objective. Therefore, a novel scheme for integration of a battery energy storage system with the CSI-based WECS of the second topology, was proposed. The dc-link inductor, shared by three converters, was systematically designed. 


\section{Chapter 4}

\section{Dynamic Modeling and Small Signal Analysis of the CSI-WECS}

Fig. 4.1 shows the block diagram of the proposed system. In this chapter, detailed models of mechanical and electrical components are developed and combined to form an overall model.

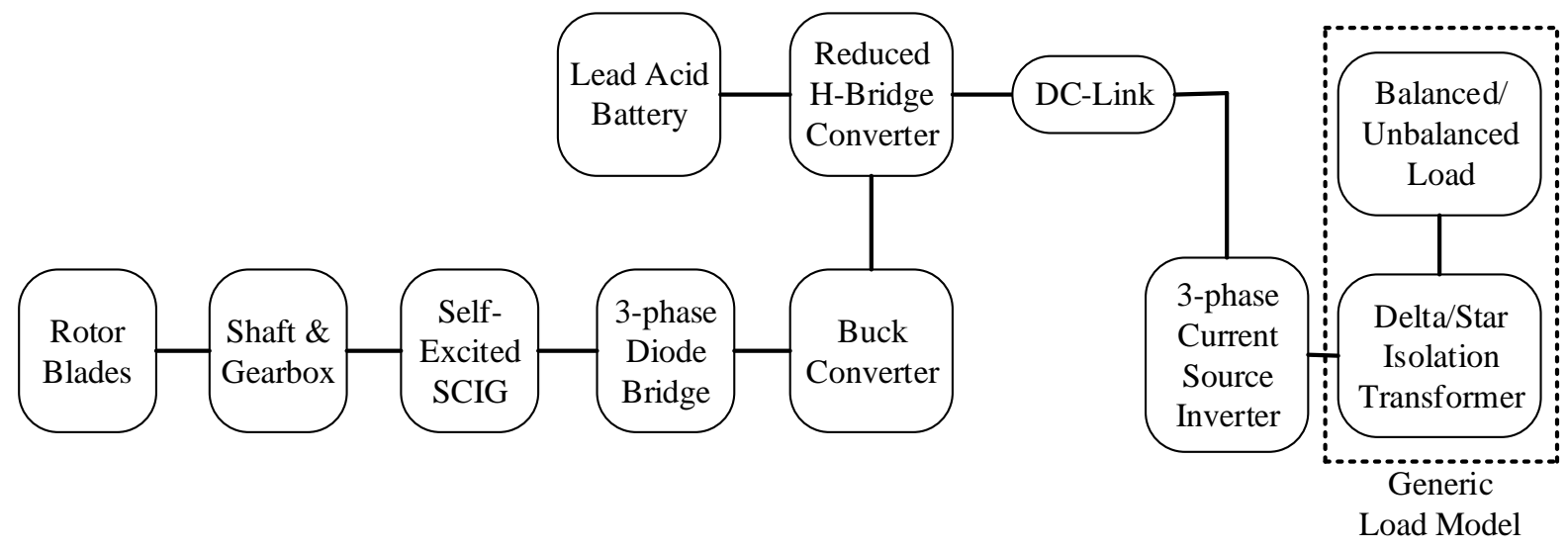

Fig. 4.1: Block diagram of the proposed CSI-based WECS.

Forced-commutated power converter devices operate at high frequencies and hence a very small simulation time step is required to produce a sufficiently accurate simulation of switching transients. Therefore, simulating the entire system using a real switching model will demand a long simulation time and a large memory size. Moreover, the discontinuities and nonlinearities associated with the switching action of the power converters make it complicated to apply classical control methods for system analysis.

When switching ripples are of interest or detailed transient information is needed, detailed switching models are unavoidable; otherwise, average models can provide adequate information in a low frequency range [118]. High-frequency switching harmonics are not presented in the average model, but the dynamics of the system is preserved. Average model, which predicts low-frequency behaviour of the actual switching model, can be simulated much faster than switching model. Assuming that the smallest time constant of a converter system is larger than the switching period $\left(T_{s}\right)$ by at least an order of magnitude, it is a very good approximation to average the converter variable quantities over $T_{s}$. As a result, the switching action is eliminated from the model and hence system's discontinuity is no longer a concern. 
In other words, the average model is continuous and hence can be linearized around an operating point. The linearized model is used to investigate local stability, observability and controllability of the system. The linearized model also provides the basis for closed-loop control system design in the following chapter.

Typically, the process of modeling starts with deriving three-phase equations for electrical circuits in abc frame. These relations are then transformed into their corresponding $d q$-frame equations. The transformation matrix is given in (4.1), where $x$ can be any system variable such as three-phase voltage, current, and flux linkage, and $\omega t$ is the angular position of the rotating $d q$ frame in radians.

$$
\left[\begin{array}{l}
x_{d} \\
x_{q}
\end{array}\right]=\frac{2}{3}\left[\begin{array}{ccc}
\cos (\omega t) & \cos \left(\omega t-\frac{2 \pi}{3}\right) & \cos \left(\omega t+\frac{2 \pi}{3}\right) \\
-\sin (\omega t) & -\sin \left(\omega t-\frac{2 \pi}{3}\right) & -\sin \left(\omega t+\frac{2 \pi}{3}\right)
\end{array}\right]\left[\begin{array}{l}
x_{a} \\
x_{b} \\
x_{c}
\end{array}\right]
$$

Since the proposed WECS has some passive components, which can be described using differential equations, an additional term is brought to the transformation, as seen in (4.2), where $\omega$ is the angular speed of the $d q$ frame.

$$
\frac{d}{d t}\left[\begin{array}{l}
x_{a} \\
x_{b} \\
x_{c}
\end{array}\right] \Rightarrow \frac{d}{d t}\left[\begin{array}{l}
x_{d} \\
x_{q}
\end{array}\right]+\left[\begin{array}{cc}
0 & -\omega \\
\omega & 0
\end{array}\right]\left[\begin{array}{l}
x_{d} \\
x_{q}
\end{array}\right]
$$

In this chapter:

1) The models of system components are derived and combined to develop a nonlinear dynamic model of the proposed standalone CSI-based WECS in $d q$ reference frame. Detailed models of the aerodynamic conversion, drive train, self-excited induction generator, Lead Acid battery, and power-electronic converters are presented and combined with a reduced-order generic load model to enable transient and steady-state analyses of the overall system;

2) The behavior of the system is investigated by simulating its operation at start-up and in response to a step change in the input;

3) A small-signal linear model is developed by linearizing the nonlinear dynamic equations around steady-state selected operating points; and 
4) The linearized model is employed to investigate the local stability of the system at the steady-state operating point. Also, some of the system properties, such as controllability and observability are investigated.

\subsection{Wind Turbine System}

The mechanical power captured by a wind turbine $\left(P_{m}\right)$ can be obtained from

$$
P_{m}=\frac{\rho A}{2} v_{w}^{3} C_{p}(\lambda, \beta)
$$

where $\rho$ is the air density $\left(1.225 \mathrm{~kg} / \mathrm{m}^{3}\right.$ at $15^{\circ} \mathrm{C}$ and at sea level), $A$ the turbine's swept area in $m^{2}\left(A=\pi r^{2}\right.$, with $r$ the radius of blades in $\left.m\right), v_{w}$ the wind speed in $m / s, C_{p}$ the wind turbine performance coefficient, $\lambda$ the tip speed ratio, and $\beta$ the blade pitch angle in degrees. $\lambda$, defined as the ratio of rotor blade tip speed to wind speed, is

$$
\lambda=\frac{\omega_{m} r}{v_{w}}
$$

where $\omega_{m}$ is the angular speed of the turbine in $\mathrm{rad} / \mathrm{s}$.

$C_{p}$ is a function of $\lambda$ and $\beta$, as given in (4.5) [119].

$$
C_{p}(\lambda, \beta)=0.5176\left(\frac{116}{\lambda_{i}}-0.4 \beta-5\right) e^{-\frac{21}{\lambda_{i}}}+0.0068 \lambda
$$

In (4.5),

$$
\frac{1}{\lambda_{i}}=\frac{1}{\lambda+0.08 \beta}-\frac{0.035}{\beta^{3}+1}
$$

According to Betz law, the theoretical limit of $C_{p}$ is 0.59 . Practically, $C_{p}$ varies between 0.2 and 0.5 in the modern turbines [15]. The $C_{p}-\lambda$ characteristics for different $\beta$ values are illustrated in Fig. 4.2 for a three-blade horizontal-axis WT. The maximum $C_{p}\left(C_{p_{\max }}\right)$, for $\beta=$ 0 , is obtained at $\lambda=8.1$. This particular $\lambda$ is known as optimal $\lambda\left(\lambda_{o p t}\right)$. For a variable-speed WT, with the pitch angle fixed at zero, the target is to maintain $\lambda$ at its optimal value corresponding to maximum power capture. 


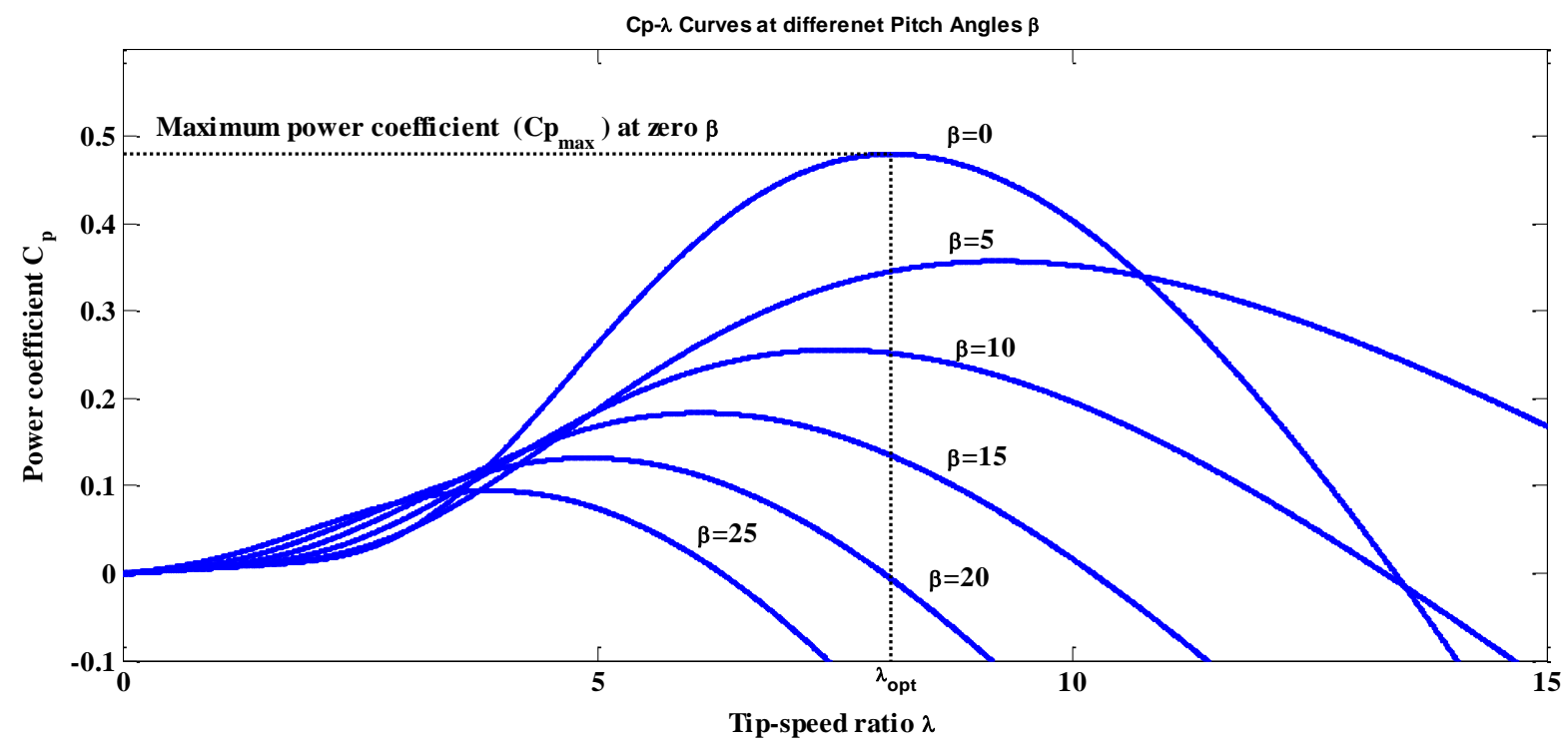

Fig. 4.2: Performance coefficient versus tip speed ratio for various blade pitch angles.

Fig. 4.3 shows the steady-state mechanical power-speed curves for a variable-speed, fixedpitch WT in per unit. The curves show the nonlinear relationship between the mechanical power and wind speed. The red dashed line links the optimum power points at different wind speeds. The rotor speed corresponding to the maximum power at each wind speed can be found based on the optimal tip speed ratio from (4.4). Fig. 4.3 is obtained for base wind speed of 12 $\mathrm{m} / \mathrm{s}$ and rated power of 1 pu at 1 pu rotational speed.

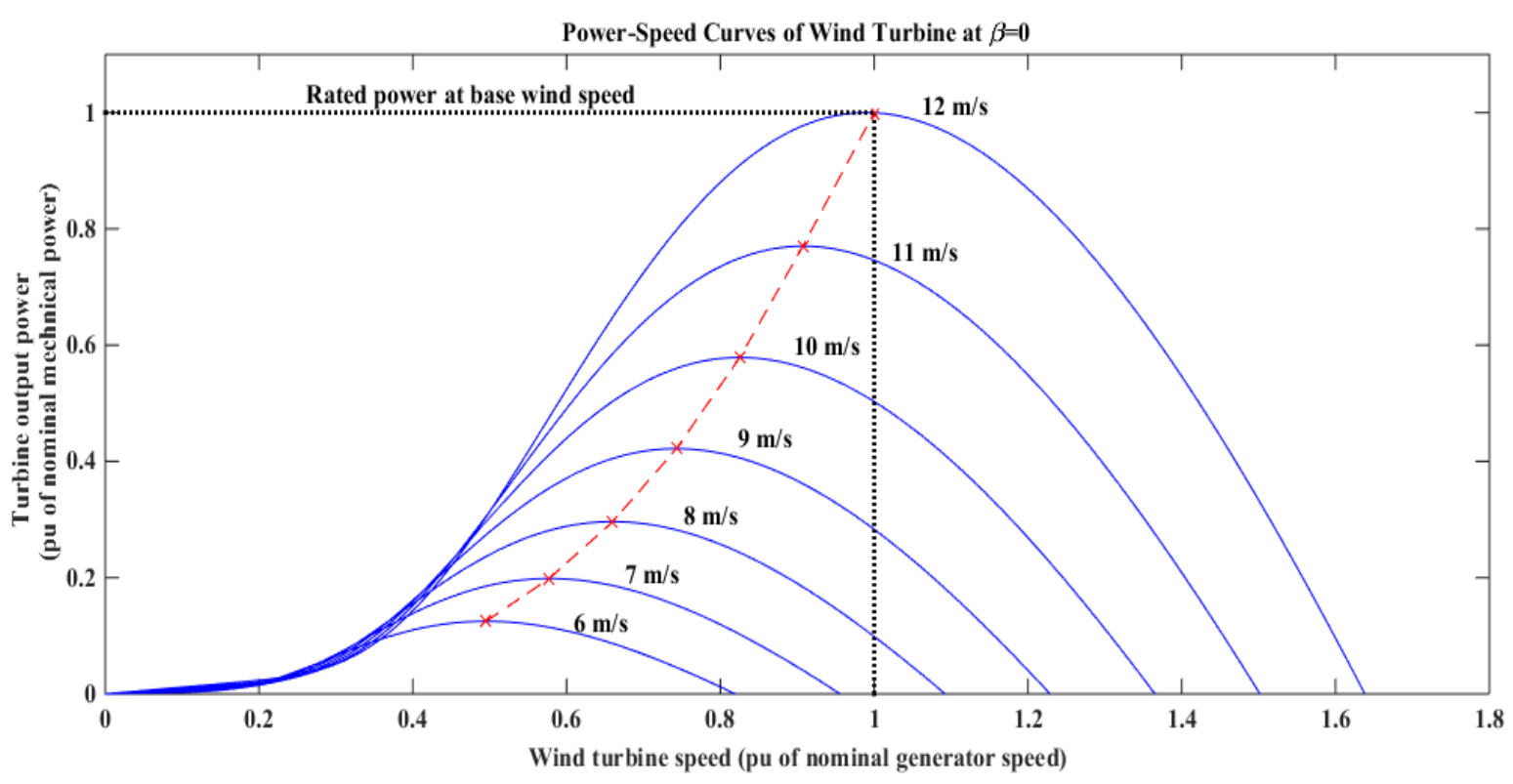

Fig. 4.3: Turbine power characteristics at zero pitch angle. 
Alternative to speed regulation, torque regulation can be applied in order to achieve MPPT for a WT. Considering (4.3) and (4.4), the optimum mechanical torque can be expressed by (4.7), provided that $T_{m}=\frac{P_{m}}{\omega_{m}}=\frac{1}{2} \rho A r C_{p} v_{w}^{2} / \lambda$.

$$
T_{m_{o p t}}=K_{o p t} \omega_{m}^{2}
$$

In (4.7), $K_{o p t}=\frac{\rho A}{2} C_{p_{\max }}\left(\frac{r}{\lambda_{\text {opt }}}\right)^{3}$.

As can be seen from (4.3), calculation of wind power requires knowledge of instantaneous wind speed. Wind speed is a stochastic variable signal and its behaviour is difficult to predict, especially over short-time period (i.e., seconds, minutes or few hours). Different Statistic wind speed models were summarized in [120]. One of commonly used wind speed models is the spectral density-based model, which describes the variation of wind speed as an overlapping of different frequency components. The basic model of the turbulent component, used in the Von-Karman power spectrum model [121] is given in (4.8).

$$
S(\omega)=\frac{0.475 \sigma^{2}\left(L / \bar{v}_{w}\right)}{\left[1+\left(\frac{\omega L}{\bar{v}_{w}}\right)^{2}\right]^{5 / 6}}
$$

In (4.8), $\omega$ is the circular frequency obtained by multiplying $\bar{v}_{\omega}$ with the spatial frequency $\Omega$ $(\mathrm{rad} / \mathrm{m}), \bar{v}_{w}$ the mean wind speed (typically, determined over 10-minute period), $\sigma$ the turbulence intensity, and $L$ the turbulence length scale in feet. For low-altitude model (altitude $<1000$ feet) [122],

$$
L=\frac{h}{(0.177+0.000823 h)^{1.2}}
$$

where $h$ is the height in feet at which the wind speed signal is of interest.

The turbulence intensity $\sigma$ is found from (4.10), where $v_{w_{20}}$ is the wind speed at 20 feet (6 meters). Typically, $v_{w_{20}}$ is $7.72 \mathrm{~m} / \mathrm{s}, 15.43 \mathrm{~m} / \mathrm{s}$, and $23.15 \mathrm{~m} / \mathrm{s}$ for light, moderate, and severe turbulence, respectively [122].

$$
\sigma=\frac{0.1 v_{w_{20}}}{(0.177+0.000823 h)^{0.4}}
$$


The amplitude $\left(A_{i}\right)$ of wind speed fluctuation (Amplitude of the $i^{\text {th }}$ harmonic), at discrete frequency of $f_{i}(i=1,2,3, \ldots M)$, where $M$ is the number of samples, is expressed by (4.11) [121].

$$
A_{i}\left(\omega_{i}\right)=\frac{2}{\pi} \sqrt{0.5\left[S\left(\omega_{i}\right)+S\left(\omega_{i+1}\right)\right]\left(\omega_{i+1}-\omega_{i}\right)}
$$

The instantaneous value of wind speed $v_{w}(t)$ can be described as a sum of average wind speed and fluctuation of the wind, as given by (4.12), where $\psi_{i}$ is a uniformly-distributed random phase angle in the domain $[\pi,-\pi]$.

$$
v_{W}(t)=\bar{v}_{w}+\sum_{i=1}^{M} A_{i} \cos \left(\omega_{i} t+\psi_{i}\right)
$$

Based on (4.8), a spectral density function is shown in Fig. 4.4(a). The figure is obtained for $M=55, \bar{v}_{\omega}=10 \mathrm{~m} / \mathrm{s}, v_{w_{20}}=7.72 \mathrm{~m} / \mathrm{s}$, and $h=18 \mathrm{~m}$. The low-pass filtered fluctuation of wind speed is shown in Fig. 4.4(b).
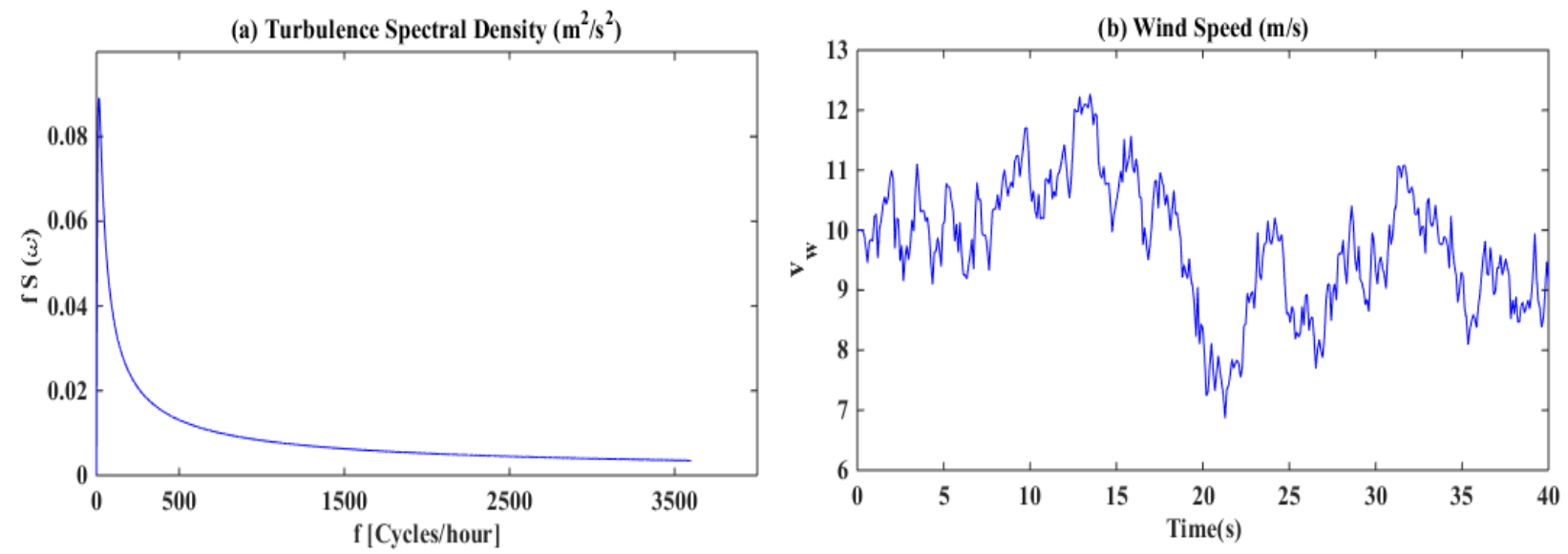

Fig. 4.4: Wind speed model based on Von-Karman's method.

\subsection{Drive Train System}

The drive train is essentially represented by a three-mass model which is composed of three masses accounting for the turbine's rotor, the gearbox and the generator [123]. The masses are linked by two shafts. The moments of inertia of the gearbox and the shafts are assumed to have small influence on the system behaviour compared to moment of inertia of the turbine rotor and generator. As a result, the three-mass model can be reduced to a two-mass model, shown in Fig. 4.5. It consists of two masses linked by an equivalent shaft. In this model, only the gear ratio $\left(n_{\text {gear }}\right)$ of the gearbox is considered. In Fig. $4.5, T_{m}, J_{m}$ and $\omega_{m}$ are the turbine torque in 
$N \cdot m$, moment of inertia in $\mathrm{kg} \cdot \mathrm{m}^{2}$ and angular speed in $\mathrm{rad} / \mathrm{s}$, respectively, $T_{e}, J_{g}$ and $\omega_{g}$ the generator torque in $N \cdot m$, moment of inertia in $\mathrm{kg} \cdot \mathrm{m}^{2}$ and angular speed in $\mathrm{rad} / \mathrm{s}$, respectively, $K_{s e}$ and $D_{s e}$ the shaft equivalent stiffness in $N \cdot \mathrm{m} / \mathrm{rad}$ and damping factor in $N \cdot \mathrm{m} / \mathrm{rad} / \mathrm{s}$, respectively, and $\theta_{m}$ and $\theta_{g}$ the turbine and generator shaft angles in rad, respectively.

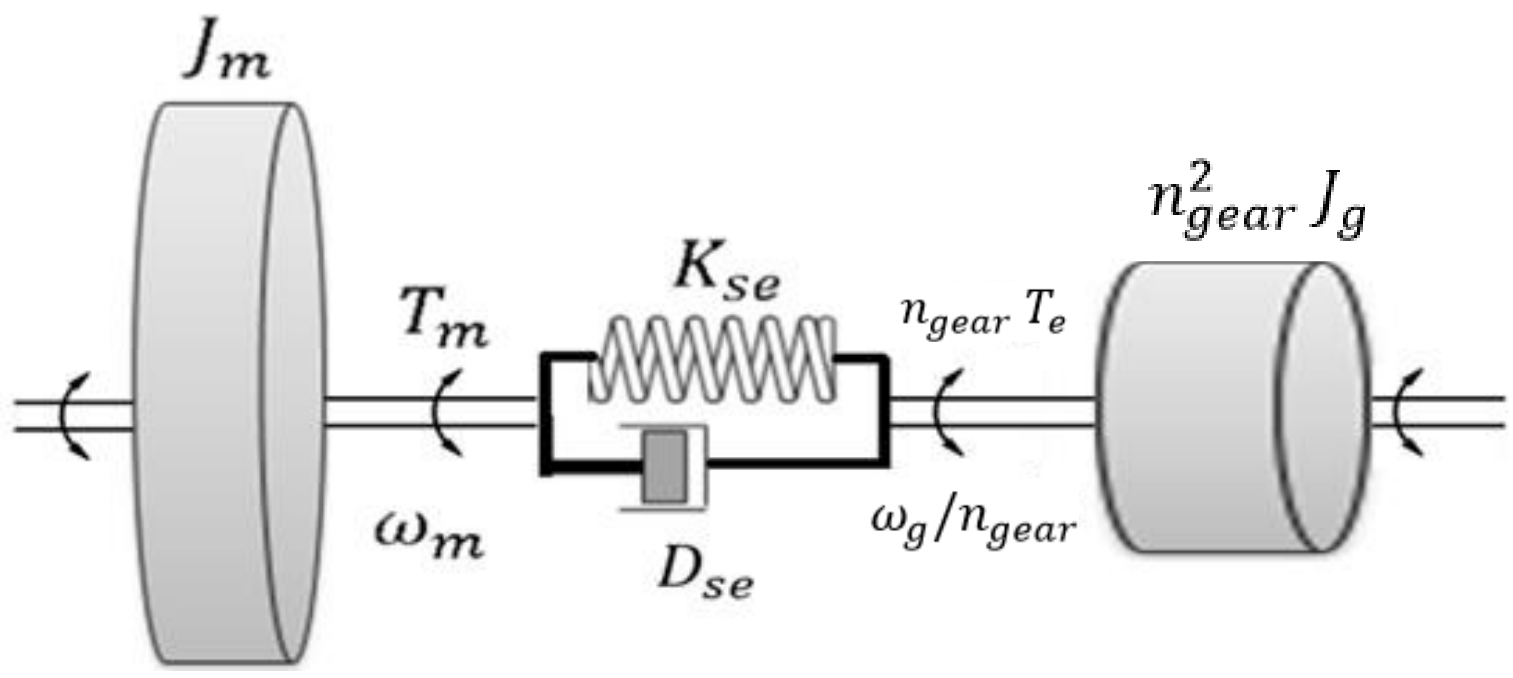

Fig. 4.5: Two mass model of turbine's drive train on Turbine side.

The dynamics of the drive train can be described by the following differential equations [123], where $\delta \theta=\theta_{m}-\theta_{g} / n_{\text {gear }}$ and $\delta \omega=\omega_{m}-\omega_{g} / n_{\text {gear }}$.

$$
\begin{gathered}
\frac{d}{d t} \omega_{m}=\frac{1}{J_{m}}\left[T_{m}-D_{\text {se }} \delta \omega-K_{s e} \delta \theta\right] \\
\frac{d}{d t} \omega_{g}=\frac{1}{J_{g}}\left[\frac{1}{n_{\text {gear }}} D_{\text {se }} \delta \omega+\frac{1}{n_{\text {gear }}} K_{\text {se }} \delta \theta-T_{e}\right] \\
\frac{d(\delta \theta)}{d t}=\delta \omega
\end{gathered}
$$

\subsection{Self-Excited Induction Machine}

Induction machine operates based on induction or transformer action between the stator and the rotor conductors. An electromagnetic torque is produced due to the interaction between stator and rotor magnetic fields. The difference between the rotor speed and the speed of 
rotating magnetic field of stator (synchronous speed), normalized to synchronous speed, defines slip (s) as:

$$
\mathrm{S}=\frac{\omega_{s}-\omega_{g}}{\omega_{s}}=\frac{\omega_{e}-\omega_{r}}{\omega_{e}}
$$

where $\omega_{s}$ is the synchronous angular speed in $\mathrm{rad} / \mathrm{s}, \omega_{e}$ the stator electrical angular frequency in $\mathrm{rad} / \mathrm{s}\left(\omega_{e}=P \omega_{s}\right), P$ the number of pole pairs, $\omega_{g}$ the rotor shaft angular speed in $\mathrm{rad} / \mathrm{s}$, and $\omega_{r}$ the rotor electrical angular speed in $\mathrm{rad} / \mathrm{s}\left(\omega_{r}=P \omega_{g}\right)$.

An induction generator needs to be continuously excited by a source of reactive power in order to generate voltage and supply active power. In grid-connected wind turbines, this reactive power is typically supplied by the grid. In standalone wind turbines, it can be supplied by either a power electronic converter or an external source of reactive power, such as switched capacitors. In this case, the generator is called self-excited induction generator (SEIG). In SEIG, the machine should operate in saturation region at the intersection point between the magnetization curve of the machine and the impedance of the excitation capacitor [124]. Either initial capacitor voltage or machine's residual magnetism is required for the flux to build up and the machine to operate as a generator. A combination of machine's parameters, shaft speed, excitation level, and loading condition determines the generated voltage magnitude and frequency. The minimum capacitor needed to generate the rated voltage at rated speed and no load condition is obtained by (4.17) [29] where $L_{m_{\text {unsaturated }}}$ is the magnetizing inductance before saturation.

$$
C_{\min }=\frac{1}{\omega_{r}^{2} L_{m} \text { unsaturated }}
$$

Amongst various models developed to analyze the transient and steady-state performances of self-excited induction machines [125], the well-known $d q$ model, shown at no load in Fig. 4.6, is used in this work. In the figure, $R_{s}$ is the stator resistance, $L_{l s}$ the stator leakage inductance, $L_{m}$ the magnetizing inductance, $R_{r}^{\prime}$ the rotor resistance refereed to the stator, $L_{l r}^{\prime}$ the rotor leakage inductance refereed to the stator, $C_{g}$ the excitation capacitance, $i_{d s}$ and $i_{q s}$ the $d$-axis and $q$-axis stator currents, respectively, $i_{m}$ the magnetizing current, $i_{d r}^{\prime}$ and $i_{q r}^{\prime}$ the $d$-axis and $q$-axis rotor currents referred to the stator, respectively, $v_{c_{g d}}$ and $v_{c_{g q}}$ the $d$-axis and $q$-axis voltage of the excitation capacitor, respectively, $\varphi_{d s}$ and $\varphi_{q s}$ the $d$-axis and $q$-axis 
stator flux, respectively, $\varphi_{d r}^{\prime}$ and $\varphi_{q r}^{\prime}$ the $d$-axis and $q$-axis rotor flux referred to the stator, respectively, and $p$ the derivative operator $(d / d t)$.

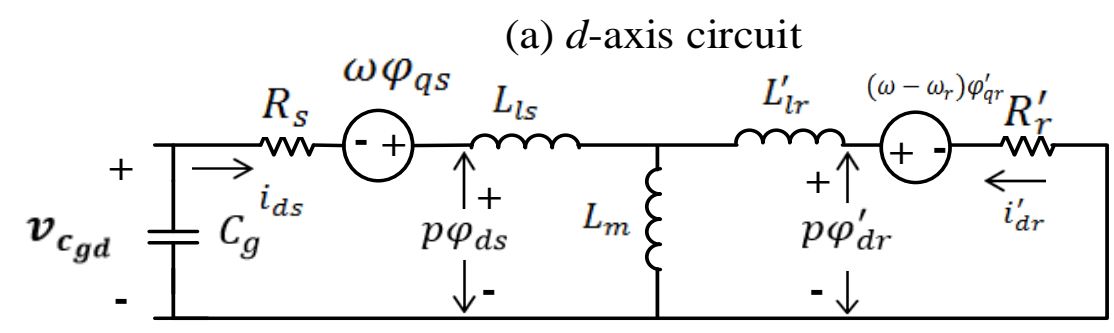

(b) $q$-axis circuit

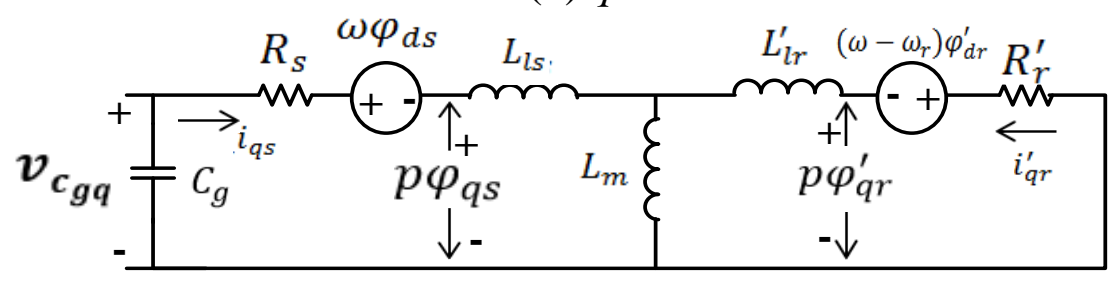

Fig. 4.6: $D q$ model of Self-excited squirrel cage induction machine.

The mathematical model of SEIG in an arbitrary $d q$ reference frame is described by the set of differential equations (4.18) - (4.21). All electrical variables and parameters indicated by the prime signs are referred to the stator.

$$
\begin{gathered}
v_{c_{g d}}=R_{s} i_{d s}+\frac{d \varphi_{d s}}{d t}-\omega \varphi_{q s} \\
v_{c_{g q}}=R_{s} i_{q s}+\frac{d \varphi_{q s}}{d t}+\omega \varphi_{d s} \\
0=R_{r}^{\prime} i_{d r}^{\prime}+\frac{d \varphi_{d r}^{\prime}}{d t}-\left(\omega-\omega_{r}\right) \varphi_{q r}^{\prime} \\
0=R_{r}^{\prime} i_{q r}^{\prime}+\frac{d \varphi_{q r}^{\prime}}{d t}+\left(\omega-\omega_{r}\right) \varphi_{d r}^{\prime}
\end{gathered}
$$

Equations (4.22) - (4.25) give the air gap flux linkages, with $L_{s}=L_{m}+L_{l s}$ and $L_{r}^{\prime}=L_{m}+L_{l r}^{\prime}$

$$
\begin{gathered}
\varphi_{q s}=L_{s} i_{q s}+L_{m} i_{q r}^{\prime} \\
\varphi_{d s}=L_{s} i_{d s}+L_{m} i_{d r}^{\prime} \\
\varphi_{q r}^{\prime}=L_{r}^{\prime} i_{q r}^{\prime}+L_{m} i_{q s}
\end{gathered}
$$




$$
\varphi_{d r}^{\prime}=L_{r}^{\prime} i_{d r}^{\prime}+L_{m} i_{d s}
$$

Equations (4.18)-(4.21) can be adapted for stationary, rotor and stator angular frequency reference frames, by substituting $0, \omega_{r}$, and $\omega_{e}$, respectively, for $\omega$. The $d q$ equations in stator reference frame (i.e., $\omega=\omega_{e}$ ) are given by (4.26), where $k_{s}=L_{m}^{2}-L_{s} L_{r}^{\prime}$.

$\frac{d}{d t}\left[\begin{array}{c}i_{q s} \\ i_{d s} \\ i_{q r}^{\prime} \\ i_{d r}^{\prime}\end{array}\right]=\frac{1}{k_{s}}$

$\left[\begin{array}{cccc}R_{s} L_{r}^{\prime} & \omega_{e}\left(L_{s} L_{r}^{\prime}-L_{m}^{2}\right)+\omega_{r} L_{m}^{2} & -R_{r}^{\prime} L_{m} & \omega_{r} L_{m} L_{r}^{\prime} \\ \omega_{e}\left(L_{m}^{2}-L_{s} L_{r}^{\prime}\right)-\omega_{r} L_{m}^{2} & R_{s} L_{r}^{\prime} & -\omega_{r} L_{m} L_{r}^{\prime} & -R_{r}^{\prime} L_{m} \\ -R_{s} L_{m} & -\omega_{r} L_{m} L_{s} & R_{r}^{\prime} L_{s} & \omega_{e}\left(L_{s} L_{r}^{\prime}-L_{m}^{2}\right)-L_{s} L_{r}^{\prime} \omega_{r} \\ \omega_{r} L_{m} L_{s} & -R_{s} L_{m} & \omega_{e}\left(L_{m}^{2}-L_{s} L_{r}^{\prime}\right)+L_{s} L_{r}^{\prime} \omega_{r} & R_{r}^{\prime} L_{s}\end{array}\right]\left[\begin{array}{l}i_{q s} \\ i_{d s} \\ i_{q r}^{\prime} \\ i_{d r}^{\prime}\end{array}\right]$ $+\frac{1}{k_{s}}\left[\begin{array}{cccc}-L_{r}^{\prime} & 0 & L_{m} & 0 \\ 0 & -L_{r}^{\prime} & 0 & L_{m} \\ L_{m} & 0 & -L_{s} & 0 \\ 0 & L_{m} & 0 & -L_{s}\end{array}\right]\left[\begin{array}{c}v_{c_{g q}} \\ v_{c_{g d}} \\ 0 \\ 0\end{array}\right]$

The reactive power, required to excite the machine, is provided by a star-connected capacitor bank. The capacitor $d q$ currents are given by (4.27) and (4.28).

$$
\begin{aligned}
& i_{c_{g d}}=C_{g} \frac{d}{d t} v_{c_{g d}}-C_{g} \omega_{e} v_{c_{g q}} \\
& i_{c_{g q}}=C_{g} \frac{d}{d t} v_{c_{g q}}+C_{g} \omega_{e} v_{c_{g d}}
\end{aligned}
$$

The electromagnetic torque $\left(T_{e}\right)$ generated by SCIG can be obtained by the relations given in (4.29).

$$
T_{e}=\left\{\begin{array}{l}
\frac{3 P}{2}\left(i_{q s} \varphi_{d s}-i_{d s} \varphi_{q s}\right) \\
\frac{3 P L_{m}}{2}\left(i_{q s} i_{d r}^{\prime}-i_{d s} i_{q r}^{\prime}\right) \\
\frac{3 P L_{m}}{2 L_{r}}\left(i_{q s} \varphi_{d r}^{\prime}-i_{d s} \varphi_{q r}^{\prime}\right)
\end{array}\right.
$$




\subsection{Three-Phase Diode Bridge with DC-Side Capacitive Filter}

In the proposed WECS, shown in Fig. 3.12, the SEIG generates a variable ac voltage which is converted into de voltage through a three-phase diode bridge rectifier. The rectifier's output voltage is smoothed by a capacitor of finite capacitance. Therefore, the dc-side voltage is not ripple free. The output dc current of the rectifier $\left(i_{d c r}\right)$ is boosted by a buck converter. Therefore, $i_{d c r}$ alternates between zero and $i_{d c h}$ depending on the state of the buck switch $\left(S_{\text {buck }}\right)$. In other words, the dc side of the bridge rectifier is equivalent to a current source when the buck switch, $S_{b u c k}$, is on, and an open circuit when $S_{b u c k}$ is off. Equivalent circuit diagram of the topology is shown in Fig. 4.7.

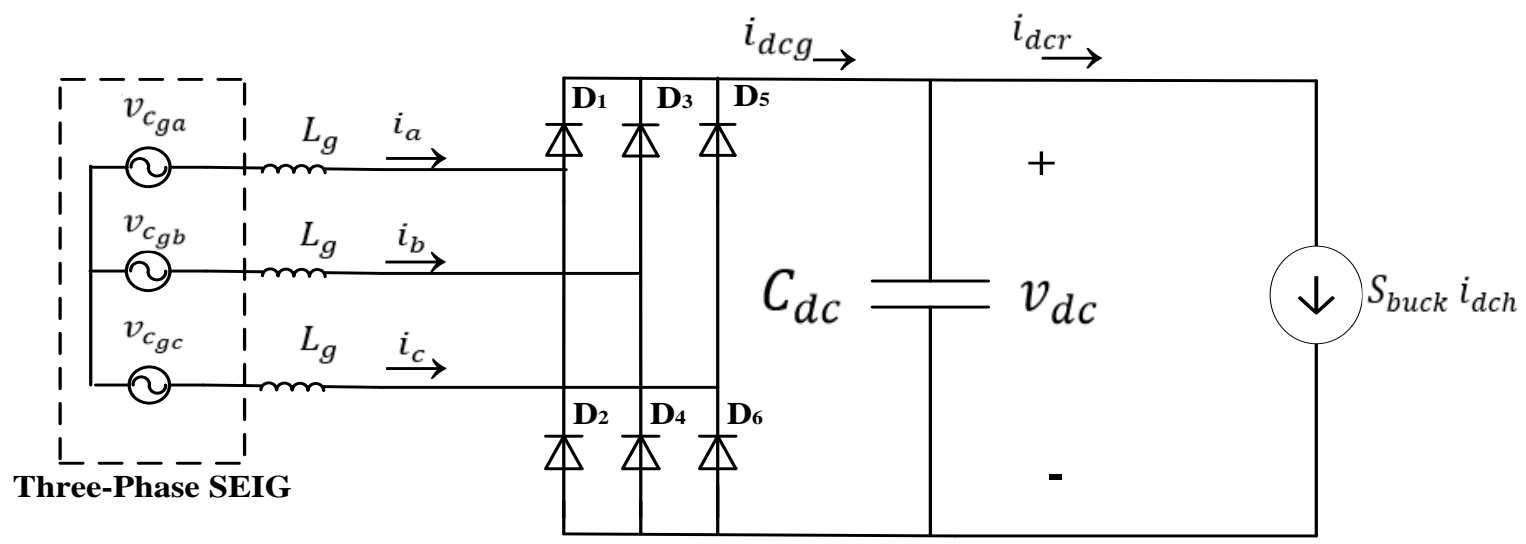

Fig. 4.7: Three-phase diode bridge rectifier with a variable dc current.

In Fig. 4.7, the SEIG is represented by a three-phase ac voltage supply, with the following time functions:

$$
\left[\begin{array}{l}
v_{c_{g a}} \\
v_{c_{g b}} \\
v_{c_{g c}}
\end{array}\right]=\left[\begin{array}{c}
\widehat{V}_{c_{g}} \sin \left(\omega_{e} t\right) \\
\widehat{V}_{c_{g}} \sin \left(\omega_{e} t-2 \pi / 3\right) \\
\widehat{V}_{c_{g}} \sin \left(\omega_{e} t+2 \pi / 3\right)
\end{array}\right]
$$

Depending on the values of the ac-side inductor $L_{g}$ and dc-side capacitor $C_{d c}$, and/or loading conditions, the topology shown in Fig. 4.7 can operate in one of the two different modes: Discontinues Conduction Mode (DCM) and Continuous Conduction Mode (CCM) [126].

In order to derive the average model of diode bridge rectifier in Fig. 4.7, the following assumptions are made: 
1. The self-excited IG generates three-phase balanced sinusoidal voltages.

2. The series inductance $L_{g}$ is negligible. This assumption is justifiable for low-power induction generator-based turbines. $L_{g}$ represents the cable and generator-side L filter. Real data show that the stator inductance in low-voltage, low-power induction generator $(<$ $100 \mathrm{~kW}$ ), is relatively high (i.e., $0.1-0.25 \mathrm{pu}$ ) [127],[128] compared with those in Mega-Watt IGs. The combination of the stator inductance and the excitation capacitance can provide acceptable harmonic attenuation to the generator current. Therefore, no external L filter is required to be added in the generator side and hence some cost is saved. Thus, $L_{g}$ is mainly attributed to the cable inductance. Reactance of low-voltage cables (up to $1 \mathrm{kV}$ ) is in the range of $0.1-0.75 \Omega / \mathrm{mile}$ at $60 \mathrm{~Hz}$ (i.e., $0.27-2 \mathrm{mH} / \mathrm{mile}$ ) [129]. In a wind turbine, the generator and its converter are placed in the nacelle and hence the length of cable connection between the generator and the diode-bridge is very short (around 1-2 meters) and cable inductance is too small $(<2.5 \mu \mathrm{H})$ and can be neglected.

3. The dc-side filter capacitor is large (0.3-1.5 pu), but not significant enough to force the bridge into DCM operation. When the $S_{b u c k}$ is turned off, the diode bridge output current $\left(i_{d c g}\right)$ flows through $C_{d c}$. Hence, $i_{d c g}$ never falls to zero. In other words, the bridge is always operating at CCM with instantaneous commutation (i.e., the commutation angle is very small and hence approximated to zero).

4. The voltage across the dc-side capacitor $\left(v_{d c}\right)$ has very slow dynamics and hence $v_{d c}$ may be considered constant with respect to state variables that vary under the influence of high switching frequency of the converters.

\section{All harmonics are neglected.}

6. As noticed from Fig. 3.12, the reduced H-bridge terminals are connected in series with the dc link inductor during non-dumping periods (i.e., $i_{d c h}=i_{d c}$ ). Thus, the average value of the rectifier output current $i_{d c g}$ is equal to the average value of $i_{d c r}$ which depends on the duty ratio of buck converter $d_{b}$, i.e., $i_{d c g}=i_{d c r}=d_{b} i_{d c h}=d_{b} i_{d c}$. For certain values of $d_{b}$ and $i_{d c}$, the average output current of the rectifier is constant. 
Based on the assumptions made above, the circuit of Fig. 4.7 can be approximated by that shown in Fig. 4.8.

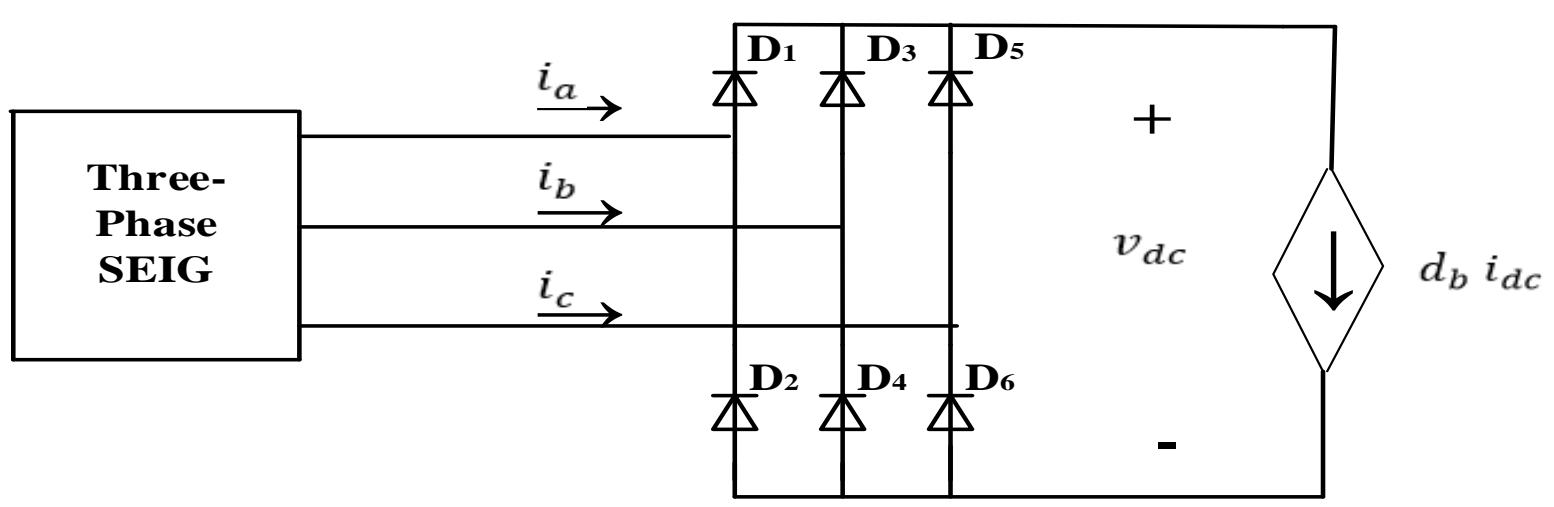

Fig. 4.8: Three-phase diode bridge rectifier with a constant dc current.

In Fig. 4.8, the average output voltage is found from (4.31), where $\widehat{V}_{c_{g}}$ is the peak of generator phase voltage.

$$
v_{d c}=\frac{2}{2 \pi / 6} \int_{0}^{\pi / 6} \sqrt{3} \widehat{V}_{c_{g}} \cos \omega t d\left(\omega_{e} t\right)=\frac{3 \sqrt{3}}{\pi} \widehat{V}_{c_{g}}
$$

The rms value of the fundamental component of the ac line current can be found by (4.32) [130].

$$
I_{s_{1 r m s}}=\frac{\sqrt{6}}{\pi} d_{b} i_{d c}
$$

From (4.31) and (4.32), diode bridge rectifier is represented by its average output, as shown in Fig. 4.9. In the figure, the supply voltages and ac-side currents are approximated by their fundamental components, assuming negligible harmonic contents. $\theta_{i}$ is phase angle between fundamental component of current and supply voltage. In the switching model, the fundamental current is slightly leading the supply voltage due to dc-side capacitor effect. Hence, a small part of the reactive power absorbed by the induction generator is supported by the $C_{d c}$. For the average model, however, the effect of $C_{d c}$ is neglected and the reactive power required for generator excitation is completely supplied by the capacitor bank. Under this assumption, the fundamental component of line current is in phase with the supply voltage (i.e., $\left.\theta_{i}=0\right)$. 
In Fig. 4.9, the effect of load side on the dc average voltage $\left(v_{d c}\right)$ is neglected. This assumption is valid as $v_{d c}$ is mainly dependent on the ac-side applied voltage.

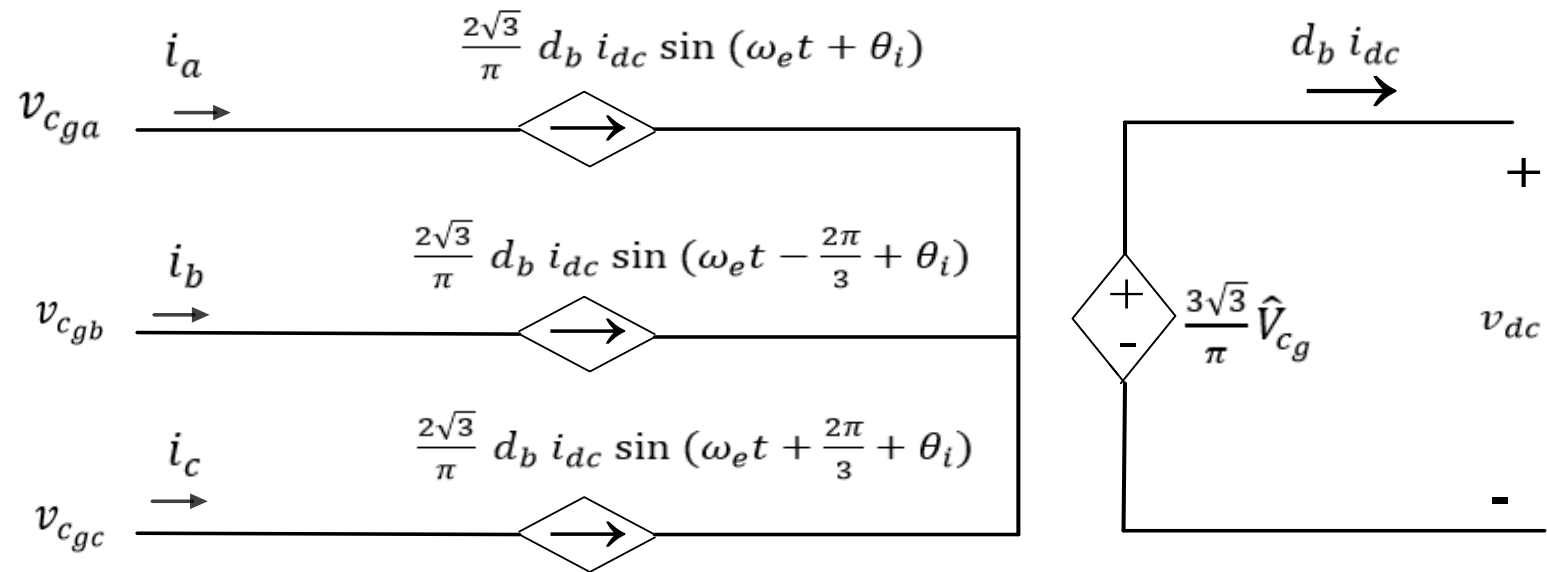

Fig. 4.9: Averaged model of CCM diode bridge rectifier with instantaneous commutation.

By transforming the three-phase supply voltages and currents into $d q$ frame rotating at angular electrical speed of the generator, $\omega_{e}$, the abc-based average model, shown in Fig. 4.9, is redrawn in $d q$ frame in Fig. 4.10. Assuming that the $d$-axis is initially aligned with the stator terminal phase voltage and diode bridge rectifier input current is in phase with that voltage, $v_{c_{g d}}=\widehat{V}_{c_{g}}, v_{c_{g q}}=0, i_{q}=0$. Thus, from (4.31) and (4.32), $v_{d c}=\frac{3 \sqrt{3}}{\pi} v_{c_{g d}}$ and $i_{d}=\frac{2 \sqrt{3}}{\pi} d_{b} i_{d c}$.
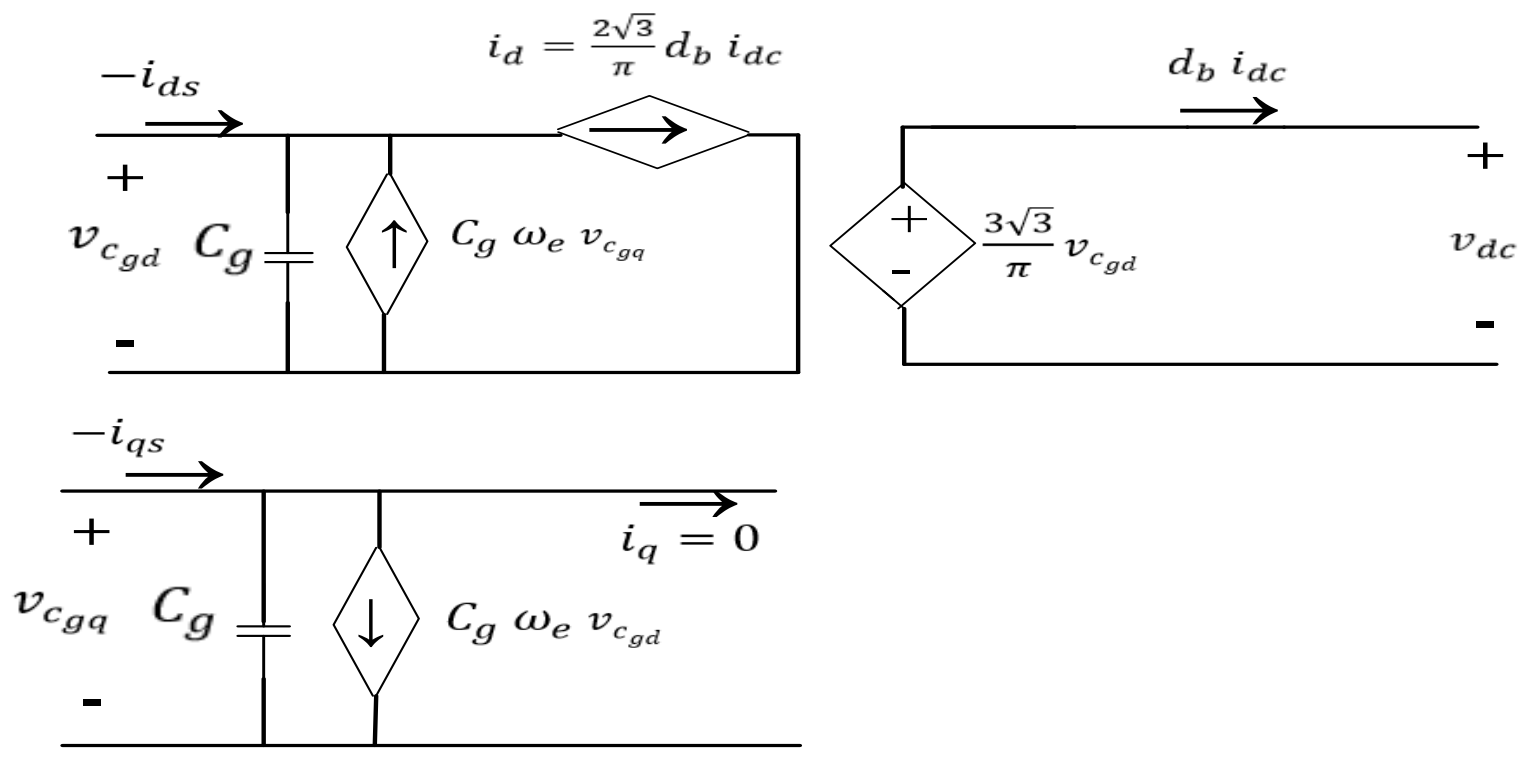

Fig. 4.10: $D q$ average model of diode bridge rectifier supplied by SEIG. 
Applying KCL to Fig. 4.10, the dynamics of capacitor voltage is described by (4.33) and (4.34).

$$
\begin{gathered}
\frac{d}{d t} v_{c g d}=-\frac{1}{c_{g}} i_{d s}-\frac{1}{c_{g}} \frac{2 \sqrt{3}}{\pi} d_{b} i_{d c}+\omega_{e} v_{c g q} \\
\frac{d}{d t} v_{c_{g q}}=-\frac{1}{c_{g}} i_{q s}-\omega_{e} v_{c_{g d}}
\end{gathered}
$$

\subsection{DC/DC Buck Converter}

In the proposed system (Fig. 3.12), the dc/dc buck converter is placed between the diode bridge rectifier and the PWM-CSI. As mentioned in the previous section, except during dumping periods, the converter output current $i_{d c h}$ is equal to the dc-link current $i_{d c}$ which is kept continuous and regulated via the large dc-link reactor and the control implemented by the $\mathrm{H}$-bridge interfacing the storage battery bank. Hence, the buck converter is operating in Continuous Conduction Mode (CCM). The buck converter employed in the proposed system is shown in Fig. 4.11.

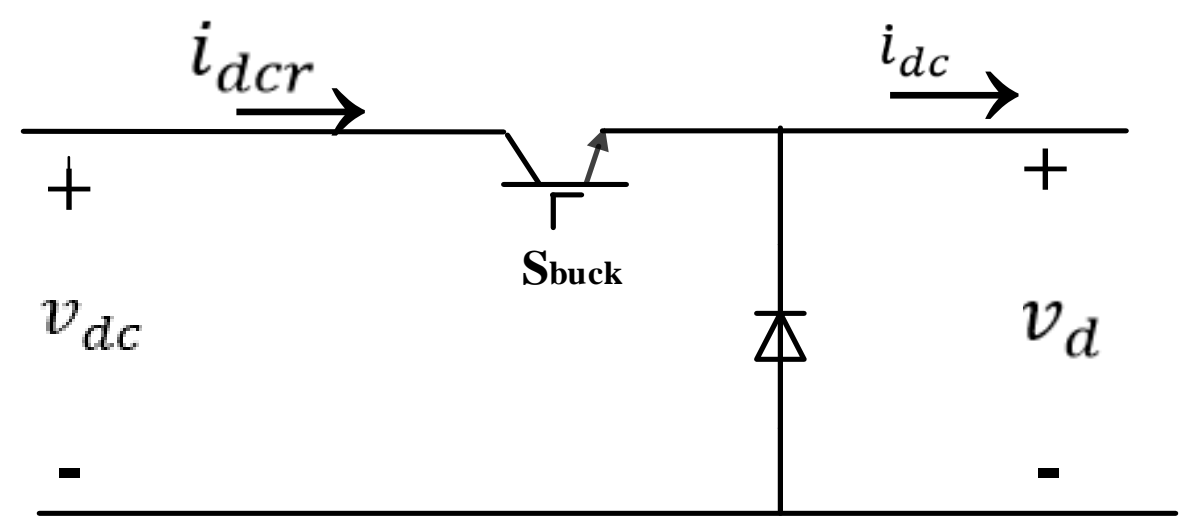

Fig. 4.11: Buck converter circuit.

In Fig. 4.11, the average values of $i_{d c r}$ and $i_{d c}$ are related through the duty cycle of the buck switch, $d_{b}$.

$$
i_{d c r}=d_{b} i_{d c}
$$

The converter is boosting the current and hence bucking the voltage. Thus,

$$
v_{d}=d_{b} v_{d c}
$$

Based on (4.35) and (4.36), the equivalent circuit of the converter is shown in Fig. 4.12. 


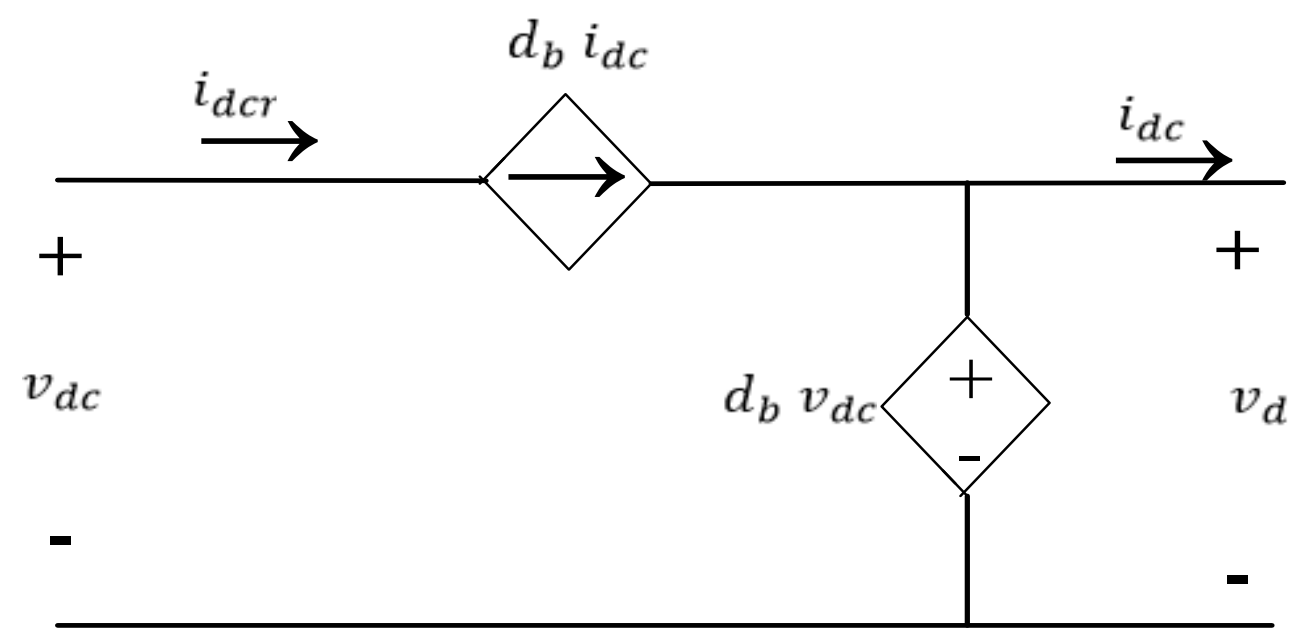

Fig. 4.12: Large-signal nonlinear averaged model of CCM buck converter.

\subsection{Lead Acid Battery}

Fig. 4.13 shows one of the common models of lead-acid batteries reported in the literature [131],[132]. As shown in the figure, the voltage-current characteristics of a battery is modelled by a controlled voltage source $E_{o c}$ and series resistance $R_{\text {series }}$. As given in (4.37) and (4.38) [132], $E_{o c}$ and $R_{\text {series }}$ are functions of battery's state of charge SoC.

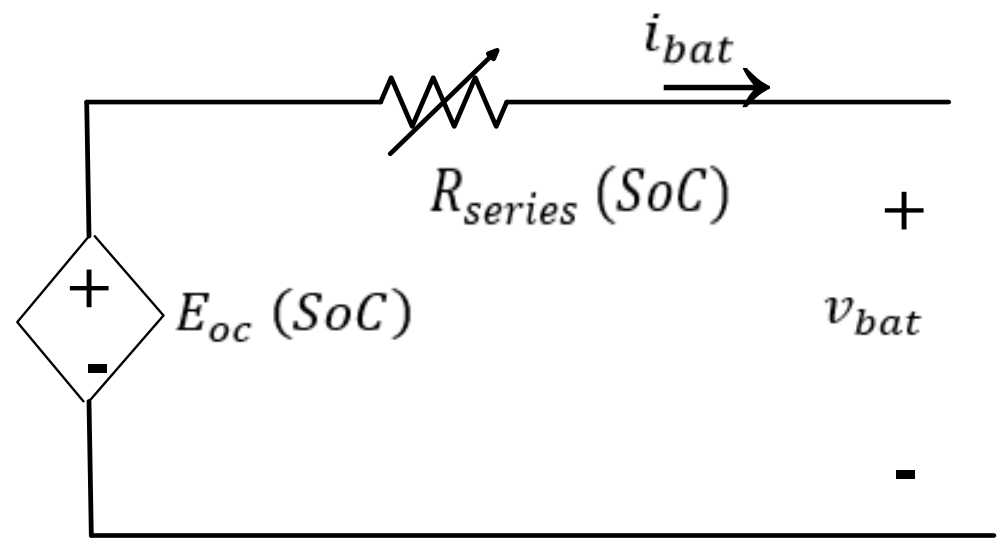

Fig. 4.13: Electrical model of a battery cell.

$$
\begin{gathered}
E_{o c}=E_{o}-K_{E}(1-S o C) \\
R_{\text {series }}=R_{o}\left(1+A_{0}(1-S o C)\right)
\end{gathered}
$$

where 


$$
\begin{gathered}
S o C=1-\frac{Q_{e}}{Q_{o}} \\
Q_{e}=\int_{0}^{t} i_{b a t} d t
\end{gathered}
$$

In (4.37)-(4.40), $E_{o}$ is the open-circuit voltage at $100 \%$ SoC, $K_{E}$ and $A_{o}$ constants, $R_{o}$ the series resistance at $100 \% \mathrm{SoC}, Q_{o}$ and $Q_{e}$ the battery rated Ampere-hour (Ah) capacity and extracted $\mathrm{Ah}$, respectively, and $i_{\text {bat }}$ the battery current. It is assumed that the battery packs are kept in a temperature-controlled environment and hence the effect of temperature variation is not modelled. From Fig. 4.13, the battery terminal voltage is given by (4.41), where a positive $i_{b a t}$ implies battery is being discharged.

$$
v_{\text {bat }}=E_{\text {oc }}-R_{\text {series }} i_{\text {bat }}
$$

In the proposed WECS (Fig. 3.12), the reduced H-bridge terminals are connected in series with the dc link inductor. Thus, the battery-side current $i_{i n}$ is composed of pulses of magnitude $i_{d c}$ (discharging) or $-i_{d c}$ (charging), separated by zero-current periods (freewheeling). A lowpass L-C filter is used to smooth out the battery current (See Fig. 4.14). Design of LC filter is based on a specified cut-off frequency and damping ratio, as explained in Appendix $B$.

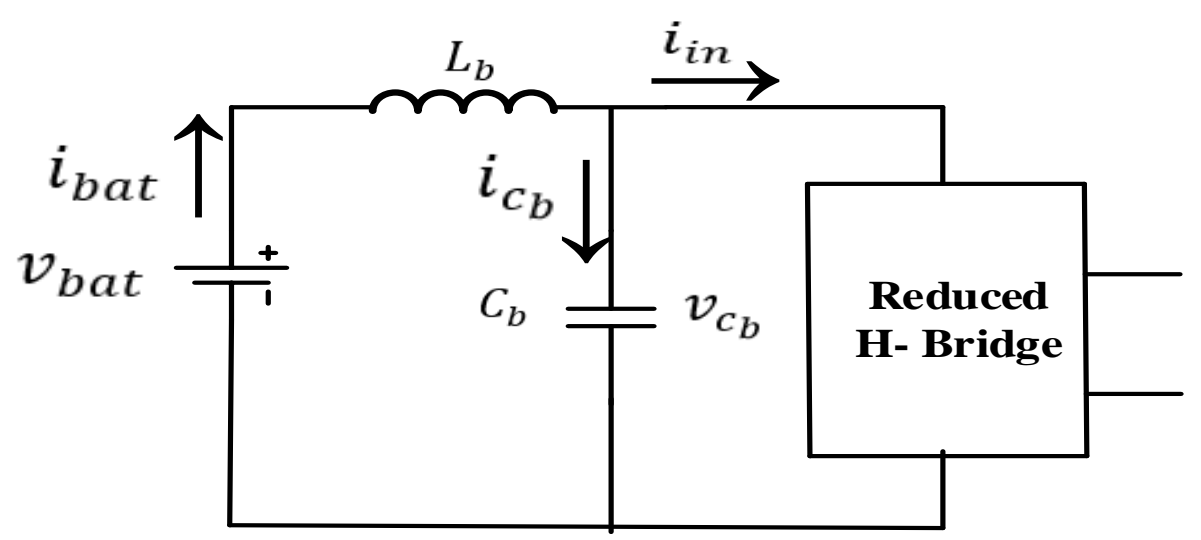

Fig. 4.14: LC filter on battery side.

Based on Fig. 4.14, the dynamic of the battery-side filter is described by (4.42) and (4.43).

$$
\begin{gathered}
\frac{d}{d t} i_{b a t}=\frac{1}{L_{b}}\left(v_{b a t}-v_{c_{b}}\right) \\
\frac{d}{d t} v_{c_{b}}=\frac{1}{c_{b}}\left(i_{b a t}-i_{\text {in }}\right)
\end{gathered}
$$




\subsection{DC/DC Reduced H- Bridge Converter}

Fig. 4.15 shows the dc/dc H-bridge converter, with reduced number of switches and diodes, employed as the interface between the storage battery bank and the dc link. The terminal current of the H-bridge is equal to $i_{d c}$; hence, the load of the H-bridge can be represented by a dc current source.

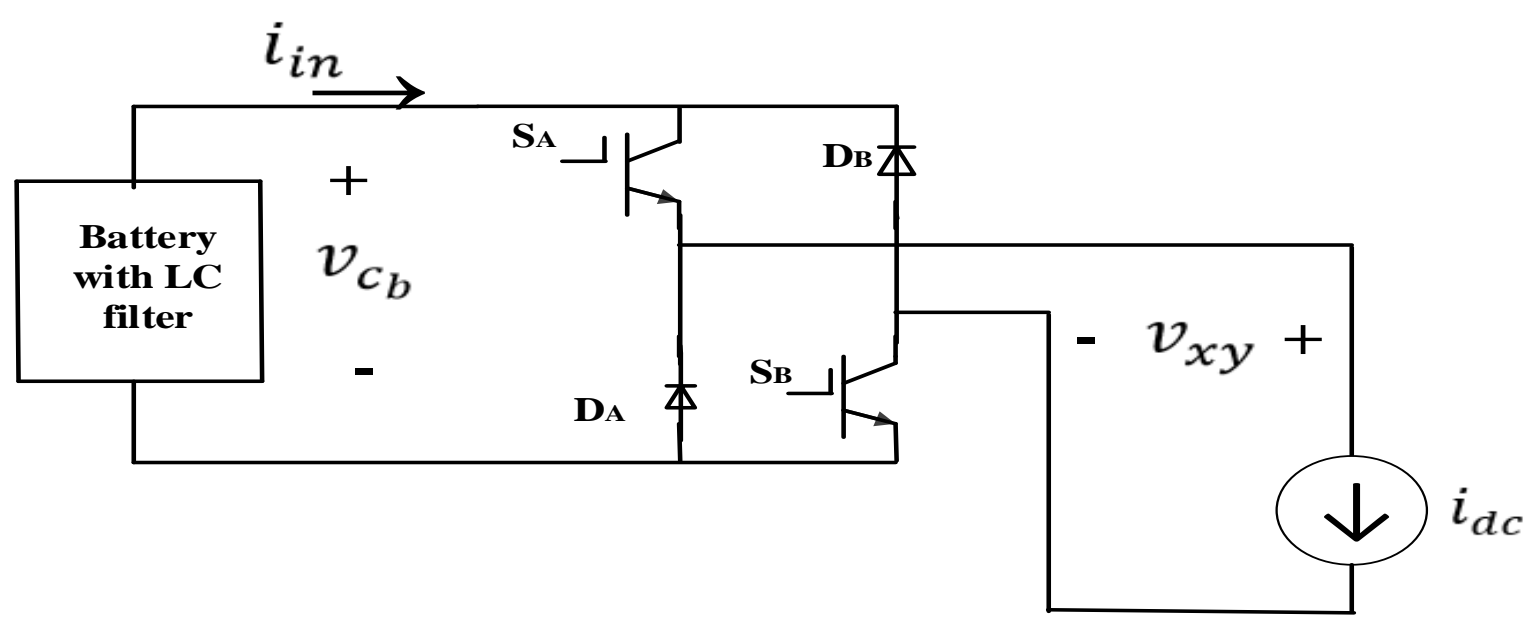

Fig. 4.15: Reduced H-bridge for storage integration.

The reduced H-bridge can be controlled by either PWM bipolar voltage or PWM unipolar voltage switching scheme. Unipolar voltage switching scheme provides frequency doubling effect, resulting in higher-quality waveforms. In this switching scheme, the two switches $\mathrm{S}_{A}$ and $S_{B}$ of the converter are controlled independently. The switch and diode in each leg can never conduct at the same time; hence, short circuiting of the dc source (i.e., the battery) is always avoided. Table 4.1 shows the possible output voltage values at different states of the two switches.

Table 4.1: Reduced H-bridge converter operating modes.

\begin{tabular}{ccccl}
\hline $\boldsymbol{S}_{\boldsymbol{A}}$ & $\boldsymbol{S}_{\boldsymbol{B}}$ & $\boldsymbol{v}_{\boldsymbol{x y}}$ & $\boldsymbol{i}_{\boldsymbol{i n}}$ & \multicolumn{1}{c}{ Mode } \\
\hline 0 & 0 & $-v_{c_{b}}$ & $-i_{d c}$ & Charging through $D_{B}$ and $D_{A}$ \\
0 & 1 & 0 & 0 & Freewheeling state through $S_{B}$ and $D_{A}$ \\
1 & 0 & 0 & 0 & Freewheeling state through $D_{B}$ and $S_{A}$ \\
1 & 1 & $v_{c_{b}}$ & $i_{d c}$ & Discharging through $S_{A}$ and $S_{B}$ \\
\hline
\end{tabular}


Let $d_{A}$ be the duty ratio of switch $S_{A}$. The average values of output voltage $v_{x y}$ and input current $i_{\text {in }}$ can be expressed by (4.44) and (4.45), respectively.

$$
\begin{gathered}
v_{x y}=\left(2 d_{A}-1\right) v_{c_{b}} \\
i_{\text {in }}=\left(2 d_{A}-1\right) i_{d c}
\end{gathered}
$$

Based on (4.44) and (4.45), the equivalent circuit diagram of the converter is shown in Fig. 4.16.

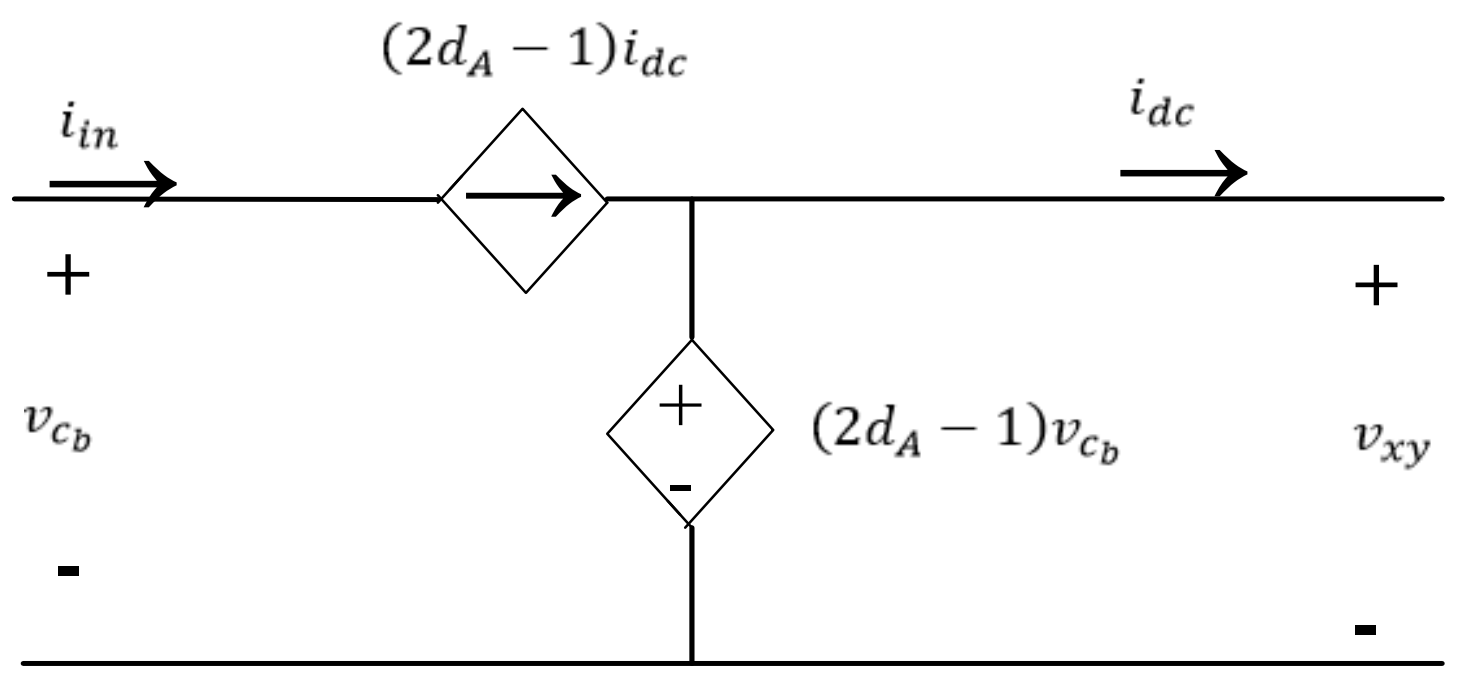

Fig. 4.16: Large-signal nonlinear averaged model of CCM Full-Bridge Converter.

Substituting (4.41) and (4.45) in (4.42) and (4.43), respectively, the dynamic of storage-side LC filter is described by the following differential equations.

$$
\begin{gathered}
\frac{d}{d t} i_{b a t}=\frac{1}{L_{b}}\left(E_{o}-K_{E}(1-S o C)-R_{o}\left(1+A_{0}(1-S o C)\right) i_{b a t}-v_{c_{b}}\right) \\
\frac{d}{d t} v_{c_{b}}=\frac{1}{c_{b}}\left(i_{b a t}-\left(2 d_{A}-1\right) i_{d c}\right)
\end{gathered}
$$

From (4.39) and (4.40), the dynamic of SoC is described by

$$
\frac{d}{d t} S o C=-\frac{1}{Q_{o}} i_{b a t}
$$

The dynamic of SoC is very slow with respect to other states. Therefore, for stability analysis, carried out around an operating steady-state point, SoC is assumed constant. 


\subsection{Current Source Inverter}

In this section, the current source inverter is modelled under balanced and unbalanced load conditions.

In the proposed WECS, shown in Fig. 3.12, the inverter dc side is supplied by the power received from a wind turbine, augmented by a storage system. This combination can represent a dispatchable distributed energy resource unit, producing a controllable current (i.e., $i_{d c}$ ), and hence can be represented by a dc current source, as shown in Fig. 4.17. In the figure, the threephase current-sourced inverter is feeding a three-phase load. The relationship between the acside and dc-side voltages and currents of CSI are determined by the switching functions of the three legs of the inverter. The switching constraints and states in the operation of CSI are explained in Appendix C. The switching actions of the inverter generate high-frequency harmonics, which are significantly reduced by the $\mathrm{C}$-filter $\left(C_{i}\right)$, and hence can be neglected, as far as the fundamental components of output-side currents are concerned.

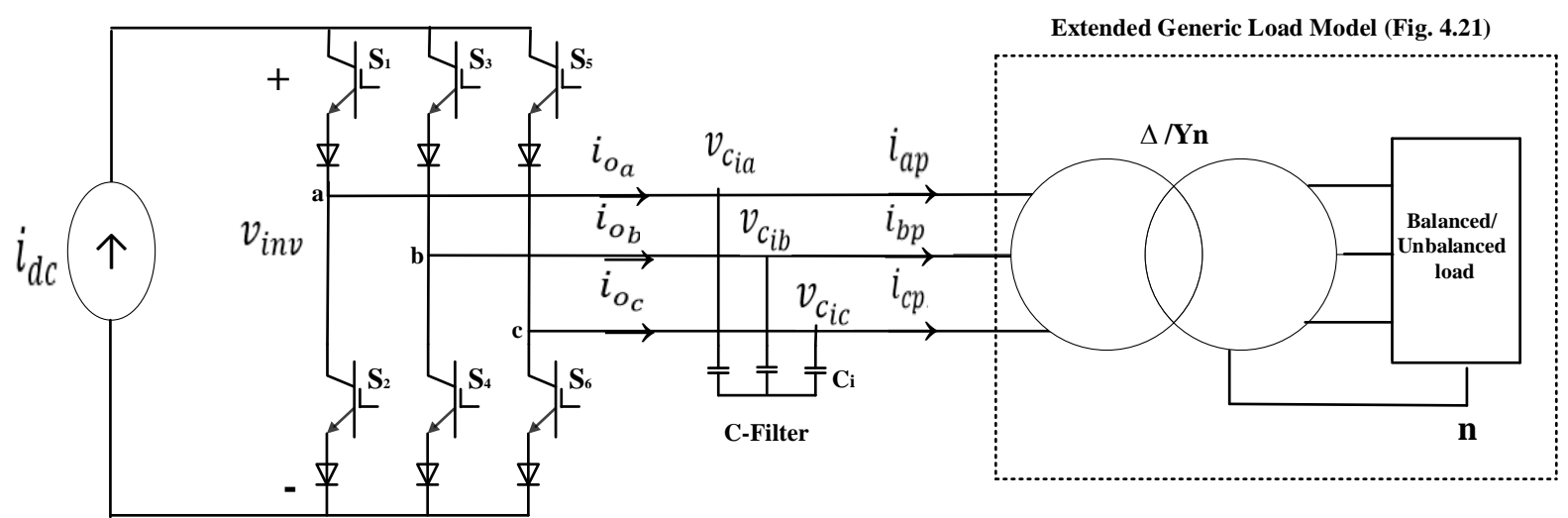

Fig. 4.17: Three-phase current-sourced inverter feeding a three-phase load.

\subsubsection{ABC-Frame Equations}

Under three-phase balanced load condition, the inverter supplies the load with three-phase balanced voltages and currents. Under a PWM scheme, the fundamental components of the acside currents are related to the dc-side current as in (4.49).

$$
\left[i_{o}\right]_{a b c}=G\left[m_{i}\right]_{a b c} i_{d c}
$$

In (4.49), 


$$
\left[m_{i}\right]_{a b c}=\left[\begin{array}{l}
m_{i a} \\
m_{i b} \\
m_{i c}
\end{array}\right]=\left[\begin{array}{c}
m_{i} \sin \left(\omega_{L} t-\alpha_{i}\right) \\
m_{i} \sin \left(\omega_{L} t-\alpha_{i}-2 \pi / 3\right) \\
m_{i} \sin \left(\omega_{L} t-\alpha_{i}+2 \pi / 3\right)
\end{array}\right]
$$

where $m_{i}$ and $\alpha_{i}$ are the modulation index $\left(0<m_{i}<1\right)$ and delay angle of the inverter, respectively, $G$ the ac gain of the corresponding PWM scheme ( $G=\frac{\sqrt{3}}{2}$ for sinusoidal PWM) and $\omega_{L}$ the fundamental frequency of the load-side voltage (i.e., $100 \pi$ or $120 \pi \mathrm{rad} / \mathrm{s}$ for $50 \mathrm{~Hz}$ or $60 \mathrm{~Hz}$, respectively). Ignoring the inverter losses, the power balance of the ac-side and dcside of the inverter gives:

$$
v_{i n v} i_{d c}=\left[i_{o}\right]_{a b c}^{T}\left[v_{c_{i}}\right]_{a b c}
$$

where $v_{i n v}$ is the average dc-input voltage and $\left[v_{c_{i}}\right]_{a b c}$ the three-phase capacitor voltages, defined as

$$
\left[v_{c_{i}}\right]_{a b c}=\left[\begin{array}{c}
\hat{V}_{c_{i}} \sin \left(\omega_{L} t+\theta_{v_{c}}\right) \\
\hat{V}_{c_{i}} \sin \left(\omega_{L} t+\theta_{v_{c}}-2 \pi / 3\right) \\
\widehat{V}_{c_{i_{c}}} \sin \left(\omega_{L} t+\theta_{v_{c}}+2 \pi / 3\right)
\end{array}\right]
$$

Substituting (4.49) into (4.51), yields

$$
v_{i n v}=G\left[m_{i}\right]_{a b c}^{T}\left[v_{c_{i}}\right]_{a b c}
$$

Applying KCL on the ac-side of the inverter, the dynamics of $\mathrm{C}$-filter is described by:

$$
\frac{d}{d t}\left[v_{c_{i}}\right]_{a b c}=\frac{1}{C_{i}}\left[i_{o}\right]_{a b c}-\frac{1}{C_{i}}\left[i_{p}\right]_{a b c}
$$

where $\left[i_{p}\right]_{a b c}$ is the transformer three-phase primary-side current. Based on (4.49) and (4.53), the equivalent circuit diagram of the inverter is shown in Fig. 4.18. 


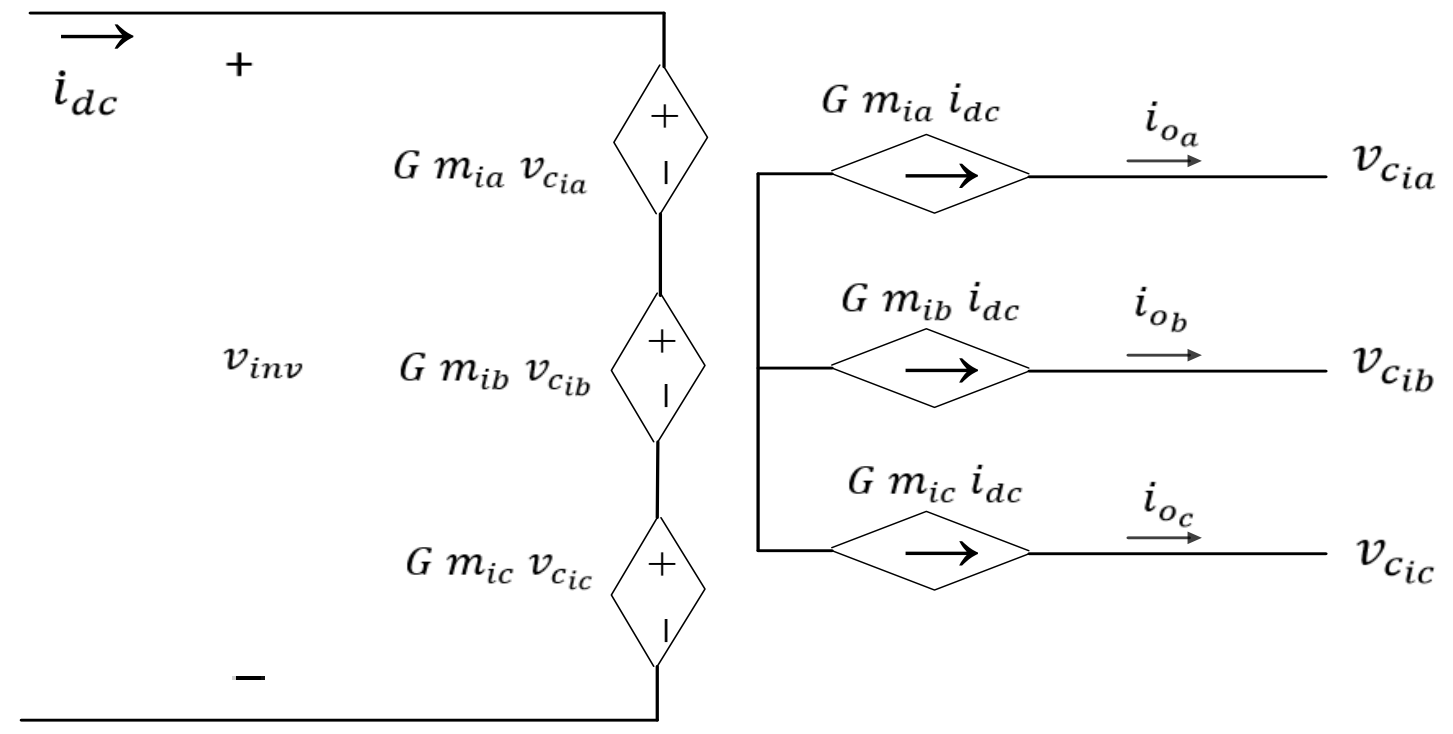

Fig. 4.18: Large-signal nonlinear averaged model of current source inverter.

Under three-phase unbalanced load condition, the three phase currents on the transformer secondary side are unbalanced, with positive (+ve), negative (-ve), and zero sequence (seq) components. Since the zero seq component is trapped in the $\Delta$ winding of the transformer, the primary-side three-phase currents (i.e., $i_{a p}, i_{b p}$ and $i_{c p}$ ) contain only +ve and -ve seq components. Now, every three-phase quantity in the ac-side of the inverter is divided into three-phase +ve and -ve seq symmetrical components, rotating at the fundamental frequency $\left(\omega_{L}\right)$, but with opposite phase sequence. In other words, $\left[m_{i}\right]_{a b c},\left[i_{o}\right]_{a b c},\left[v_{c_{i}}\right]_{a b c}$, and $\left[i_{p}\right]_{a b c}$ are divided into $\left[m_{i}^{+}\right]_{a b c}$ and $\left[m_{i}^{-}\right]_{a b c},\left[i_{o}^{+}\right]_{a b c}$ and $\left[i_{o}^{-}\right]_{a b c},\left[v_{c_{i}}^{+}\right]_{a b c}$ and $\left[v_{c_{i}}^{-}\right]_{a b c}$, and $\left[i_{p}^{+}\right]_{a b c}$ and $\left[i_{p}^{-}\right]_{a b c}$, respectively, where the +ve and -ve seq components can be defined by (4.55) and (4.56), respectively, by replacing the variable $x$ with the quantity under consideration.

$$
\begin{gathered}
{\left[x^{+}\right]_{a b c}=\left[\begin{array}{c}
\hat{X}^{+} \sin \left(\omega_{L} t-\theta_{x}^{+}\right) \\
\hat{X}^{+} \sin \left(\omega_{L} t-\theta_{x}^{+}-2 \pi / 3\right) \\
\hat{X}^{+} \sin \left(\omega_{L} t-\theta_{x}^{+}+2 \pi / 3\right)
\end{array}\right]} \\
{\left[x^{-}\right]_{a b c}=\left[\begin{array}{c}
\hat{X}^{-} \sin \left(\omega_{L} t-\theta_{x}^{-}\right) \\
\hat{X}^{-} \sin \left(\omega_{L} t-\theta_{x}^{-}+2 \pi / 3\right) \\
\hat{X}^{-} \sin \left(\omega_{L} t-\theta_{x}^{-}-2 \pi / 3\right)
\end{array}\right]}
\end{gathered}
$$




\subsubsection{DQ-Frame Equations}

For balanced load, where only +ve seq, three-phase voltages and currents exist, the control design of the CSI is based on a synchronously-rotating $d q$ frame. By transforming the abc voltages and currents into $d q$ frame rotating at $\omega_{L}$, the $d q$-axis equations of the inverter are obtained as

$$
\begin{gathered}
{\left[\begin{array}{c}
i_{o d}^{+} \\
i_{o q}^{+}
\end{array}\right]=G\left[\begin{array}{l}
m_{i d}^{+} \\
m_{i q}^{+}
\end{array}\right] i_{d c}} \\
v_{i n v}=1.5 G\left(m_{i d}^{+} v_{c i d}^{+}+m_{i q}^{+} v_{c i q}^{+}\right) \\
\frac{d}{d t}\left[\begin{array}{c}
v_{c i d}^{+} \\
v_{c i q}^{+}
\end{array}\right]=\frac{1}{C_{i}}\left[\begin{array}{c}
i_{o d}^{+} \\
i_{o q}^{+}
\end{array}\right]-\left[\begin{array}{cc}
0 & -\omega_{L} \\
\omega_{L} & 0
\end{array}\right]\left[\begin{array}{l}
v_{c i d}^{+} \\
v_{c i q}^{+}
\end{array}\right]-\frac{1}{C_{i}}\left[\begin{array}{l}
i_{p d}^{+} \\
i_{p q}^{+}
\end{array}\right]
\end{gathered}
$$

where $m_{i d}^{+}$and $m_{i q}^{+}$are the equivalent $d$ - and $q$-axis modulation indices of the inverter, respectively, $v_{c i d}^{+}$and $v_{c i q}^{+}$the $d$-and $q$-axis capacitor voltages, respectively, and $i_{p d}^{+}$and $i_{p q}^{+}$ the $d$ - and $q$-axis primary-side transformer currents, respectively.

For unbalanced load, the +ve and -ve seq abc components are transformed into $d q$ axis frames rotating at $\omega_{L}$ and $-\omega_{L}$, respectively. The resulting equations are given in (4.60)(4.62). Note that the sequence of a quantity is identified by the sign in its superscript.

$$
\begin{aligned}
& {\left[\begin{array}{l}
i_{o d}^{+} \\
i_{o q}^{+} \\
i_{o d}^{-} \\
i_{o q}^{-}
\end{array}\right]=G\left[\begin{array}{l}
m_{i d}^{+} \\
m_{i q}^{+} \\
m_{i d}^{-} \\
m_{i q}^{-}
\end{array}\right] i_{d c}} \\
& v_{i n v}=1.5 G\left(m_{i d}^{+} v_{c i d}^{+}+m_{i q}^{+} v_{c i q}^{+}+m_{i d}^{-} v_{c i d}^{-}+m_{i q}^{-} v_{c i q}^{-}\right) \\
& \frac{d}{d t}\left[\begin{array}{c}
v_{c i d}^{+} \\
v_{c i q}^{+} \\
v_{c i d}^{-} \\
v_{c i q}^{-}
\end{array}\right]=\frac{1}{c_{i}}\left[\begin{array}{l}
i_{o d}^{+} \\
i_{o q}^{+} \\
i_{o d}^{-} \\
i_{o q}^{-}
\end{array}\right]-\left[\begin{array}{cccc}
0 & -\omega_{L} & 0 & 0 \\
\omega_{L} & 0 & 0 & 0 \\
0 & 0 & 0 & \omega_{L} \\
0 & 0 & -\omega_{L} & 0
\end{array}\right]\left[\begin{array}{c}
v_{c i d}^{+} \\
v_{c i q}^{+} \\
v_{c i d}^{-} \\
v_{c i q}^{-}
\end{array}\right]-\frac{1}{C_{i}}\left[\begin{array}{c}
i_{p d}^{+} \\
i_{p q}^{+} \\
i_{p d}^{-} \\
i_{p q}^{-}
\end{array}\right]
\end{aligned}
$$

\subsection{Generic Load Model}

In a standalone energy system, non-linearity and variability (including frequent switching) of the load have significant adverse impact on the system's performance and may jeopardize the stability of voltage/frequency control. Therefore, load modeling is an essential part of 
stability analysis and controller design for a standalone system. The stability analysis carried out in [133] has been based on an RL load. Similarly, control schemes developed in [134] and [135] have assumed RL and RLC loads, respectively. A nonlinear load has been presented by a current source in [136]. The effect of induction motor loads on system's stability has been discussed in [137]. However, due to the diversity of residential loads, a generic load model, proposed by [138], is used in this thesis. The model is, in essence, capable of emulating actual loads of different transient and steady-state characteristics and is appropriate for simulation studies of stability and dynamic performance of a standalone system. The model has been successfully used to emulate RL and induction motor loads [138],[139]. However, the model has been mainly developed and validated for balanced load conditions. In the next subsections, the generic load model proposed in [138] will be described first. Then, the order of the model will be reduced by minimal realization. Finally, the reduced-order model will be extended to include unbalanced load condition.

\subsubsection{Generic Load Model Proposed in [138]}

The structure of the model is illustrated in Fig. 4.19. The load is modeled by three dependent current sources. The control signals for this model are obtained from $d q /$ abc transformation of the load $d$ - and $q$-axis currents, $i_{L_{d}}$ and $i_{L_{q}}$, that are dynamically determined based on load voltage $d q$ components, $v_{L_{d}}$ and $v_{L_{q}}$. As shown in Fig. 4.19 , the angle of transformation $\left(\theta_{L}\right)$ and angular velocity $\left(\omega_{L}\right)$ of the load voltage vector are obtained by implementing a phaselocked loop (PLL). In this model, the dynamics of the load is described by a set of state-space equations as:

$$
\begin{gathered}
\dot{X}_{L}=A_{L} X_{L}(t)+B_{L}\left[\begin{array}{l}
v_{L d}(t) \\
v_{L q}(t)
\end{array}\right] \\
Y_{L}(t)=C_{L} X_{L}(t)=\left[\begin{array}{l}
i_{L d}(t) \\
i_{L q}(t)
\end{array}\right]
\end{gathered}
$$

where $X_{L}$ is the vector of state variables, $Y_{L}$ the vector of outputs, $v_{L d}$ and $v_{L q}$ the $d$ - and $q$-axis components of load voltage (as inputs), $i_{L d}$ and $i_{L q}$ the $d$ - and $q$-axis components of load current (as outputs), and $A_{L}, B_{L}, C_{L}$ time-invariant matrices determining the dynamic and steady-state characteristics of the load. 


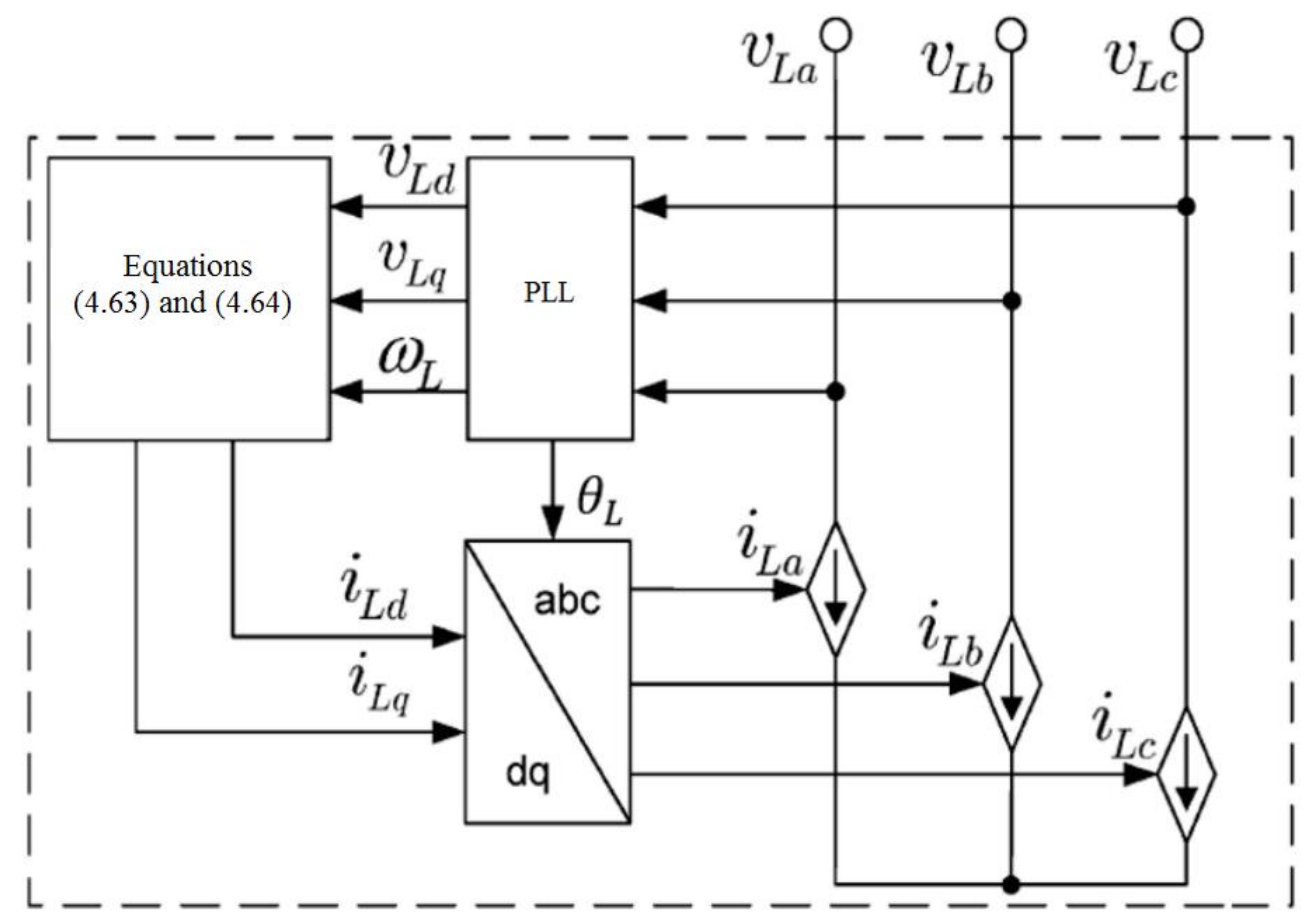

Fig. 4.19: Block diagram of the generic load model proposed in [138].

By solving (4.63) and (4.64), the load currents are obtained, as given by (4.65), where $X_{L}(0)$ denotes the initial state variables vector.

$$
\left[\begin{array}{l}
i_{L d}(t) \\
i_{L q}(t)
\end{array}\right]=C_{L}\left[e^{A_{L} t} X_{L}(0)+\int_{0}^{t} e^{A_{L}(t-\tau)} B_{L}\left[\begin{array}{l}
v_{L d}(t) \\
v_{L q}(t)
\end{array}\right] d \tau\right]
$$

From (4.63) and (4.64), the steady-state currents are:

$$
\left[\begin{array}{l}
i_{L d_{s S}} \\
i_{L q_{s s}}
\end{array}\right]=\left(-C_{L} A_{L}^{-1} B_{L}\right)\left[\begin{array}{l}
v_{L d_{S S}} \\
v_{L q_{s S}}
\end{array}\right]
$$

With a load-side voltage-oriented synchronous reference frame, $v_{L q_{s S}}=0$. The steady-state real and reactive powers of the load are given by:

$$
\left[\begin{array}{l}
P \\
Q
\end{array}\right]=\frac{3}{2} v_{L d_{s s}}\left[\begin{array}{c}
i_{L d_{S S}} \\
-i_{L q_{s s}}
\end{array}\right]
$$

Substituting (4.66) in (4.67), yields

$$
\left[\begin{array}{c}
P \\
-Q
\end{array}\right]=\frac{3}{2} v_{L d_{S S}}\left(-C_{L} A_{L}^{-1} B_{L}\right)\left[\begin{array}{c}
v_{L d_{S S}} \\
0
\end{array}\right]
$$


Assuming the product $\left(C_{L} A_{L}^{-1} B_{L}\right)$ to be a function of load-side frequency, $\omega_{L}, P$ and $Q$ can be expressed as:

$$
\begin{aligned}
& P=P_{o}\left(\frac{v_{L d_{S S}}}{V_{o}}\right)^{\alpha_{p}}\left(\frac{\omega_{L}}{\omega_{L o}}\right)^{\beta_{p}} \\
& Q=Q_{o}\left(\frac{v_{L d_{S S}}}{V_{o}}\right)^{\alpha_{q}}\left(\frac{\omega_{L}}{\omega_{L o}}\right)^{\beta_{q}}
\end{aligned}
$$

where $P_{0}$ and $Q_{0}$ stand for real and reactive power at nominal load voltage $\left(V_{0}\right)$ and nominal load frequency $\left(\omega_{L o}\right)$. The parameters $\beta_{p}$ and $\beta_{q}$ characterize the dependence of load powers on frequency, while the parameters $\alpha_{p}$ and $\alpha_{q}$ characterize the dependence of load powers on voltage magnitude depending on load type, as given in Table 4.2. In this model, constantimpedance load type is assumed, i.e., $\alpha_{p}=\alpha_{q}=2$. The matrices $A_{L}, B_{L}, C_{L}$ are given in Table 4.3. In the table, $d$ denotes damping of natural modes of the load model in $s^{-1}$ ( $d$ is inversely proportional to the settling time, $\left.t_{s}\right), \omega_{o}$ the oscillation frequency in $\mathrm{rad} / \mathrm{s}$, and $Y_{P}$ and $Y_{Q}$, given in (4.71) and (4.72), the real and reactive power indices of the load, respectively. The dynamic characteristics of the load is determined by $d$ and $\omega_{o}$ which, in turn, are equal to the real and imaginary parts of the eigenvalues of matrix $A_{L}$. On the other hand, $P_{o}, Q_{o}, \alpha_{p}, \alpha_{q}, \beta_{p}, \beta_{q}, V_{o}$ and $\omega_{L o}$ determine the steady-state characteristics of the load.

$$
\begin{aligned}
& Y_{P}=\frac{2}{3} \frac{1}{V_{o}^{2}}\left(\frac{\omega_{L}}{\omega_{L o}}\right)^{\beta p} P_{o} \\
& Y_{Q}=\frac{2}{3} \frac{1}{V_{o}^{2}}\left(\frac{\omega_{L}}{\omega_{L o}}\right)^{\beta q} Q_{o}
\end{aligned}
$$

Table 4.2: Dependence of load on voltage magnitude.

\begin{tabular}{lccc}
\hline Load type & $\boldsymbol{\alpha}_{\boldsymbol{p}}$ & $\boldsymbol{\alpha}_{\boldsymbol{q}}$ & Examples \\
\hline Constant Impedance & 2 & 2 & $\begin{array}{c}\text { Incandescent Lighting, electric stoves, and } \\
\text { water heaters }\end{array}$ \\
$\begin{array}{lccc}\text { Constant Current } \\
\text { Constant Power }\end{array}$ & 1 & 1 & $\begin{array}{c}\text { Controlled-current motor drives } \\
\text { Induction motor drives and controlled power } \\
\text { supplies }\end{array}$ \\
\hline
\end{tabular}




\subsubsection{Reduced-Order Load Model}

As shown in Table 4.3, the model proposed by [138] is a $4^{\text {th }}$-order state-space system with two uncontrollable modes (i.e., the controllability matrix is not full rank). By applying minimal realization technique, the matrices $A_{L}, B_{L}$ and $C_{L}$ are modified into $A_{L_{m}}, B_{L_{m}}$ and $C_{L_{m}}$ and the model order is reduced to 2 . The properties of the reduced-order model versus the $4^{\text {th }}$-order model are given in Table 4.3. The states $x_{1 m}$ and $x_{2 m}$ in the reduced-order model are fictitious states resulting from the minimal realization process. The reduced-order model has the same inputs and outputs as the $4^{\text {th }}$-order model. In the $4^{\text {th }}$-order model, $Y_{Q}$ assumes a negative value [138]. In the reduced-order model, in order to comply with the common sign convention for load reactive power, $Y_{Q}$ has been assumed to be positive and hence multiplied by -1 . Using both models, Fig. 4.20 gives the responses of load currents to connection of a balanced load at $\mathrm{t}=0.1 \mathrm{~s}$ for the load parameters given in Table 4.3. The figure shows that the characteristics of the reduced-order model are identical to those of the original $4^{\text {th }}$-order model.

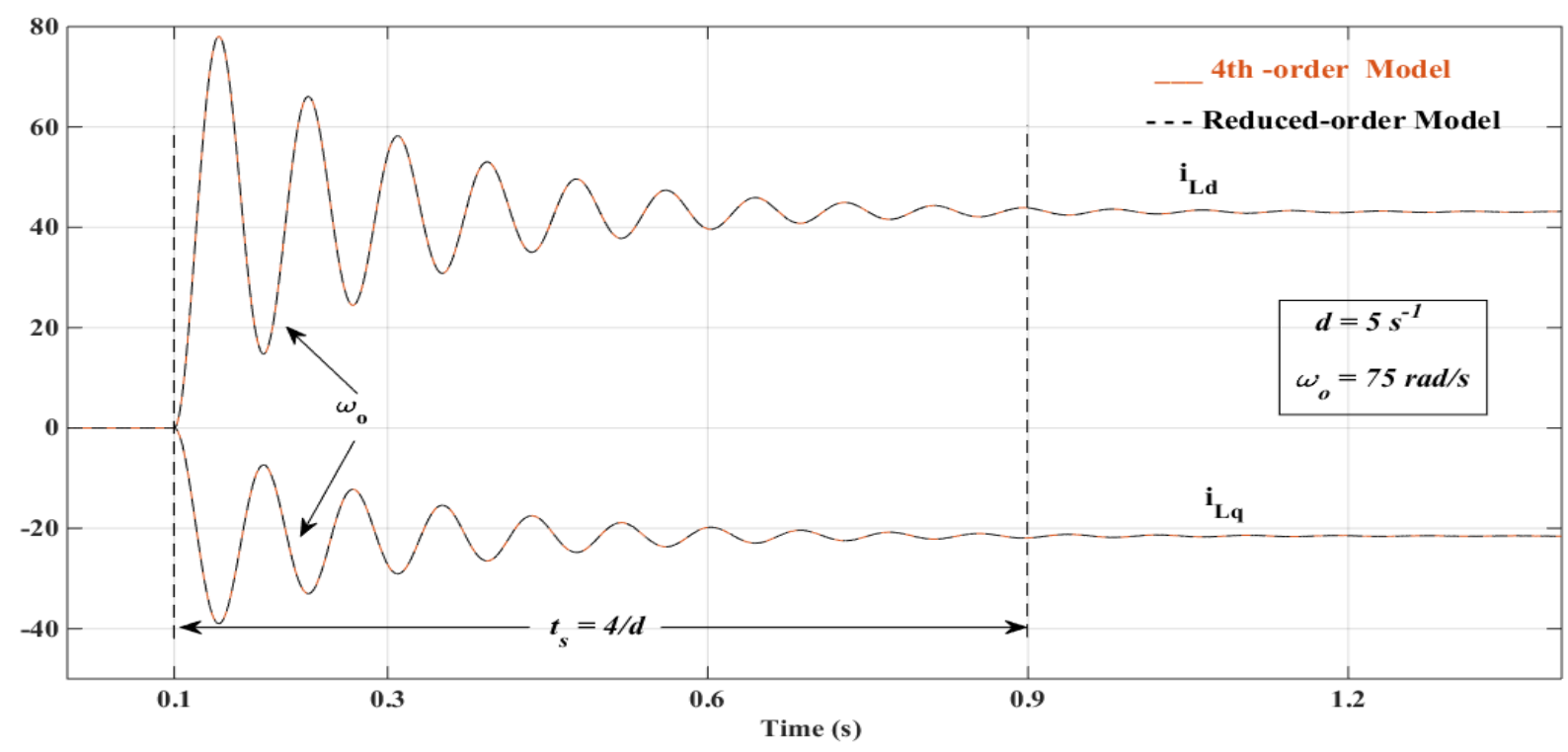

Fig. 4.20: Reduced-order model versus the original 4th -order model. 
Table 4.3: Characteristics of the generic load models.

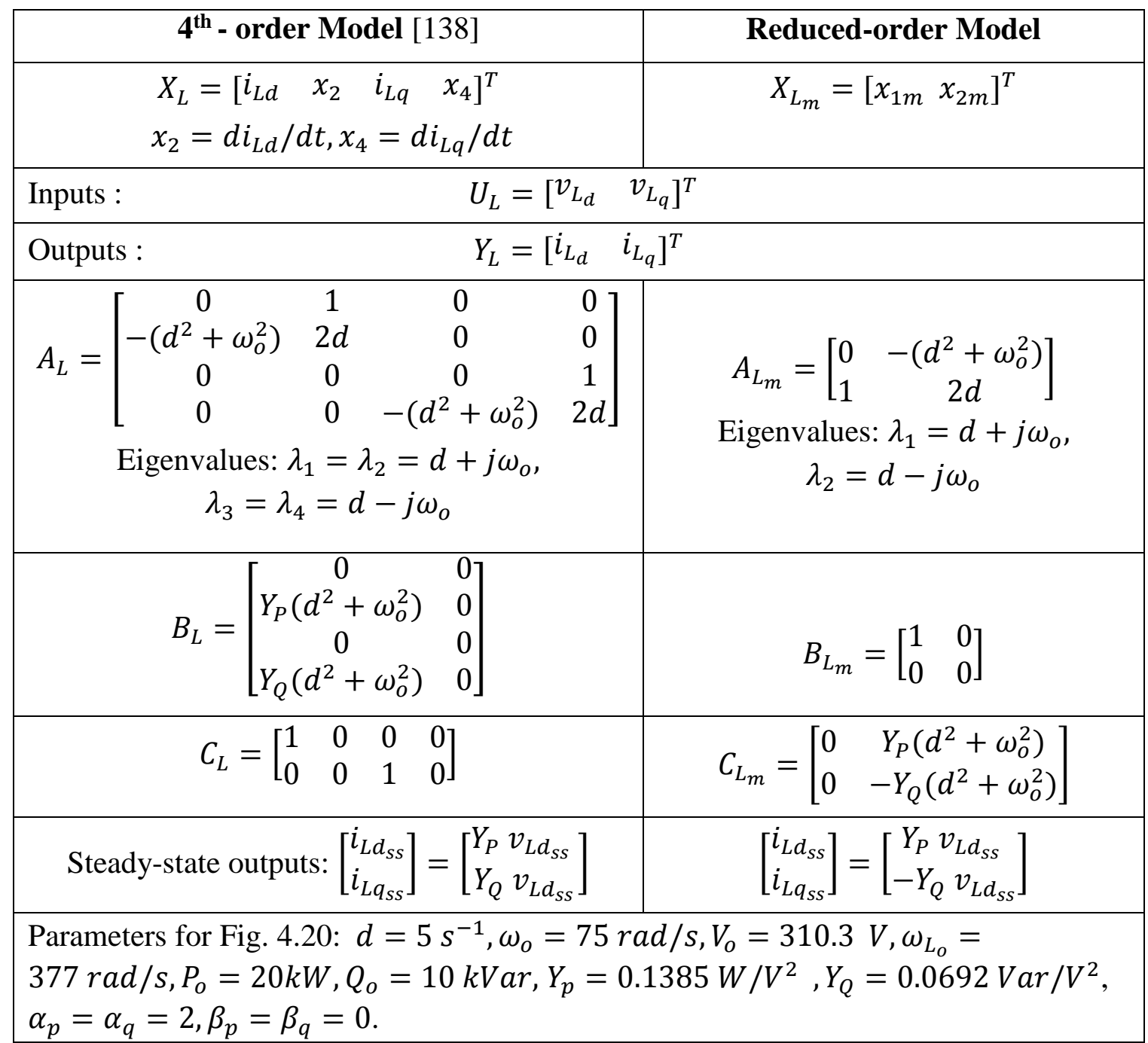

\subsubsection{Generic Load Model including Unbalanced Load Condition}

In Fig. 3.12, the proposed WECS supplies the load via a $\Delta / Y n$ transformer. As mentioned in subsection 4.8.1, under three-phase unbalanced load condition, the primary-side three-phase currents (i.e., $i_{a p}, i_{b p}$ and $i_{c p}$ ) contain only +ve and -ve seq components; hence, each component can be described by the reduced-order model of Table 4.3. In other words, the combination of unbalanced load and the $\Delta / Y n$ transformer, placed inside the dashed rectangle in Fig. 4.17, can be replaced by six dependent current sources ( 3 for each sequence), as shown in Fig. 4.21. 

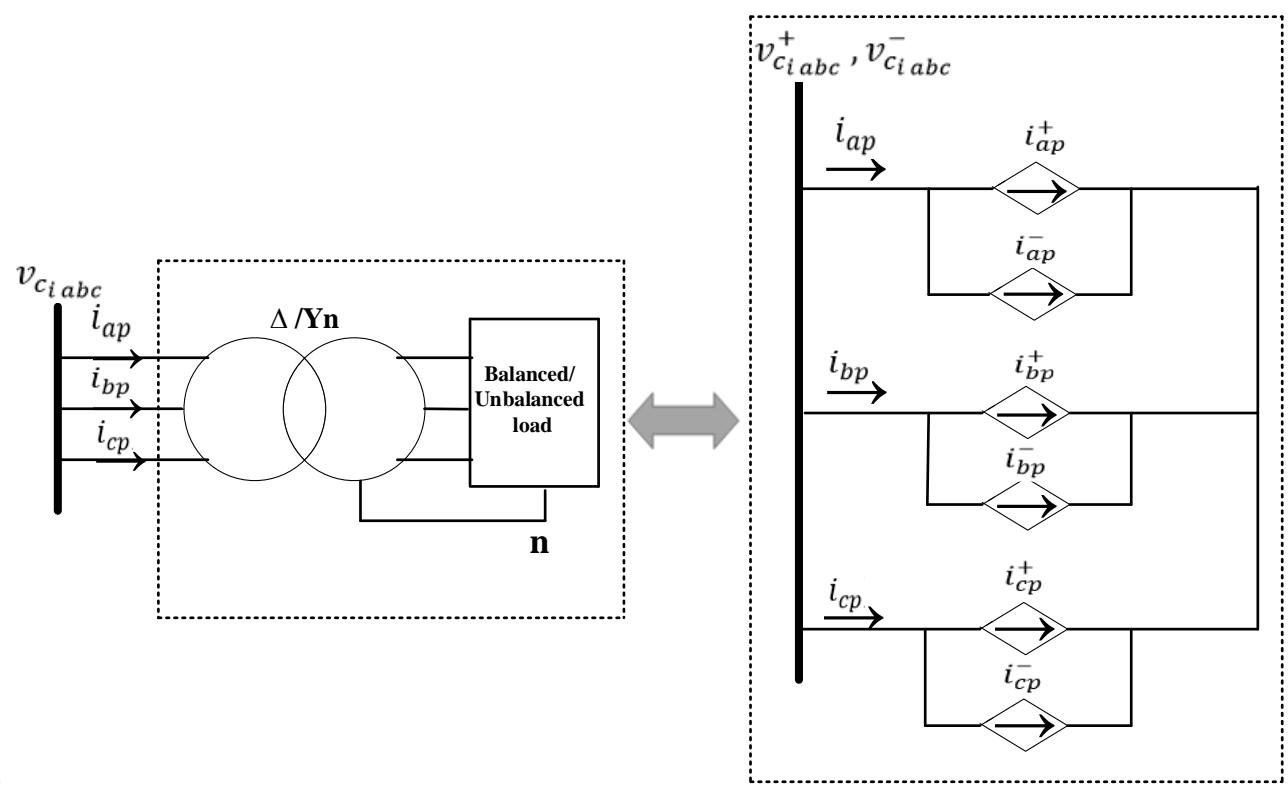

Fig. 4.21: Generic load model including unbalanced case.

In the presence of load imbalance, the function of the three-phase inverter is to maintain the symmetry of the three-phase output voltage applied to the load; hence, no -ve seq voltage is to be present across the capacitor in steady state, i.e., $v_{c i d_{s s}}^{-}=v_{c i q_{s s}}^{-}=0$. In other words, the CSI with capacitor filter represent a three-phase voltage source that supplies balanced voltages to an unbalanced three-phase load.

Based on (4.66)-(4.72), the steady-state active and reactive powers and power indices associated with +ve seq voltage and +ve seq current are given by (4.73) - (4.75).

$$
\begin{gathered}
{\left[\begin{array}{c}
P^{+} \\
Q^{+}
\end{array}\right]=\frac{3}{2} v_{c i d_{s S}}^{+}\left[\begin{array}{c}
i_{p d_{s S}}^{+} \\
-i_{p q_{s S}}^{+}
\end{array}\right]} \\
Y_{P}^{+}=\frac{2}{3} \frac{1}{V_{o}^{2}}\left(\frac{\omega_{L}}{\omega_{L o}}\right)^{\beta p} P_{o}^{+} \\
Y_{Q}^{+}=\frac{2}{3} \frac{1}{V_{o}^{2}}\left(\frac{\omega_{L}}{\omega_{L o}}\right)^{\beta q} Q_{O}^{+}
\end{gathered}
$$

Since $v_{c i d_{s s}}^{-}=0$, steady-state active and reactive powers produced by the multiplication of -ve seq voltage and -ve seq currents will be equal to zero. 
The interference between +ve seq voltage and -ve seq current produces a power component oscillating at twice the load-side frequency. This oscillating power is referred to as negative seq reactive power $\left(Q^{ \pm}\right)$and is given by (4.76) [140], where $V_{c_{i}}^{+}, \theta_{v_{c_{i}}}^{+}, I_{p}^{-}$and $\theta_{i_{p}}^{-}$present magnitudes and phase angles of +ve seq capacitor voltage and -ve seq load current, respectively.

$$
Q^{ \pm}=-3 V_{c_{i}}^{+} I_{p}^{-} \cos \left(2 \omega_{L} t+\theta_{v_{c_{i}}}^{+}-\theta_{i_{p}}^{-}\right)
$$

In this thesis, the peak value of $Q^{ \pm}$(i.e., $3 V_{c_{i}}^{+} I_{p}^{-}$), denoted by $Q^{-}$, will be adopted to obtain expressions for power indices, allowing to extend the reduced-order load model to unbalanced load case. In synchronous $d q$ reference frame, $Q^{-}$is expressed as:

$$
Q^{-}=\frac{3}{2} v_{c i d}^{+}\left(i_{p d}^{-}+j i_{p q}^{-}\right)=Q_{p d}^{-}+j Q_{p q}^{-}
$$

In (4.77), $Q_{p d}^{-}$and $Q_{p q}^{-}$represent the reactive powers associated with the -ve seq currents and are counterparts of $P^{+}$and $Q^{+}$, respectively, that are associated with the +ve seq currents.

In parallel with (4.73)-(4.75), the steady-state $d$-axis and $q$-axis powers and power indices associated with +ve seq voltage and -ve seq currents are given by (4.78)-(4.80).

$$
\begin{gathered}
{\left[\begin{array}{c}
Q_{p d}^{-} \\
Q_{p q}^{-}
\end{array}\right]=\frac{3}{2} v_{c i d_{s s}}^{+}\left[\begin{array}{c}
i_{p d_{s S}}^{-} \\
-i_{p q_{s S}}^{-}
\end{array}\right]} \\
Y_{Q_{p d}}^{-}=\frac{2}{3} \frac{1}{V_{o}^{2}}\left(\frac{\omega_{L}}{\omega_{L o}}\right)^{\beta p} Q_{p d o}^{-} \\
Y_{Q_{p q}}^{-}=\frac{2}{3} \frac{1}{V_{o}^{2}}\left(\frac{\omega_{L}}{\omega_{L o}}\right)^{\beta q} Q_{p q o}^{-}
\end{gathered}
$$

Based on the above equations, the reduced-order model introduced in Table 4.3 is extended, as shown in Table 4.4, to describe the dynamics of the load currents under unbalanced load conditions. In Table 4.4, $d$ and $\omega_{o}$ determine the dynamics of both +ve and -ve seq currents, while $P_{o}^{+}, Q_{o}^{+}, Q_{p d o}^{-}, Q_{p q o}^{-}, \beta_{p}, \beta_{q}, V_{o}$ and $\omega_{L o}$ correspond to the steady-state currents. Using the extended model, Fig. 4.22 gives the responses of load currents to connection of an unbalanced load to the load bus at $\mathrm{t}=0.1 \mathrm{~s}$ for the load parameters given in Table 4.4. 
It can be seen that the extended model is valid for both balanced and unbalanced load conditions. In case of balanced load, the differential equations associated with -ve seq currents are eliminated; hence, the system becomes identical to the reduced-order model shown in Table 4.3 (i.e., $i_{p d}^{-}=i_{p q}^{-}=0, i_{p d}^{+}=i_{L_{d}}, i_{p q}^{+}=i_{L_{q}}$ ).

The load unbalance factor (LUF) is defined as the ratio of magnitude of -ve seq current to that of +ve seq current, i.e.,

$$
L U F=\sqrt{\frac{\left(i_{p d}^{-}\right)^{2}+\left(i_{p q}^{-}\right)^{2}}{\left(i_{p d}^{+}\right)^{2}+\left(i_{p q}^{+}\right)^{2}}}=\sqrt{\frac{\left(Q_{p d o}^{-}\right)^{2}+\left(Q_{p q o}^{-}\right)^{2}}{\left(P_{o}^{+}\right)^{2}+\left(Q_{o}^{+}\right)^{2}}}
$$

Table 4.4: Characteristics of extended generic load model.

\begin{tabular}{|c|c|}
\hline State variables & $X_{L_{M}}=\left[\begin{array}{llll}x_{1 m}^{+} & x_{2 m}^{+} & x_{1 m}^{-} & x_{2 m}^{-}\end{array}\right]^{T}$ \\
\hline Inputs & $U_{L}=\left[\begin{array}{ll}v_{c i d}^{+} & v_{c i q}^{+}\end{array}\right]^{T}$ \\
\hline Outputs & $Y_{L_{M}}=\left[\begin{array}{llll}i_{p d}^{+} & i_{p q}^{+} & i_{p d}^{-} & i_{p q}^{-}\end{array}\right]^{T}$ \\
\hline \multirow{3}{*}{ Model Matrices } & $A_{L_{M}}=\left[\begin{array}{cccc}0 & -\left(d^{2}+\omega_{o}^{2}\right) & 0 & 0 \\
1 & 2 d & 0 & 0 \\
0 & 0 & 0 & -\left(d^{2}+\omega_{o}^{2}\right) \\
0 & 0 & 1 & 2 d\end{array}\right]$ \\
\hline & $B_{L_{M}}=\left[\begin{array}{lllllll}1 & 0,0 & 0,1 & 0,0 & 0\end{array}\right]^{T}$ \\
\hline & $C_{L_{M}}=\left[\begin{array}{cccc}0 & Y_{P}^{+}\left(d^{2}+\omega_{o}^{2}\right) & 0 & 0 \\
0 & -Y_{Q}^{+}\left(d^{2}+\omega_{o}^{2}\right) & 0 & 0 \\
0 & 0 & 0 & Y_{Q_{p d}}^{-}\left(d^{2}+\omega_{o}^{2}\right) \\
0 & 0 & 0 & -Y_{Q_{p q}}^{-}\left(d^{2}+\omega_{o}^{2}\right)\end{array}\right]$ \\
\hline Steady- state currents & $\begin{array}{c}i_{p d_{s s}}^{+}=Y_{P}^{+} v_{c i d}^{+}, i_{p q_{s s}}^{+}=-Y_{Q}^{+} v_{c i d}^{+} \\
i_{p d_{s s}}^{-}=Y_{Q_{p d}}^{-} v_{c i d}^{+}, i_{p q_{s s}}^{-}=-Y_{Q_{p q}}^{-} v_{c i d}^{+}\end{array}$ \\
\hline Parameter for Fig. 4.22 & $\begin{array}{c}d=10 \mathrm{~s}^{-1}, \omega_{o}=377 \mathrm{rad} / \mathrm{s}, V_{o}=310.3 \mathrm{~V}, P_{o}^{+}=20 \mathrm{~kW}, \\
Y_{P}^{+}=0.1385 \mathrm{~W} / \mathrm{V}^{2}, Q_{o}^{+}=10 \mathrm{kVar}, Y_{Q}^{+}=0.0692 \mathrm{Var} / \mathrm{V}^{2}, \\
Q_{p d o}^{-}=-5.08 \mathrm{kVar}, Y_{Q_{p d}}^{-}=-0.0352 \mathrm{Var} / \mathrm{V}^{2}, \\
Q_{p q o}^{-}=-6.04 \mathrm{kVar}, Y_{Q_{p q}}^{-}=-0.0418 \mathrm{Var} / \mathrm{V}^{2} \\
\alpha_{p}=\alpha_{q}=2, \beta_{p}=\beta_{q}=0 .\end{array}$ \\
\hline
\end{tabular}



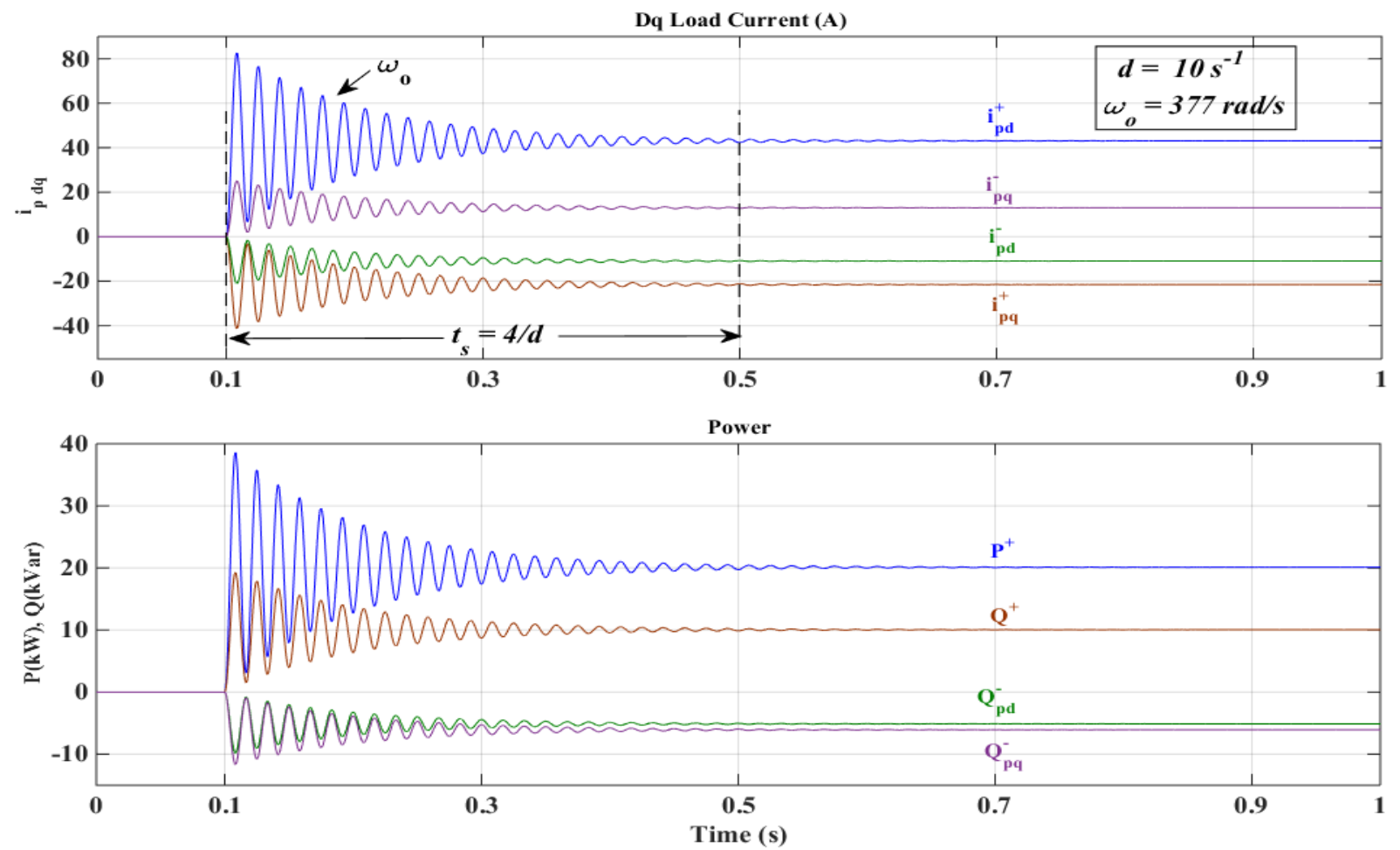

Fig. 4.22: Load currents and powers using the extended generic load model.

\subsection{DC-Link Model}

In the proposed system, the generator side combined with storage side is linked with the load side through the dc-link inductor, as shown in Fig. 4.23.

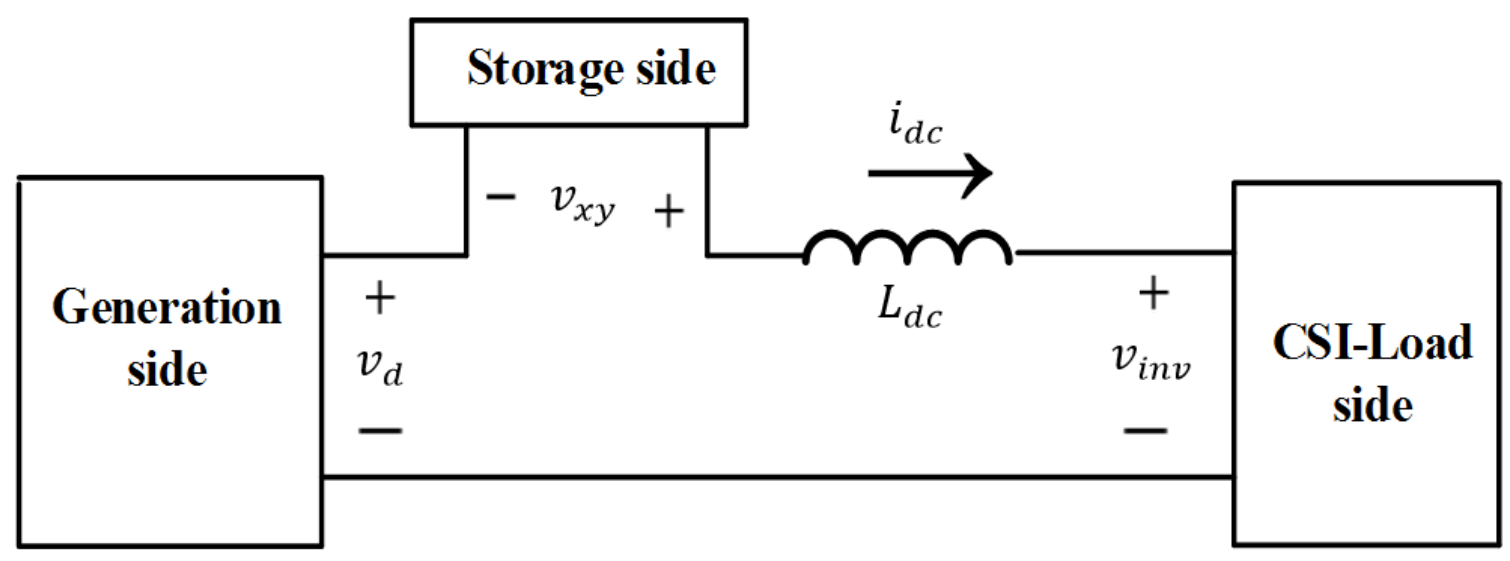

Fig. 4.23: Configuration of the dc link. 
Based on Fig. 4.23, the dynamics of dc-link current is given by:

$$
\frac{d}{d t} i_{d c}=\frac{1}{L_{d c}}\left(v_{d}+v_{x y}-v_{i n v}\right)
$$

where $v_{d}, v_{x y}$, and $v_{i n v}$ were defined in (4.36), (4.44), and (4.61), respectively.

\subsection{Dynamic Model of the Proposed Wind Energy Conversion System}

In the previous sections, the dynamic equations of different components of the proposed system were derived. Using these equations, the dynamic and steady-state models of the proposed WECS are developed in this section.

\subsubsection{State Space Equations}

In general, a linear-time invariant system is described by the following set of state-space equations:

$$
\begin{aligned}
& \dot{X}=A X+B U \\
& Y=C X+D U
\end{aligned}
$$

where $X, U$, and $Y$ are the state variables vector, inputs vector, and output vector of the system, respectively. $A, B, C$, and $D$ are the matrices for multi-input, multi-output (MIMO) system. By combining all the equations derived in pervious sections, the entire system of the proposed CSI-based WECS is described by a $20^{\text {th }}$-order state-space system given in (4.84). The derivation process of the overall system model is presented in Appendix D. The dynamics of the system is described by 20 first-order differential equations: 3 for the wind turbine shaft, 6 for the self-excited induction generator, 2 for the battery-side low-pass filter, 4 for the CSIside capacitor filter, 4 for the extended generic load model, and 1 for the dc link. In case of balanced load condition, the four differential equations associated with the -ve seq components of CSI-side filter capacitor voltages and transformer primary-side currents are eliminated; hence, the order of the overall system is reduced to 16 . 


$$
\begin{aligned}
& \left(\frac{d}{d t} \omega_{m}=\frac{1}{J_{m}}\left[\rho \pi r^{2}\left(\left(\frac{30 v_{w}^{3}}{\omega_{m}^{2} r}-\frac{2.35 v_{w}^{2}}{\omega_{m}}\right) e^{\left(-\frac{21 v_{w}}{\omega_{m} r}+0.74\right)}+0.0034 r v_{w}\right) v_{w}-K_{s e} \delta \theta-D_{\text {se }}\left(\omega_{m}-\frac{\omega_{r}}{P n_{\text {gear }}}\right)\right]\right. \\
& \frac{d}{d t} \omega_{r}=\frac{P}{J_{g}}\left[\frac{1}{n_{\text {gear }}} K_{s e} \delta \theta+\frac{1}{n_{\text {gear }}} D_{\text {se }}\left(\omega_{m}-\frac{\omega_{r}}{P n_{\text {gear }}}\right)-\frac{3 P L_{m}}{2}\left(i_{q s} i_{d r}^{\prime}-i_{d s} i_{q r}^{\prime}\right)\right] \\
& \frac{d}{d t}(\delta \theta)=\omega_{m}-\frac{\omega_{r}}{P n_{\text {gear }}} \\
& \frac{d}{d t} i_{q s}=\frac{1}{k_{s}}\left[R_{s} L_{r}^{\prime} i_{q s}+\left(\omega_{e}\left(L_{s} L_{r}^{\prime}-L_{m}^{2}\right)+\omega_{r} L_{m}^{2}\right) i_{d s}-R_{r}^{\prime} L_{m} i_{q r}^{\prime}+\omega_{r} L_{m} L_{r}^{\prime} i_{d r}^{\prime}-L_{r}^{\prime} v_{c_{g q}}\right] \\
& \frac{d}{d t} i_{d s}=\frac{1}{k_{s}}\left[\left(\omega_{e}\left(L_{m}^{2}-L_{s} L_{r}^{\prime}\right)-\omega_{r} L_{m}^{2}\right) i_{q s}+R_{s} L_{r}^{\prime} i_{d s}-\omega_{r} L_{m} L_{r}^{\prime} i_{q r}^{\prime}-R_{r}^{\prime} L_{m} i_{d r}^{\prime}-L_{r}^{\prime} v_{c g d}\right] \\
& \frac{d}{d t} i_{q r}^{\prime}=\frac{1}{k_{s}}\left[-R_{s} L_{m} i_{q s}-\omega_{r} L_{m} L_{s} i_{d s}+R_{r}^{\prime} L_{s} i_{q r}^{\prime}+\left(\omega_{e}\left(L_{s} L_{r}^{\prime}-L_{m}^{2}\right)-L_{s} L_{r}^{\prime} \omega_{r}\right) i_{d r}^{\prime}+L_{m} v_{c_{g q}}\right] \\
& \frac{d}{d t} i_{d r}^{\prime}=\frac{1}{k_{s}}\left[\omega_{r} L_{m} L_{s} i_{q s}-R_{s} L_{m} i_{d s}+\left(\omega_{e}\left(L_{m}^{2}-L_{s} L_{r}^{\prime}\right)+L_{s} L_{r}^{\prime} \omega_{r}\right) i_{q r}^{\prime}+R_{r}^{\prime} L_{s} i_{d r}^{\prime}+L_{m} v_{c_{g d}}\right] \\
& \frac{d}{d t} v_{c_{g q}}=-\frac{1}{C_{g}} i_{q s}-\omega_{e} v_{c_{g d}} \\
& \frac{d}{d t} v_{c_{g d}}=-\frac{1}{C_{g}}\left(i_{d s}+\frac{2 \sqrt{3}}{\pi} d_{b} i_{d c}\right)+\omega_{e} v_{c_{g q}} \\
& \frac{d}{d t} i_{b a t}=\frac{1}{L_{b}}\left(E_{o}-K_{E}(1-S o C)-R_{o}\left(1+A_{0}(1-S o C)\right) i_{b a t}-v_{c_{b}}\right) \\
& \frac{d}{d t} v_{c_{b}}=\frac{1}{c_{b}}\left(i_{b a t}-\left(2 d_{A}-1\right) i_{d c}\right) \\
& \frac{d}{d t} v_{c i d}^{+}=\frac{1}{C_{i}}\left(G m_{i d}^{+} i_{d c}-Y_{P}^{+}\left(d^{2}+\omega_{o}^{2}\right) x_{2 m}^{+}\right)+\omega_{L} v_{c i q}^{+} \\
& \frac{d}{d t} v_{c i q}^{+}=\frac{1}{C_{i}}\left(G m_{i q}^{+} i_{d c}+Y_{Q}^{+}\left(d^{2}+\omega_{o}^{2}\right) x_{2 m}^{+}\right)-\omega_{L} v_{c i d}^{+} \\
& \frac{d}{d t} v_{c i d}^{-}=\frac{1}{C_{i}}\left(G m_{i d}^{-} i_{d c}-Y_{Q_{p d}}^{-}\left(d^{2}+\omega_{o}^{2}\right) x_{2 m}^{-}\right)-\omega_{L} v_{c i q}^{-} \\
& \frac{d}{d t} v_{c i q}^{-}=\frac{1}{C_{i}}\left(G m_{i q}^{-} i_{d c}+Y_{Q_{p q}}^{-}\left(d^{2}+\omega_{o}^{2}\right) x_{2 m}^{-}\right)+\omega_{L} v_{c i d}^{-} \\
& \frac{d}{d t} x_{1 m}^{+}=-\left(d^{2}+\omega_{o}^{2}\right) x_{2 m}^{+}+v_{c i d}^{+} \\
& \frac{d}{d t} x_{2 m}^{+}=x_{1 m}^{+}+2 d x_{2 m}^{+} \\
& \frac{d}{d t} x_{1 m}^{-}=-\left(d^{2}+\omega_{o}^{2}\right) x_{2 m}^{-}+v_{c i d}^{+} \\
& \frac{d}{d t} x_{2 m}^{-}=x_{1 m}^{-}+2 d x_{2 m}^{-} \\
& \frac{d}{d t} i_{d c}=\frac{1}{L_{d c}} \frac{3 \sqrt{3}}{\pi} d_{b} v_{c g d}+\frac{1}{L_{d c}}\left(2 d_{A}-1\right) v_{c_{b}}-\frac{1.5}{L_{d c}} G\left(m_{i d}^{+} v_{c i d}^{+}+m_{i q}^{+} v_{c i q}^{+}+m_{i d}^{-} v_{c i d}^{-}+m_{i q}^{-} v_{c i q}^{-}\right)
\end{aligned}
$$


The schematic diagram of the proposed system's overall dynamic model is illustrated in Fig. 4.24. The model is characterized by 7 inputs $U, 20$ state variables $X$, and 6 outputs $Y$. The vectors $X$ and $U$ are:

$$
\begin{gathered}
X=\left[\begin{array}{ccccccccccc}
\omega_{m} & \omega_{r} & \delta \theta & i_{q s} & i_{d s} & i_{q r}^{\prime} & i_{d r}^{\prime} & v_{c_{g q}} & v_{c_{g d}} & i_{b a t} & v_{c_{b}} \\
v_{c i d}^{+} & v_{\text {ciq }}^{+} & v_{\text {cid }}^{-} & v_{c i q}^{-} & x_{1 m}^{+} & x_{2 m}^{+} & x_{1 m}^{-} & x_{2 m}^{-} & i_{d c}
\end{array}\right]^{T} \\
U=\left[\begin{array}{llllllll}
v_{w} & d_{b} & d_{A} & m_{i d}^{+} & m_{i q}^{+} & m_{i d}^{-} & m_{i q}^{-}
\end{array}\right]^{T}
\end{gathered}
$$

The state variables to be controlled are selected as the output of the system, i.e.,

$$
Y=\left[\begin{array}{llllll}
\omega_{r} & v_{\text {cid }}^{+} & v_{\text {ciq }}^{+} & v_{\text {cid }}^{-} & v_{\text {ciq }}^{-} i_{d c}
\end{array}\right]^{T}
$$

The system matrices $A$ and $B$ are provided in Appendix $E$.

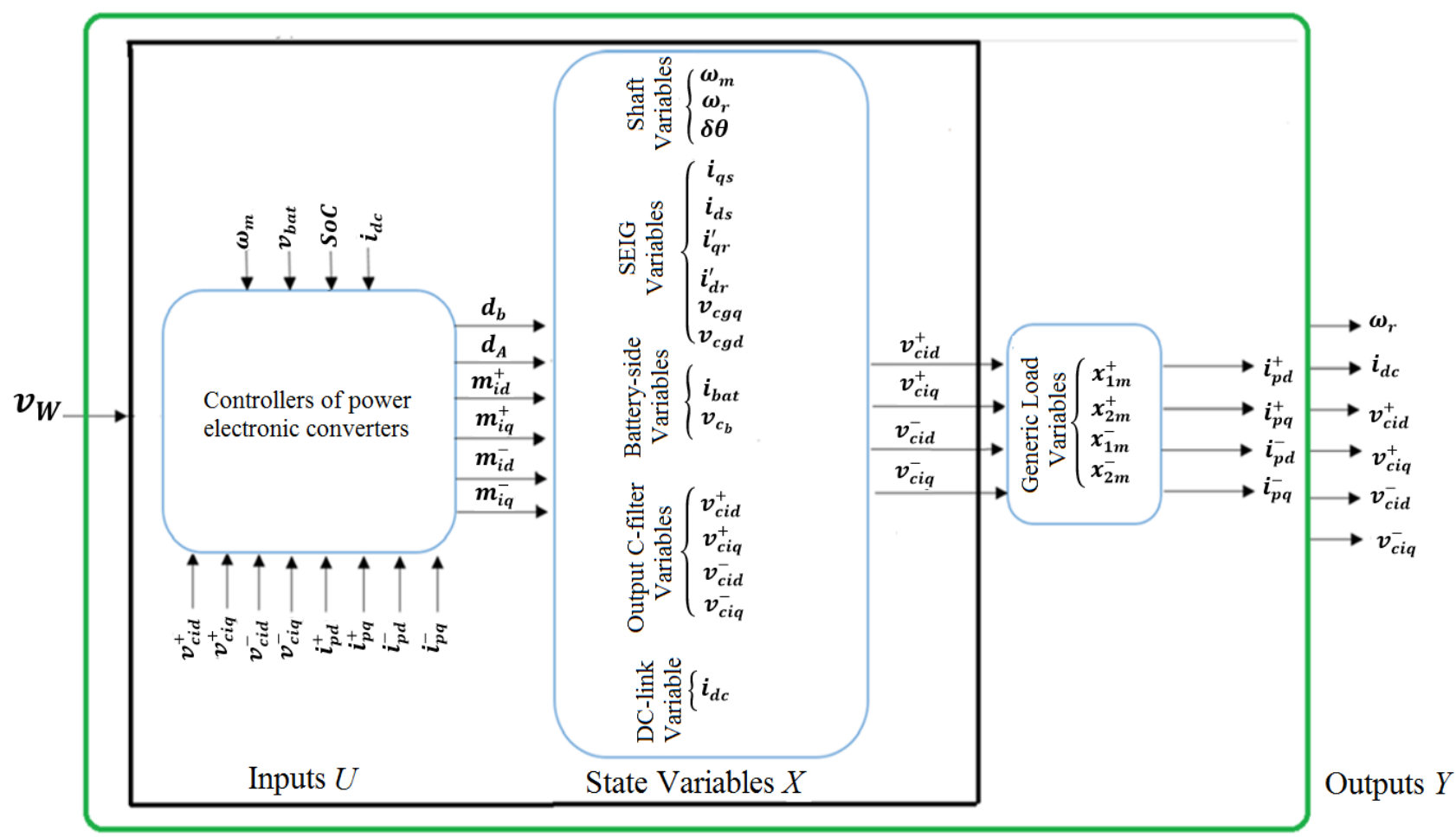

Fig. 4.24: Inputs, state variables, and outputs of the proposed WECS.

\subsubsection{Steady-State Equations}

The steady-state equations of the system are obtained by setting the right-hand sides of the equations given in (4.84) to zero, resulting in (4.88). 


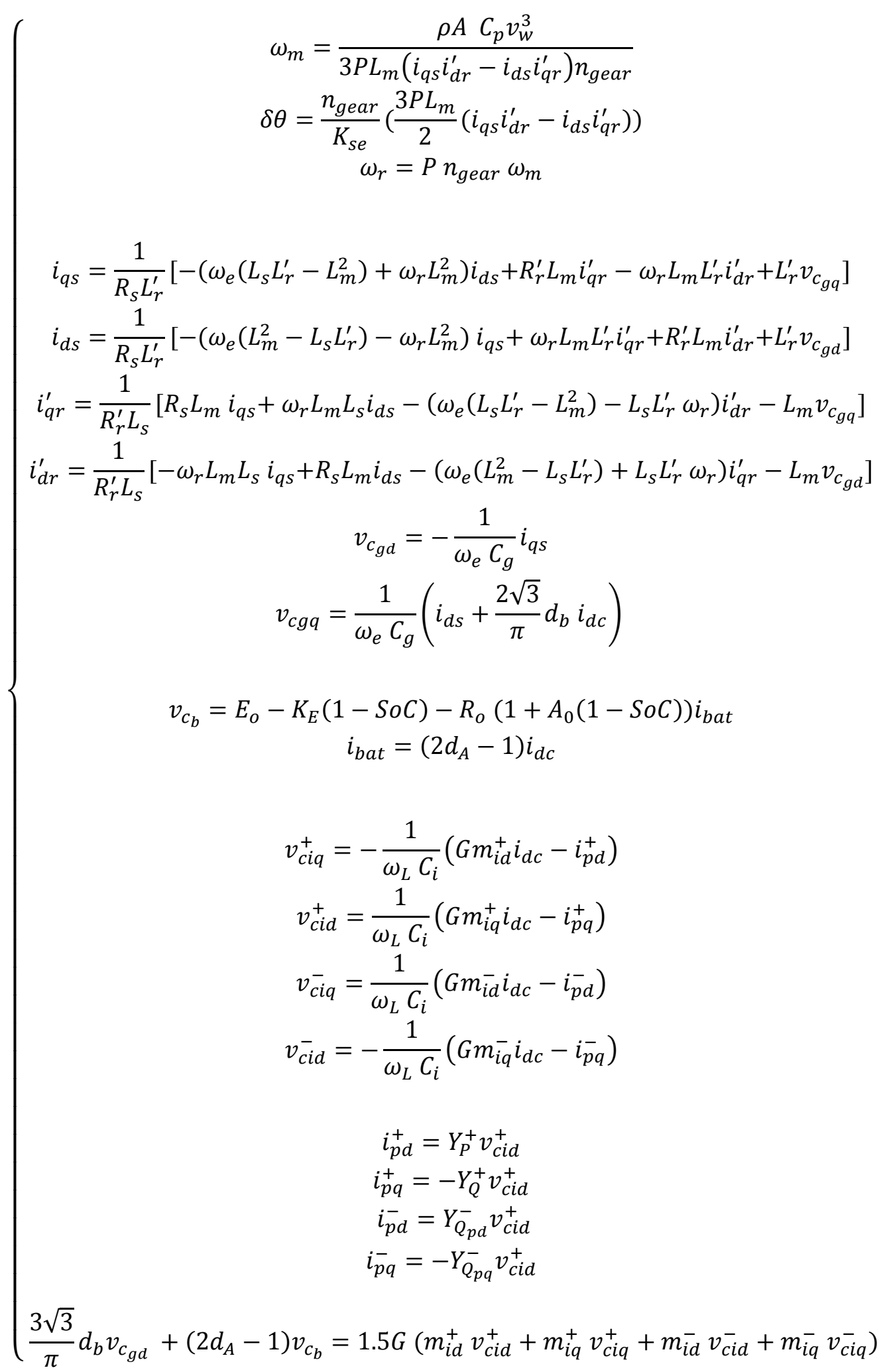


The twenty equations in (4.88) can be used to calculate the steady-state values of the system variables according to an arbitrary set of system inputs. Among the system inputs stated in (4.86), wind speed $\left(v_{w}\right)$ is the only uncontrolled input. The remaining inputs depend on the specific control algorithms employed. Among the state variables stated in (4.85), $v_{\text {ciq }}^{+}$is equal to zero in load-side voltage-oriented synchronous frame. Similarly, $v_{c g q}$ is equal to zero in generator-voltage-oriented synchronous frame. As mentioned in subsection 4.9.3, $v_{c i d}^{-}$and $v_{\text {ciq }}^{-}$are both equal to zero at steady-state operation, since the objective of load-side controllers is to compensate for voltage imbalance at load-side bus and hence no -ve seq voltage components exist across the output C-filter.

The steady-state values are obtained under the following assumptions:

- The wind turbine is operated under maximum power point tracking scheme.

- The mechanical losses are assumed to be $1 \%$ of the turbine's rated power [141]; hence, they are neglected.

- Due to the very high efficiency of power electronic devices, their conduction and switching losses are neglected.

- The generator-side cable losses are neglected.

- The transformer losses are considered as part of the load demand.

\subsection{Verification of the Overall Model}

The purpose of this section is to study the behavior of the system's output variables in response to variations of the input variables. Due to system complexity, it is decomposed into three subsystems: Wind-Turbine Generation (WTG) subsystem, Energy Storage (ES) Subsystem, and Current-Sourced Inverter-Load (CSI-Load) subsystem. The verification is conducted for each subsystem separately. State-space matrices of each subsystem as well as the entire system are given in Appendix E. The structure of the three subsystems are described in the following subsections. 


\subsubsection{Wind Turbine Generation (WTG) Subsystem}

The structure of the WTG subsystem is shown in Fig. 4.25. The input variables are the uncontrolled wind speed $\left(v_{w}\right)$, the adjustable control variable of the buck converter $\left(d_{b}\right)$, and the dc-link current $\left(i_{d c}\right)$, which is regulated by the ES subsystem. The dc current required from the generator (i.e., $i_{d c r}=d_{b} i_{d c}$ ) represent the load of the WTG subsystem. The dc voltage produced by the buck converter $\left(v_{d}=d_{b} v_{d c}\right)$ can be taken as the output of the subsystem. Thus, with respect to the combination of ES and CSI-Load subsystems, the WTG subsystem can be looked at as a variable dc power supply. The active power produced by the generator (i.e., $P_{g}$ ) can also be considered as output of the subsystem.

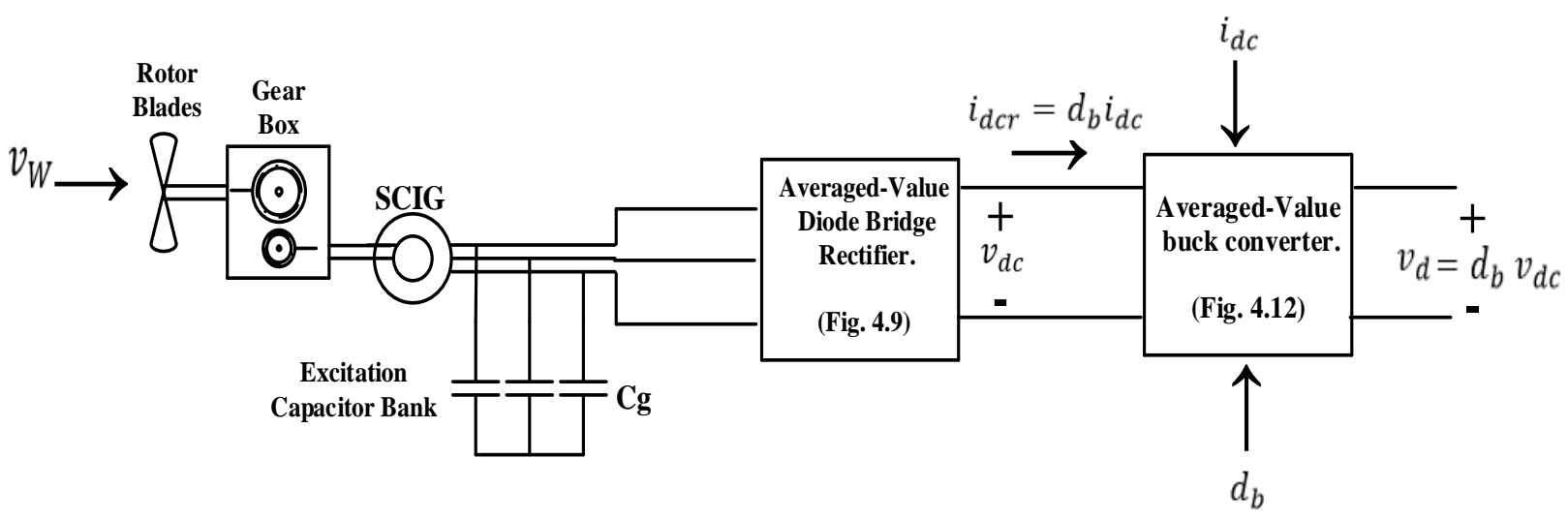

Fig. 4.25: WTG Subsystem.

\subsubsection{Energy Storage (ES) Subsystem}

As illustrated in Fig. 4.26, the input variables to ES subsystem are the variable dc voltage applied by WTG subsystem $\left(v_{d}\right)$, the adjustable control variable of the reduced H-bridge converter $\left(d_{A}\right)$, and the average dc-side voltage of the current-sourced inverter $\left(v_{\text {inv }}\right)$, which is reflected from the load side. Since the function of ES subsystem is to regulate the dc-link current, this current represents the output of the subsystem. Assuming a lossless system, $v_{d}$ and $v_{i n v}$ can be approximated by $P_{m} / i_{d c}$ and $P_{L} / i_{d c}$, respectively, where $P_{m}$ and $P_{L}$ are the wind turbine mechanical power and the load power, respectively. 


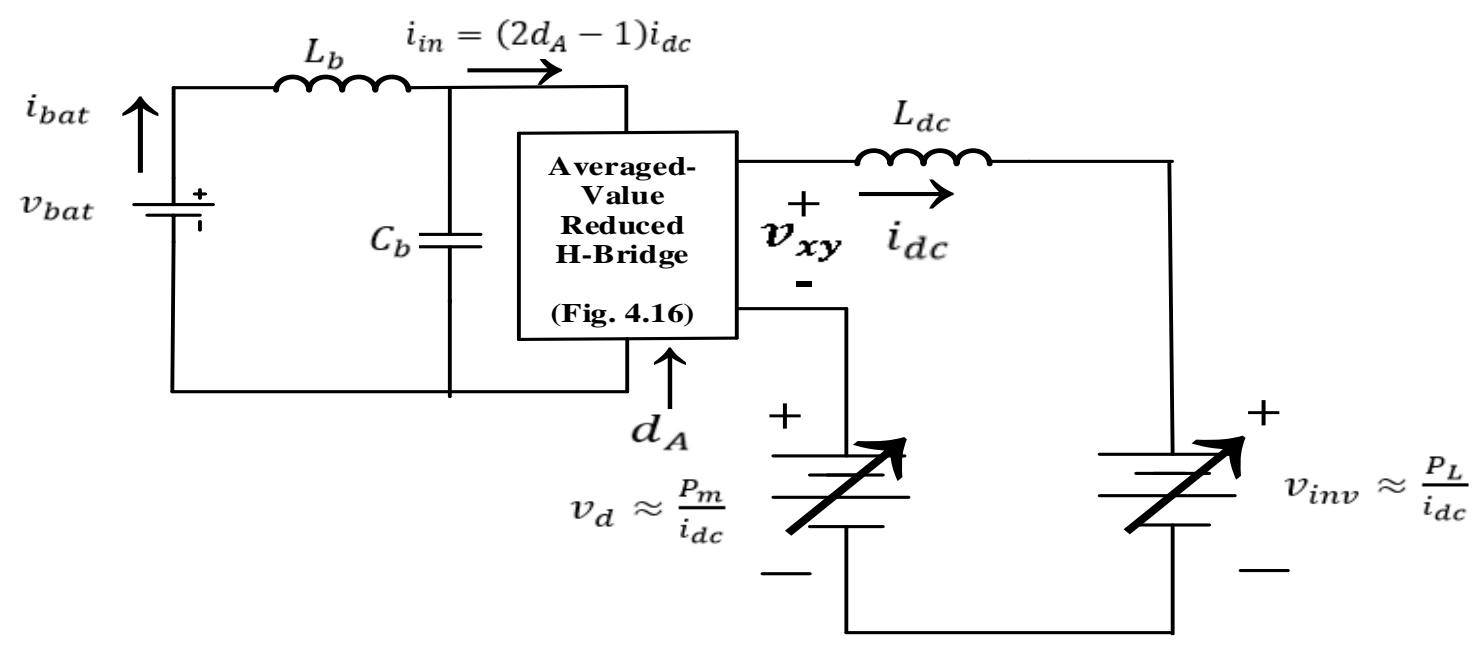

Fig. 4.26: ES Subsystem.

\subsubsection{Current-Sourced Inverter-Load (CSI-Load) Subsystem}

Fig. 4.27 illustrates the structure of the CSI-Load subsystem. The combination of the WTG and ES subsystems are assumed to be a dispatchable unit, and hence can be represented by a conditioned energy source, supplying a regulated dc current to the CSI-Load subsystem. The modulation indices of the current source inverter (i.e., $m_{i d}^{+}, m_{i q}^{+}, m_{i d}^{-}, m_{i q}^{-}$) are the adjustable input variables to the CSI-Load subsystem. The function of the current source inverter is to control the voltage magnitude and frequency at the load bus under balanced and unbalanced load. Therefore, the $d q$ capacitor voltages (i.e., $v_{c i d}^{+}, v_{c i q}^{+}, v_{c i d}^{-}, v_{c i q}^{-}$) represent the output of the CSI-Load subsystem.

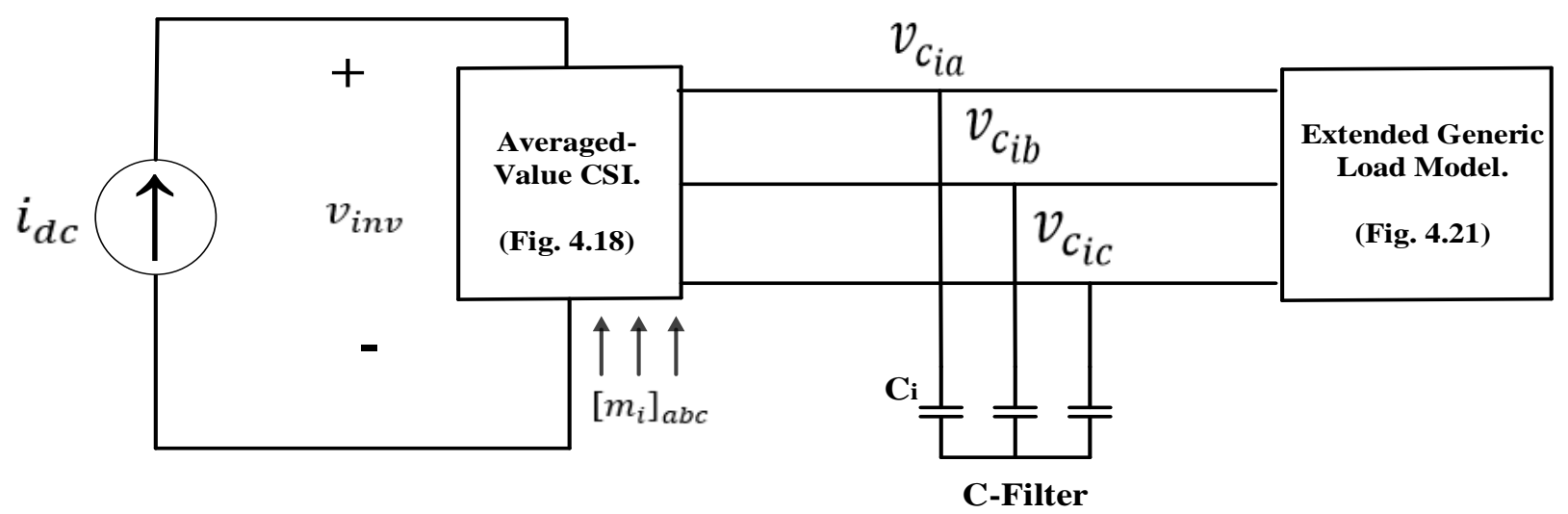

Fig. 4.27: CSI-Load Subsystem. 
In the following subsections, the starting operation of the system will be simulated first. Then, inputs and outputs of average and switching models of power electronic converters will be compared. Finally, the dynamic characteristics of the system will be investigated by examining its step responses. Simulation results are obtained based on switching and average models of a 20kW wind turbine system. Both models are built in Matlab/Simulink environment based on the system's parameters provided in Appendix A (Table A.2). In the following simulations, the wind speed is considered as a constant signal. The realistic wind speed model described in section 4.1 will be implemented in chapter 5 .

\subsubsection{Starting Operation of the Wind Turbine System}

The process of self-excitation in induction machines has been studied for over eighty years (since 1935) [142] and well-illustrated in the literatures through simulation and experiments [29],[124],[143]. When an induction machine, excited by an appropriate capacitor across its stator terminals, is driven by an external prime mover, a voltage will appear at its terminals. The starting operation of the WTG subsystem is investigated with the input parameters given in Table 4.5 .

Table 4.5: Input parameters for WTG subsystem used for starting simulation.

\begin{tabular}{|c|c|c|}
\hline Wind speed $(\mathbf{m} / \mathbf{s})$ & Buck duty cycle & DC-link current $(\mathbf{A})$ \\
\hline 12 & $d_{b}=0.63$ & $i_{d c}=51$ \\
\hline
\end{tabular}

In Fig. 4.28, two starting scenarios of the generator are simulated. In the first scenario, simulation starts with zero shaft speed, with no load connected to the generator (i.e., $d_{b}=0$ ). Once the shaft speed reaches the rated speed of $1 \mathrm{pu}$, rated load is connected to the generator by switching the buck duty cycle to 0.63 . The self-excitation process starts at $t=1.95 \mathrm{~s}$, after which the generated voltage builds up until it reaches steady-state value, where full excitation is achieved. This scenario is referred to as unloaded starting. In the second scenario, simulation starts with zero shaft speed, with rated load connected to the generator (i.e., $d_{b}=0.63$ ). Compared to the unloaded starting, the loaded starting requires a longer time for self-excitation process to start and thereby for full excitation to take place. This scenario is referred to as loaded starting. From the simulation responses, one can notice that the loaded starting scenario 
experiences very high power transients (with a power overshoot of around 90\%) compared with the unloaded starting (with a power overshoot of around $30 \%$ ).
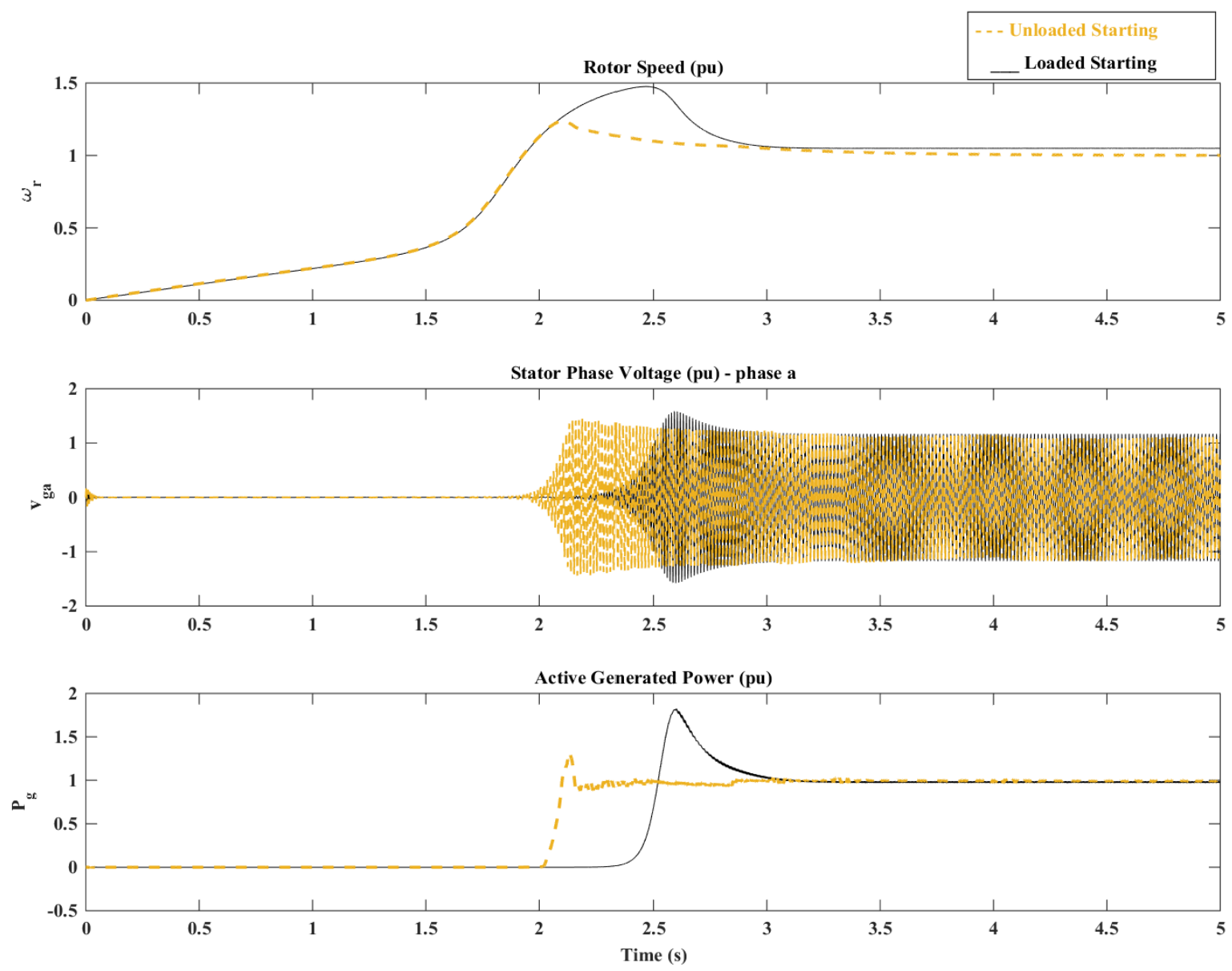

Fig. 4.28: Starting of the wind turbine under unloaded/loaded conditions.

\subsubsection{Average model versus Switching model for Power Electronics Converters}

In this subsection, the inputs and outputs of average models of power electronic converters, namely, diode-bridge rectifier, buck converter, reduced $\mathrm{H}$-bridge converter and CSI, are compared with those of switching models. The system is running at the steady-state operating point described in Table 4.6.

Fig. 4.29 illustrates the ac-side current (phase a) and dc-side voltage of diode bridge rectifier. One can notice a very small phase shift between the average model current and fundamental component of switching model current. This is caused by the effect of the dc-side capacitor $\left(C_{d c}\right)$ which was neglected in developing the average model. In other words, the average model 
line current is in phase with the supply phase voltage, while the fundamental component of switching model current is leading the supply phase voltage.

The waveforms, illustrated in Fig. 4.30, Fig. 4.31, and Fig. 4.32, show that the average models of power electronic converters represent to a very good approximation the corresponding switching models, with the distinct difference that high-frequency switching harmonics are not present in the average models.

Table 4.6: Input, output and parameter values at the operating point for steady-state analysis.

\begin{tabular}{|c|c|c|c|}
\hline$v_{w}=12 \mathrm{~m} / \mathrm{s}$ & $d_{b}=0.63$ & $d_{A}=0.108$ & $m_{i d}^{+}=0.1945$ \\
\hline$m_{i q}^{+}=0.233$ & $m_{\text {id }}^{-}=-0.049$ & $m_{i q}^{-}=0.059$ & $\omega_{r}=379.5 \mathrm{rad} / \mathrm{s}$ \\
\hline$P_{g}=20 \mathrm{~kW}$ & $Q_{g}=-11.5 \mathrm{kVar}$ & $i_{d c}=51 \mathrm{~A}$ & $P_{b a t}=-16 \mathrm{~kW}$ \\
\hline$P_{L}^{+}=4 \mathrm{~kW}$ & $Q_{L}^{+}=2 \mathrm{kVar}$ & $Q_{p d}^{-}=1.02 \mathrm{~kW}$ & $Q_{p q}^{-}=1.21 \mathrm{kVar}$ \\
\hline$d=100 \mathrm{~s}^{-1}$ & $\omega_{o}=75 \mathrm{rad} / \mathrm{s}$ & $v_{c i d}^{+}=310.3 \mathrm{~V}$ & $v_{c i q}^{+}=0$ \\
\hline$v_{\text {cid }}^{-}=v_{\text {ciq }}^{-}=0$ & $v_{\text {bat }}=393.3 \mathrm{~V}$ & SoC $=50 \%$ & $f_{s}=5.1 \mathrm{kHz}$ \\
\hline
\end{tabular}
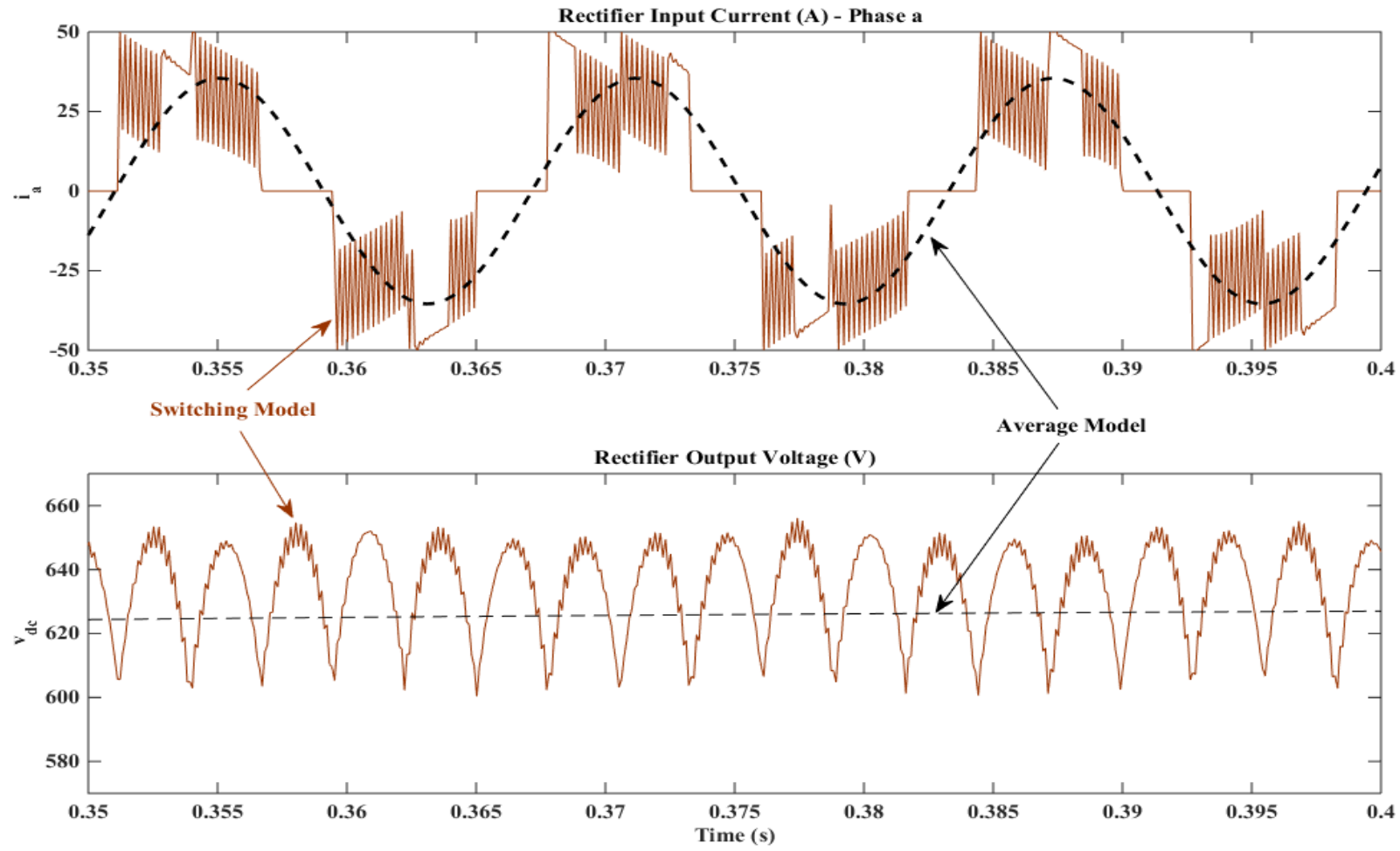

Fig. 4.29: Average model versus switching model: diode bridge rectifier waveforms. 

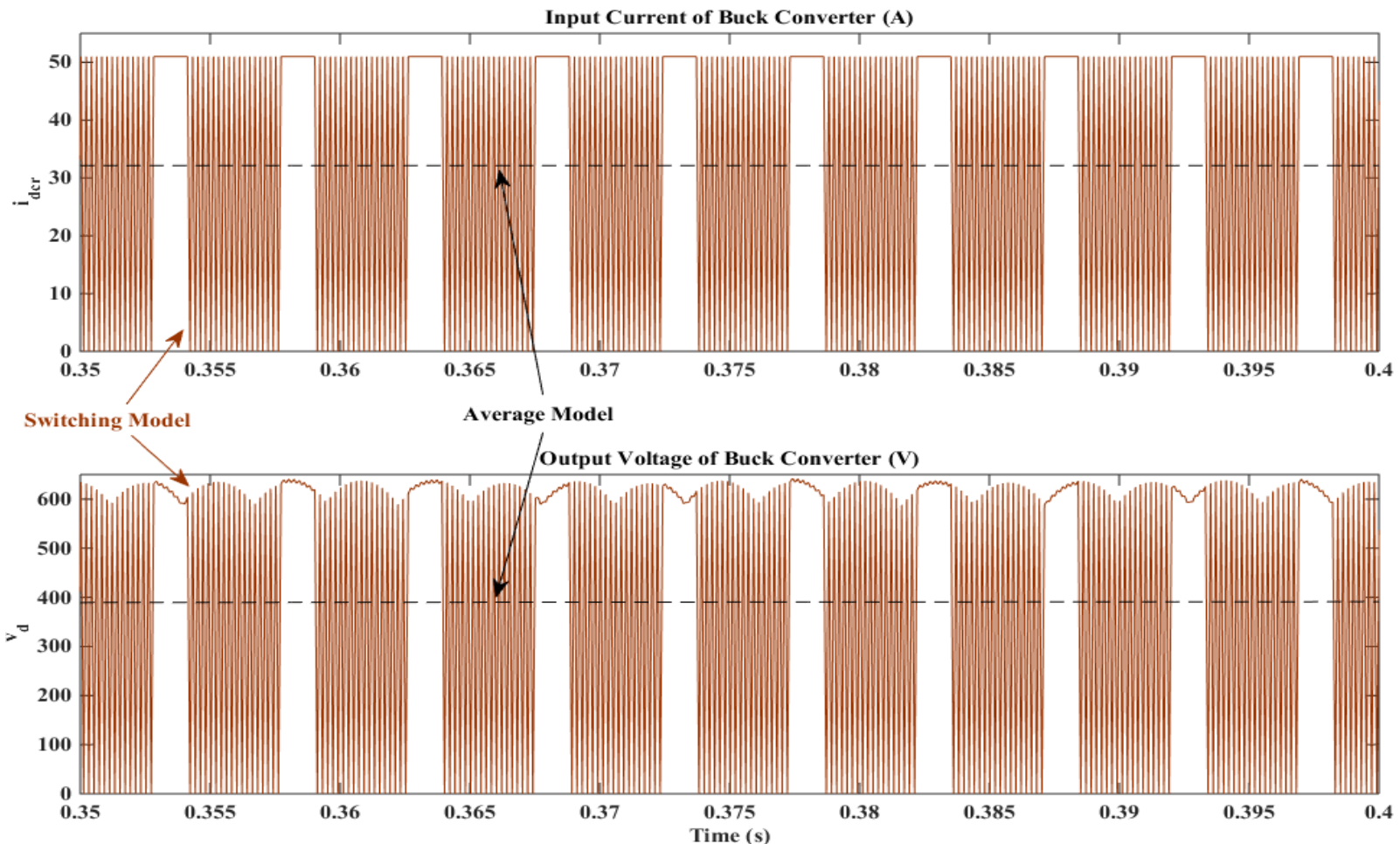

Fig. 4.30: Switching and average model waveforms of buck converter.
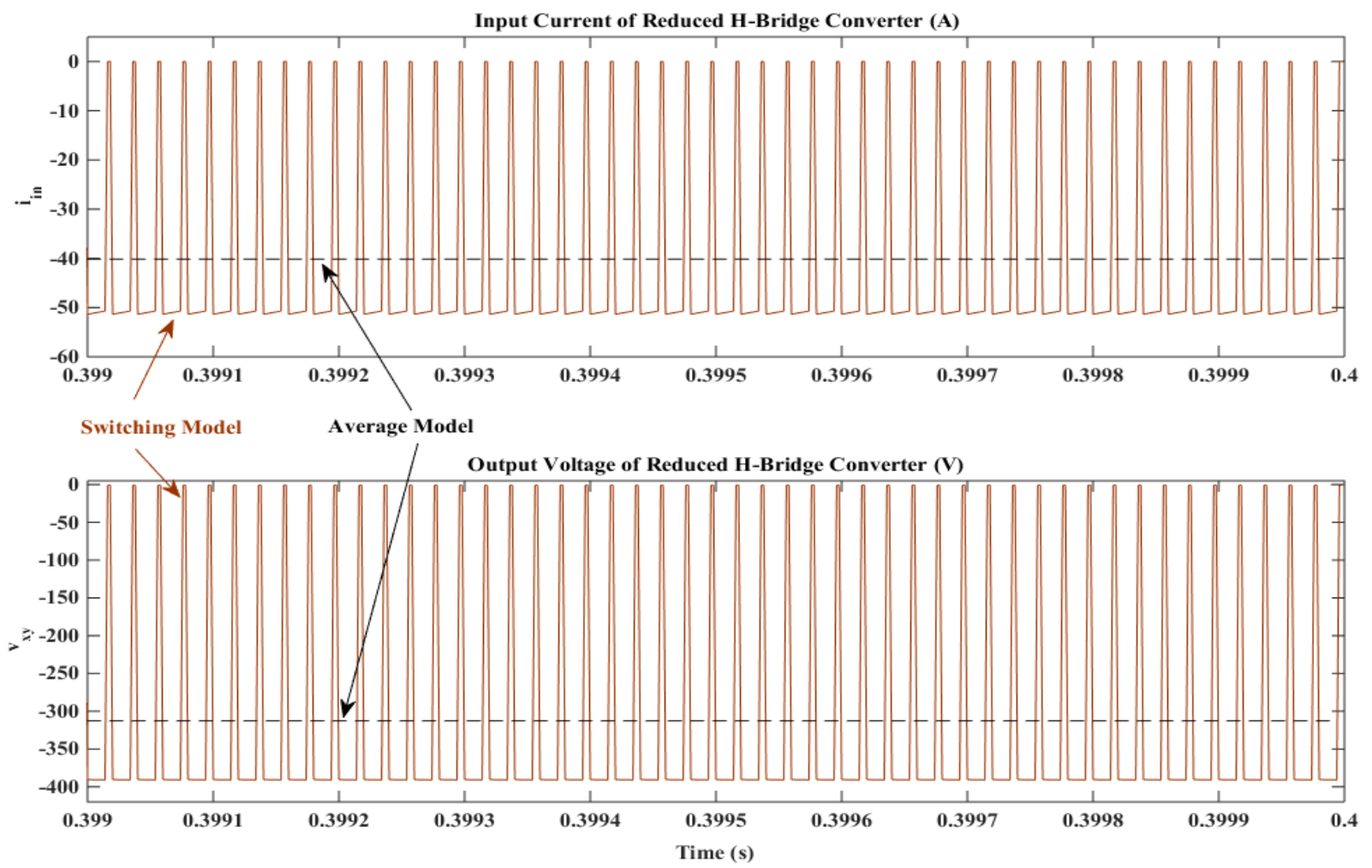

Fig. 4.31: Switching and average model waveforms of recued-H-bridge converter. 

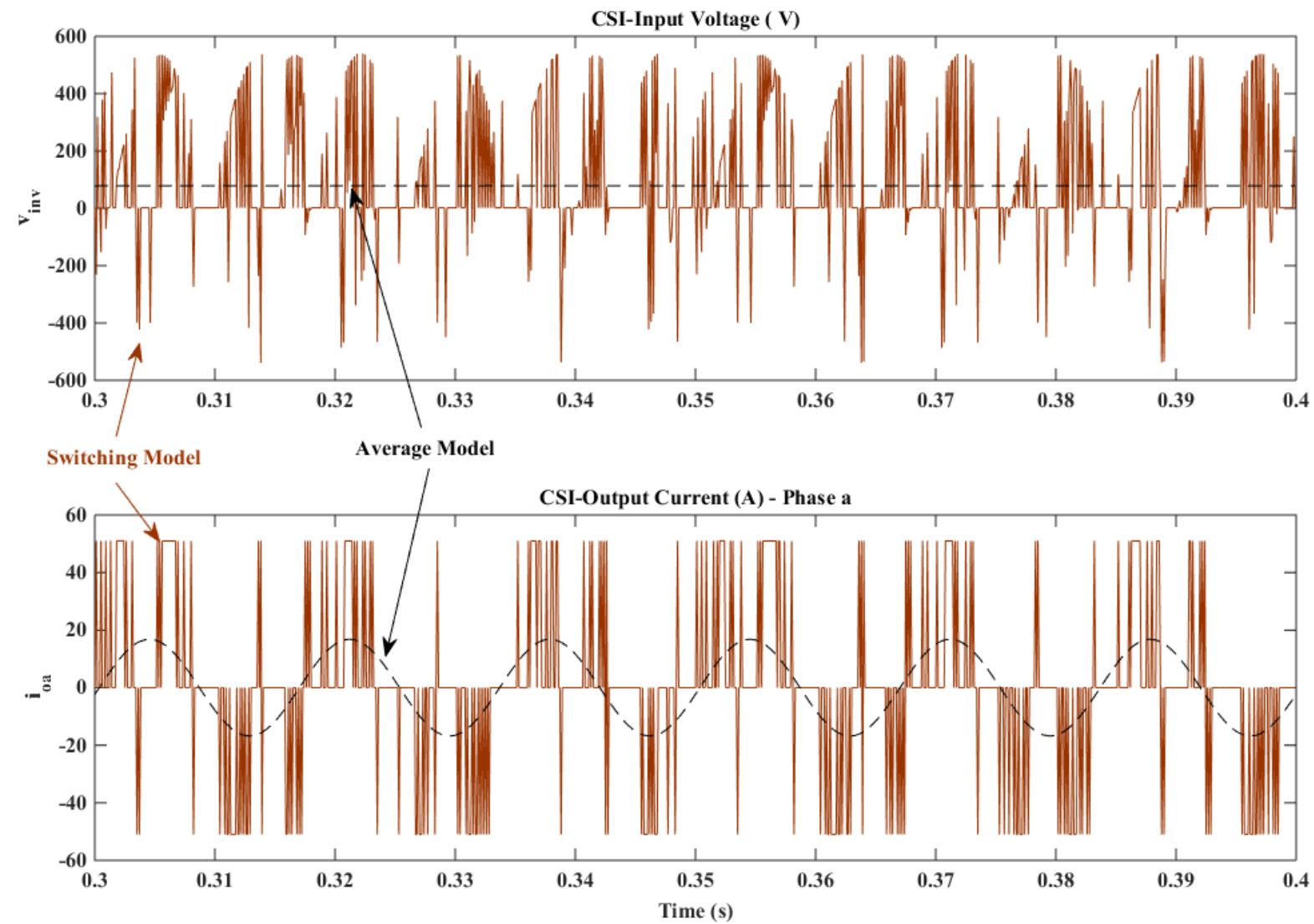

Fig. 4.32: Switching and average model waveforms of CSI.

\subsubsection{Open-Loop Step Responses of the System}

This subsection aims to investigate the dynamic behaviour of the system by simulating its step response to different input variables. This purpose is served by illustrating the following responses.

- The generator rotor speed $\left(\omega_{r}\right)$ response to step change in buck converter duty ratio $\left(d_{b}\right)$

- The generated active power $\left(P_{g}\right)$ response to step change in $d_{b}$;

- The dc-link current $\left(i_{d c}\right)$ response to step change in reduced H-bridge duty ratio $\left(d_{A}\right)$; and

- The responses of load-side $d q$ voltages $\left(v_{c i d}^{+}, v_{c i q}^{+}, v_{c i d}^{-}\right.$, and $\left.v_{c i q}^{-}\right)$to step changes in +ve seq modulation indices $\left(m_{i q}^{+}\right.$and $\left.m_{i d}^{+}\right)$. 
Although wind speed $\left(v_{w}\right)$ is an uncontrollable disturbance input, the system dynamic response to $v_{\omega}$ is important. It should be noted that the pitch angle is taken to be a stall control against wind gusts. In other words, in the system under study, pitch angle is fixed at its optimal value of zero at and below rated wind speed. Above rated wind speed, the turbine operates under passive-stall control.

Before applying any step change, the system operates at its steady-state operating point given in Table 4.6. In the following figures, a step change to a specific input is applied while the remaining inputs are maintained fixed.

The responses shown in Fig. 4.33 are related to step change in the buck converter duty ratio, $d_{b}$. The generator was running at optimum rotor speed, and hence capturing the maximum wind power at rated wind speed (i.e., $v_{\omega}, \omega_{r}$, and $P_{g}$ are all equal to $1 \mathrm{pu}$ ). At $\mathrm{t}=0.4 \mathrm{~s}$, a $50 \%$ step decrease in $d_{b}$ is applied. As shown in the figure, the rotor speed increases until it settles down to a new steady-state value (i.e., $1.2 \mathrm{pu}$ ). The generated active power oscillates for 0.1 second before it settles at $0.85 \mathrm{pu}$. Since $\omega_{r}$ deviates from its optimum value, the generated active power decreases.
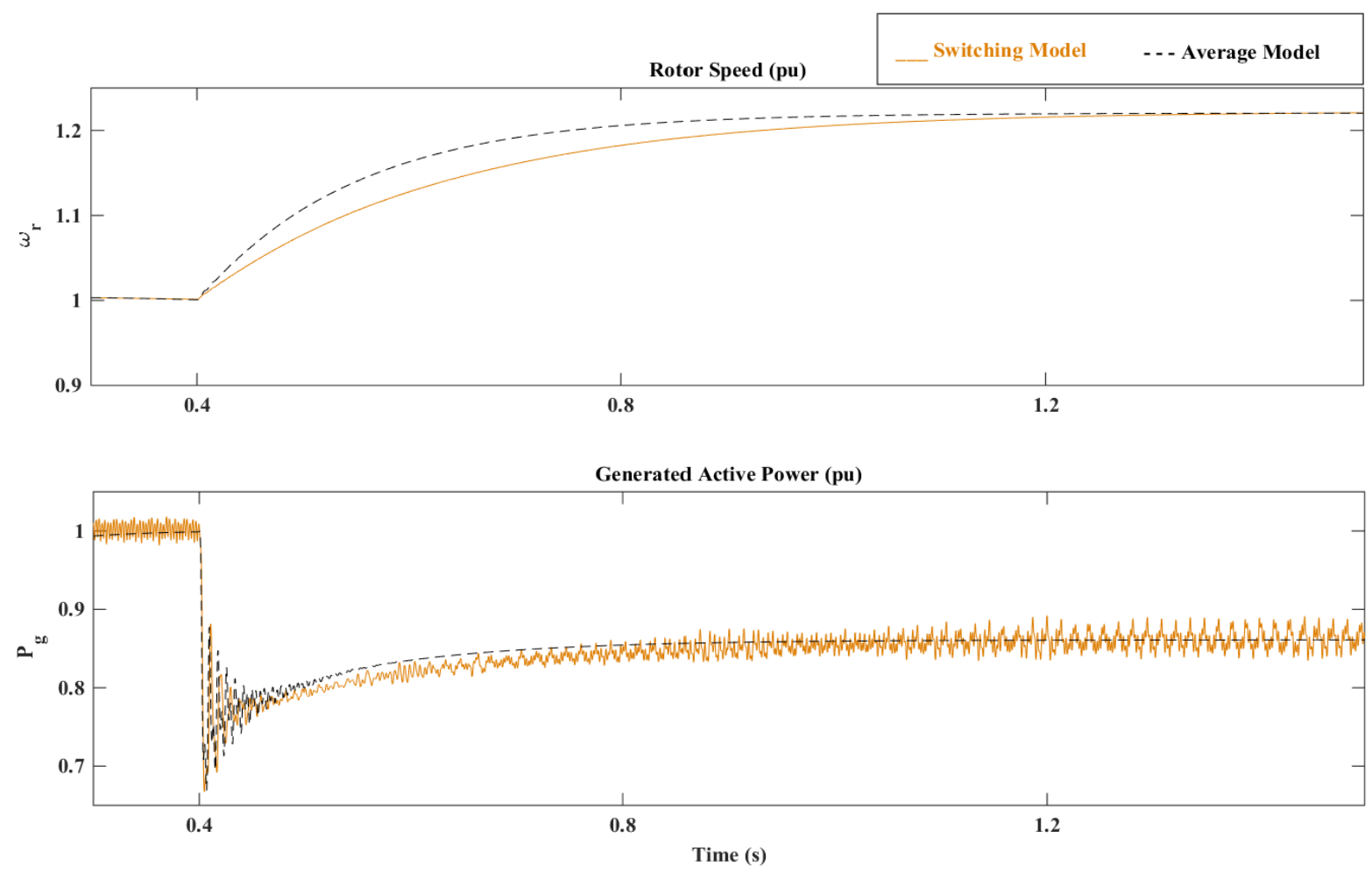

Fig. 4.33: Responses of generator rotor speed and active power to $50 \%$ step decrease in $d_{b}$. 
Wind speed $\left(v_{w}\right)$ is an uncontrollable input and the system response to a step change in wind speed shows the system performance under disturbance variation. The step responses of the generator, illustrated in Fig. 4.34, are obtained by decreasing $v_{w}$ by $20 \%$ at $\mathrm{t}=0.4 \mathrm{~s}$. As a result of reduced wind speed, less mechanical power is produced by the wind turbine. Since the active current absorbed from the generator (i.e., $d_{b} i_{d c}$ ) is kept fixed, the rotor slows down and the generated voltage is reduced in order to balance the generated electrical power with the input mechanical power. The figure shows that a rather small decrement in $v_{w}$ causes a dramatic decrease in $P_{g}$. This is because of the cubic relationship between these variables (i.e., $P_{g} \propto v_{w}^{3}$ ). As a result of significant reduction in the generated active power, the reactive power absorbed by the generator is significantly reduced.
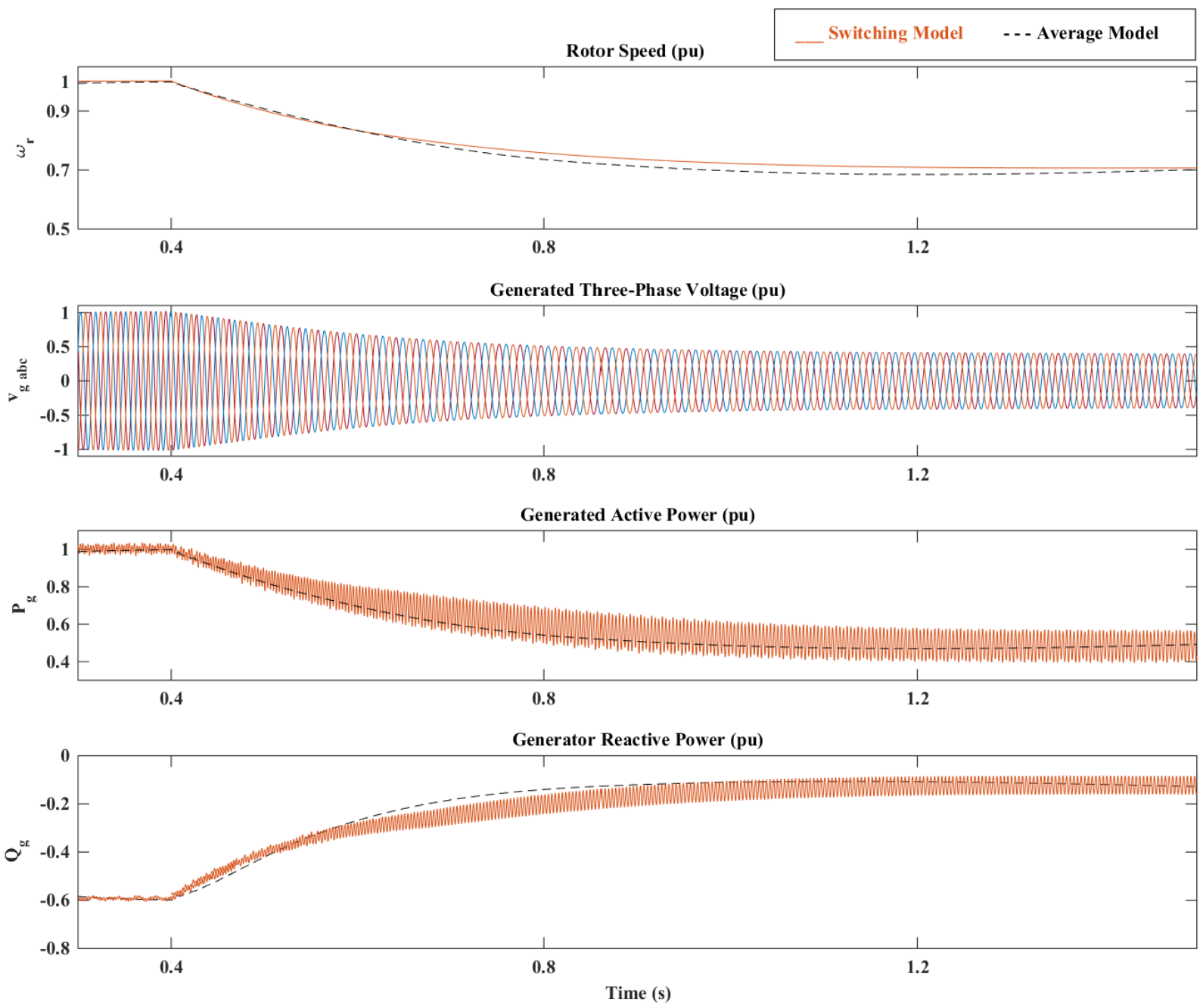

Fig. 4.34: Generator responses to $20 \%$ step decrease in $v_{W}$. 
In Fig. 4.35, an $80 \%$ step increase/decrease is applied to the duty ratio of reduced H-bridge $\left(d_{A}\right)$, at $\mathrm{t}=0.4 \mathrm{~s}$. The dc-link current steps up/down by around $28 \% / 18.5 \%$ to the new steadystate values, revealing stable operation. A smooth transition between the old and the two new steady-state conditions is noticed.

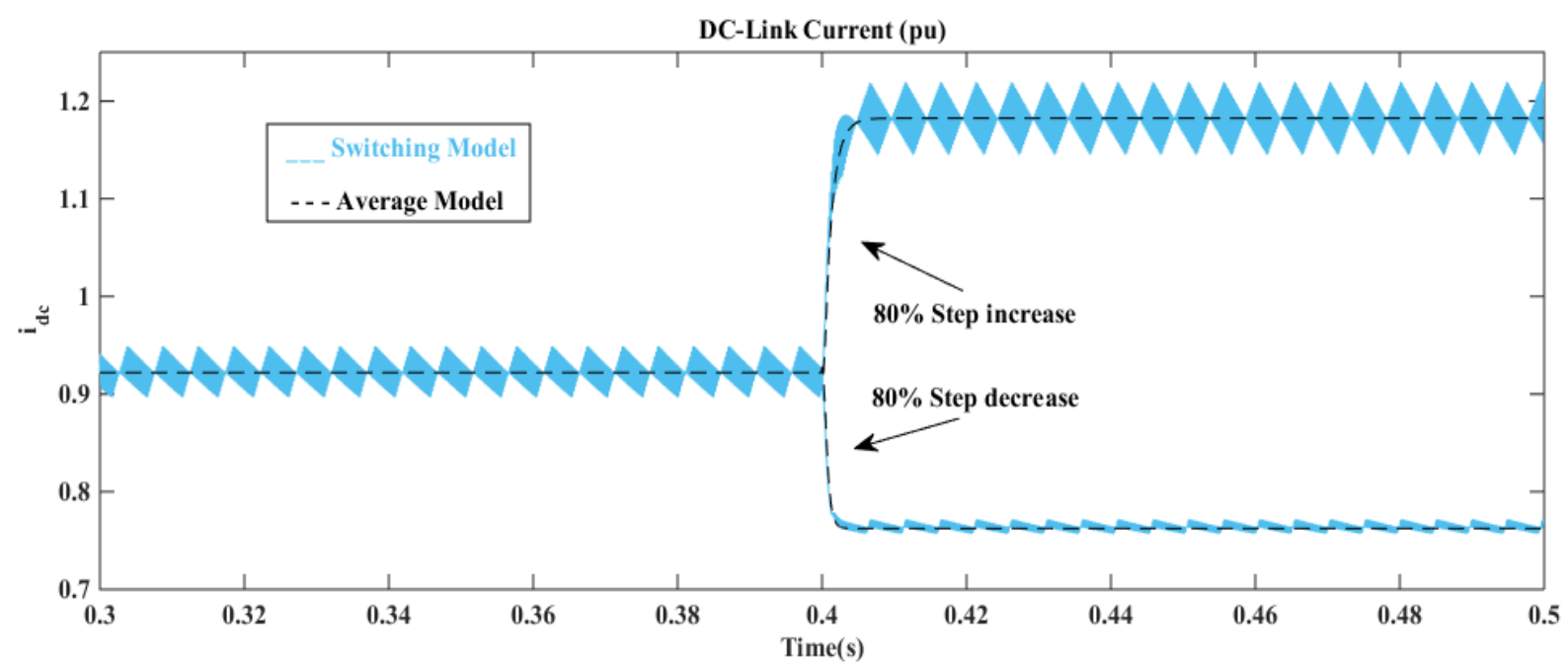

Fig. 4.35: Responses of dc-link current to $80 \%$ step change in $d_{A}$.

The responses of load-side $d q$ voltages to $50 \%$ step change in $m_{i q}^{+}$and $m_{i d}^{+}$are illustrated in Fig. 4.36 and Fig. 4.37, respectively. Before $t=0.4 \mathrm{~s}$, the system operates at its steady-state operating point given in Table 4.6. At this operating point, the load-side voltages are balanced; hence, $v_{\text {cid }}^{+}=1 \mathrm{pu}$, while $v_{\text {ciq }}^{+}=v_{\text {cid }}^{-}=v_{\text {ciq }}^{-}=0$ in synchronous $d q$ reference frame. When a step change is applied to a particular modulation index, while the remaining indices are kept fixed, voltage imbalance is introduced. As a result, load-side currents $\left(i_{p d}^{+}, i_{p q}^{+}, i_{p d}^{-}\right.$and $\left.i_{p q}^{-}\right)$ will change, leading to deviations in $v_{c i d}^{+}, v_{c i q}^{+}, v_{c i d}^{-}$and $v_{c i q}^{-}$from the desired values. In all cases, the frequency experiences transients within $\pm 0.5 \mathrm{~Hz}$ before it returns to $60 \mathrm{~Hz}$. The simulation responses shown in Fig. 4.36 and Fig. 4.37 are obtained based on the average model. The switching model responses of $d q$ voltages to $50 \%$ step increase in $m_{i d}^{+} \quad$ are given in Fig. 4.38.

The figures shown in this subsection demonstrate that with respect to the switching model, the average model doesn't represent switching harmonics, but the dynamics resulting from control system and power system interaction are preserved to a high accuracy. 

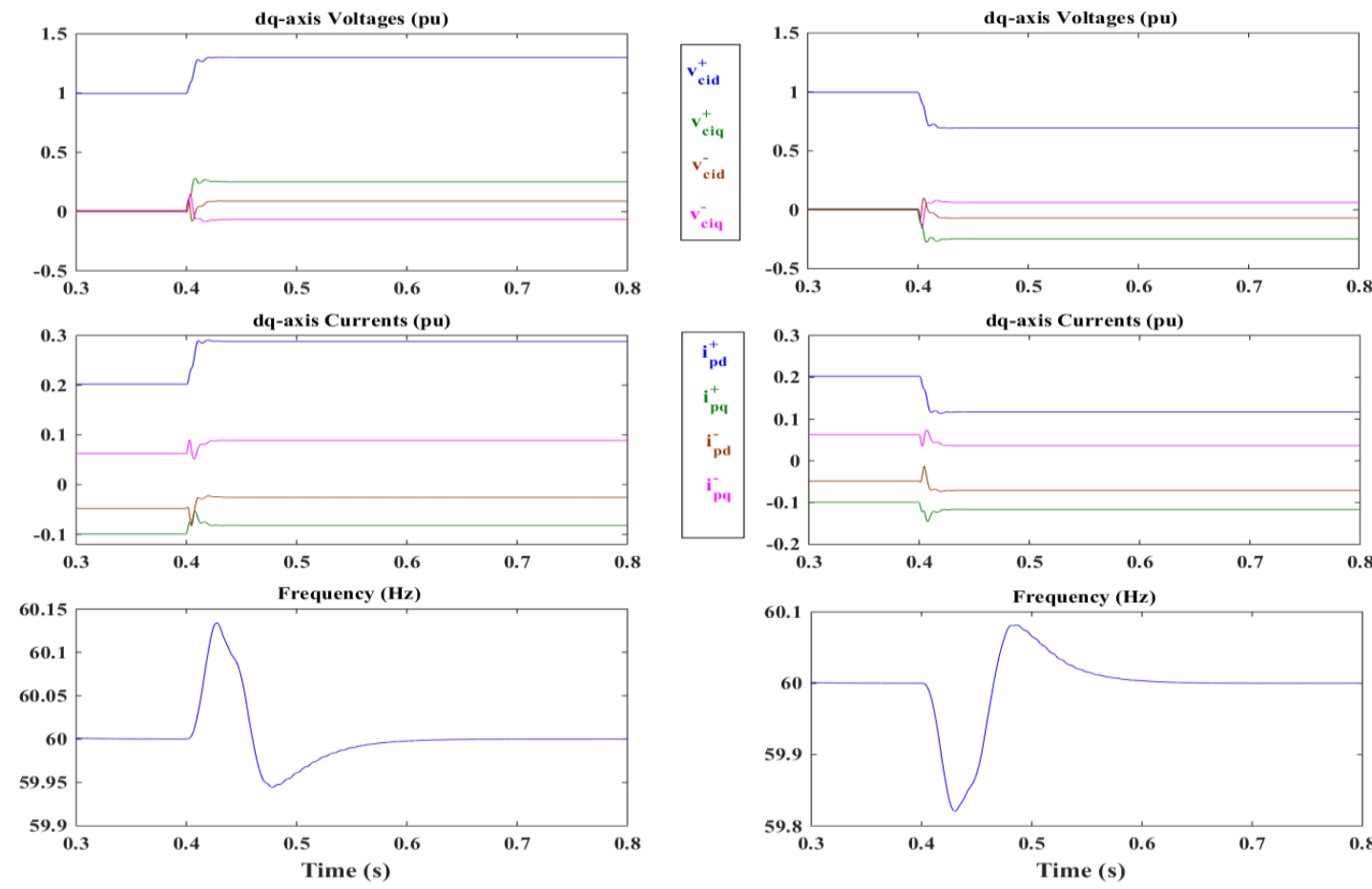

(a) $50 \%$ increase

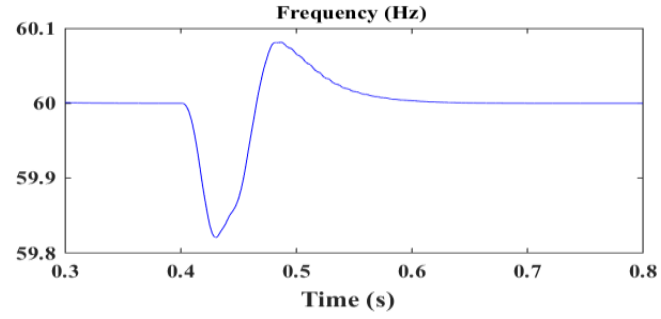

(b) $50 \%$ decrease

Fig. 4.36: Load-side responses to $50 \%$ step change in $m_{i q}^{+}$.
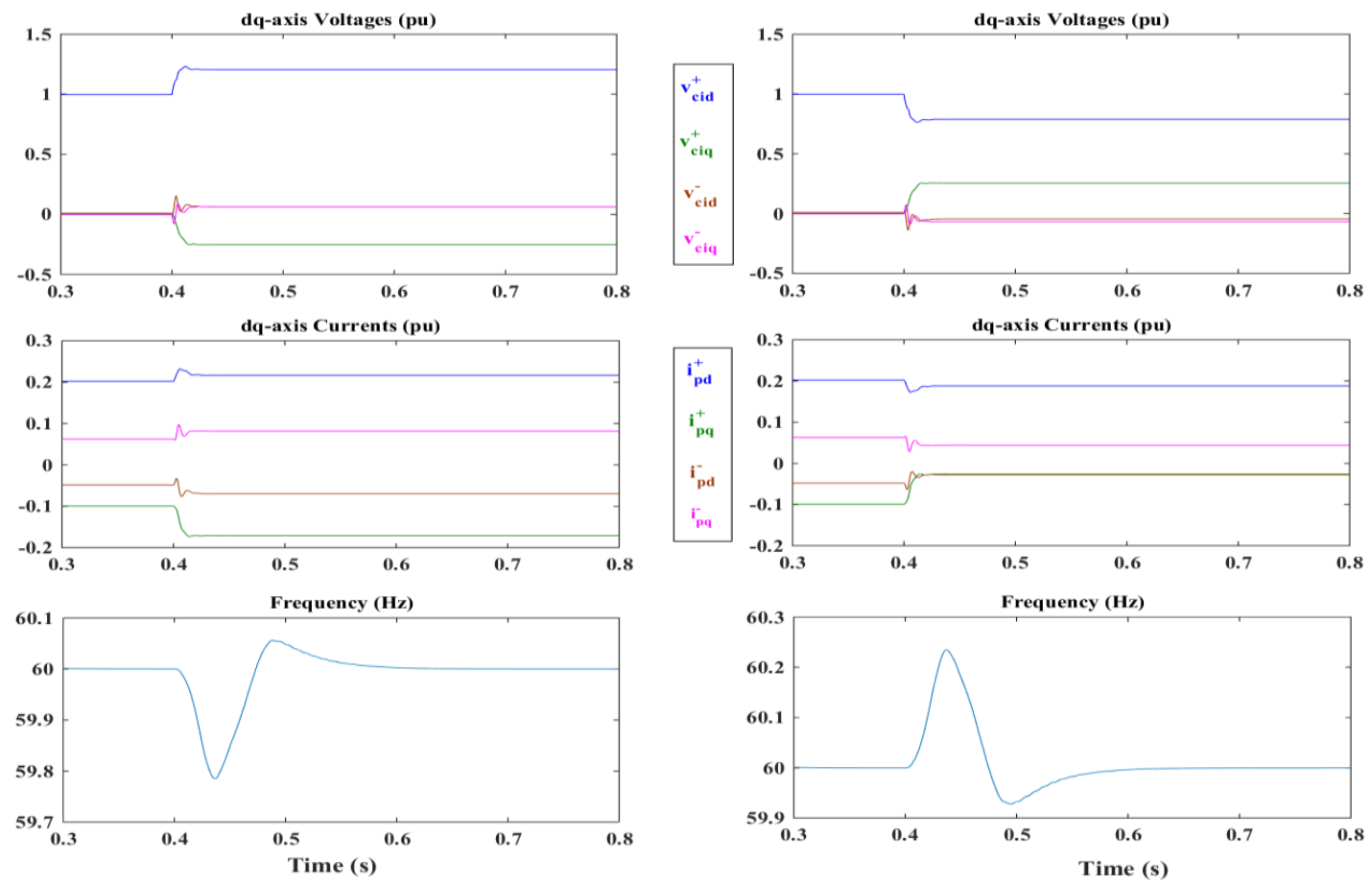

(a) $50 \%$ increase

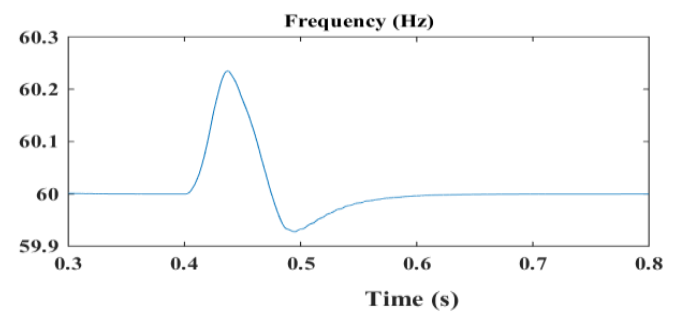

(b) $50 \%$ decrease

Fig. 4.37: Load-side responses to $50 \%$ step change in $m_{i d}^{+}$. 

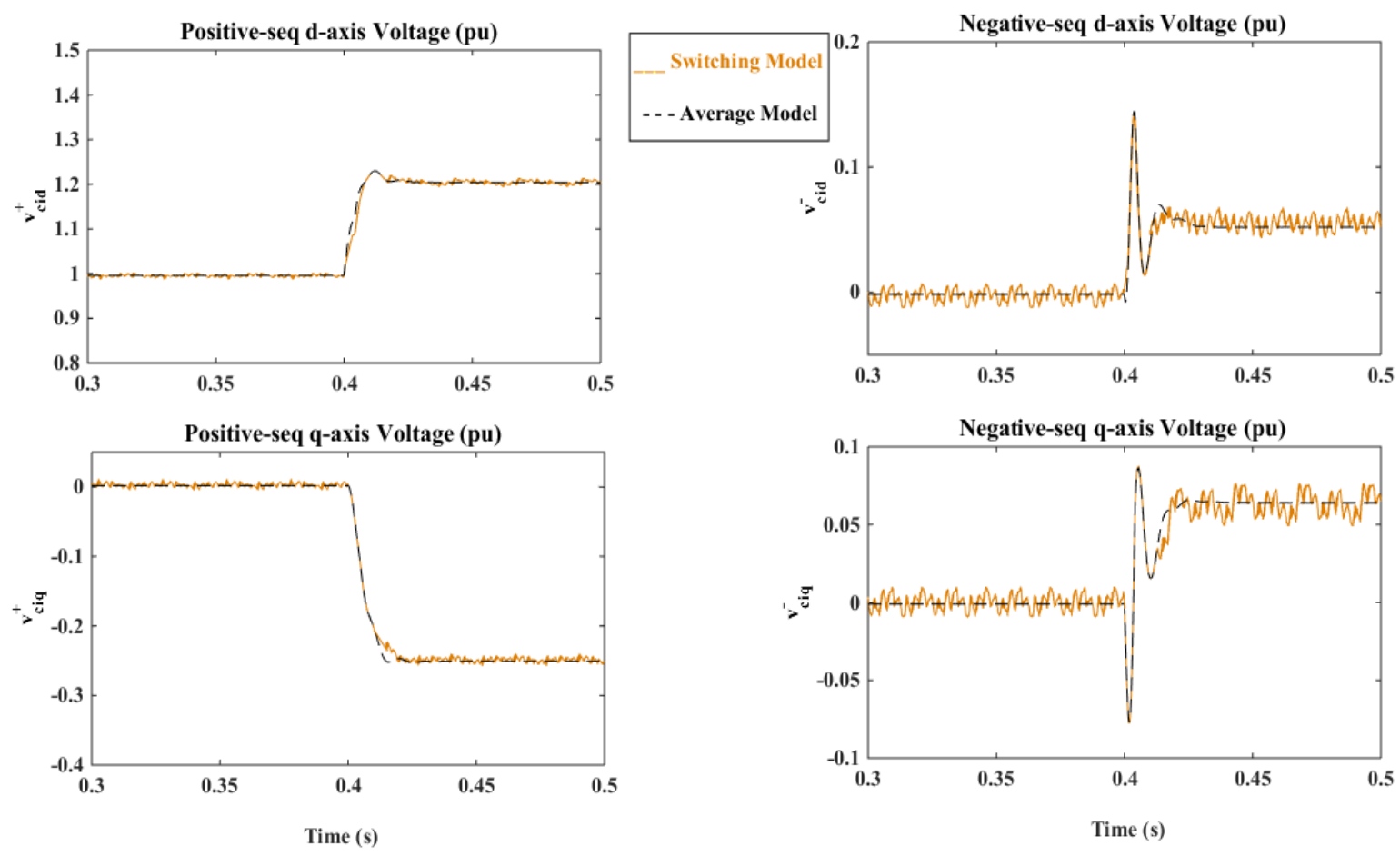

Fig. 4.38: Average and switching model load-side voltage responses to $50 \%$ step increase in $m_{i d}^{+}$.

\subsection{Small-Signal Model and Stability Analysis}

The dynamic model developed in subsection 4.11.1 and given in (4.84) consists of twenty nonlinear first-order differential equations. In order to study the stability of the system, a smallsignal model needs to be developed by linearizing the nonlinear equations around a quiescent operating point so that the system can be treated as a linear system with regard to very small disturbances. The stability analysis of the linearized system investigates its capability to return to a stable operating point after a disturbance, causing a small change in one or more of the system's state variables. It should be emphasized that a linear model derived at an operating point is valid only for small perturbations of the system around that operating point.

The small-signal model of the proposed WECS can be described by:

$$
\frac{d}{d t} \Delta X=A^{\prime} \Delta X+B^{\prime} \Delta U
$$

where 


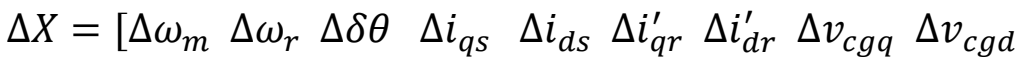

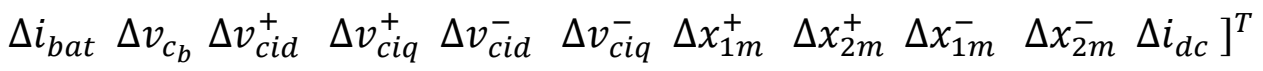

$$
\begin{aligned}
& \Delta U=\left[\begin{array}{lllllll}
\Delta v_{\omega} & \Delta d_{b} & \Delta d_{A} & \Delta m_{i d}^{+} & \Delta m_{i q}^{+} \Delta m_{i d}^{-} & \Delta m_{i q}^{-}
\end{array}\right]^{T}
\end{aligned}
$$

In (4.89), $A^{\prime}$ and $B^{\prime}$ are the Jacobian matrices, evaluated at the steady-state operating point. These matrices are provided in Appendix $E$ for WTG subsystem, ES subsystem, CSI-Load subsystem, and the overall system. The symbol $\Delta$ followed by a variable represents smallsignal perturbation of the variable. The quiescent $(\mathrm{dc})$ component of a variable at the operating point, at which the small-signal model is derived, is represented with capitalized letters. The products of small perturbations of quantities are very small, and hence can be neglected. During the process of small-signal derivation, the cross saturation effect of the SEIG is ignored and hence the mutual inductance of the machine is assumed fixed.

\subsubsection{Transfer Function and Eigenvalue Analysis}

The time-invariant linearized system described in (4.89) relates the input $\Delta U$ and the state variables $\Delta X$ in the time domain. For a particular output vector $\Delta Y$, the small-signal equation is:

$$
\Delta Y=C^{\prime} \Delta X+D^{\prime} \Delta U
$$

The direct transfer function matrix $G(s)$ from the input to the output in $s$ domain is given as

$$
G(s)=\frac{\Delta Y(s)}{\Delta U(s)}=\frac{N(s)}{D(s)}=C^{\prime}\left(s I-A^{\prime}\right)^{-1} B^{\prime}+D^{\prime}
$$

where $N(s)$ and $D(s)$ are the equivalent numerator and denominator polynomials of the transfer function, respectively. For a MIMO system, $G(s)$ is a matrix. Based on the output and input selection, different transfer functions $G_{i j}(s)$ can be obtained corresponding to the $i^{\text {th }}$ row and $j^{\text {th }}$ column of $G(s)$.

The zeros and poles of $G(s)$ are the roots of $N(s)$ and $D(s)$, respectively. The zeros of $G(s)$ change based on the output variable selection; however, the poles of $G(s)$ are associated with matrix $A^{\prime}$ only, regardless of output variable selection. The poles of the linearized system must satisfy the following equation: 


$$
\left|s I-A^{\prime}\right|=0
$$

Equation (4.94) is referred to as the characteristic equation of the system matrix $A^{\prime}$. The roots of this equation, namely the eigenvalues of matrix $A^{\prime}$, indicate the behaviour of the system at a steady-state operating point. An eigenvalue can be real or complex number. A complex or real eigenvalue corresponds to an oscillatory or a non-oscillatory mode of the system. Every pole of $G(s)$ is an eigenvalue of $A^{\prime}$. Pole locations or eigenvalues are commonly used to test the stability of the system. If every eigenvalue of $A^{\prime}$ has a negative real part, the oscillation magnitude of zero-input responses (i.e., responses driven by initial conditions) of the linearized system will decrease exponentially with time and hence the system is asymptotically stable within a small region surrounding the equilibrium point (i.e., locally stable). If one or more distinct (non-repeated) eigenvalues of $A^{\prime}$ has zero real part and the remaining eigenvalues have negative real part, the system is not asymptotically stable, but is marginally stable. On the contrary, one or more eigenvalues with positive real part indicate that the system impulse response, driven by any finite initial conditions, will blow up exponentially (i.e., increases in magnitude as time increases). Even if there is no positive eigenvalue, but there are repeated eigenvalues with zero real part, the system impulse response will still blow up, although more slowly. In both cases, the system is considered unstable. It should be noted that eigenvalues instability results hold true if no pole/zero cancellation exists. In other words, even if $A^{\prime}$ has some eigenvalues with zero or positive real part, the system may still be stable if the poles equivalent to those eigenvalues are cancelled with zeros.

Damping ratio $(\zeta)$ of an eigenvalue $\lambda=\sigma \pm j \omega$, computed as $\zeta=-\sigma / \sqrt{\left(\sigma^{2}+\omega^{2}\right)}$, is another indicator of stability. A negative $\zeta$ indicates an unstable eigenmode, while a stable eigenmode has a positive $\zeta$. If $\zeta$ of an eigenmode approaches unity, the eigenmode becomes less oscillatory and tends to be more stable. On the contrary, if $\zeta$ of an eigenmode approaches zero, the eigenmode becomes more oscillatory and tends to be less or critically stable.

\subsection{System Controllability and Observability}

Before a closed-loop control scheme is designed for a system, it must be made sure that the system is controllable. Controllability is defined as the ability to drive a system from any state to another desired state in a finite period of time. If a system state cannot be influenced by any 
of the system inputs, then the mode associated with that state is uncontrollable. If all the modes of a system are controllable, the system is called completely controllable.

Before a state estimation/measurement scheme is designed for a system, it must be confirmed that the system is observable. Observability is defined as the ability to deduce the initial state, $\Delta X(0)$, of an unforced system (i.e., $\Delta U=0$ ), by observing the output over a finite period of time. If a system state does not contribute in the system output, then the mode associated with that state is unobservable. If all the modes of a system are observable, the system is called completely observable.

In this section, controllability and observability of the linearized system are investigated based on Gramian matrices.

\subsubsection{Gramian Matrices}

Controllability and observability of a continuous, linear, time- invariant, and asymptotically stable system are determined by solving the following Lyapunov equations [144], where $W_{c}$ and $W_{o}$ are the controllability and observability Gramian matrices, respectively.

$$
\begin{aligned}
& A W_{c}+W_{c} A^{T}+B B^{T}=0 \\
& A^{T} W_{o}+W_{o} A+C^{T} C=0
\end{aligned}
$$

The system is controllable and observable if $W_{c}$ and $W_{o}$ are full rank matrices, which can be checked by applying singular value decomposition (SVD) [144]. SVD of $W_{c}$ and $W_{o}$ at an operating point can be used to indicate system controllability and observability, respectively. If all SVDs of $W_{c}$ have non-zero values, the system is completely controllable. Similarly, if all SVDs of $W_{o}$ have non-zero values, the system is completely observable. The size of the numerical error which is likely introduced by matrix computation can be found from the condition number calculated by dividing the maximum SVD over minimum SVD of the matrix. While a small condition number indicates a well-conditioned system, a large condition number indicates ill-conditioned system.

Even if matrix $A$ has a positive $\lambda$, the Lyapunov equations (4.95) and (4.96) may still have solutions. In fact, they have unique solutions if and only if $\lambda(A)+\lambda(\bar{A}) \neq 0$, where $\bar{A}$ is a matrix with entries equal to complex conjugates of $A[145]$. 


\subsection{Linearized Model Evaluation}

In order to investigate stability, controllability and observability of the system represented by the developed mathematical model, a $20 \mathrm{~kW}$ wind turbine system is considered. The rating and parameters of the studied turbine are given in Appendix A (Table A.2). For linearization, three operating points are selected based on the operating mode of the energy storage subsystem. The operating points are defined as follows:

\section{a) Operating point 1(o.p.1): Charging Mode}

At this operating point, the system is operated under rated wind speed (i.e., $P_{g, o p t}=$ $20 \mathrm{~kW}$ ) and $20 \%$ rated load. Therefore, $20 \%$ of the generated power under MPPT $\left(P_{g, o p t}\right)$ is supplied to the load (i.e., $\left.P_{L}=4 \mathrm{~kW}\right)$ and the remaining $80 \%$ of $P_{g, o p t}$ is stored in the storage battery (i.e., $P_{b a t}=-16 \mathrm{~kW}$ ).

\section{b) Operating point 2 (o.p.2): Freewheeling mode}

At this operating point, the system is operated under rated wind speed (i.e., $P_{g, o p t}=$ $20 \mathrm{~kW}$ ) and rated load condition (i.e., $P_{L}=20 \mathrm{~kW}$ ). Therefore, $P_{g, o p t}$ is totally transferred to the load (assuming a lossless system) and the average power absorbed or delivered by the storage battery is equal to zero.

c) Operating point 3(o.p.3): Discharging mode.

At this operating point, the system is operated under $66.7 \%$ rated wind speed (i.e., $29.7 \%$ of rated generated power or $P_{g, o p t}=5.94 \mathrm{~kW}$ ) and $50 \%$ load (i.e., $P_{L}=$ $10 \mathrm{~kW})$. The demand is higher than $P_{g, o p t}$ and hence the storage battery compensates for the shortage (i.e., $P_{b a t}=4.06 \mathrm{~kW}$ ).

The three operating points selected for linearization are given in Table 4.7. As mentioned in section 4.13, all variables are represented by capitalized letters to indicate quiescent (dc) component of the variable at the selected operating point for linearization.

Based on the analysis described in previous sections, the eigenvalues, damping ratios, and SVDs of Gramian controllability/observability matrices of the small-signal model linearized 
around each of the three defined operating points are given in Table 4.8, Table 4.9, Table 4.10, and Table 4.11.

Table 4.7: Input, state and output variables at the operating points for linearization.

\begin{tabular}{|c|c|c|c|}
\hline \multicolumn{4}{|c|}{ Operating point 1: Rated wind speed and 20\% load (Charging Mode) } \\
\hline$V_{\omega}=12 \mathrm{~m} / \mathrm{s}$ & $D_{b}=0.63$ & $D_{A}=0.108$ & $M_{i d}^{+}=0.1945$ \\
\hline$M_{i q}^{+}=0.233$ & $M_{i d}^{-}=-0.049$ & $M_{i q}^{-}=0.059$ & $I_{d c}=51 \mathrm{~A}$ \\
\hline$W_{m}=1.518 \mathrm{rad} / \mathrm{s}$ & $W_{r}=379.5 \mathrm{rad} / \mathrm{s}$ & $\delta \theta=3 \mathrm{rad}$ & $I_{q s}=-19.8 \mathrm{~A}$ \\
\hline$I_{d s}=-35.5 \mathrm{~A}$ & $I_{q r}^{\prime}=4.4 \mathrm{~A}$ & $I_{d r}^{\prime}=37.5 \mathrm{~A}$ & $V_{c g q}=0$ \\
\hline$V_{c g d}=375.6 \mathrm{~V}$ & $I_{b a t}=-40 \mathrm{~A}$ & $V_{c_{b}}=393.3 \mathrm{~V}$ & SoC $=50 \%$ \\
\hline$V_{c i d}^{+}=310.3 \mathrm{~V}$ & $V_{c i q}^{+}=0$ & $V_{\text {cid }}^{-}=0$ & $V_{c i q}^{-}=0$ \\
\hline $\begin{array}{l}I_{p d}^{+}=42.97 / 5 A \\
Y_{P}^{+}=0.1385 / 5\end{array}$ & $\begin{array}{c}I_{p q}^{+}=-21.47 / 5 A \\
Y_{Q}^{+}=0.0692 / 5\end{array}$ & $\begin{aligned} I_{p d}^{-} & =-10.92 / 5 A \\
Y_{Q_{p d}}^{-} & =-0.0352 / 5\end{aligned}$ & $\begin{aligned} I_{p q}^{-} & =12.97 / 5 A \\
Y_{Q_{p q}}^{-} & =-0.0418 / 5\end{aligned}$ \\
\hline \multicolumn{4}{|c|}{ Operating point 2: Rated wind speed and rated load (Freewheeling mode) } \\
\hline$V_{\omega}=12 \mathrm{~m} / \mathrm{s}$ & $D_{b}=0.58$ & $D_{A}=0.5$ & $M_{i d}^{+}=0.894$ \\
\hline$M_{i q}^{+}=-0.143$ & $M_{i d}^{-}=-0.227$ & $M_{i q}^{-}=0.270$ & $I_{d c}=55.5 \mathrm{~A}$ \\
\hline$W_{m}=1.518 \mathrm{rad} / \mathrm{s}$ & $W_{r}=379.5 \mathrm{rad} / \mathrm{s}$ & $\delta \theta=3 \mathrm{rad}$ & $I_{q s}=-19.8 \mathrm{~A}$ \\
\hline$I_{d s}=-35.5 \mathrm{~A}$ & $I_{q r}^{\prime}=4.4 A$ & $I_{d r}^{\prime}=37.5 \mathrm{~A}$ & $V_{c g q}=0$ \\
\hline$V_{c g d}=375.6 \mathrm{~V}$ & $I_{b a t}=0$ & $V_{c_{b}}=395.5 \mathrm{~V}$ & SoC $=60 \%$ \\
\hline$V_{c i d}^{+}=310.3 \mathrm{~V}$ & $V_{c i q}^{+}=0$ & $V_{\text {cid }}^{-}=0$ & $V_{\text {ciq }}^{-}=0$ \\
\hline $\begin{array}{c}I_{p d}^{+}=42.97 \mathrm{~A} \\
Y_{P}^{+}=0.1385\end{array}$ & $\begin{array}{c}I_{p q}^{+}=-21.47 A \\
Y_{Q}^{+}=0.0692\end{array}$ & $\begin{array}{c}I_{p d}^{-}=-10.92 A \\
Y_{Q_{p d}}^{-}=-0.0352\end{array}$ & $\begin{array}{c}I_{p q}^{-}=12.97 \mathrm{~A} \\
Y_{Q_{p q}}^{-}=-0.0418\end{array}$ \\
\hline \multicolumn{4}{|c|}{ Operating point 3: $66.7 \%$ of rated wind speed and $50 \%$ load (Discharging mode) } \\
\hline$V_{\omega}=8 \mathrm{~m} / \mathrm{s}$ & $D_{b}=0.82$ & $D_{A}=0.69$ & $M_{i d}^{+}=0.894$ \\
\hline$M_{i q}^{+}=0.161$ & $M_{i d}^{-}=-0.227$ & $M_{i q}^{-}=0.270$ & $I_{d c}=27.75 \mathrm{~A}$ \\
\hline$W_{m}=1.012 \mathrm{rad} / \mathrm{s}$ & $W_{r}=253 \mathrm{rad} / \mathrm{s}$ & $\delta \theta=1.33 \mathrm{rad}$ & $I_{q s}=-12 A$ \\
\hline$I_{d s}=-25 A$ & $I_{q r}^{\prime}=3.3 \mathrm{~A}$ & $I_{d r}^{\prime}=25.6 \mathrm{~A}$ & $V_{c g q}=0$ \\
\hline$V_{c g d}=158 \mathrm{~V}$ & $I_{\text {bat }}=10.5 \mathrm{~A}$ & $V_{c_{b}}=388 \mathrm{~V}$ & $S o C=40 \%$ \\
\hline$V_{\text {cid }}^{+}=310.3 \mathrm{~V}$ & $V_{c i q}^{+}=0$ & $V_{\text {cid }}^{-}=0$ & $V_{\text {ciq }}^{-}=0$ \\
\hline $\begin{array}{l}I_{p d}^{+}=42.97 / 2 A \\
Y_{P}^{+}=0.1385 / 2\end{array}$ & $\begin{array}{c}I_{p q}^{+}=-21.47 / 2 A \\
Y_{Q}^{+}=0.0692 / 2\end{array}$ & $\begin{array}{c}I_{p d}^{-}=-10.92 / 2 A \\
Y_{Q_{p d}}^{-}=-0.0352 / 2\end{array}$ & $\begin{array}{c}I_{p q}^{-}=12.97 / 2 \mathrm{~A} \\
Y_{Q_{p q}}^{-}=-0.0418 / 2\end{array}$ \\
\hline
\end{tabular}


Table 4.8: Eigenvalues and damping ratios of the linearized system at o.p.1 and o.p.2.

\begin{tabular}{|c|c|c|c|}
\hline \multicolumn{2}{|c|}{ Operating point 1 } & \multicolumn{2}{c|}{ Operating point 2 } \\
\hline Eigenvalue & Damping ratio & Eigenvalue & Damping ratio \\
\hline$\lambda_{1}=-9731$ & 1.0 & $\lambda_{1}=-9721$ & 1.0 \\
\hline$\lambda_{2}=-4208$ & 1.0 & $\lambda_{2}=-4243$ & 1.0 \\
\hline$\lambda_{3,4}=-47.27 \pm j 1854$ & 0.0255 & $\lambda_{3,4}=-47.3 \pm j 1854$ & 0.0255 \\
\hline$\lambda_{5,6}=-44.27 \pm j 1478$ & 0.0299 & $\lambda_{5,6}=-44.33 \pm j 1478$ & 0.0299 \\
\hline$\lambda_{7,8}=-103 \pm j 191$ & 0.4746 & $\lambda_{7,8}=-100 \pm j 188$ & 0.4696 \\
\hline$\lambda_{9,10}=-100 \pm j 75$ & 0.8 & $\lambda_{9,10}=-100 \pm j 75$ & 0.8 \\
\hline$\lambda_{11}=-113$ & 1.0 & $\lambda_{11}=-114$ & 1.0 \\
\hline$\lambda_{12,13}=-111 \pm j 111$ & 0.7071 & $\lambda_{12,13}=-111 \pm j 111$ & 0.7071 \\
\hline$\lambda_{14}=-23.22$ & 1.0 & $\lambda_{14}=-23.14$ & 1.0 \\
\hline$\lambda_{15}=-20.51$ & 1.0 & $\lambda_{15}=-20.53$ & 1.0 \\
\hline$\lambda_{16}=-0.016$ & 1.0 & $\lambda_{16}=-0.016$ & 1.0 \\
\hline$\lambda_{17}=-0.016$ & 1.0 & $\lambda_{17}=-0.016$ & 1.0 \\
\hline$\lambda_{18}=-0.0005$ & 1.0 & $\lambda_{18}=-0.0011$ & 1.0 \\
\hline$\lambda_{19}=-4.69 \times 10^{-9}$ & 1.0 & $\lambda_{19}=1.3 \times 10^{-6}$ & -1.0 \\
\hline$\lambda_{20}=-7.32 \times 10^{-10}$ & 1.0 & $\lambda_{20}=-4.15 \times 10^{-9}$ & 1.0 \\
\hline
\end{tabular}

Table 4.9: Singular values of Gramian matrices at o.p.1 and o.p.2.

\begin{tabular}{|l|c|c|c|c|}
\hline & \multicolumn{2}{|c|}{ Operating point 1 } & \multicolumn{2}{c|}{ Operating point 2 } \\
\hline & $W_{c}$ & $W_{o}$ & $W_{c}$ & $W_{o}$ \\
\hline Singular values of & $1.361 \times 10^{23}$ & $1.054 \times 10^{17}$ & $1.391 \times 10^{23}$ & $1.049 \times 10^{17}$ \\
the matrix & $1.168 \times 10^{17}$ & $2.119 \times 10^{14}$ & $7.385 \times 10^{22}$ & $2.352 \times 10^{16}$ \\
& $3.785 \times 10^{16}$ & $7.758 \times 10^{8}$ & $4.006 \times 10^{19}$ & $1.438 \times 10^{12}$ \\
& $1.175 \times 10^{13}$ & $1.029 \times 10^{8}$ & $8.185 \times 10^{18}$ & $9.469 \times 10^{9}$ \\
& $1.139 \times 10^{13}$ & $3.400 \times 10^{6}$ & $1.717 \times 10^{16}$ & $2.117 \times 10^{7}$ \\
& $3.309 \times 10^{9}$ & $3.276 \times 10^{2}$ & $7.447 \times 10^{13}$ & $3.400 \times 10^{6}$ \\
& $1.027 \times 10^{9}$ & $1.610 \times 10^{2}$ & $5.555 \times 10^{13}$ & $2.492 \times 10^{5}$ \\
& $4.082 \times 10^{6}$ & $1.031 \times 10^{2}$ & $1.383 \times 10^{13}$ & $1.312 \times 10^{2}$ \\
& $5.388 \times 10^{5}$ & $2.997 \times 10^{1}$ & $1.294 \times 10^{13}$ & $5.719 \times 10^{1}$ \\
& $1.644 \times 10^{5}$ & $2.613 \times 10^{1}$ & $1.572 \times 10^{12}$ & $2.083 \times 10^{1}$ \\
& $3.118 \times 10^{4}$ & $2.070 \times 10^{1}$ & $1.563 \times 10^{9}$ & 8.145 \\
& $3.842 \times 10^{3}$ & 4.643 & $4.198 \times 10^{7}$ & 1.121 \\
& $1.876 \times 10^{3}$ & 3.148 & $3.723 \times 10^{7}$ & $5.715 \times 10^{-1}$ \\
& $1.122 \times 10^{3}$ & 2.268 & $3.389 \times 10^{7}$ & $1.213 \times 10^{-1}$ \\
& $9.989 \times 10^{2}$ & 1.136 & $1.521 \times 10^{7}$ & $9.223 \times 10^{-2}$ \\
& $1.695 \times 10^{2}$ & $1.804 \times 10^{-1}$ & $5.142 \times 10^{6}$ & $3.061 \times 10^{-2}$ \\
& $1.221 \times 10^{2}$ & $7.482 \times 10^{-2}$ & $2.896 \times 10^{5}$ & $1.061 \times 10^{-2}$ \\
& $6.672 \times 10^{1}$ & $1.080 \times 10^{-3}$ & $2.310 \times 10^{4}$ & $8.412 \times 10^{-4}$ \\
& $3.595 \times 10^{1}$ & $5.617 \times 10^{-4}$ & $2.239 \times 10^{3}$ & 0 \\
& $4.711 \times 10^{-1}$ & $2.233 \times 10^{-5}$ & $9.611 \times 10^{2}$ & 0 \\
\hline Rank of the matrix & 20 & 20 & & 20 \\
& Completely & Completely & Completely & Not Completely \\
& Controllable & observable & Controllable & observable \\
\hline & $2.889 \times 10^{23}$ & $4.720 \times 10^{21}$ & $1.447 \times 10^{20}$ & - \\
\hline
\end{tabular}


Table 4.10: Eigenvalues and damping ratios of the linearized system at o.p.3.

\begin{tabular}{|c|c|}
\hline Eigenvalue & Damping ratio \\
\hline$\lambda_{1}=-9731$ & 1.0 \\
\hline$\lambda_{2}=-4208$ & 1.0 \\
\hline$\lambda_{3,4}=-50.15 \pm j 1468$ & 0.0341 \\
\hline$\lambda_{5,6}=-42.96 \pm j 1091$ & 0.0393 \\
\hline$\lambda_{7,8}=-79.73 \pm j 170$ & 0.4246 \\
\hline$\lambda_{9,10}=-100 \pm j 75$ & 0.8 \\
\hline$\lambda_{11}=-118$ & 1.0 \\
\hline$\lambda_{12,13}=-111 \pm j 111$ & 0.7071 \\
\hline$\lambda_{14}=-22.98$ & 1.0 \\
\hline$\lambda_{15}=-3.66$ & 1.0 \\
\hline$\lambda_{16}=-0.016$ & 1.0 \\
\hline$\lambda_{17}=-0.016$ & 1.0 \\
\hline$\lambda_{18}=-2.51 \times 10^{-4}$ & 1.0 \\
\hline$\lambda_{19}=-2.368 \times 10^{-8}$ & 1.0 \\
\hline$\lambda_{20}=-7.61 \times 10^{-9}$ & 1.0 \\
\hline & \\
\hline
\end{tabular}

Table 4.11: Singular values of Gramian matrices at o.p.3.

\begin{tabular}{|c|c|c|}
\hline & $W_{c}$ & $W_{o}$ \\
\hline \multirow{3}{*}{ Singular values of the } & $7.777 \times 10^{22}$ & $2.102 \times 10^{17}$ \\
matrix & $5.537 \times 10^{15}$ & $8.833 \times 10^{12}$ \\
& $1.946 \times 10^{15}$ & $1.107 \times 10^{10}$ \\
& $1.276 \times 10^{13}$ & $3.730 \times 10^{9}$ \\
& $7.331 \times 10^{12}$ & $3.400 \times 10^{6}$ \\
& $3.603 \times 10^{12}$ & $1.608 \times 10^{3}$ \\
& $3.546 \times 10^{12}$ & $2.500 \times 10^{1}$ \\
& $6.150 \times 10^{8}$ & $2.259 \times 10^{1}$ \\
& $3.124 \times 10^{7}$ & 9.568 \\
& $1.119 \times 10^{7}$ & 7.199 \\
& $8.758 \times 10^{6}$ & $8.697 \times 10^{-1}$ \\
& $2.785 \times 10^{6}$ & $1.112 \times 10^{-1}$ \\
& $1.585 \times 10^{6}$ & $5.103 \times 10^{-2}$ \\
& $9.929 \times 10^{5}$ & $4.909 \times 10^{-2}$ \\
& $3.564 \times 10^{5}$ & $3.804 \times 10^{-3}$ \\
& $1.881 \times 10^{4}$ & $2.173 \times 10^{-3}$ \\
& $1.938 \times 10^{3}$ & $1.098 \times 10^{-3}$ \\
& $2.922 \times 10^{2}$ & $1.797 \times 10^{-4}$ \\
& $2.561 \times 10^{-1}$ & $1.750 \times 10^{-4}$ \\
& 20 & $1.177 \times 10^{-5}$ \\
\hline Rank of the matrix & Completely Controllable & Completely observable \\
\hline Condition no. & $3.036 \times 10^{23}$ & $1.785 \times 10^{22}$ \\
\hline
\end{tabular}


Based on the results obtained in Table 4.8, Table 4.9, Table 4.10, and Table 4.11, the stability, controllability, and observability of the system at the three operating points, defined in Table 4.7, are evaluated as follows:

\section{- Stability}

- Every eigenvalue of the small-signal model linearized at o.p.1 and o.p.3 has negative real part and hence the linearized system is asymptotically stable at these two operating points. However, eigenmodes corresponding to $\lambda_{3,4}$ and $\lambda_{5,6}$ are very oscillatory modes with very small damping ratios (close to zero) and the system tends to be critically stable. Moreover, eigenvalues $\lambda_{16}$ to $\lambda_{20}$ result in a large settling time and a slow open-loop system. The speed of responses as well as stability margins are to be taken care of in the closed loop control design.

- The real eigenvalue $\lambda_{19}$ is positive at o.p.2; thus, its corresponding eigenmode will blow up exponentially, although very slowly, as $\lambda_{19}$ is almost at the origin. This indicates an unstable open loop system around o.p.2. It is the function of closed loop controller to stabilize the system at this operating point.

\section{- Controllability}

The singular values of controllability matrix $W_{c}$ under the three operating points have non-zero values; therefore, the system is completely controllable. However, the singular values of $W_{c}$ are distributed over a wide range, the indicator of an illconditioned system close to rank deficiency. This is shown by the large condition number of $W_{c}$ for each of the three operating points.

\section{- Observability}

The singular values of observability matrix $W_{o}$ under o.p.1 and o.p.3 have non-zero values; therefore, the system is completely observable. However, some of the SVDs are too small (almost zero), the indicator of hardly-observable modes that almost don't contribute to any of the desired outputs. At o.p.2, $W_{o}$ has two zero SVDs; hence, the system is not completely observable. During freewheeling mode of operation (o.p.2), the average power exchange by the storage subsystem is zero. In 
other words, the energy storage subsystem doesn't participate in the system performance at this operating point. Therefore, the eigenmodes associated with battery-side LC filter, namely, $i_{b a t}$ and $v_{c_{b}}$, don't contribute to any of the system outputs selected in (4.87); hence, they are unobservable modes. It should be mentioned that, $W_{c}$ and its SVDs depend on the output matrix of the state-space system (Matrix $C$ ); hence, they change based on the output variable selection.

\subsection{Summary}

This chapter focused on developing the dynamic mathematical model of the proposed CSIbased wind turbine system. Detailed models of the aerodynamic conversion, drive train, selfexcited induction generator, Lead Acid battery, and power electronic converters were presented. A reduced-order generic load model including balanced/unbalanced load was developed. By combining the state equations of the system components, the overall model of the system was described by seven inputs, six outputs, and twenty state variables.

To verify the dynamic model, the starting operation of the system was simulated under noload and rated-load scenarios. Then, the behaviour of the system was investigated by simulating its step response. For comparison purposes, simulation results were shown for both switching and average models. The results demonstrated that with respect to the switching model, the average model doesn't represent switching harmonics (as expected), but the dynamics resulting from control system and power system interaction is preserved to a high degree of accuracy.

A linearized model of the system was developed around three operating points. The eigenvalue analysis of the linearized model showed that the open-loop system is locally stable around operating points 1 and 3, but not 2. Gramian method was employed to evaluate the controllability and observability of the system at the three operating points. Based on SVDs, the system is completely controllable at the three operating points, completely observable at operating points 1 and 3 , and not completely observable at operating point 2 , at which the eigenmodes of the battery-side LC filter don't contribute to any of the system outputs selected. 


\section{Chapter 5}

\section{Control System Design for the Proposed PWM-CSI-Based SCIG-WECS}

In Chapter 4, a small-signal linear model for the proposed standalone WECS was developed. The stability analysis showed that the open-loop system was stable around operating points 1 and 3 , but not 2; therefore, the objective of the closed-loop controller design is to stabilize the system and track a class of desired references.

In this chapter:

1) The control objectives of the proposed standalone CSI-based WECS are defined;

2) The structures of the required control loops are designed;

3) The proportional-integral gains of the PI controllers are designed by using a tuning tool available in Matlab/Simulink control toolbox. This tool systematically tunes PI controller for a given plant transfer function or a Simulink model of the plant;

4) The expected performance of the proposed WECS will be verified by simulation;

5) The performance of the proposed WECS under faults will be evaluated; and

5) A dump load-less version of the proposed WECS will be presented.

\subsection{Overview of the control system of the proposed WECS}

The arrangement of the components of the CSI-based WECS, together with the corresponding control blocks, is shown in Fig. 5.1. The system has six control variables, namely, the buck converter duty ratio $d_{b}$, the reduced $\mathrm{H}$-bridge duty ratio $d_{A}$, and the equivalent $d q$-axis modulation indices in the load-side frequency frame $m_{i d}^{+}, m_{i q}^{+}, m_{i d}^{-}$, and $m_{i q}^{-}$. The state variables required to be regulated are the rotor shaft speed $\omega_{r}$ or $\omega_{g}\left(\omega_{r}=P \omega_{g}\right)$, the dc-link current $i_{d c}$, and the $d q-$ axis voltages in the load-side synchronous frame $v_{c i d}^{+}, v_{c i q}^{+}, v_{c i d}^{-}$and $v_{c i q}^{-}$. 


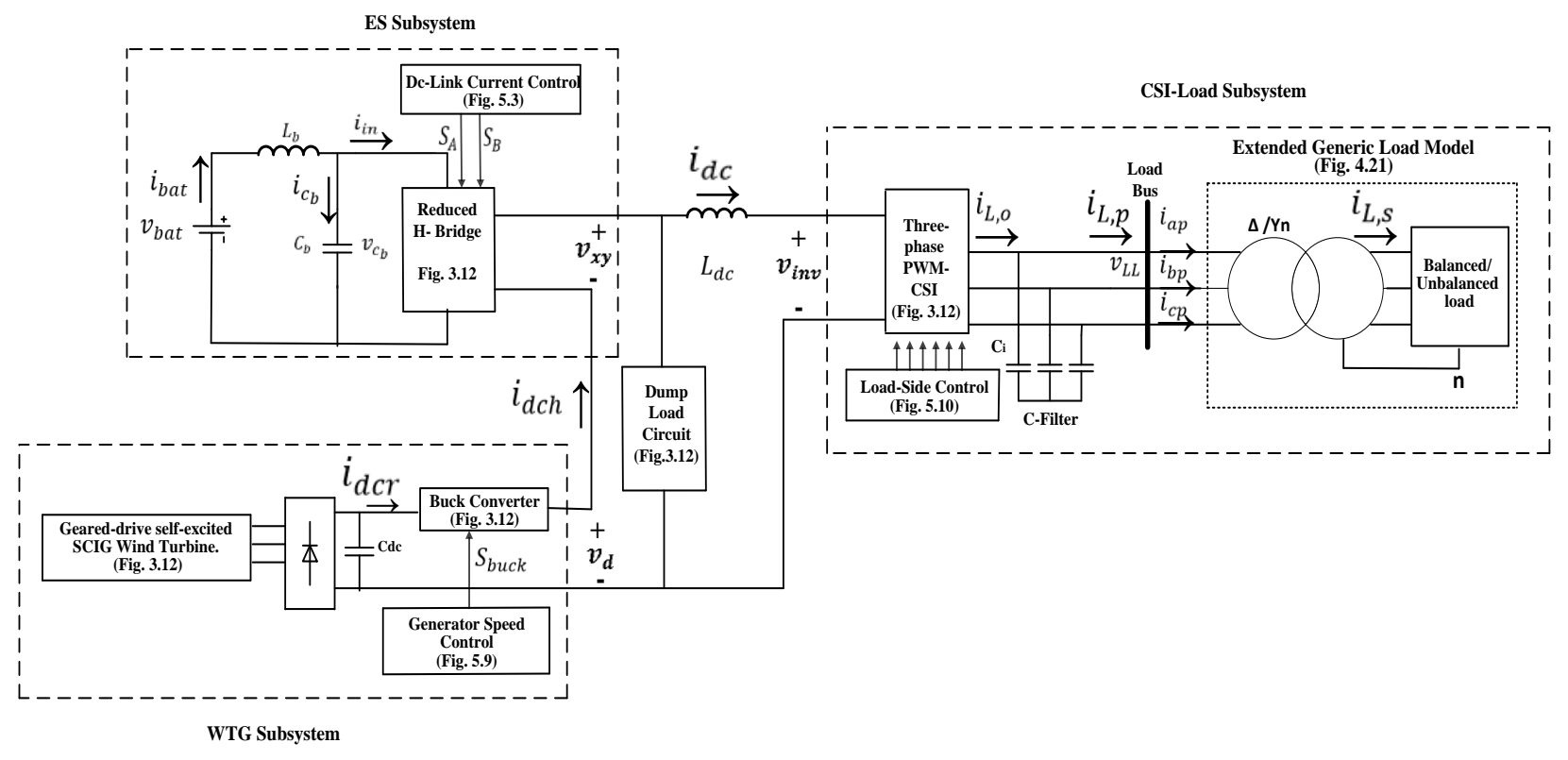

Fig. 5.1: Converters and control blocks in the CSI-based WECS.

The main tasks assigned to the closed loop control system are:

1) DC-link current regulation, resulting in power management among the wind turbine generator, the storage battery bank, the load, and the dump load;

2) Generator speed control to achieve MPPT; and

3) Load-side voltage magnitude and frequency control under both balanced and unbalanced threephase loads.

Since the dc-link inductor provides an energy buffer between the load-side converter (i.e., CSI) and the combination of generator and dc-link current converters (i.e., buck and H-bridge), the loadside converter can be controlled irrespective of dynamic of generator speed and dc-link current. Moreover, due to the mechanical inertia of the turbine, time constant of the generator-speed controller is much longer than the electrical time constant of the dc-link current controller. By proper design of the dc-link current controller, the dc-link current can be regulated at a rate much faster than that of the generator speed. Therefore, dc-link current can be assumed constant when designing the control loop for the generator speed. In other words, a fast (high bandwidth) and stable control of the dc-link current can decouple the controllers of the generator speed, dc-link current, and load-side voltage/frequency; hence, as shown in Fig. 5.1, the system is decomposed 
into three subsystems (i.e., WTG, ES, and CSI-Load), allowing the classical controller design techniques for SISO systems to be applied.

In the following section, a brief description of closed loop control system is given. Then, the structure of the control loops implemented for the proposed CSI- WECS is explained.

\subsection{Closed-Loop Control System}

Fig. 5.2 shows a general closed-loop control system where $x$ is the controlled variable, and $\varepsilon$ the error between the reference value $x^{*}$ and the feedback $x^{\prime}$. The CL controller is composed of three main blocks: controller transfer function $C(s)$, plant transfer function $G(s)$, and feedback transfer function $F(s)$.

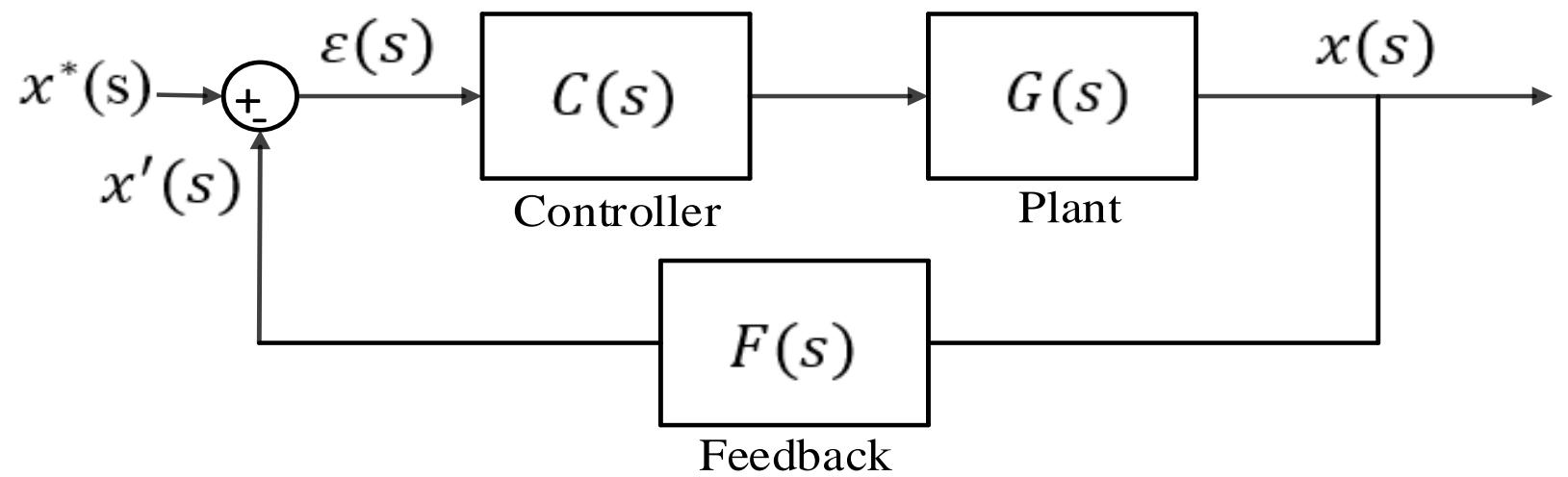

Fig. 5.2: Typical control loop diagram.

In Fig. 5.2, $C(s)$ is the PI controller, defined in s domain by (5.1), where $k_{p}$ and $k_{i}$ are the proportional and integral gains of the controller, respectively.

$$
C(s)=k_{p}+\frac{k_{i}}{s}
$$

$G(s)$ is the plant transfer function of the linearized system.

$F(s)$ is the feedback transfer function. In this work, $F(s)$ is assumed to be unity. However, in an actual setup, it has a gain that is determined by the sensor. In addition, $F(s)$ can represent a low pass filter that might be required to suppress the noise of the measured value.

The open-loop transfer function $(O L T F)$ is defined as 


$$
O L T F=\frac{x^{\prime}(s)}{\varepsilon(s)}=C(s) G(s) F(s)
$$

The closed loop transfer function $(C L T F)$ is

$$
C L T F=\frac{x(s)}{x^{*}(s)}=\frac{C(s) G(s)}{1+C(s) G(s) F(s)}
$$

Setting the denominator of the $C L T F$ equal to zero yields

$$
1+C(s) G(s) F(s)=1+O L T F=0
$$

Equation (5.4) is equivalent to the characteristics equation given in (4.94). In this sense, the open loop transfer function can be used as indicator of stability of the system.

The design of the PI controller parameters can be carried out in time or frequency domain. Matlab/Simulink control toolbox provides PI Tuning tools for such a purpose. In general, the PI parameters are tuned to balance performance (response time) and robustness (stability margins) of the controlled system. It should be mentioned that, in practice, the PI gains are commonly tuned on a trial-and-error basis [146].

\subsection{DC-link Current Control}

This section develops a control algorithm for the dc-link current regulation, followed by the design of the PI-controller parameters.

\subsubsection{DC-link Current Control Scheme}

The operation of CSI requires a regulated current in the dc link which is shared by the generator, storage and load-side converters. DC-link current is controlled through power management between the battery and dump load. The battery is required to absorb the excess power generated from the wind, simply referred to as excess power, during high-wind speed and/or low-load periods, and compensate for the shortage of power during low-wind speed and/or high-load periods or when the wind turbine is not operating. If the battery state-of-charge (SoC) reaches its upper limit, the excess power generated from the wind should be dissipated in the dump load. To achieve this objective, the control scheme shown in Fig. 5.3 is implemented. The reduced H-bridge is controlled by PWM unipolar voltage switching scheme. The control is done by comparing the measured $i_{d c}$ with the dc current reference $\left(i_{d c, r e f}\right)$, processing the error by a PI controller, and producing two control signals, $v_{\text {control }}$ and $-v_{\text {control }}$, that are compared with a triangular carrier 
signal to produce the gating signals for the converter switches. To guarantee a longer operating life for the battery, the battery's SoC is maintained between $25 \%$ and $75 \%$. This is done by multiplying the $v_{\text {control }}$ signal with a control signal $x$ which assumes a value of 0 , when SoC is beyond limits, and a value of 1 , when SoC is within limits.

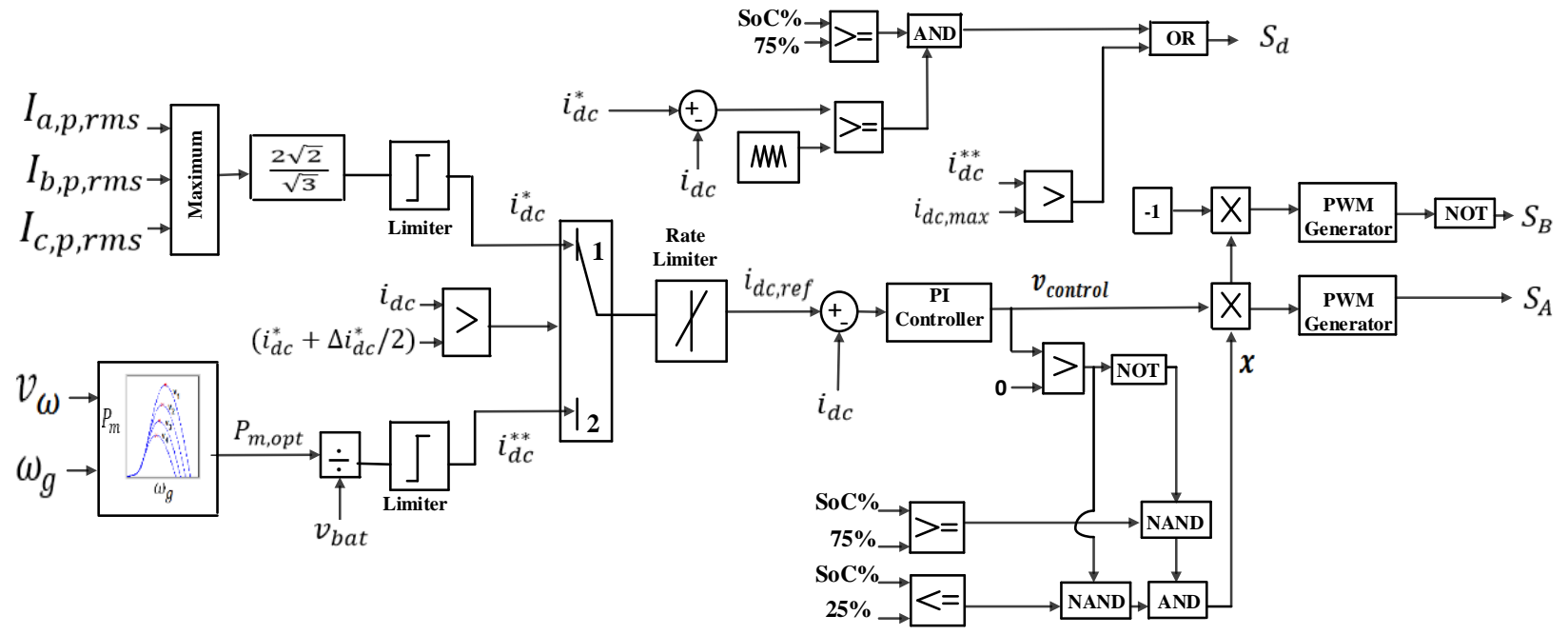

Fig. 5.3: DC-link current control scheme.

The reference of the dc-link current must satisfy the minimum current requirements for both generator- and load-side converters. Considering the load-side converter, it is desired to reduce the dc-link current as the power demand is reduced, in order to reduce $i_{d c}^{2} R_{d c}$ and CSI losses, and avoid CSI's low modulation indices which increase harmonic distortion in the output voltage. As a result, the dc-link current reference, $i_{d c}^{*}$, is determined as follows:

1) On a per-phase basis, the CSI's ac-side line current $\left(i_{L, o}\right)$ is equal to the sum of the filter capacitor current and the primary-side line current $\left(i_{L, p}\right)$ of the $\Delta / Y n$ transformer, which is related to the secondary-side line current $\left(i_{L, S}\right)$ through the $\Delta / Y$ transformation ratio $N$.

2) The fundamental component of the current in the C-filter is considerably smaller than that of the transformer primary-side; hence, it can be neglected. Moreover, the high-order harmonic components of the transformer primary-side current can be neglected as they are significantly reduced by the $\mathrm{C}$-filter. Hence, the rms value of transformer primary-side current, $I_{L, p, r m s}$, can be 
assumed to be equal to the rms value of the fundamental component of the ac-side line current, $I_{L, o, 1, r m s}$, i.e.,

$$
I_{L, p, r m s}=I_{L, o, 1, r m s}
$$

From (1.3), when $m_{i}=1$,

$$
I_{L, o, 1, r m s}=\frac{\sqrt{3}}{2 \sqrt{2}}\left(i_{d c}\right)
$$

Based on (5.5) and (5.6), and considering the turns-ratio $N$ of the $\Delta / Y n$ transformer, $i_{d c}^{*}$ can be obtained for the case of balanced load as

$$
i_{d c}^{*}=\frac{2 \sqrt{2}}{\sqrt{3}} I_{L, p, r m s}=\frac{2 \sqrt{2}}{N} I_{L, s, r m s}
$$

Equation (5.7) states that $i_{d c}^{*}$ can be set to different values based on either measured primary-side current $\left(I_{L, p, r m s}\right)$ or secondary-side current $\left(I_{L, s, r m s}\right)$. To account for unbalanced load, $i_{d c}^{*}$ is set based on the highest $I_{L, p, r m s}$ of the three phases so that a high-enough $i_{d c}$ that suits all phases is guaranteed. The modulation index corresponding to the phase with the largest rms current is 1 , whilst it is less than 1 for the other two phases. Even though it is known that as the load current decreases $i_{d c}$ can be decreased, there should be a minimum $i_{d c}$ based on which the dc-link inductor is designed (see equation (3.12)). In this work, the minimum demand is assumed to be $20 \%$ of the rated load; hence, only $20 \%$ of the rated dc-link current will be enough to satisfy the load. However, in order to reduce the size of the bulky dc-link inductor, it is desired to increase the minimum limit for the dc-link current, which, nevertheless, implies higher dc-link and inverter losses. As a trade-off between dc-link inductor size reduction and dc-link efficiency improvement, the minimum reference for $i_{d c}$ is set at $40 \%$ of the rated $i_{d c}$ in order to avoid modulation indices below 0.5 . This current is referred to as $i_{d c, \min }$. On the other hand, there should be an upper limit for $i_{d c}$ to avoid exceeding the current limit of power IGBTs. This current is referred to as $i_{d c, \max }$. Load $k V A$ should be managed so that the required dc-link current will never exceed this upper limit. $i_{d c, \max }$ has to be lower than the battery current limit.

As noticed from Fig. 5.1, the reduced H-bridge terminals are connected in series with the dc link inductor during non-dumping periods (i.e., $i_{d c h}=i_{d c}$ ). Thus, the battery-side current $\left(i_{\text {in }}\right)$ during charging or discharging is composed of pulses of magnitude $i_{d c}$ or $-i_{d c}$, separated by zero-current periods. Therefore, the battery average power $\left(P_{\text {bat,avg. }}\right)$ can be calculated using $(5.8)$, where $v_{\text {bat }}$ is the battery terminal voltage, and $i_{b a t, a v g}$. and $v_{x y, a v g}$. the average values of the filtered battery 
current and reduced-H-bridge output voltage, respectively, with a conversion efficiency of $100 \%$ assumed.

$$
P_{\text {bat,avg. }}=\left(v_{\text {bat }}\right)\left(i_{\text {bat,avg. }}\right)=\left(v_{x y, a v g .}\right)\left(i_{d c}\right)
$$

At high wind speeds and low- or no-load conditions, the excess power to be absorbed by the battery is high, requiring a high $i_{d c}$. However, $i_{d c}^{*}$ is set by (5.7) according to load demand. Due to the fact that the maximum of $v_{x y, a v g}$. does not exceed $v_{b a t}$, the maximum average power that the battery is allowed to absorb under $i_{d c}^{*}$ is $v_{b a t} i_{d c}^{*}$. This particular power is referred to as $P_{b a t}^{*}$. If the excess power exceeds $P_{b a t}^{*}$, the dc-link current will be forced to go from $i_{d c}^{*}$, which is set low to reduce inverter and dc-link losses, to a higher value required by the battery to absorb whatever excess power is available. This implies a conflict between improving inverter and dclink efficiencies and MPPT. Because wind power is intermittent, and hence may not be available when it is needed most, this work gives the priority to achieving maximum power and storing all excess power as long as the battery bank has free capacity and its current rating allows. However, the advantage of reducing $i_{d c}$ at low load should be preserved as long as it doesn't contradict MPPT. In other words, the reference of $i_{d c}$ is initially set at $i_{d c}^{*}$ required by the load. This will allow the battery to absorb excess power below or equal to $P_{b a t}^{*}$. If excess power goes beyond $P_{b a t}^{*}$, an $i_{d c}$ higher than $i_{d c}^{*}$ is required. In this case, the reference of $i_{d c}$ is set as required by the battery to absorb the entire excess wind power, as long as the battery current limit and SoC upper limit are not exceeded. This current is denoted as $i_{d c}^{* *}$. Assuming a lossless system, equation (5.9) can be used to find $i_{d c}^{* *}$, where $P_{m, o p t}$ is the steady-state optimum wind power, obtained from wind power-shaft speed look-up table based on Fig. 4.3, and $P_{L}$ is the load power. It should be noted that excess power will not go beyond $P_{b a t}^{*}$ unless $P_{m, o p t} \gg P_{L}$; then, $P_{m, o p t}-P_{L} \approx P_{m, o p t}$.

$$
i_{d c}^{* *}=\frac{P_{m, o p t}-P_{L}}{v_{b a t}} \approx \frac{P_{m, o p t}}{v_{b a t}}
$$

The mechanism of determining the dc-link current reference $\left(i_{d c, r e f}\right)$ is incorporated in the dclink current control scheme of Fig. 5.3 by employing an automatic software switch, which by default assumes position 1, corresponding to $i_{d c}^{*}$. If $i_{d c}$ exceeds $i_{d c}^{*}$ plus permitted ripple, the switch assumes position 2, corresponding to $i_{d c}^{* *}$. A limiter is used to ensure the dc-link current reference always remains between $i_{d c \text {, min }}$ and $i_{d c \text {, max }}$. As noticed from Fig. 5.3, $i_{d c}^{* *}$ is prevented from exceeding $i_{d c, \max }$ by engaging the dump load, thus limiting the power absorbed by the battery to 
$i_{d c, \text { max }} v_{b a t}$. Moreover, a falling/rising-rate limiter block is used in order to change $i_{d c, r e f}$ gradually to the desired value; this is because the dc choke will not allow fast changes in $i_{d c}$.

\subsubsection{Parameters Design of DC-link Current PI Controller}

As described in subsection 5.3.1, the dc-link current is set based on the load demand (i.e., $i_{d c, r e f}=i_{d c}^{*}$ ) or the optimal wind power (i.e., $i_{d c, r e f}=i_{d c}^{* *}$ ). In other words, the response time of the dc-link current controller should be fast enough to respond to load variation as well as wind speed variation. For aggregated residential load, combining a group of houses, a considerable change in demand can occur in the range of minutes. On the other hand, wind speed may vary very dramatically in a matter of few seconds [147]. Therefore, the bandwidth of the dc-link current controller should be higher than the frequency of wind speed variation. In general, the higher the bandwidth, the better the performance of the controller. In practice, however, rejection error of high-frequency disturbances and the ripple threshold for control signal set limits on the controller bandwidth [146].

In order to show the system performance under various wind and load conditions during short intervals of time, the dc-link control bandwidth is selected in range of tens of hertz, enabling very short rise time as well as response time. The parameters of PI controller of dc-link current controller are designed to satisfy the following time-domain specifications:

- The step response settling time of less than 0.05 second;

- The step response steady-state error of zero; and

- The overshoot/undershoot of less than $10 \%$.

The frequency-domain specifications are as follows:

- Controller Bandwidth $\geq 40 \mathrm{~Hz}$ (250 rad/s); and

- Stability Phase Margin $\geq 60^{\circ}$.

Based on transfer functions (provided in Table 5.1) derived from the small-signal model of ES subsystem (given in Appendix E), Matlab/Simulink PI tuning tools are used to tune the PI parameters of the dc-link controller at the three operating points of linearization described in chapter 4 (Table 4.7). The Tuning function can also be achieved based on the Simulink model of the ES subsystem. 
Table 5.1: Small-signal transfer functions of the ES-subsystem.

\begin{tabular}{cl}
\hline Operating point & Transfer Function $\left(\boldsymbol{G}_{\boldsymbol{i}_{\boldsymbol{d} \boldsymbol{c}}}=\frac{\boldsymbol{i}_{\boldsymbol{d c}}}{\mathbf{2 d}_{\boldsymbol{A}} \mathbf{- 1}}\right)$ \\
\hline o.p.1 & $\frac{2.55 \times 10^{4} s^{2}+3.562 \times 10^{8} s+1.051 \times 10^{12}}{s^{3}+1.432 \times 10^{4} s^{2}+4.626 \times 10^{7} s+1.567 \times 10^{10}}$ \\
o.p.2 & $\frac{9.752 \times 10^{4} s^{2}+1.36 \times 10^{9} s+3.994 \times 10^{12}}{s^{3}+1.394 \times 10^{4} s^{2}+4.095 \times 10^{7} s}$ \\
o.p.3 & $\frac{2.523 \times 10^{4} s^{2}+3.516 \times 10^{8} s+1.031 \times 10^{12}}{s^{3}+1.362 \times 10^{4} s^{2}+3.64 \times 10^{7} s-1.335 \times 10^{10}}$ \\
\hline
\end{tabular}

Fig. 5.4 shows the tuning results of the dc-link current $\left(i_{d c}\right)$ controller at o.p.1. The tuning time/frequency domain results and step responses of the closed loop $i_{d c}$ controller show that the system meets the design conditions specified above. Same procedures are carried out at o.p.2 and o.p.3, with the tuning results given in Fig. 5.5 and Fig. 5.6, respectively. The resulting $k_{p}$ and $k_{i}$ are displayed in Table 5.2.

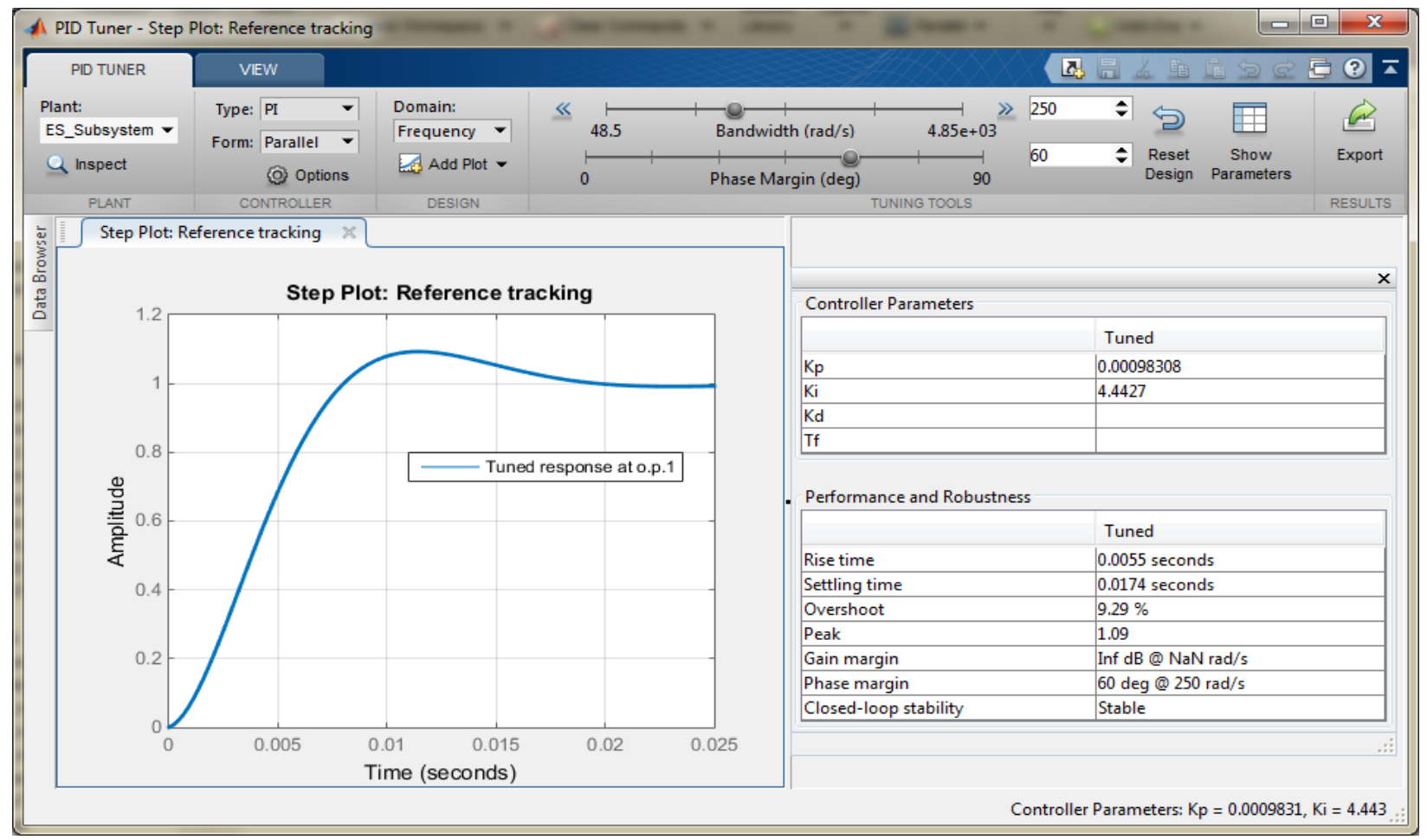

Fig. 5.4: PI tuning of the dc-link current controller at o.p.1. 


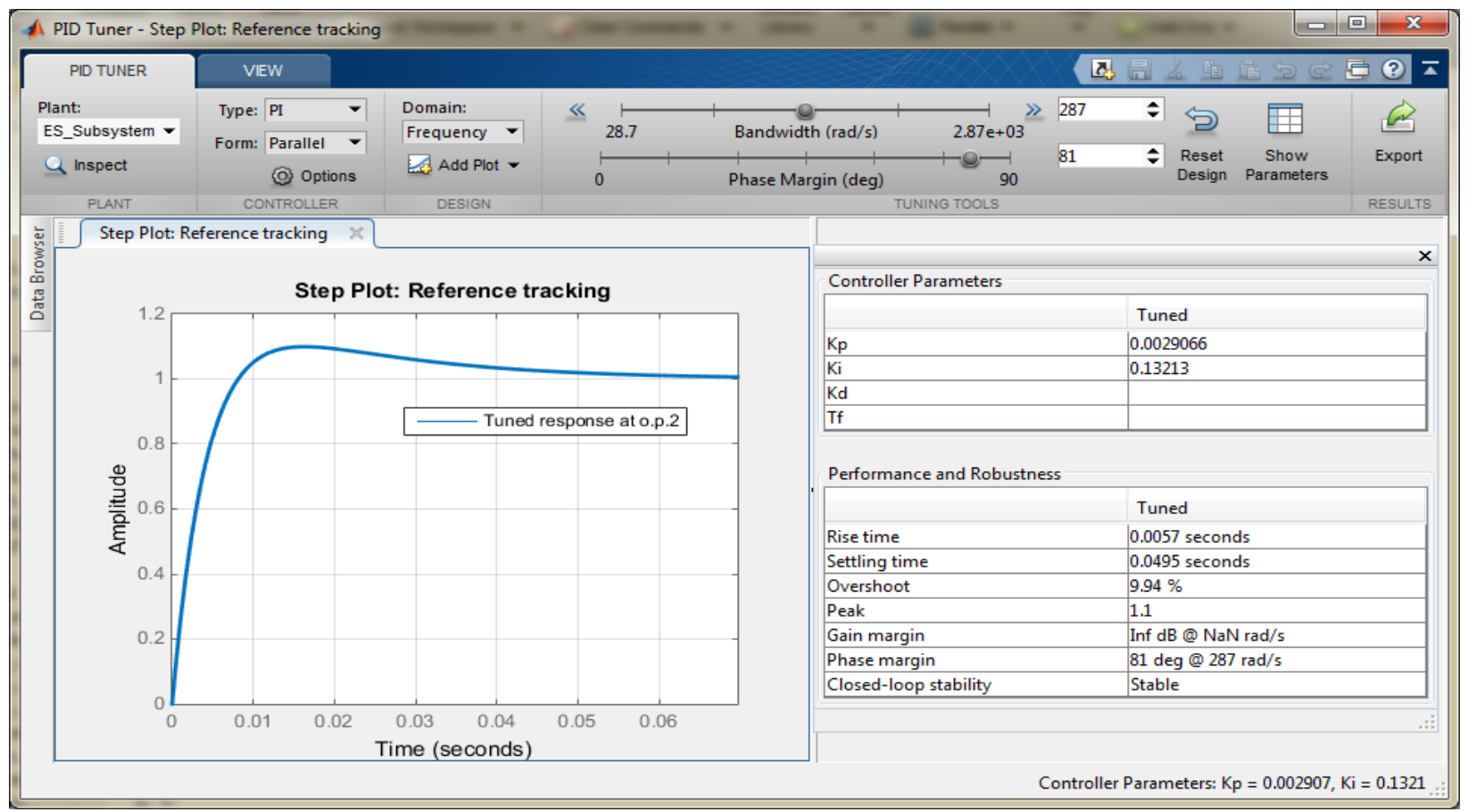

Fig. 5.5: PI tuning of the dc-link current controller at o.p.2.

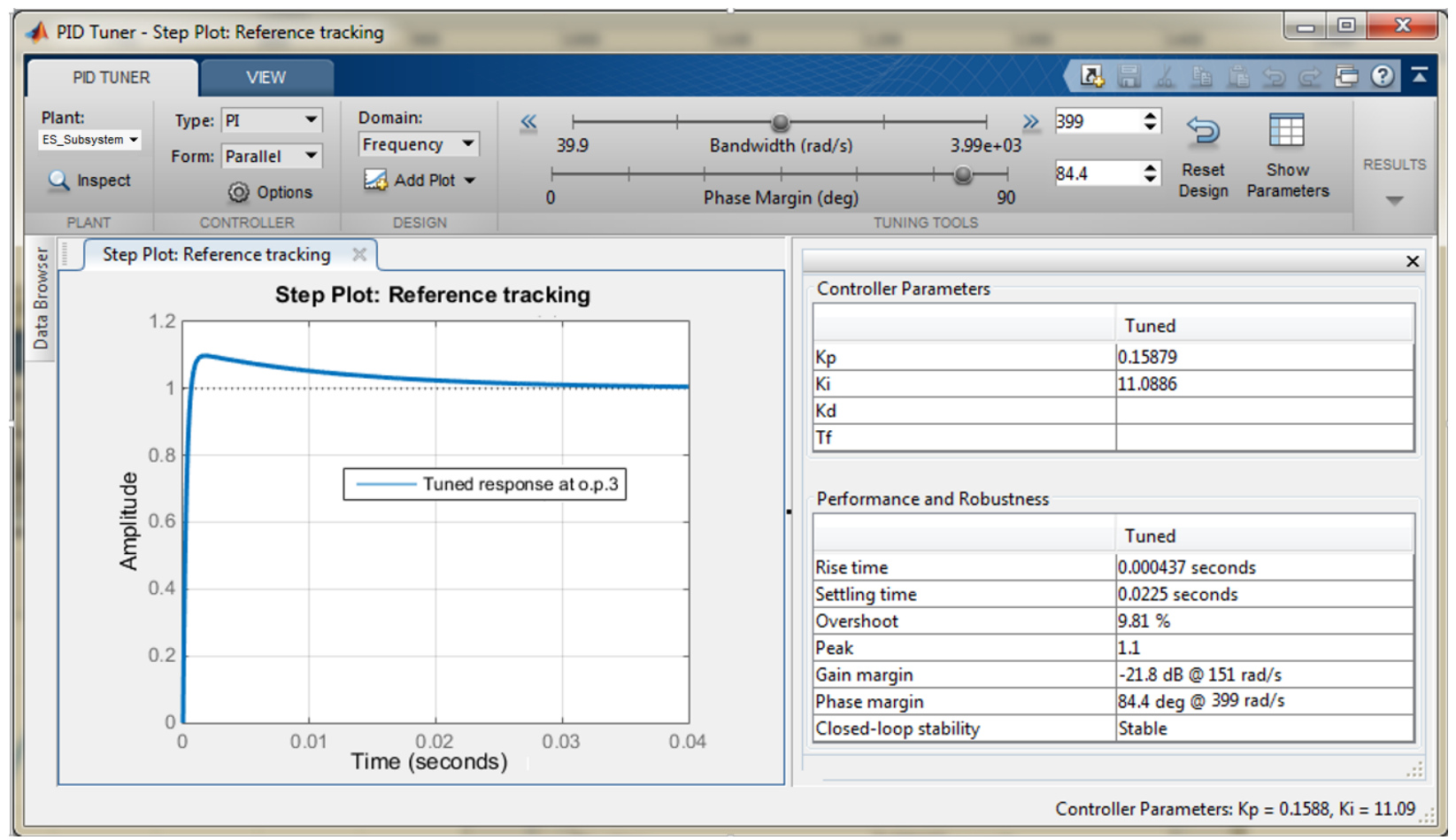

Fig. 5.6: PI tuning of the dc-link current controller at o.p.3. 
Based on the small-signal transfer functions and PI parameters given in Table 5.1 and Table 5.2, respectively, Fig. 5.7 shows the small-signal step responses of dc-link current from one operating point to a new steady-state value. In Fig. 5.7(a), the system was operating at o.p.1 ( $i_{d c, o . p .1}=$ $51 \mathrm{~A}$ ) before a $25 \%$ step increase is applied at $\mathrm{t}=0.5 \mathrm{~s}$. The new steady-state value of $i_{d c}$ is reached in about $0.02 \mathrm{~s}$, with an overshoot of about 9.3\%. These values match the time-domain characteristics obtained from the PI tuning tools (see Fig. 5.4). Because the dc choke will not allow step change in $i_{d c}$, the reference $i_{d c}$ is applied as ramp signal, as in Fig. 5.7(b), resulting in slower response time and less overshoot. Similar observations are noticed from Fig. 5.7(c) - (f) where step change and ramp signal are applied to $i_{d c}$ at o.p.2 and o.p.3.

(a) Step response of dc-link current under PI gains at o.p.1

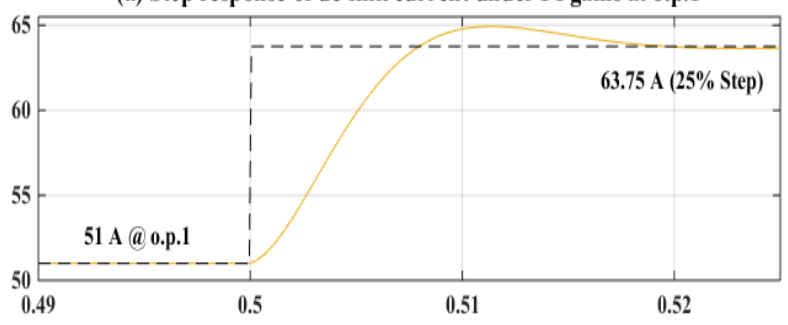

(c) Step response of dc-link current under PI gains at 0.p.2

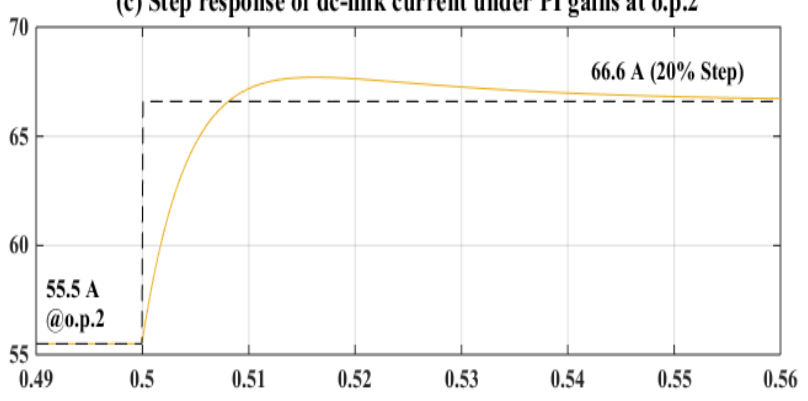

(e) Step response of dc-link current under PI gains at 0.p.3

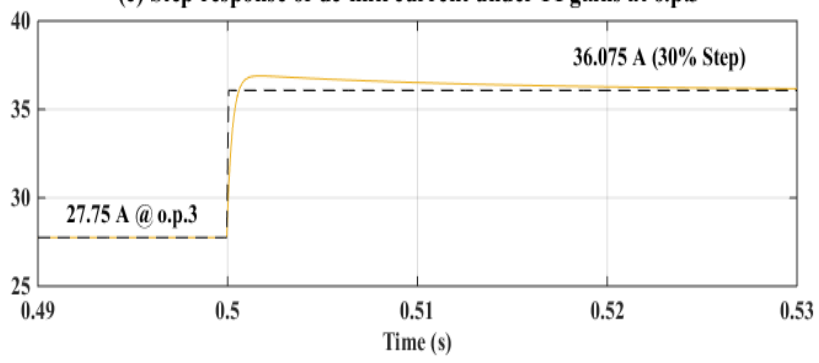

(b) Ramp response of dc-link current under PI gains at 0.p.1

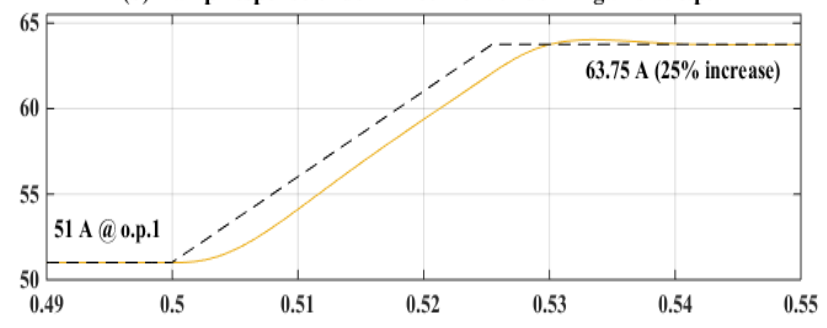

(d) Ramp response of dc-link current under PI gains at 0.p.2

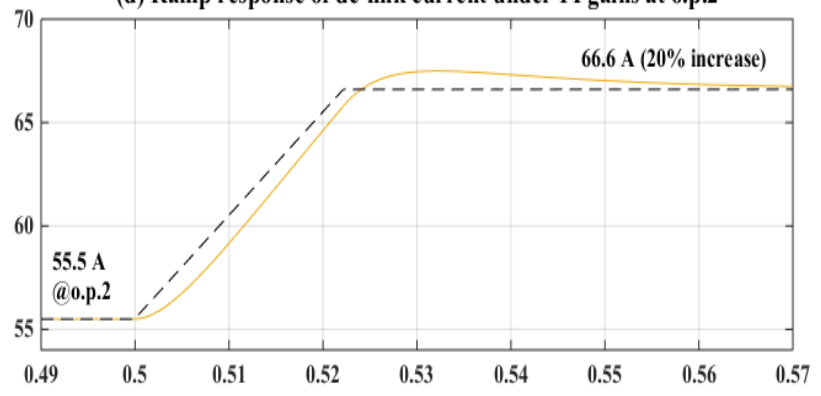

(f) Ramp response of dc-link current under PI gains at 0.p.3

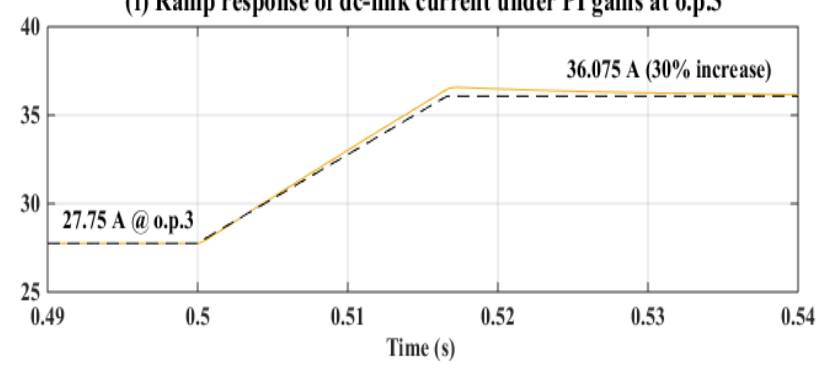

Fig. 5.7: Step/Ramp response of dc-link current at the three operating points. 


\subsection{Generator Speed MPPT Control}

This section describes control algorithm for the generator speed followed by the design of the PI-controller parameters.

\subsubsection{MPPT Control Scheme}

In a variable-speed wind turbine, the generator is controlled to extract the maximum power available from the wind. Different techniques for maximum power point tracking, reported in the literature, have been reviewed in [148]. They can be categorized under perturbation and observation $(\mathrm{P} \& \mathrm{O})$ based techniques and Look-up table based techniques. $\mathrm{P} \& \mathrm{O}$ techniques are simple and require no prior knowledge of the system parameter. However, due to its slow operation, $\mathrm{P} \& \mathrm{O}$ technique may not be efficient for wind turbines, where the dynamics of wind is very fast (i.e., wind speed changes quite fast in a matter of seconds) [147]. On the other hand, the look-up table-based techniques, such as power or torque-signal feedback, and tip-speed ratio (TSR) techniques, are commonly used in wind turbines although they require speed sensors and a pre-programmed look-up table of the turbine data. Among the look-up table techniques, TSR technique can provide the fastest control action because it depends on direct measurement of the wind speed and sets the control reference instantaneously, resulting in more energy production [147],[149]. In this chapter, therefore, the TSR technique is implemented to achieve MPPT.

Fig. 5.8 shows a typical power-wind speed curve for a variable-speed wind turbine. The curve is divided into three regions. From cut-in to rated wind speed (i.e., region 1), the turbine is controlled at the maximum performance coefficient $\left(C_{p_{\max }}\right)$ corresponding to the optimum tip speed ratio $\left(\lambda_{\text {opt }}\right)$; thus, maximum power available from wind is extracted. At wind speeds higher than the rated value (i.e., region 2), the turbine is aerodynamically controlled in order to limit the extracted power to the rated value. Such a control is carried out either by stall or pitch regulation mechanism. For a fixed-pitch wind turbine, the blades are aerodynamically designed to achieve passive stall. Moreover, the rotor speed of a fixed-pitch turbine can be adjusted by furling control or electronic brakes. In order to protect the turbine in region 3, it must be stopped at cut-out speed. Furling control can significantly reduce the rotor speed, but it may not be able to stop it. Therefore, a mechanical, electronic or hydraulic brake is used to bring the rotor to rest after its speed is reduced 
by furling. The brake can also be used to ramp off the generated wind power in emergency cases such as sudden disconnection of load while the storage unit is not capable of absorbing the generated power. Even in the presence of brake, a dump load is necessary to complete the protection scheme, especially during transients.

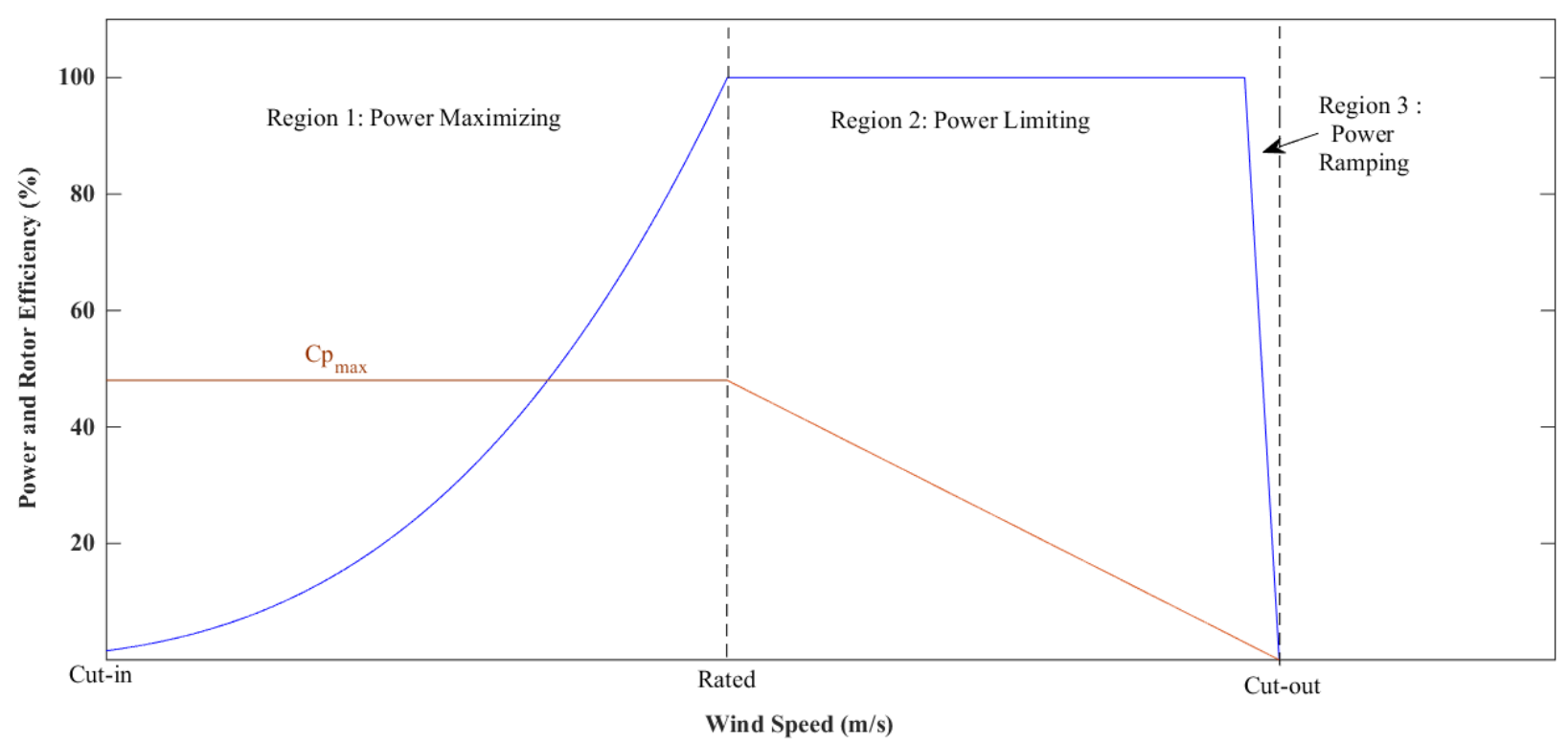

Fig. 5.8: Typical turbine power versus wind speed curve.

In the proposed WECS, shown in Fig. 5.1, the main function of the generator-side converter (i.e., buck converter) lies in region 1. The converter, as shown in Fig. 5.9, is controlled to extract maximum power from wind by regulating the generator shaft speed at the optimum value corresponding to the present value of wind speed. The same concept has been applied in [73],[74] to control a direct-drive PMSG in a grid-connected WECS. The control is based on keeping the tip speed ratio at the optimal value. As revealed from equation (4.4), at a fixed tip speed ratio, the turbine's rotational speed is linearly related to the wind speed (i.e., $\omega_{m} \propto v_{W}$ ). Therefore, as shown in Fig. 5.9, the reference signal for generator angular speed $\left(\omega_{g, o p t}\right)$ is produced based on the optimal tip speed ratio $\left(\lambda_{\text {opt }}\right)$, the measured wind speed $\left(v_{w}\right)$, gearbox ratio $\left(n_{\text {gear }}\right)$ to account for the speed level conversion, and the turbines radius $(r)$. The error between $\omega_{g, o p t}$ and the measured speed $\omega_{g}$ is amplified by a PI controller, leading to the generation of gating pulses for the buck switch, $S_{b u c k}$, through PWM process. As a result, the rectifier output current $\left(i_{d c r}\right)$, the SCIG stator 
current $\left(i_{d s}\right)$, the SCIG counter torque $\left(T_{e}\right)$ and finally the shaft speed $\left(\omega_{g}\right)$ are adjusted to achieve maximum power.

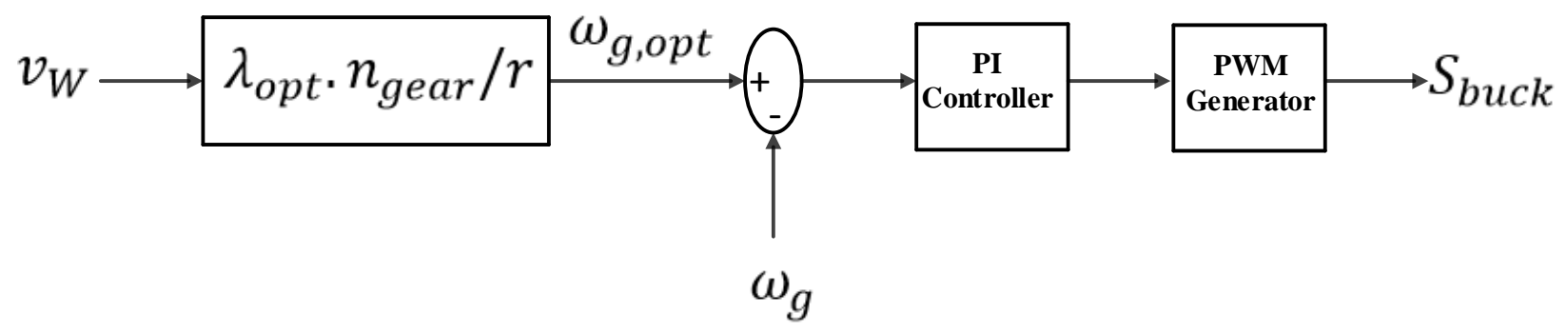

Fig. 5.9: MPPT Controller.

\subsubsection{Parameter Design of Generator Speed Control Loop}

As mentioned in section 5.1, the generator speed $\left(\omega_{g}\right)$ control loop is designed to be much slower than that of the dc-link current $\left(i_{d c}\right)$ control loop. In view of this, the design of $\omega_{g}$ controller does not take into account the dc-link dynamics based on the assumption that $i_{d c}$ is quickly and robustly regulated by the ES-subsystem. Accordingly, the PI parameters of generator speed controller are designed to satisfy the following time-domain specifications:

- The step response settling time of less than 0.5 second;

- The step-response steady-state error of zero; and

- The overshoot/undershoot of less than $10 \%$.

The frequency-domain specifications are as follows:

- Controller Bandwidth $\geq 4 \mathrm{~Hz}$ (25 rad/s) (One-tenth of that in dc-link current control loop); and

- Stability Phase Margin $\geq 60^{\circ}$.

By applying a procedure similar to that for dc-link current controller design, PI parameters of the generator-speed controller are tuned by Matlab/Simulink tuning tools based on the Simulink model of the linearized WTG subsystem. The resulting $k_{p}$ and $k_{i}$ are displayed in Table 5.2. 


\subsection{Load-Side Control}

This section describes the control algorithm for the load-side voltage/frequency, followed by the design of the PI-controller parameters.

\subsubsection{Load-Side Control Scheme}

The unbalanced load currents lead to unbalanced line voltages at the load bus. Voltage imbalance can cause serious problems to three-phase loads, especially motor loads. Therefore, it is compulsory to compensate for the voltage imbalance at the load bus so that the voltage unbalance factor (VUF), defined as the ratio of fundamental component of negative-seq voltage to that of positive-seq voltage, does not exceed the permissible limit of $1 \%$.

In the proposed WECS, shown in Fig. 5.1, the $\Delta / Y_{n}$ transformer isolates the zero-seq component of load current. Therefore, as shown in Fig. 5.10, the unbalanced voltage across the C-filter $\left(v_{c_{\text {iabc }}}\right)$ is decomposed into symmetrical positive-seq $\left(v_{c_{i a b c}}^{+}\right)$and negative-seq $\left(v_{c_{i a b c}}^{-}\right)$components based on transformation matrices given in (5.10) and (5.11), respectively.

$$
\begin{aligned}
& {\left[\begin{array}{l}
v_{c_{i a}}^{+} \\
v_{c_{i b}}^{+} \\
v_{c_{i c}}^{+}
\end{array}\right]=\frac{1}{3}\left[\begin{array}{ccc}
1 & a & a^{2} \\
a^{2} & 1 & a \\
a & a^{2} & 1
\end{array}\right]\left[\begin{array}{l}
v_{c_{i a}} \\
v_{c_{i b}} \\
v_{c_{i c}}
\end{array}\right]} \\
& {\left[\begin{array}{l}
v_{c_{i}}^{-} \\
v_{c_{i b}}^{-a} \\
v_{c_{i c}}^{-}
\end{array}\right]=\frac{1}{3}\left[\begin{array}{ccc}
1 & a^{2} & a \\
a & 1 & a^{2} \\
a^{2} & a & 1
\end{array}\right]\left[\begin{array}{l}
v_{c_{i a}} \\
v_{c_{i b}} \\
v_{c_{i c}}
\end{array}\right]}
\end{aligned}
$$

where $a=e^{j 2 \pi / 3}$.

The concept of sequence decomposition has been used in [150],[151] to control a VSI supplying an unbalanced load. The big advantage of sequence decomposition is that it allows decoupled control of the positive and negative sequence voltage components. However, it delays the measured voltage by one-fourth of the period at the fundamental frequency [151]. In Fig. 5.10, the ability of synchronous $d q$ control to achieve zero steady-state error is utilized for each symmetrical component. The positive-seq $d$-axis voltage $\left(v_{c i d}^{+}\right)$is compared with the desired value of $1 \mathrm{pu}$ and the error is processed in a PI controller. All other voltages (i.e., $v_{c i q}^{+}, v_{c i d}^{-}, v_{c i q}^{-}$) are kept at zero value. This guarantees achieving a positive seq balanced voltage. The $d q$-frame output signals of the PI controllers are transformed into abc frame and the resulting signals of the same 
phases are added together to produce the modulating signals $\left(m_{i_{a b c}}\right)$ to be applied to the PWM generator to generate gating pulses for the CSI switches. The frequency is set at the desired value (i.e., 50 or $60 \mathrm{~Hz}$ ) in open-loop control.

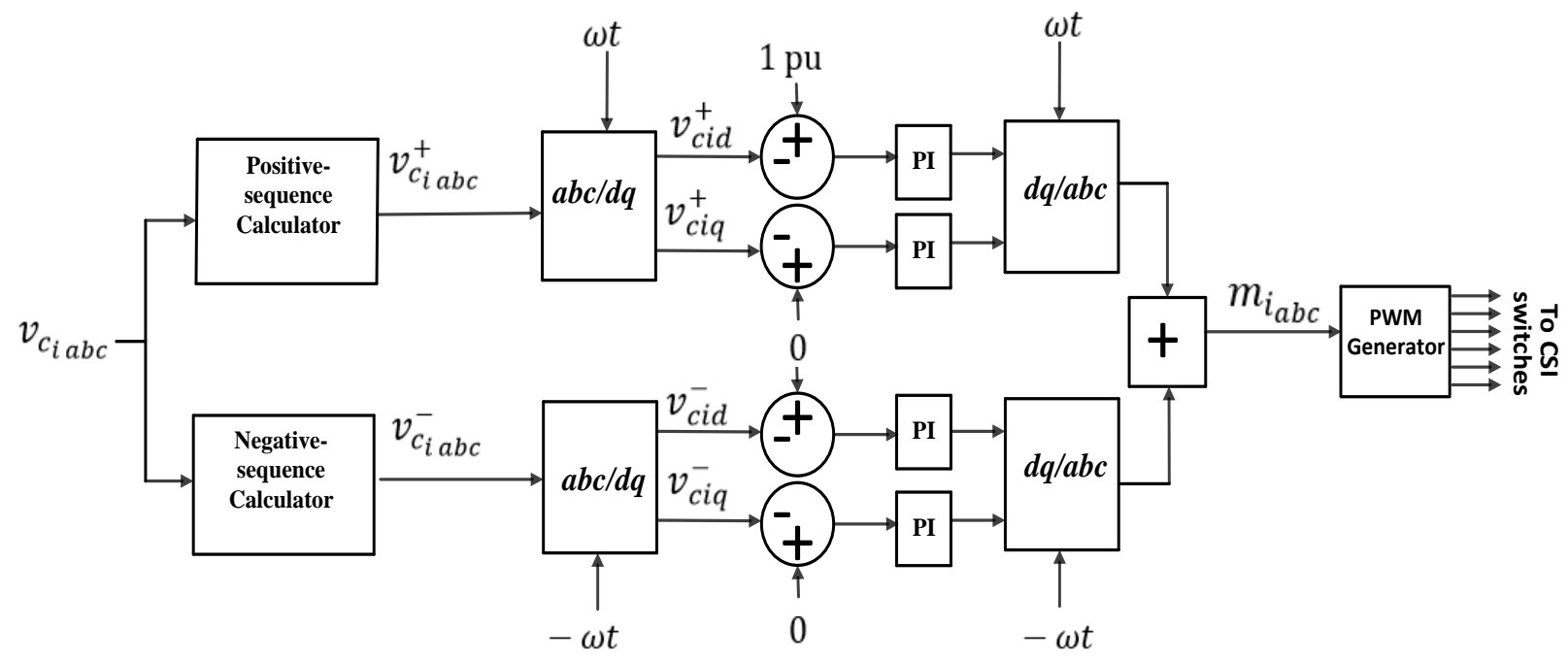

Fig. 5.10: Load-side synchronous $d q$ frame control scheme.

\subsubsection{Parameter Design of Load-Side Control Loop}

As mentioned in section 5.1, the dc-link inductor provides an energy buffer between CSI-Load subsystem and the combination of WTG and ES subsystems. In addition, with very fast dc-link current $\left(i_{d c}\right)$ control loop, $i_{d c}$ is considered as constant input to the CSI-Load subsystem. As a result, the load-side voltage and frequency can be controlled irrespective of wind speed variation. The parameters of PI controller of load-side controller are designed to satisfy the following timedomain specifications:

- The step response settling time of less than 0.1 second;

- The step-response steady-state error of zero; and

- The overshoot/undershoot of less than $10 \%$.

The frequency-domain specifications are as follows:

- Controller Bandwidth $\geq 20 \mathrm{~Hz}$ (125 rad/s) (Half of that in dc-link current loop); and

- Stability Phase Margin $\geq 60^{\circ}$. 
Employing the same Matlab/Simulink tool that was used for tuning dc-link current and generator-speed controllers, $k_{p}$ and $k_{i}$ of the load-side controllers are obtained as given in Table 5.2. In the table, $k_{p}^{+}$and $k_{i}^{+}$are the gains of PI controllers of positive sequence $d q$ voltages and $k_{p}^{-}$and $k_{i}^{-}$the gains of PI controllers of negative sequence $d q$ voltages.

Table 5.2: PI gains of the closed loop system controllers at the three operating points.

\begin{tabular}{|c|c|c|c|c|c|c|}
\hline & \multicolumn{2}{|c|}{$\begin{array}{l}\text { Generator speed } \\
\text { controller }\end{array}$} & \multicolumn{2}{|c|}{$\begin{array}{l}\text { DC-link current } \\
\text { controller }\end{array}$} & \multicolumn{2}{|c|}{ Load-Side Controllers } \\
\hline & \multirow[b]{2}{*}{$k_{p}$} & \multirow[b]{2}{*}{$k_{i}$} & \multirow[b]{2}{*}{$k_{p}$} & \multirow[b]{2}{*}{$k_{i}$} & $k_{p}^{+}$ & $k_{i}^{+}$ \\
\hline & & & & & $k_{p}^{-}$ & $k_{i}^{-}$ \\
\hline \multirow[b]{2}{*}{ o.p.1 } & \multirow[b]{2}{*}{42.7024} & \multirow[b]{2}{*}{284.0791} & \multirow[b]{2}{*}{0.00098308} & \multirow[b]{2}{*}{4.4427} & 0.24761 & 5.6081 \\
\hline & & & & & 0.01211 & 5.5194 \\
\hline \multirow[b]{2}{*}{ o.p. 2} & \multirow[b]{2}{*}{39.4928} & \multirow[b]{2}{*}{268.823} & \multirow[b]{2}{*}{0.0029066} & \multirow[b]{2}{*}{0.13213} & 0.22753 & 5.1534 \\
\hline & & & & & 0.04352 & 5.0621 \\
\hline \multirow[b]{2}{*}{ o.p.3 } & \multirow[b]{2}{*}{9.3191} & \multirow[b]{2}{*}{119.4989} & \multirow[b]{2}{*}{0.15879} & \multirow[b]{2}{*}{11.0886} & 0.45507 & 10.3068 \\
\hline & & & & & 0.03541 & 9.8675 \\
\hline
\end{tabular}

\subsection{Simulation Verification}

In this section, the closed-loop control systems for the proposed SCIG-CSI-WECS are simulated in Matlab/Simulink environment for a 20kW standalone WECS using a 460Vrms SCIG and the extended generic load model requiring a regulated voltage of $380 \mathrm{~V} / 220 \mathrm{Vrms}$ at $60 \mathrm{~Hz}$. The system's parameters are given in Appendix A (Table A.2). First, the system performance is examined under variable turbulence-free wind speed and well-damped three-phase load conditions. Next, the effect of system inertia and frequency of wind speed variation on MPPT is studied. Finally, the performance of the synchronous $d q$ controller under load conditions with various dynamics and steady-state characteristics will be evaluated.

In the following simulations, the closed-loop PI controllers are tuned based on gain-scheduling given in Table 5.2 for the three operating points selected for linearization. However, in order to illustrate the performance of the control schemes under various conditions, the system is also simulated at other operating points. At each operating point, Matlab/Simulink tuning tools is used to linearize the system Simulink model, tune the controller gains, and reconcile the gain values to provide smooth transition between operating conditions. 
Unless otherwise specified, the simulation results of this section are obtained based on switching model of the entire CSI-based WECS.

In general, loads in remote areas are composed of $80 \%$ constant-impedance and $20 \%$ constantpower components [152]. Since the load is dominantly of constant-impedance type, the simulation results presented in this section are based on the assumption of $100 \%$ constant-impedance loads.

\subsubsection{System Performance under Various Wind Speed and Load Conditions}

The performance of the system is examined under variable wind speed and balanced/unbalanced three-phase load as follows:

\section{A) Variable Balanced Load}

The system is operated under variable wind speed and balanced three-phase load, according to the wind speed and load profiles given in Table 5.3. The wind speed is considered a constant signal at its average value in each sub-period. The rated load is $6.67 \mathrm{~kW}$ and $3.33 \mathrm{kVar}$ per phase (i.e., three-phase powers are $P^{+}=20 \mathrm{~kW}, Q^{+}=10 \mathrm{kVar}$, and $\left.Q_{p d}^{-}=Q_{p q}^{-}=0\right)$. Throughout the simulation period, the load profile has the same dynamic characteristics $\left(d=100 \mathrm{~s}^{-1}\right.$ and $\omega_{o}=$ $75 \mathrm{rad} / \mathrm{s}$ ). The initial SoC of the battery is 50\%. Simulation results are shown in Fig. 5.11.

Table 5.3: Wind speed and load conditions for simulation results of Fig. 5.11 and Fig. 5.14.

\begin{tabular}{cccc}
\hline Time $(\mathbf{s})$ & Wind speed $(\mathbf{m} / \mathbf{s})$ & \% of rated load & DC-link current reference $\mathbf{( A )}$ \\
\hline 0 & 12 & 0 & $i_{d c}^{* *}=51$ \\
0.5 & 12 & 20 & $i_{d c}^{* *}=51$ \\
1.0 & 12 & 100 & $i_{d c}^{*}=55.5$ \\
1.5 & 11 & 100 & $i_{d c}^{*}=55.5$ \\
2.0 & 11 & 110 & $i_{d c}^{*}=61$ \\
2.5 & 11 & 90 & $i_{d c}^{*}=50$ \\
3.0 & 10 & 90 & $i_{d c}^{*}=50$ \\
3.5 & 10 & 70 & $i_{d c}^{*}=39$ \\
4.0 & 9 & 70 & $i_{d c}^{*}=39$ \\
4.5 & 9 & 50 & $i_{d c}^{*}=27.75$ \\
5.0 & 8 & 50 & $i_{d c}^{*}=27.75$ \\
5.5 & 8 & 20 & $i_{d c}^{*}=i_{d c, \min }=22.2$ \\
6.0 & 7 & 20 & $i_{d c}^{*}=i_{d c, \min }=22.2$ \\
\hline
\end{tabular}


As shown in Fig. 5.11(a), MPPT controller behaves as expected by tracking the shaft speed reference. Generator voltage and frequency vary as wind speed changes. At $\mathrm{t}=0 \mathrm{~s}$, the system is exposed to rated wind speed of $12 \mathrm{~m} / \mathrm{s}$ under no-load condition. The SCIG produces the rated power of $20 \mathrm{~kW}$ at rated voltage of $460 \mathrm{Vrms}$ and frequency $60 \mathrm{~Hz}$. As shown in Fig. 5.11(b), since no load is connected, the dc-link current reference is initially set to $i_{d c}^{*}=i_{d c \text {, min }}$, which is enough for the CSI to produce the small ac-side current required by the filter capacitors to define the load voltage. However, because the wind power exceeds $P_{b a t}^{*}$, the $i_{d c}$ surpasses the $i_{d c}^{*}$ at $\mathrm{t}=$ $0.03 \mathrm{~s}$; hence, the dc-link current reference is switched to the $i_{d c}^{* *}$ required for the battery to absorb the entire generated power. At $t=0.5 \mathrm{~s}, 20 \%$ of the rated load is to be supplied, but the excess power still exceeds $P_{b a t}^{*}$; hence, $i_{d c}$ is still regulated at $i_{d c}^{* *}$. Very low modulation index $\left(m_{i}\right)$ is noticed before $\mathrm{t}=1 \mathrm{~s}$ because $i_{d c}^{* *}$ is much higher than what is required by the load during this period. From $\mathrm{t}=1 \mathrm{~s}$ to $\mathrm{t}=7 \mathrm{~s}$, the difference between the wind power and demand is below $P_{b a t}^{*}$; thus, the dc-link current is regulated at the $i_{d c}^{*}$, set according to the load; hence, modulation index is close to unity except after $\mathrm{t}=5.5 \mathrm{~s}$, when $i_{d c}^{*}$ is maintained at $40 \%$ of rated $i_{d c}$ even though the load is only $20 \%$ of the rated value. Since the dc-link inductor provides an energy buffer between the generator-side and load-side converters, load-side voltage and frequency are controlled irrespectively of wind speed variations. This task is achieved by the CSI controller, which maintains the load-bus voltage magnitude at $380 \mathrm{~V} / 220 \mathrm{Vrms}$ and frequency at $60 \mathrm{~Hz}$, with a maximum deviation of within $\pm 0.2 \mathrm{~Hz}$ during load change. During simulation period, the highest THD of the line voltage at the load bus is about $4 \%$ which is below the common permissible limit of 5\%. Similarly, the THD of line current is about $1 \%$ (implying a nearly sinusoidal current). Thus, a very high waveforms-quality is obtained at the load side with the help of the output Cfilter. As shown in Fig. 5.11(c), the battery bank either absorbs or delivers power according to the difference between wind power and load demand. Since the battery SoC is below the upper limit during charging, no power needs to be consumed by the dump load. From Fig. 5.11(b), one can notice that the dc-link current reference is adjusted gradually to the desired value because the dc choke will not allow a step change in the dc-link current. 

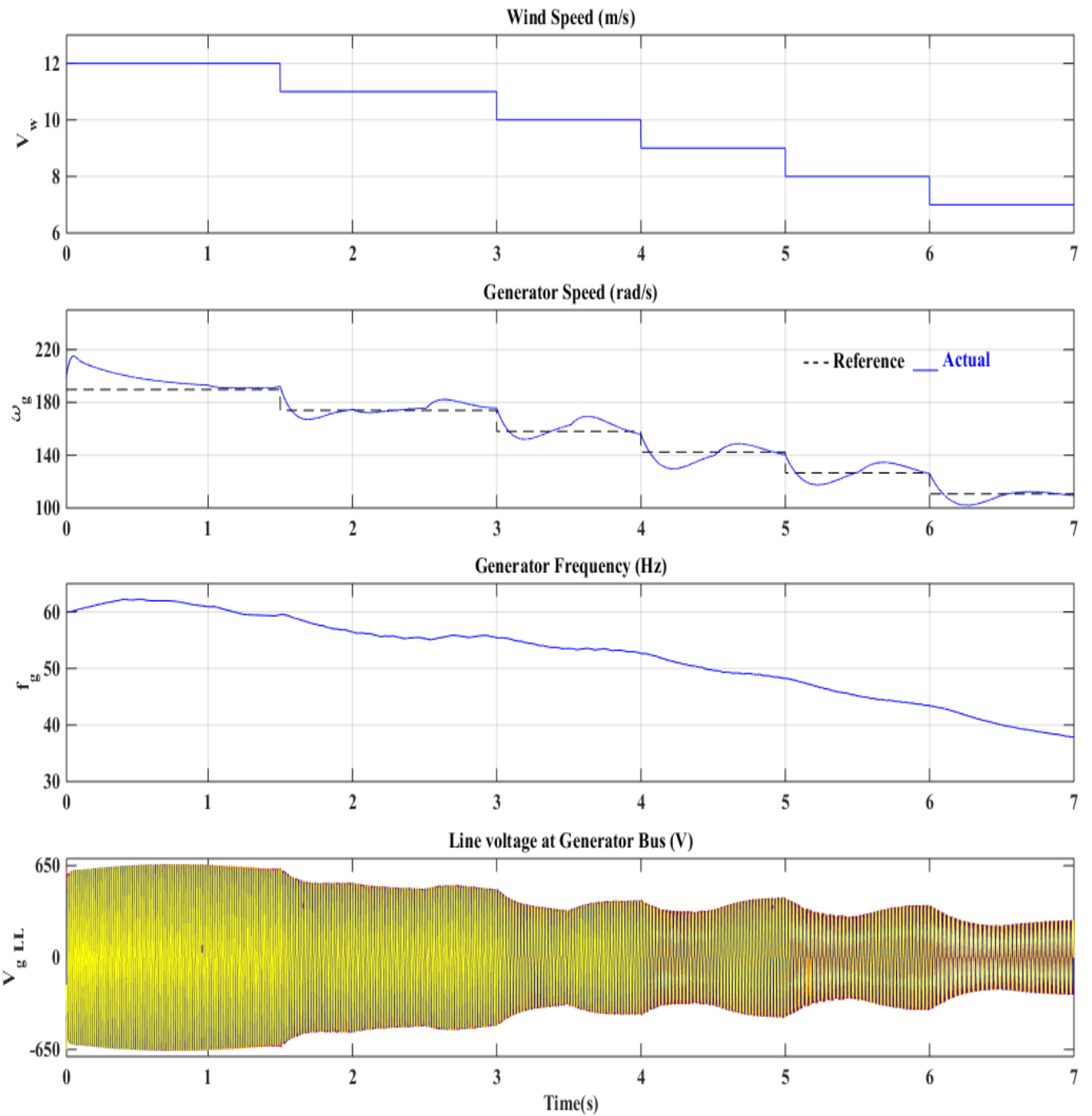

(a) Generator-Side Characteristics 

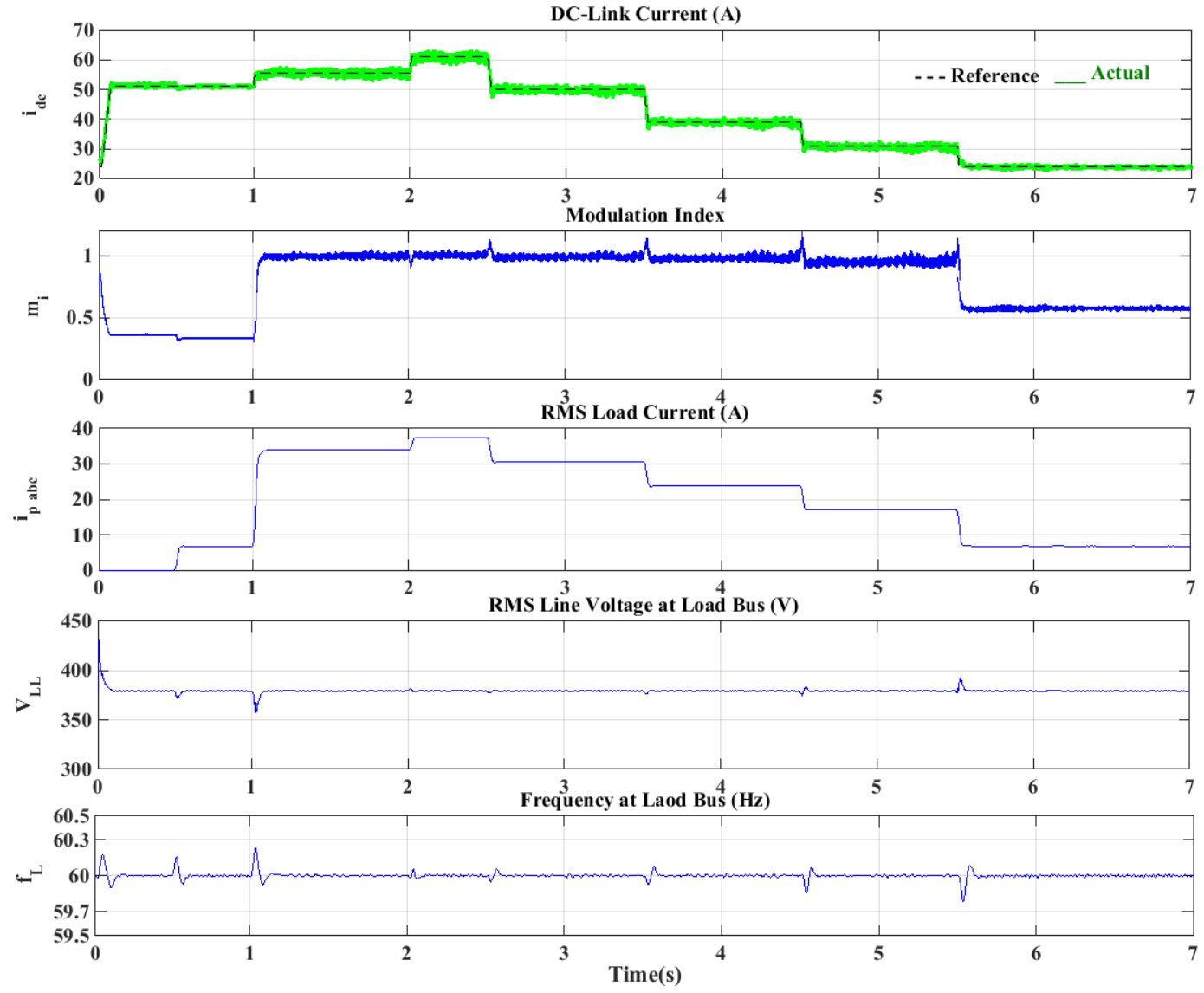

(b) DC-link and Load-Side Characteristics

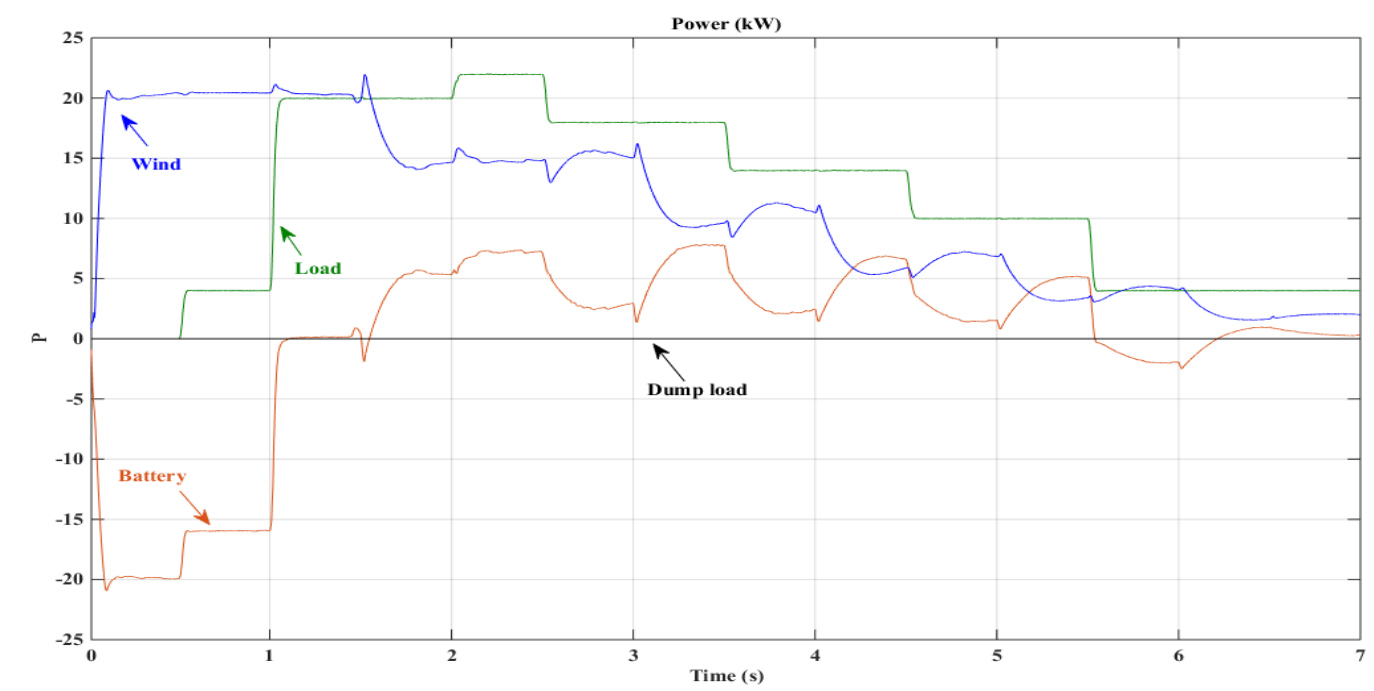

(c) Power Management

Fig. 5.11: System behavior under variable balanced load. 
One problem with using a diode rectifier as the generator-side converter is the resulting distortion in the stator current waveforms, leading to higher harmonic losses and torque ripples in the generator. This can be seen in Fig. 5.12(a) where a low generator inductance of $0.05 \mathrm{pu}$ is assumed. However, as mentioned in chapter 4 (section 4.4), low-power induction generator features relatively high inductance (i.e., $0.1-0.25 \mathrm{pu}$ ) which helps reducing the harmonics of the generator current as can be seen in Fig. 5.12(b) where a higher generator inductance of 0.15 pu is assumed. In this case, the generator experiences torque ripples within $10 \%$ versus $35 \%$ for the low generator inductance. It is worth mentioning that the quality of the generator current can be significantly improved by adding an external L filter on generator side, but at additional cost.
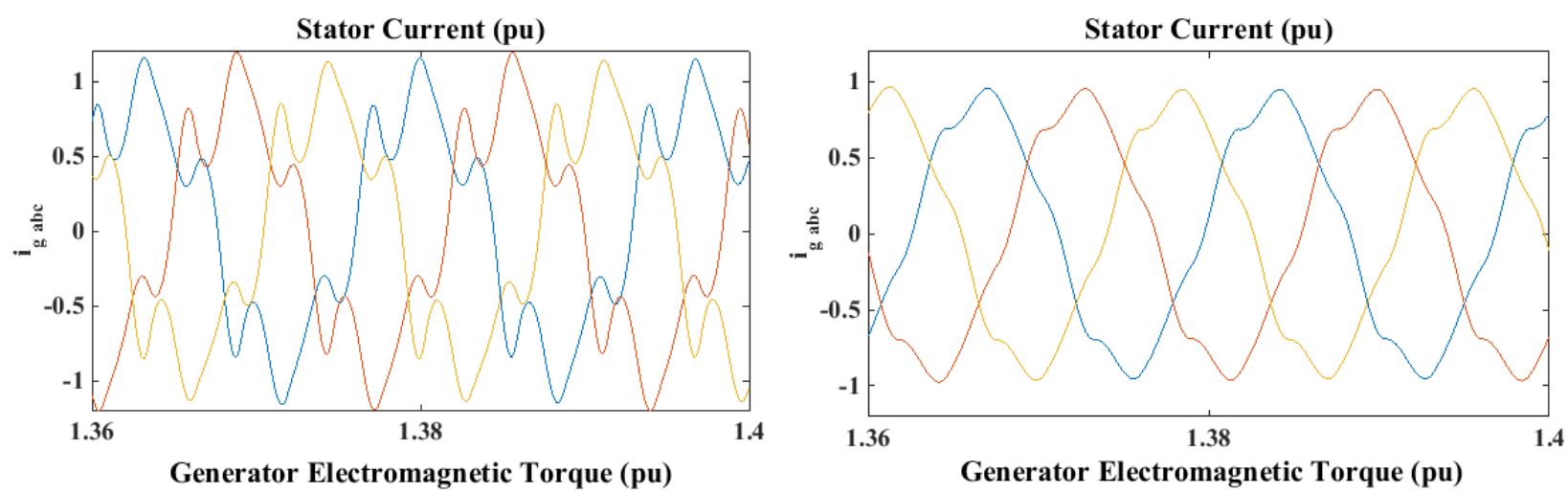

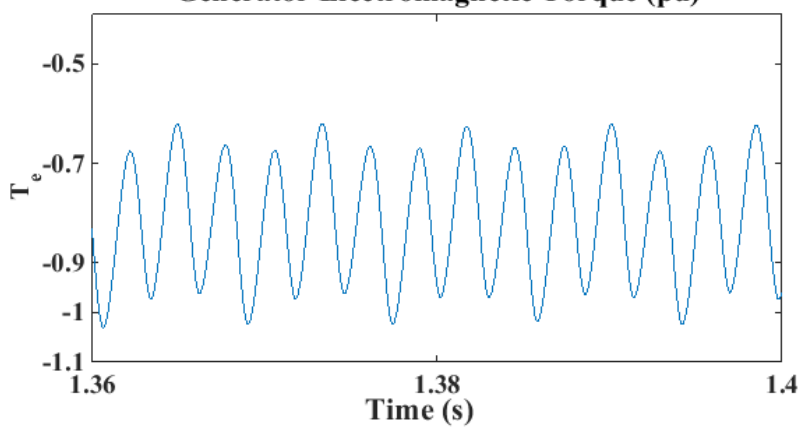

(a) Stator inductance $=0.05 \mathrm{pu}$

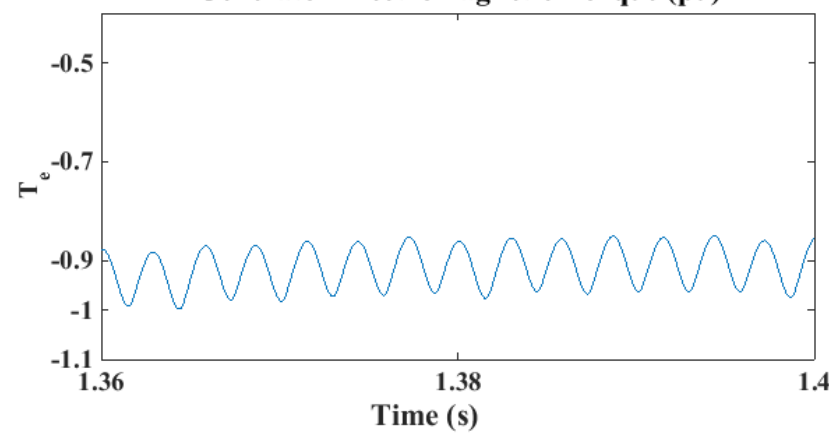

(b) Stator inductance $=\mathbf{0 . 1 5} \mathrm{pu}$

Fig. 5.12: Effect of generator inductance on stator current and electromagnetic torque.

Fig. 5.13 shows the simulation results for the case where the battery SoC is initially set very close to its upper limit (i.e., 75\%), wind is blowing at the rated speed (12 m/s) and the load is at $90 \%$ of its rated value. At start, the battery supplies the load and continues to deliver power until the wind turbine produces the whole demand at $t=0.16 \mathrm{~s}$. After this point, the battery is charged due to the availability of excess power. When battery SoC reaches its limit at $t=0.61 \mathrm{~s}$, the battery 
cannot be charged further; hence, the excess power is consumed by the dump load. The dc-link current is maintained at the desired value under all conditions.
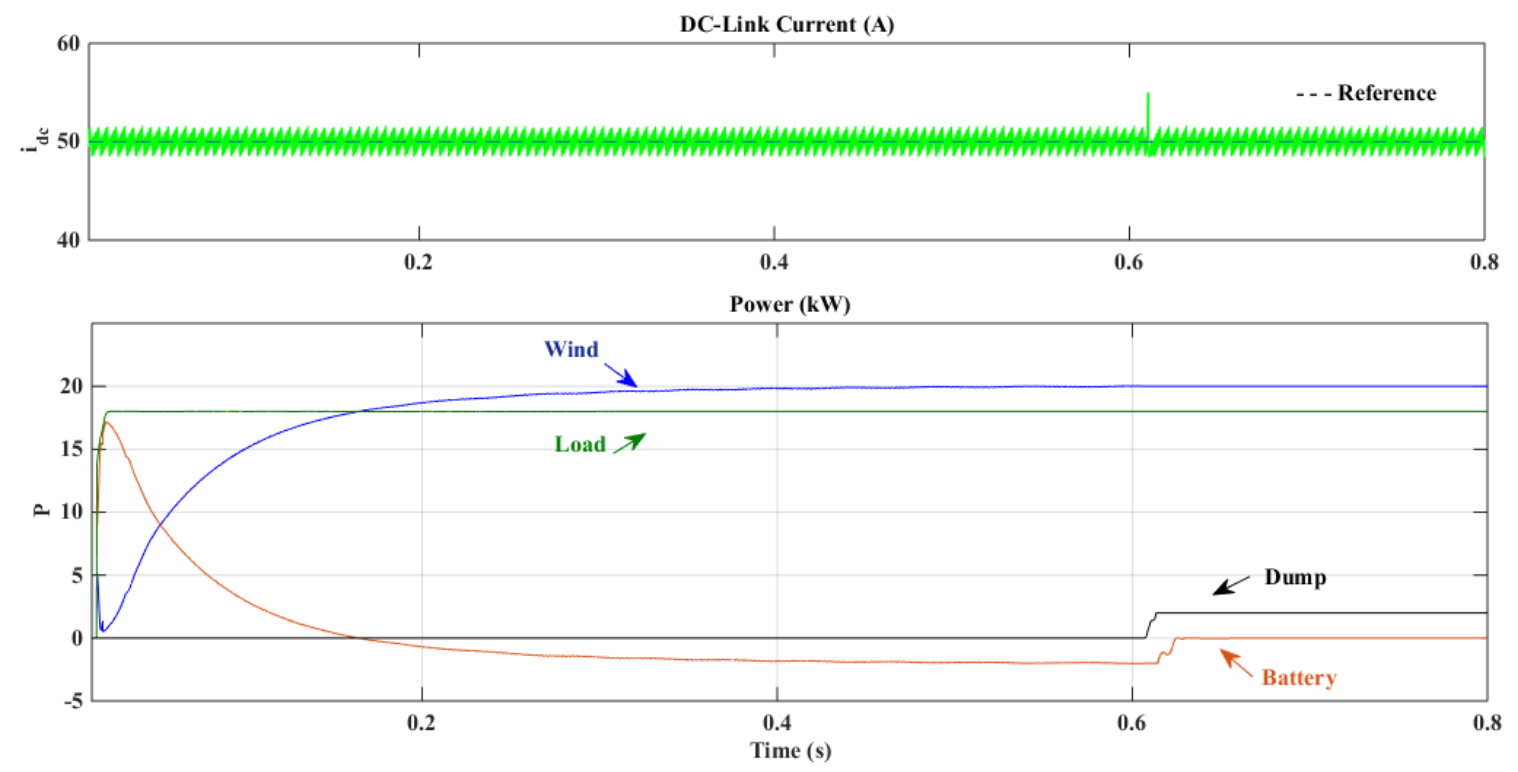

Fig. 5.13: Power management after battery SoC reaches upper limit.

\section{B) Variable Unbalanced Load}

The system is operated under the same conditions as in Table 5.3, but with unbalanced load. The rated values of three-phase load +ve seq active power, +ve seq reactive power, -ve seq $d$-axis power, and -ve seq $q$-axis power are: $P^{+}=20 \mathrm{~kW}, Q^{+}=10 \mathrm{kVar}, Q_{p d}^{-}=-5.08 \mathrm{kVar}$, and $Q_{p q}^{-}=-6.04 \mathrm{kVar}$, respectively; hence, the load unbalance factor is $35 \%$. In remote communities, the load unbalance factor can be much higher than 35\%, but this doesn't affect the generality of the load control scheme shown in Fig. 5.10. Simulation results are shown in Fig. 5.14. Since the dc-link current is controlled by the ES subsystem, the effect of unbalanced load on the WTG subsystem is negligible. Thus, the generator-side characteristics, under this case, are similar to those in the case of balanced load (see Fig. 5.11(a)). Load phase currents are not equal; hence, different modulation indices are produced for the three phases. Similar to the balanced-load case, the load-bus voltage magnitude is maintained at $380 \mathrm{~V} / 220 \mathrm{Vrms}$ and the frequency at $60 \mathrm{~Hz}$, with a maximum deviation of within $\pm 0.2 \mathrm{~Hz}$ during load change. As a result of employing $d q$ synchronous frame PI controllers, rms values of line voltages exhibits almost zero imbalance. VUF is varying with load. The highest value noticed for VUF is $0.2 \%$ which is far below the permissible 
limit of $1 \%$. During simulation period, $4.35 \%, 4.1 \%$, and $2.3 \%$ are the highest THDs detected in the line voltages $v_{a b}, v_{b c}$, and $v_{c a}$, respectively, which are still below the common permissible limit of 5\%. Similarly, the highest THDs of load currents are $1 \%, 0.73 \%$, and $0.81 \%$ for $i_{a p}, i_{b p}$, and $i_{c p}$, respectively, implying good sinusoidal currents.
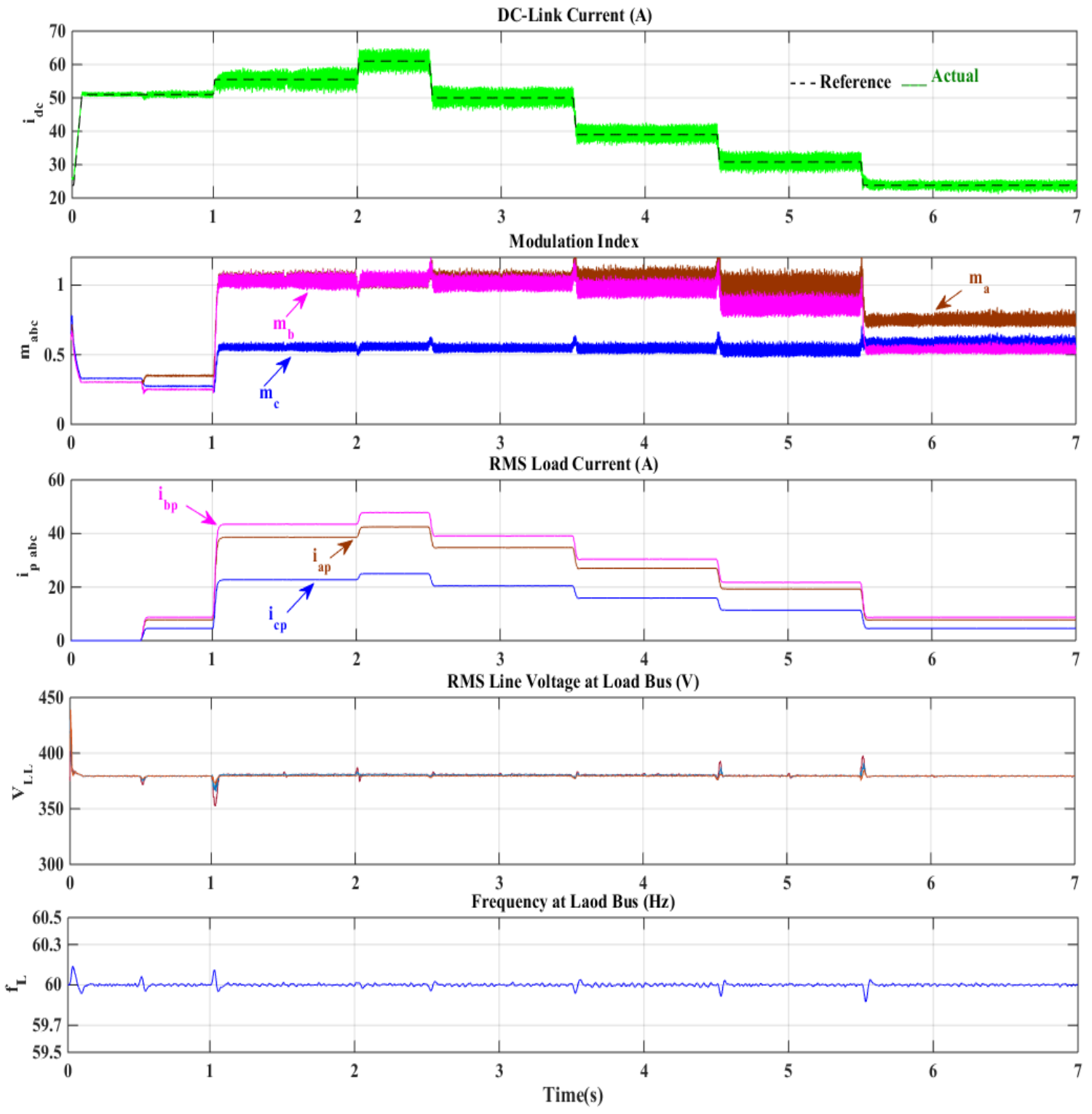

Fig. 5.14: System behavior under variable unbalanced load. 


\subsubsection{Effect of System Inertia and Frequency of Wind Speed Variation on MPPT}

The simulation results shown in Fig. 5.11(a) were obtained based on a turbulence-free wind speed profile. As described in chapter 4 (section 4.1), wind speed is a stochastic variable that can be described as the sum of average wind speed and the fluctuations about the average value. Wind speed fluctuations have a significant effect on achieving MPP. In MPPT speed controller, shown in Fig. 5.9 and described in subsection 5.4.1, the generator speed is controlled to track its optimal value at a given wind speed. Due to system inertia, there is a transient stage between any two steady-state generator speeds corresponding to two wind speed values. If the wind speed varies rapidly, the MPPT controller may not be able to track the optimum generator speed and hence the instantaneous maximum power will not be delivered. The effects of wind turbine inertia and rate of wind speed change on the MPPT operation and average generated power were investigated in [153]. It was shown that the average of the maximum power lost in a wind turbine increases with inertia and frequency of wind speed fluctuations. Fig. 5.15 shows the generator responses when the proposed system is run under two different low-pass filtered wind speed profiles generated by Von-Karman model at a height of 18 meters. The wind speed rate of change $\left(d v_{w} / d t\right)$ in Fig. 5.15(b) is higher than 2 times that in Fig. 5.15(a). As illustrated in the figure, the MPPT controller under wind speed profile with lower $d v_{w} / d t$ performs much better than in the case of higher $d v_{w} / d t$. The effect of system inertia is also shown in the figure for the two wind speed profiles. In Fig. 5.15(a), the optimum speed is tracked less precisely for $J=0.5 \mathrm{~kg} . \mathrm{m}^{2}$ than for $J=0.1 \mathrm{~kg} \cdot \mathrm{m}^{2}$. As a result, over the displayed period, the average of the power lost for $J=$ $0.5 \mathrm{~kg} \cdot \mathrm{m}^{2}$ is twice that for $J=0.1 \mathrm{~kg} \cdot \mathrm{m}^{2}$. Under a higher rate of wind speed change (Fig. 5.15(b)), the average power lost becomes worse for both inertia values; in particular, $J=$ $0.5 \mathrm{~kg} \cdot \mathrm{m}^{2}$ results in an average power reduction of $18 \%$ versus $4 \%$ for a lower rate of wind speed change (Fig. 5.15(a)). It should be mentioned that in order to respond to the fast change of wind speed in Fig. 5.15, the PI parameters of the MPPT controller are tuned under very high bandwidth. 

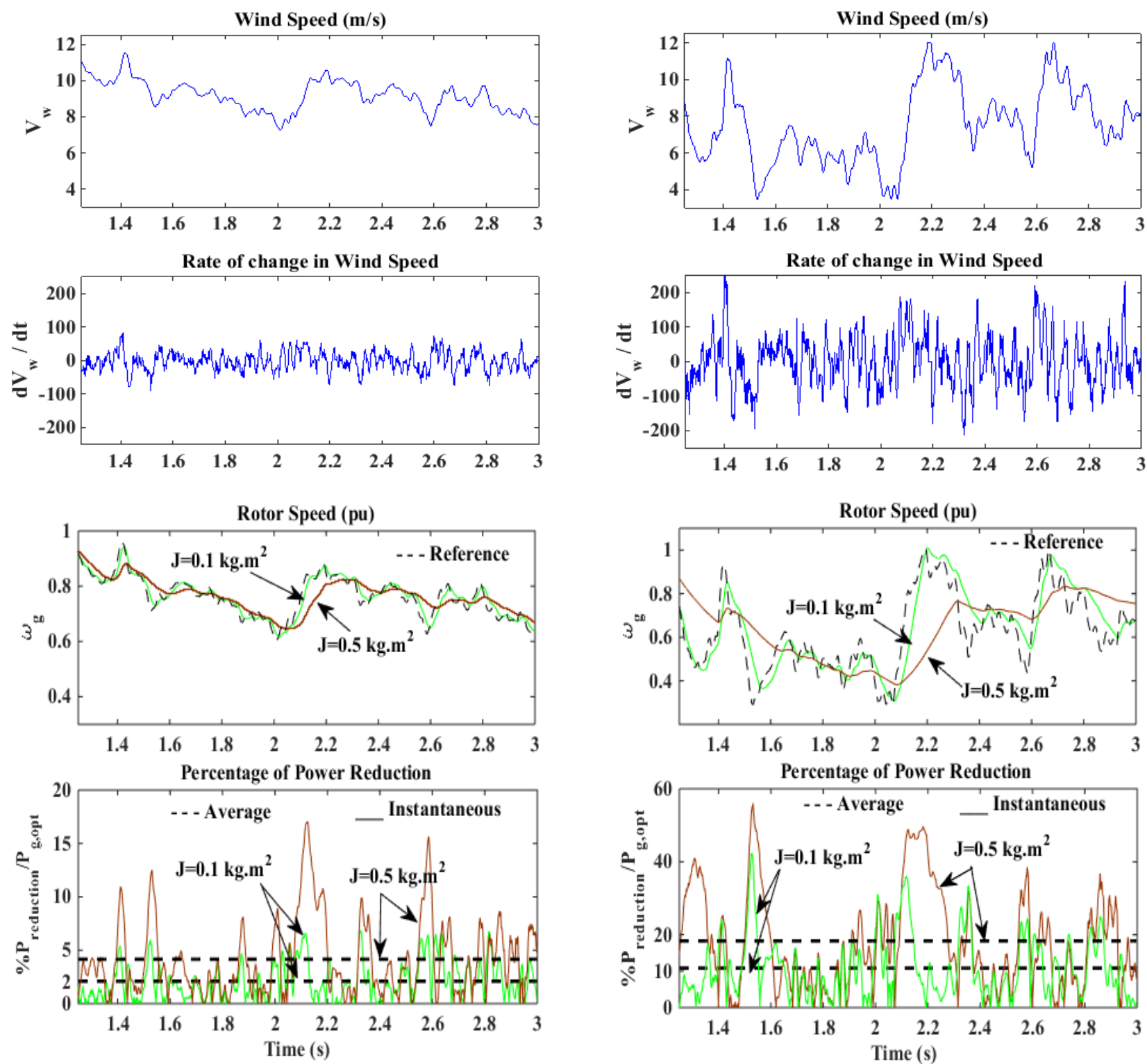

(a)

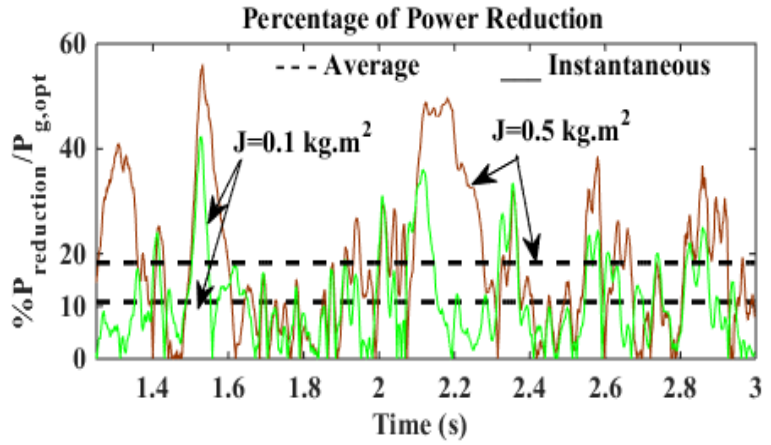

(b)

Fig. 5.15: Effects of system inertia and frequency of wind speed variation on MPPT.

\subsubsection{Performance of the Synchronous $d q$ Controller under Various Dynamics of Load}

In subsection 5.6.1, the system was examined under load profiles with same dynamic characteristics. In this subsection, Fig. 5.16 illustrates the performance of the synchronous $d q$ controller under load conditions with various dynamics (natural damping $(d)$ and oscillation frequency $\left.\left(\omega_{o}\right)\right)$ and steady-state characteristics, as given in Table 5.4. The nominal values of +ve 
seq active power, + ve seq reactive power, -ve seq $d$-axis power, and -ve seq $q$-axis power are: $P_{o}^{+}=20 \mathrm{~kW}, Q_{o}^{+}=10 \mathrm{kVar}, Q_{p d o}^{-}=-5.08 \mathrm{kVar}, \quad$ and $Q_{p q o}^{-}=-6.04 \mathrm{kVar}$, respectively . Because harmonics are not of interest in this subsection, the simulation responses shown here are obtained based on the average model of the system.

Table 5.4: Load parameters for simulation results of Fig. 5.16.

\begin{tabular}{cccccccc}
\hline $\begin{array}{c}\text { Time } \\
(\mathbf{s})\end{array}$ & $\begin{array}{c}\boldsymbol{d} \\
\left(\boldsymbol{s}^{-\mathbf{1}}\right)\end{array}$ & $\begin{array}{c}\boldsymbol{\omega}_{\boldsymbol{o}} \\
(\mathbf{r a d} / \boldsymbol{s})\end{array}$ & \% of $\boldsymbol{P}_{\boldsymbol{o}}^{+}$ & \% of $\boldsymbol{Q}_{\boldsymbol{o}}^{+}$ & $\%$ of $\boldsymbol{Q}_{\boldsymbol{p d o}}^{-}$ & $\%$ of $\boldsymbol{Q}_{\boldsymbol{p q o}}^{-}$ & $\begin{array}{c}\boldsymbol{i}_{\boldsymbol{d c}, \boldsymbol{r e f}} \\
(\mathbf{A})\end{array}$ \\
\hline 0 & 0 & 0 & 0 & 0 & 0 & 0 & $i_{d c}^{* *}=51$ \\
0.5 & 10 & 37 & 60 & 60 & 0 & 0 & $i_{d c}^{*}=33.3$ \\
1.5 & 5 & 75 & 30 & 30 & 50 & 50 & $i_{d c}^{*}=50$ \\
3.0 & 15 & 120 & 10 & 10 & 50 & 50 & $i_{d c}^{*}=55.5$ \\
\hline
\end{tabular}

Before connecting any load, the system operates at rated wind speed and the dc-link current is regulated at $i_{d c}^{* *}$, required for the battery to absorb the entire generated power. At $\mathrm{t}=0.5 \mathrm{~s}$, a balanced load is connected to the load bus. Therefore, no -ve seq voltage or current exists. The + ve seq $d q$ load currents oscillate at a frequency of about $37 \mathrm{rad} / \mathrm{s}$ before settling down at $0.9 \mathrm{~s}$ (i.e., $t_{s}=0.4 s$ ). The $d q$ load voltages experience very small undershoots and overshoots before settling at the desired values of $v_{c i d}^{+}=310.3 \mathrm{~V}(1 \mathrm{pu})$ and $v_{c i q}^{+}=0 \mathrm{~V}$. At $\mathrm{t}=1.5 \mathrm{~s}$, an unbalanced load is connected to the load bus; adding more +ve seq demand and introducing a -ve seq reactive power. At $t=3 \mathrm{~s}$, another unbalanced load is switched on. As can be seen from the figure, the $-\mathrm{ve}$ seq voltages are compensated by the synchronous $d q$ controller, and therefore the voltage imbalance caused by the unbalanced load is corrected. Only during transients, a disturbance can be noticed. Since $v_{\text {cid }}^{+}$is almost fixed throughout the simulation, the +ve and -ve seq powers follow the patterns of their associated currents. Throughout the simulation interval, except before $t=0.5$ $\mathrm{s}$, the dc-link current is regulated at $i_{d c}^{*}$, set according to the load. Fig. 5.17 shows the three-phase load currents and voltage (only peak portion) before and after connecting the unbalanced load at $\mathrm{t}$ $=1.5 \mathrm{~s}$. The ability of synchronous $d q$ control to achieve zero steady-state error can be clearly seen under balanced (before $\mathrm{t}=1.5 \mathrm{~s}$ ) and unbalanced (right before $\mathrm{t}=2.4 \mathrm{~s}$ ) load conditions. During load change, the voltage experiences transients within $\pm 5 \%$. 

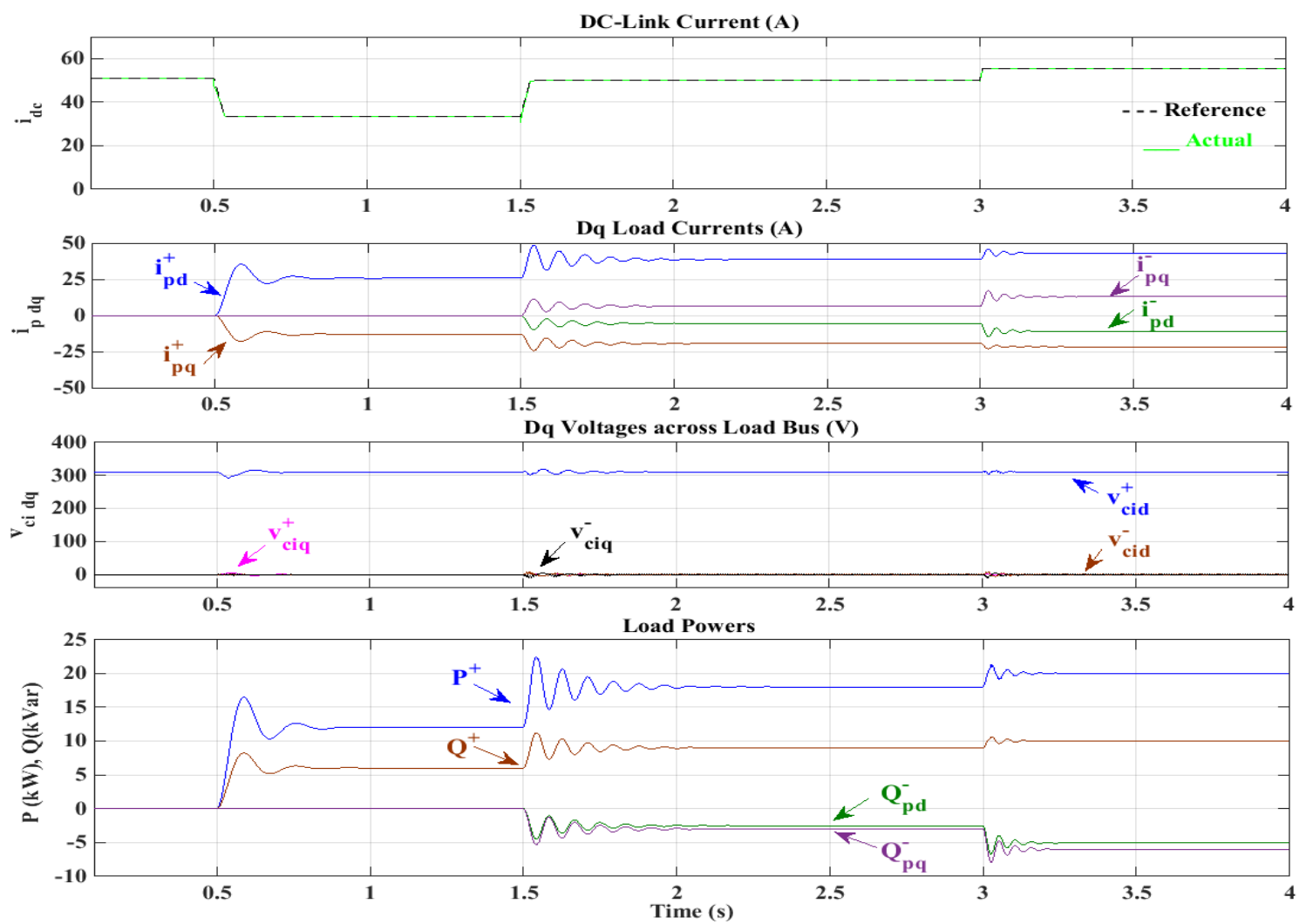

Fig. 5.16: Load characteristics under synchronous $d q$-frame controllers.

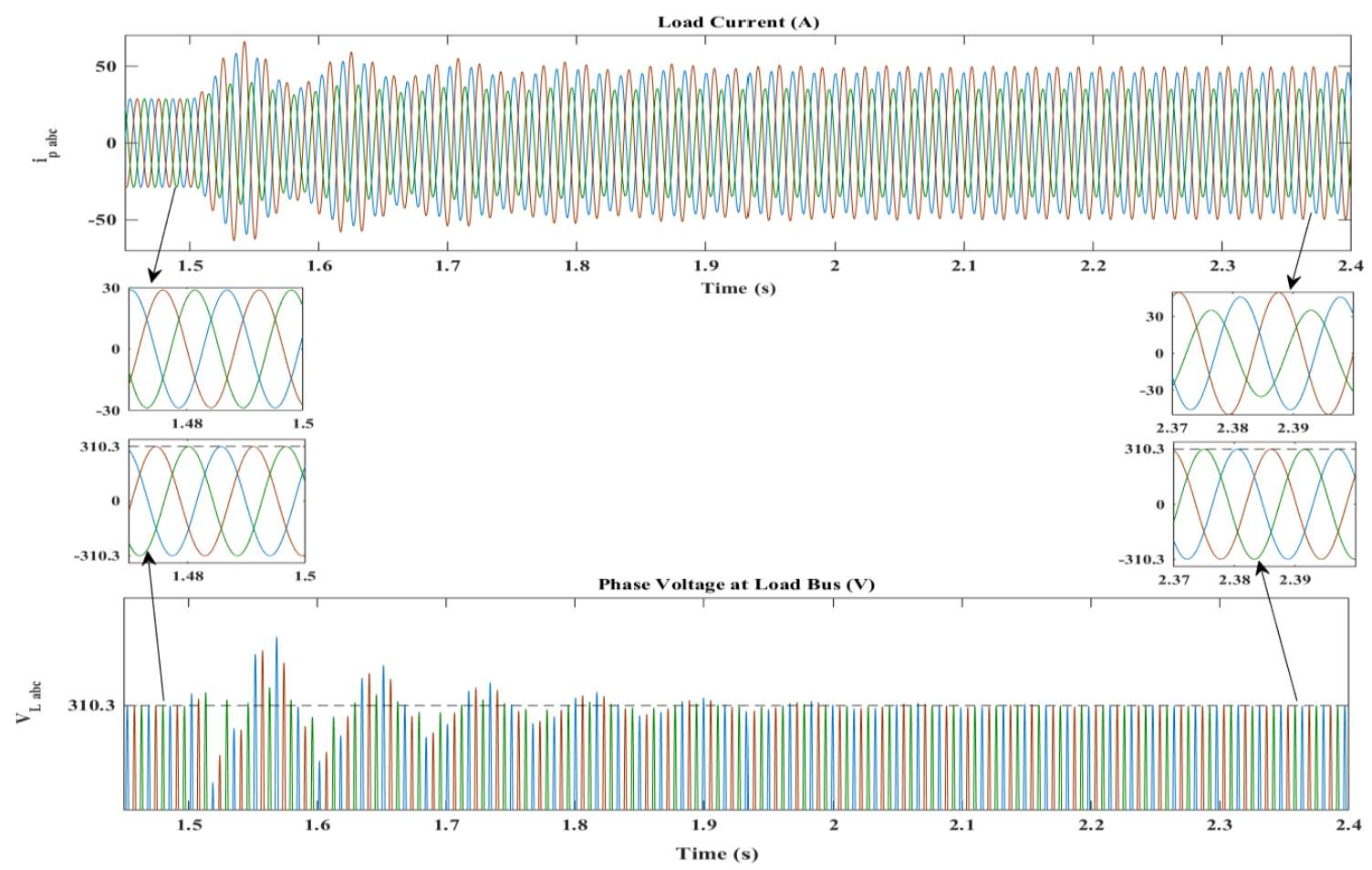

Fig. 5.17: Load-side three-phase voltages and currents under synchronous $d q$-frame controllers. 
Although synchronous $d q$-frame-based controllers, shown in Fig. 5.10, provide very satisfactory performance due to achieving zero steady-state amplitude and phase errors, the intensive computations, required to extract the symmetrical components from the measured asymmetrical voltages, complicates the control approach and introduce time delays in the feedback signal. A simpler control scheme with no time delay can be obtained by implementing stationary abc-frame PI controllers as shown in Fig. 5.18. In this scheme, the modulating signals of the CSI's three legs are separately produced to balance the voltage at load bus under unbalanced conditions. Nevertheless, three-phase stationary frame PI controllers, in general, result in steady-state amplitude and phase errors when regulating ac quantities [154]. This can be seen in Fig. 5.19 where a steady-state three-phase load voltage under a load condition given in Table 5.4 is illustrated. Although the stationary abc-frame PI controller provides very good voltage regulation under unbalanced load (see Fig. 5.19(a)), the phase voltages still exhibits some imbalance (see Fig. 5.19(b)). The VUF is 0.88\%. Moreover, Fig. 5.19(c) shows a phase shift between the regulated voltage and its reference. On the contrary, voltage responses regulated by the synchronous $d q$ controller are almost identical to the reference in magnitude and phase. The VUF is $0.19 \%$ which is much less than that of stationary frame PI controller and far below the permissible limit of $1 \%$.

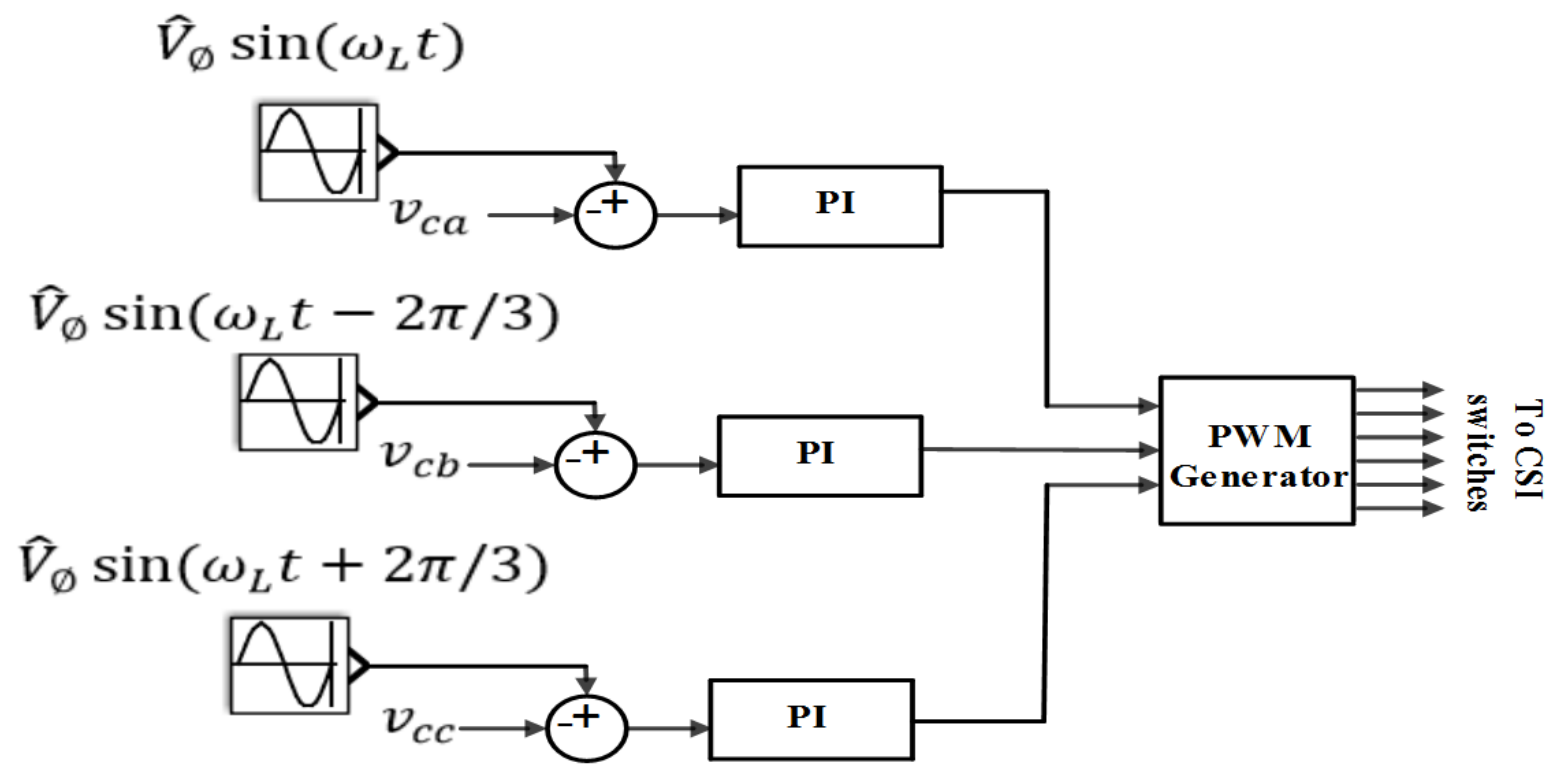

Fig. 5.18: Load-side stationary abc frame control scheme using PI controllers. 

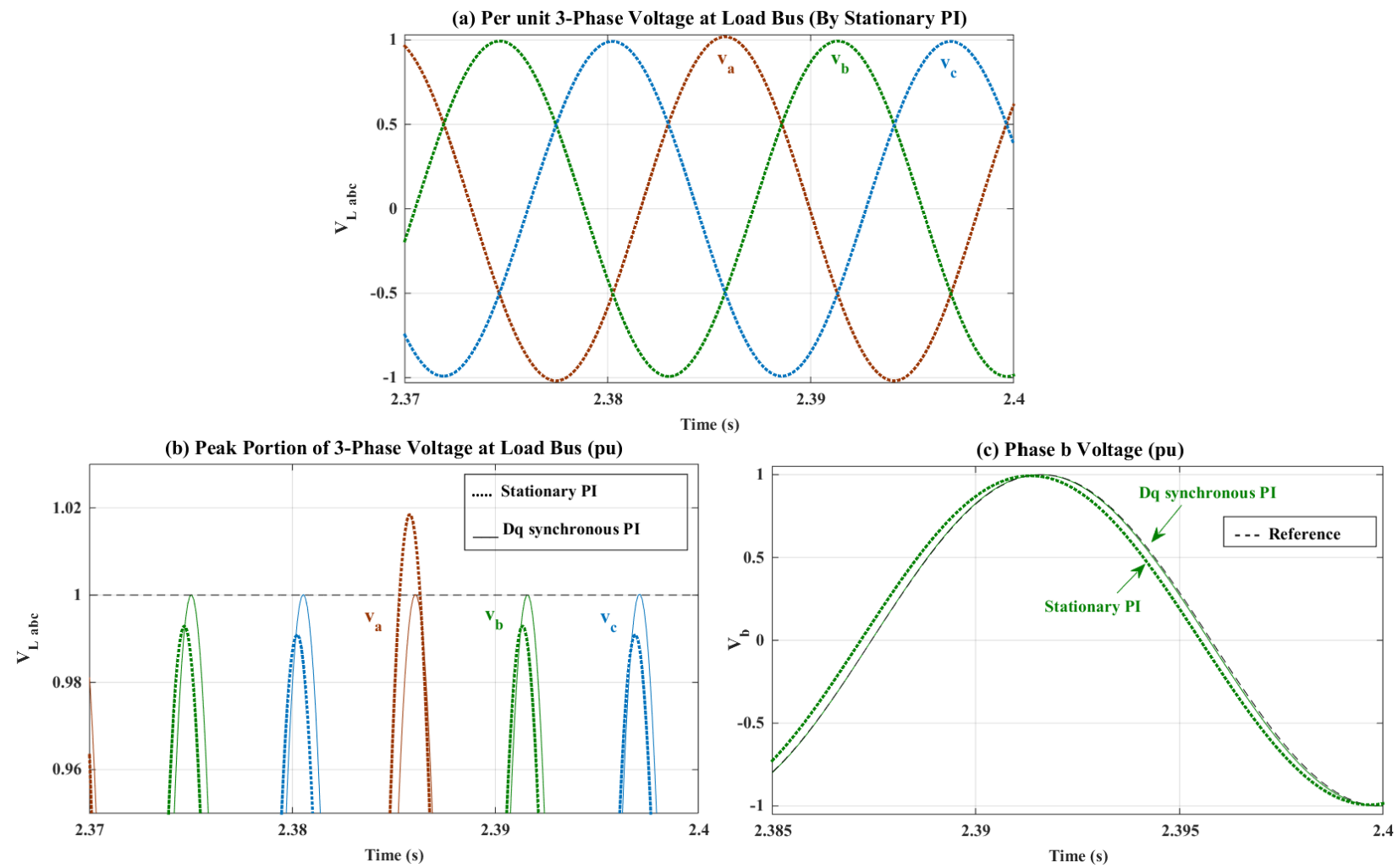

Fig. 5.19: Performance comparsion of abc-frame and $d q$-frame PI controllers.

\subsection{Fault Analysis of the CSI-based WECS}

As highlighted in chapter 1 (section 1.2.4), one of the advantages of CSI-based systems over VSI-based systems is that the dc-link current is directly regulated; hence, the ac-side current may not face a sharp rise during faults on the ac side of the inverter. This section studies the behaviour of the proposed system under different types of faults in the ac side of the inverter. In order to conduct this study, protection breakers, which typically open during faults to protect the inverter and the loads, are assumed to be deactivated. The neutral of the $\Delta / Y_{n}$ transformer is usually grounded for safety reasons. Three types of fault, i.e., Single Line to Ground (SLG), Line to Line (LL), and Three-Phase to Ground (TPG) faults are considered.

In Fig. 5.20, the Wind Turbine Generation (WTG) subsystem, the Energy Storage (ES) subsystem, and the CSI-Load subsystem are controlled by the MPPT controller, shown in Fig. 5.9, the dc-link current controller, shown in Fig. 5.3, and the load-side controller shown in Fig. 5.10, respectively. 


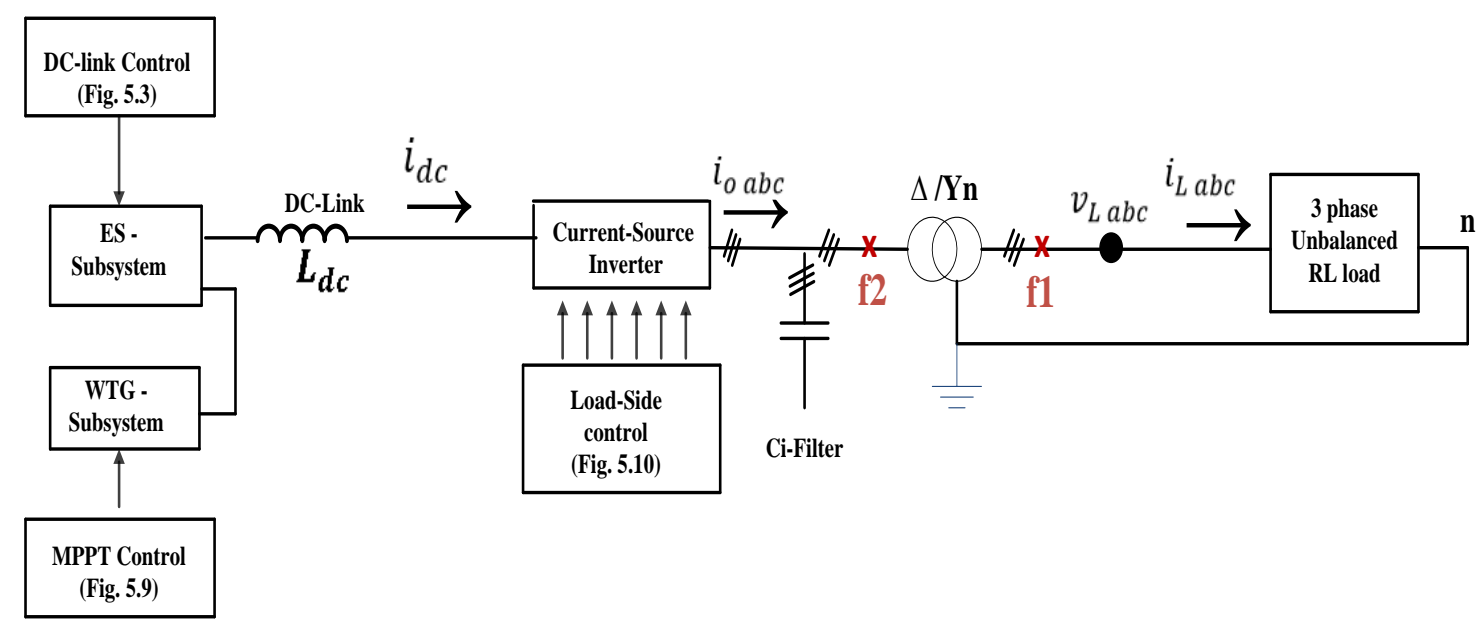

Fig. 5.20: Fault points on the proposed WECS.

Before applying a fault, the system is operating at a steady state operating point corresponding to rated wind speed and is supplying a 3-phase unbalanced RL load $(20 \mathrm{~kW}$ at $380 \mathrm{~V} / 220 \mathrm{~V}$, $\mathrm{pf}=0.89$ ). The values of resistances and inductances for the unbalanced load are $4.8 \Omega / 3.7 \mathrm{mH}$, $3.66 \Omega / 14.56 \mathrm{mH}$, and $6.93 \Omega / 3.57 \mathrm{mH}$ for phases a,b and c, respectively. Fig. 5.21(a) displays the system responses to an SLG fault applied to phase a on the secondary side of the transformer (point $\mathrm{f} 1$ in Fig. 5.20) from $\mathrm{t}=0.1 \mathrm{~s}$ to $0.2 \mathrm{~s}$. Due to the fault, voltage $v_{L a}$ drops to zero. The dclink current $\left(i_{d c}\right)$ experiences a small increase in the ripples which does not exceed $8 \%$ of the current. The dc-link current controller tracks the reference current in less than $15 \mathrm{~ms}$ after the fault is cleared at $0.2 \mathrm{~s}$. Since phase a is short circuited to ground, no current is supplied to phase a of the load during the fault. The CSI's unfiltered terminal current of phase a $\left(i_{o a}\right)$ undergoes over modulation, resulting in low order harmonics. Hence, the currents delivered to the other healthy load phases are no longer sinusoidal. This test clearly demonstrates the inherent over-current protection built in CSI that limits the currents on both dc and ac sides of the inverter. Moreover, the stable control of the dc-link current keeps the WTG subsystem at its normal operation, generating the rated wind power (i.e., 1 pu). During the fault, the load-side demand is reduced by $70 \%$ and hence the excess wind power is absorbed by the battery. Similar conclusions are made for LL and TPG faults, shown in Fig. 5.21(b) and (c), respectively. In all types of fault, the dc-link current is limited, resulting in a limited magnitude of the ac current delivered to the load. 

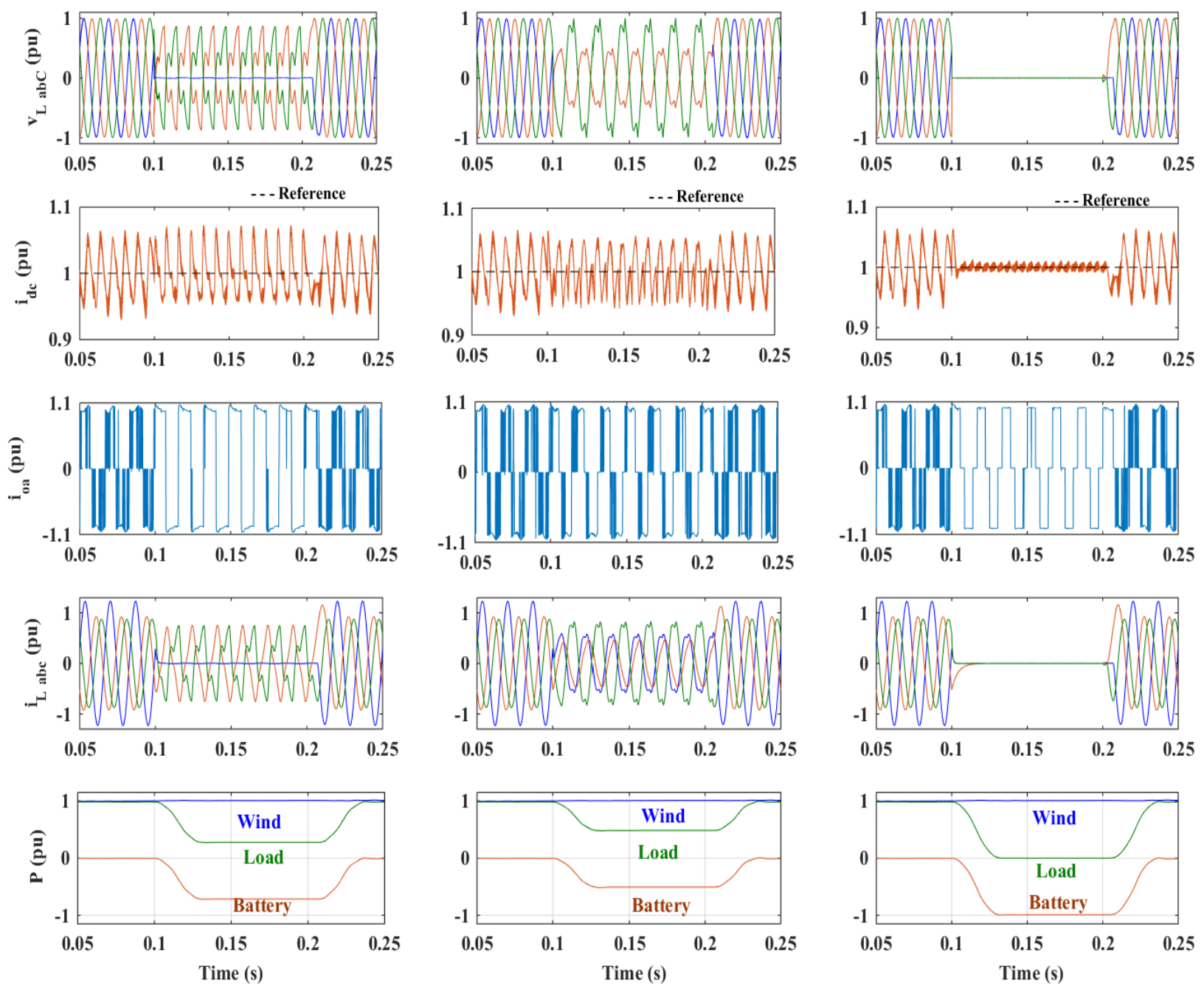

(a) SLG

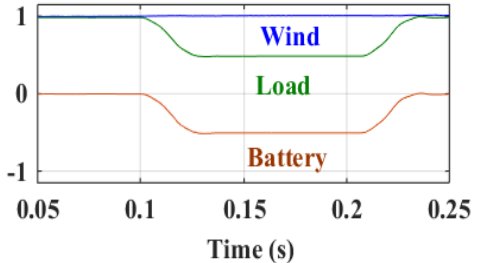

(b) LL fault

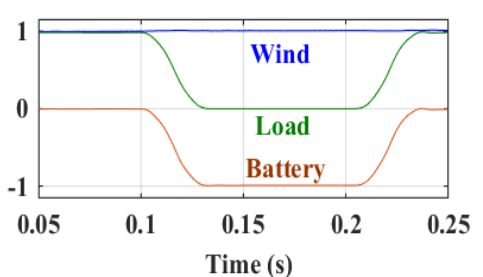

(c) TPG Fault

Fig. 5.21:System performance during (a) SLG (b) LL (c) TPG faults at secondary side of the $\Delta / Y_{n}$ transformer $\left(v_{L a b c}\right.$ : Load three-phase voltage, $i_{d c}$ : DC-link current, $i_{o a}$ : CSI phase-a unfiltered output current, $i_{L a b c}$ : Load three-phase current, and P: System powers).

Harmonic spectrums of the CSI terminal unfiltered and filtered currents $\left(i_{o a}\right.$ and $\left.i_{a}\right)$, before and during the SLG fault, are shown in Fig. 5.22. From Fig. 5.22(a) and (b), it is clear that no major low-order harmonics exists before fault and the CSI output capacitor filters the switching harmonics of $i_{a}$. On the other hand, as shown in Fig. 5.22(c) and (d), low-order harmonics are presented in $i_{o a}$ during fault and cannot be filtered by the CSI output capacitor as it is mainly designed for high-order harmonics attenuation. 

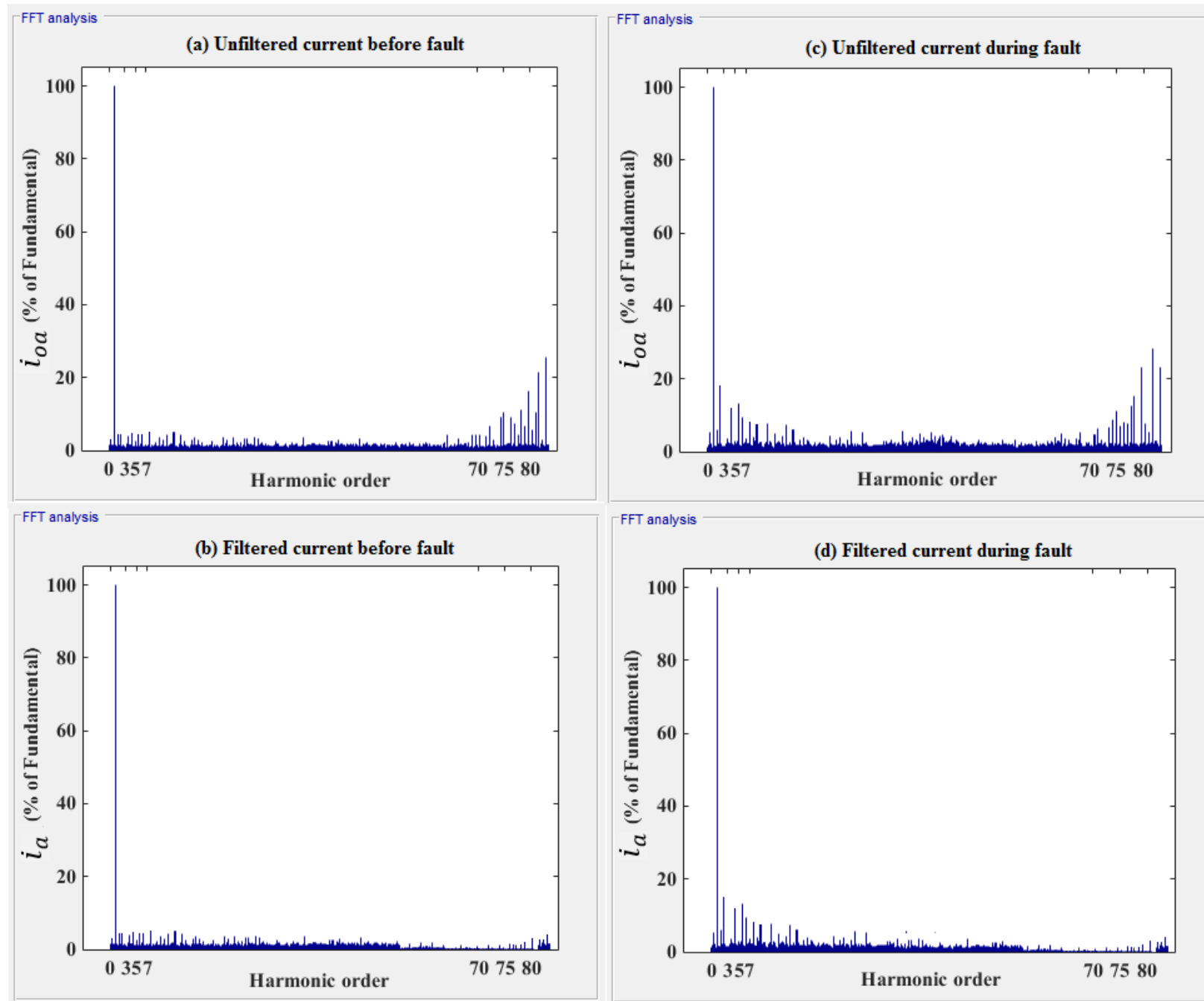

Fig. 5.22: Harmonic Spectrum of CSI's ac terminal and filtered currents before and during SLG fault.

Due to the linear relationships between the voltages on the primary and secondary sides of the Delta/Star transformer, system responses to LL fault applied on the primary side (point $\mathrm{f} 2$ in Fig. 5.20) are similar to those of SLG fault applied on the secondary side of the transformer. Likewise, system responses to three-phase (TP) fault applied on the primary side of the transformer are similar to those of TPG on the secondary side of the transformer; hence, these responses are not shown here.

In Fig. 5.23, the performance of a VSI-based standalone WECS and a CSI-based standalone WECS with comparable ratings (i.e., $20 \mathrm{~kW}$ ) are compared when an SLG fault is applied at $\mathrm{t}=$ $0.1 \mathrm{~s}$ on the secondary side of the transformer. In VSI-based WECS, the function of the dc-bus 
controller is to regulate the dc-side voltage, whereas in CSI-based WECS, the dc-bus controller regulates the dc-side current. From the figure, one can observe that the dc-side current of VSI (average value) is allowed to vary in a wide range and reach almost 7 pu (In practice, a current limiter is employed to limit this current). On the contrary, the dc-side current in CSI has very limited variations (within $1.08 \mathrm{pu}$ ).

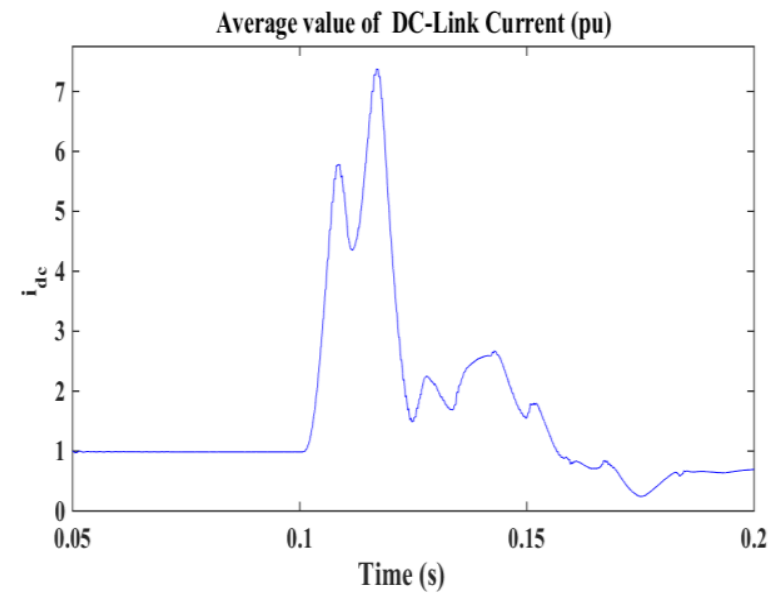

(a) VSI-based WECS

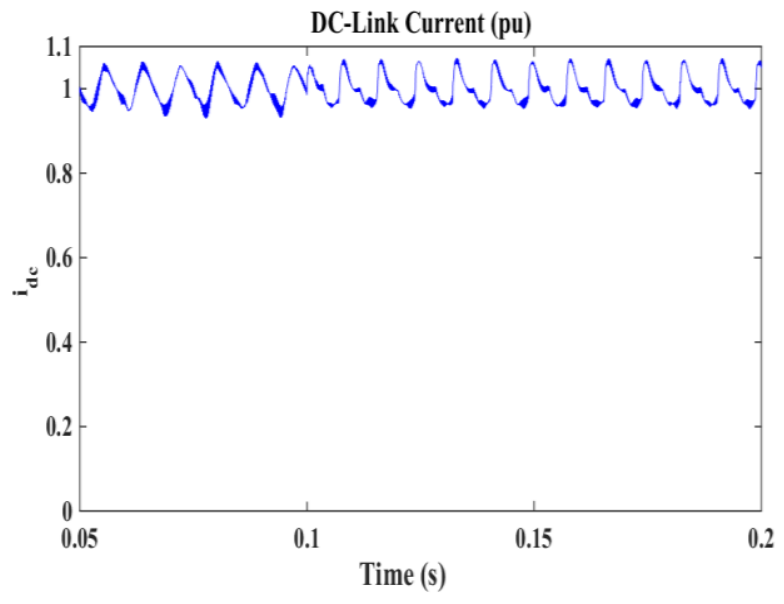

(b) CSI-based WECS

Fig. 5.23: Inverter dc-side current of VSI-and CSI-based WECS during an SLG fault.

\subsection{Dump Load-Less Version of the Proposed CSI-based WECS}

In the proposed CSI-WECS, shown in Fig. 5.1, the dc-link current is controlled using power management between the battery and dump load (see Fig. 5.3). The battery is charged or discharged based on the power mismatch between the wind generation and the demand. If the battery state-of-charge (SoC) reaches its upper limit, the excess power generated from the wind is dissipated in the dump load (see Fig. 5.13).

In this section, the system of Fig. 5.1 is modified by removing the dump load and implementing wind power curtailment through the generator-side converter when the combination of load and energy storage is not capable of absorbing the extra wind power. To achieve this objective, the MPPT/curtailment control scheme shown in Fig. 5.24 is implemented. 


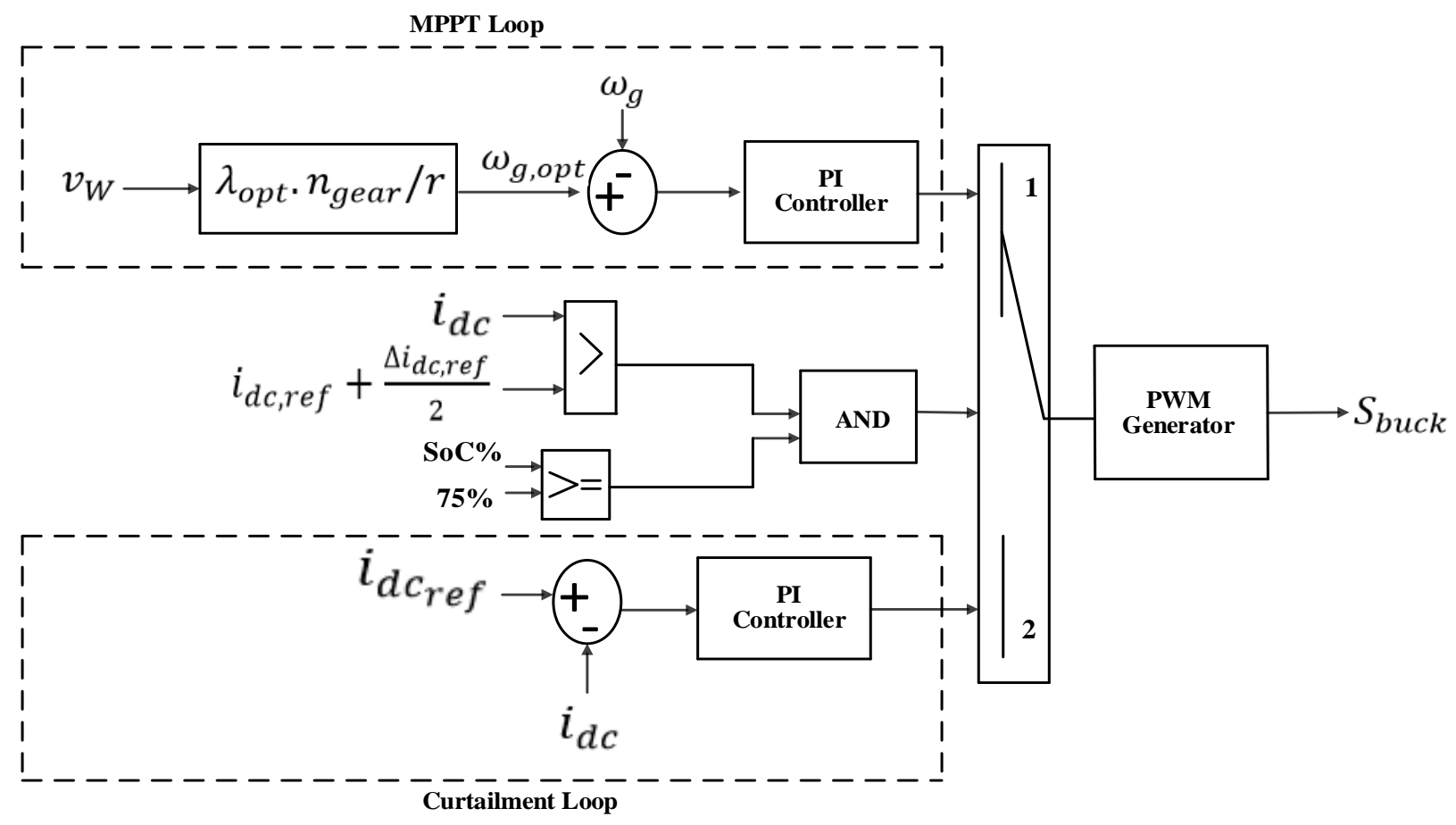

Fig. 5.24: MPPT/Curtailment Control Scheme.

The MPPT/Curtailment control scheme contains two control loops: generator-speed loop and dc-link current control loop. The generator-speed loop controls the generator in order to extract the maximum power in normal mode of operation (i.e., MPPT mode). This loop is identical with the MPPT control loop shown in Fig. 5.9 and described in subsection 5.4.1. If the wind power, generated under MPPT mode, exceeds the demand and the battery SoC reaches $75 \%$, the dc-link current controller, shown in Fig. 5.3 and described in subsection 5.3.1, will block the charging mode of the battery and hence $i_{d c}$ will exceed $i_{d c, r e f}$, assuming a new value depending on the level of supply-demand power mismatch. In order to prevent such a scenario, the function of buck converter, as shown in Fig. 5.24, is switched from shaft speed controller to current regulator so that the power absorbed form the generator is adjusted to maintain $i_{d c}$ at $i_{d c, r e f}$. The mechanism of switching between shaft speed control (MPPT control mode) and dc-link current regulation (curtailment control mode) is carried out by employing an automatic software switch, which by default assumes position 1, corresponding to MPPT mode. If battery SoC exceeds its upper limit, and $i_{d c}$ exceeds $i_{d c, \text { ref }}$ plus the permitted ripple, the switch assumes position 2, implying curtailment mode. It should be noted that the SoC condition for transition from speed control to 
current regulation is set to the upper limit ( $S o C \geq 75 \%$ ), assuming a very small mechanical system inertia and hence possibility of almost instantaneous transition. In practice, however, the system inertia should be considered by setting the SoC condition slightly less than its upper limit.

To study the performance of the MPPT/Curtailment control scheme for the dump load-less SCIG-CSI-WECS, the 20kW standalone WECS, described in Appendix A (Table A.2), is simulated under variable wind speed and unbalanced three-phase load, according to the wind speed and load profiles given in Table 5.5. The rated values of three-phase load powers are $P^{+}=20 \mathrm{~kW}, Q^{+}=$ $10 \mathrm{kVar}, Q_{p d}^{-}=-5.08 \mathrm{kVar}$, and $Q_{p q}^{-}=-6.04 \mathrm{kVar}$; hence, the load unbalance factor is $35 \%$. Simulation results are shown in Fig. 5.25. In order to verify the successful operation of the MPPT/Curtailment control scheme, the initial SoC is set very close to the upper limit of $75 \%$. At $\mathrm{t}=0 \mathrm{~s}$, the system is exposed to rated wind speed of $12 \mathrm{~m} / \mathrm{s}$. Since no load is connected, the dclink current reference is set to the $i_{d c}^{* *}$ required for the battery to absorb the entire generated power. At $\mathrm{t}=0.5 \mathrm{~s}, 20 \%$ of the rated load is to be supplied, but the excess power still exceeds $P_{b a t}^{*}$; hence, $i_{d c}$ is still regulated at $i_{d c}^{* *}$. From $\mathrm{t}=1 \mathrm{~s}$ to $\mathrm{t}=8 \mathrm{~s}$, the difference between the wind power and demand is below $P_{b a t}^{*}$; thus, the dc-link current is regulated at the $i_{d c}^{*}$. Throughout the entire simulation interval, except from $\mathrm{t}=4.7 \mathrm{~s}$ to $\mathrm{t}=6.07 \mathrm{~s}$, battery SoC is below $75 \%$. Therefore, the generator controller tracks the $\omega_{g, o p t}$ in order to achieve MPPT. The battery bank either absorbs or delivers power according to the difference between wind power and demand. When battery SoC reaches its upper limit at $\mathrm{t}=4.7 \mathrm{~s}$, the battery cannot be charged further; hence, the generator-side controller is switched to curtailment mode, where the shaft speed deviates from $\omega_{g, o p t}$ in order to limit the generated power to the demand level. At $t=5.5 \mathrm{~s}$, the load is decreased further and the curtailment controller readjusts generated power accordingly. The curtailment mode continues until $\mathrm{t}=6.07 \mathrm{~s}$ when the demand exceeds the generated wind power, forcing the battery to supply the deficit, and hence battery SoC drops below its upper limit. As a result, the generator controller reactivates the MPPT control mode. The dc-link current is maintained at the desired value under all conditions with current ripples within the typical limit of $15 \%$. It can be seen that the dc-link current ripple during the curtailment interval is slightly higher than those during MPPT intervals. 
Table 5.5: Wind speed and load conditions for simulation results of Fig. 5.25.

\begin{tabular}{cccc}
\hline Time $(\mathbf{s})$ & Wind speed $(\mathbf{m} / \mathbf{s})$ & \% of rated load & DC-link current reference (A) \\
\hline 0 & 12 & 0 & $i_{d c}^{* *}=51$ \\
0.5 & 12 & 20 & $i_{d c}^{* *}=51$ \\
1.0 & 12 & 100 & $i_{d c}^{*}=55.5$ \\
1.5 & 11 & 100 & $i_{d c}^{*}=55.5$ \\
2.0 & 11 & 110 & $i_{d c}^{*}=61$ \\
2.5 & 11 & 60 & $i_{d c}^{*}=33.3$ \\
3.0 & 10 & 40 & $i_{d c}^{*}=22.2$ \\
5.5 & 10 & 20 & $i_{d c}^{*}=i_{d c, \min }=22.2$ \\
6.0 & 10 & 70 & $i_{d c}^{*}=39$ \\
\hline
\end{tabular}
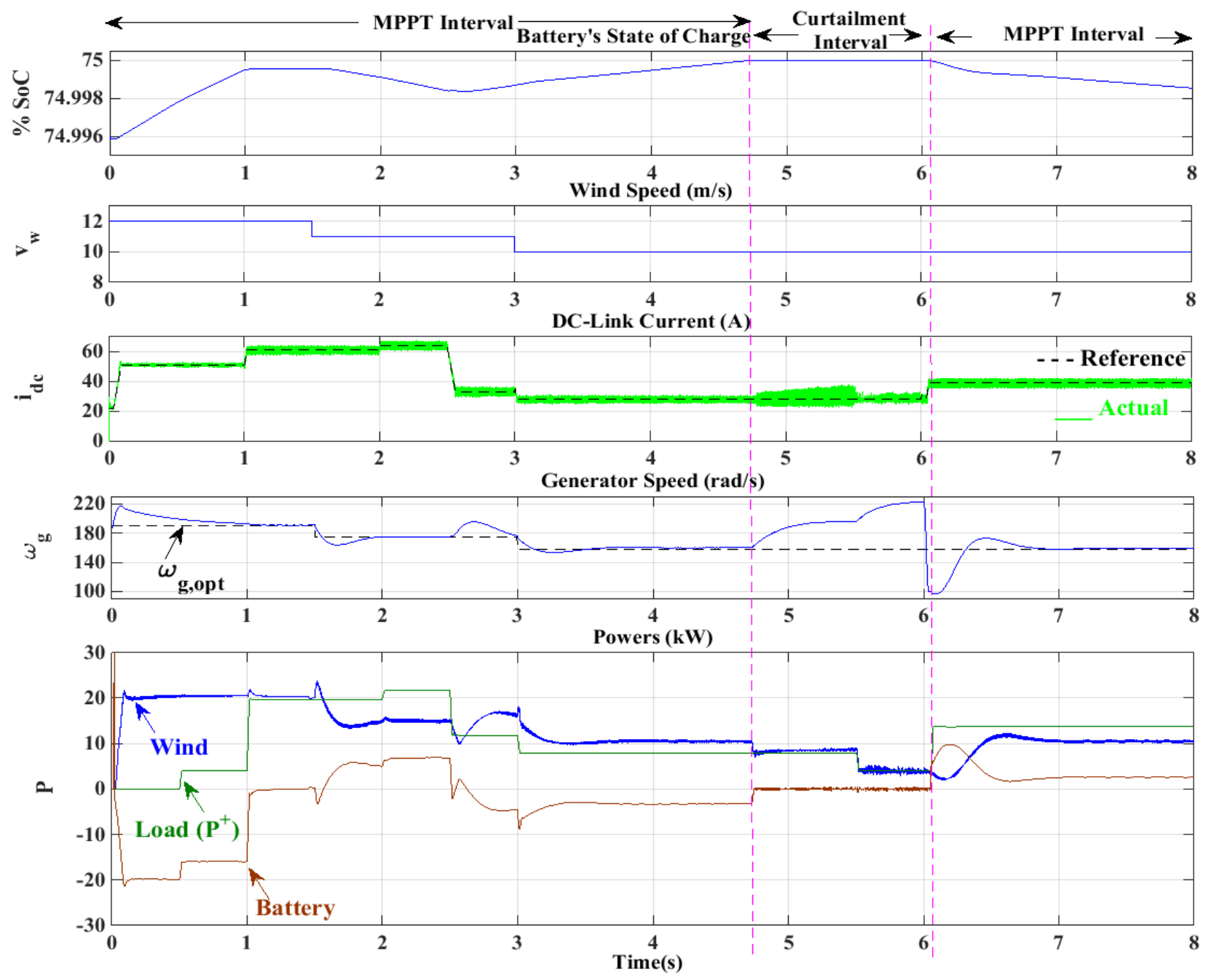

Fig. 5.25: System behavior under MPPT/Curtailment control. 
Because the wind power-shaft speed curve is nearly parabolic (see Fig. 4.3), there exists two operating shaft speeds (one lower and one higher than the optimum speed) at which wind power curtailment can be achieved. During curtailment interval, the buck converter regulates $i_{d c}$ by limiting the converter input current $\left(d_{b} i_{d c}\right)$ by reducing buck duty ratio $\left(d_{b}\right)$; hence, increasing shaft speed. As a result, as noticed in Fig. 5.25, the generator operates at the speed higher than the optimum speed. However, in order to reduce the mechanical stress on the shaft, it is desirable for the turbine shaft to operate at the lower speed that results in the targeted power. One way to achieve this is to use the generator-side converter (buck converter) to drive the machine into the low speed (obtained from wind power-shaft speed look-up table based on Fig. 4.3) that results in generating power as required by the load. However, this requires a prior knowledge of the short-term load demand which is time-varying and difficult to identify.

\subsection{Summary}

In this chapter, the closed-loop control system of the proposed CSI-based WECS was developed. The open-loop system is stable around operating points 1 and 3 but not 2; therefore, the objective of the closed-loop controller is to stabilize the system and track a class of desired references for the dc-link current and turbine speed, and control the voltage magnitude and frequency at the load bus.

Considering a fast and stable control for the dc-link current, the load-side and the generatorside converters are decoupled; therefore, the wind turbine system was decomposed into three subsystem, allowing the classical control theories for a SISO system to be applied.

An efficient control algorithm for the reduced H-bridge converter was developed to regulate the dc-link current through power management between battery bank and dump load. The fact that CSI's dc-link current can be reduced under light-load conditions was utilized to reduce dc-link and CSI power losses. However, this advantage could not be benefited from under certain conditions where a high dc-link current was required by the battery bank to absorb high excess power. The generator's shaft speed was controlled by the buck converter to extract maximum available wind power. The load-side voltage magnitude was regulated at the desired value by controlling the current-sourced inverter based on concept of positive and negative sequence decomposition. The 
parameters of the system PI controllers were tuned based on certain time and frequency domain specifications.

The performance of the closed-loop control system was verified under various wind speed as well as balanced/unbalanced load conditions, with different dynamic and steady-state characteristics. In all simulation results, a fast dc-link current control was demonstrated. Irrespective of load dynamic and steady-state characteristics, the synchronous $d q$ frame controllers maintained balanced voltage at the load bus, with limited disturbances during transients. Satisfactory performance of the MPPT controller under fixed and turbulent wind speed profiles was demonstrated. In the process, negative effects of wind speed rate of change and system inertia on MPPT were observed.

The performance of the proposed system under faults on the ac side was examined. The built-in inherent over-current protection that limits the currents on both dc and ac sides of the inverter, was demonstrated. This is an exclusive feature specific to CSI, thanks to the direct control of dc-link current.

A dump load-less version of the CSI-based system was proposed. The dump load was removed and the generator-side converter was used as a shaft-speed controller during MPPT mode and as a dc-link current regulator during curtailment mode. Successful operation of the MPPT/Curtailment controller under different wind speed and load conditions was demonstrated through simulation.

The core material of this chapter has appeared in the published journal paper [155] and has been included in the accepted conference paper [156]. 


\section{Chapter 6}

\section{Conclusions, Contributions, and Future Work}

In this thesis, a small-scale, standalone wind energy conversion system featuring SCIG, CSI and a novel storage integration scheme was proposed as an attractive renewable energy solution for off-grid communities.

In the following sections, the work presented in this thesis is summarized, the main contributions are highlighted and some items for future work are suggested.

\subsection{Summary and Conclusions}

This dissertation focuses on small-scale $(<100 \mathrm{~kW})$ wind turbines as very attractive renewable energy source for off-grid applications, especially in remote communities.

In chapter 1, a review of variable-speed wind energy conversion systems (WECSs) from generator and converter viewpoints was provided. Amongst the power converters which have been employed or have the potential to be employed in WECSs, voltage-sourced inverter (VSI)-based power electronic converters are the dominant topology in both large- and small-scale WECSs. On the other hand, current-sourced-inverter (CSI)-based power electronic converters have mainly been adopted in Megawatt-level on-grid WECSs. In order to assess the possibility of employing a CSI-based power electronic converter in off-grid low power wind turbines, a comparison between Pulse-width modulated CSI and VSI for small-scale standalone WECS, based on reliability, cost, efficiency, and protection requirements was conducted. Even though based on the comparison, CSI offers high potentials for small-scale off-grid WECS, its performance in such an application has never been investigated. Therefore, CSI-based power converter was selected as the base of this research. A brief review on the energy storage technologies that are feasible for wind energy integration was also conducted. As a low-cost mature storage technology, offering satisfactory performance, lead acid battery was selected for storage purposes.

Motivated by lack of a comprehensive and convincing approach to selection of the right generator for a standalone wind turbine, a thorough study, considering all possible options, was conducted in chapter 2. Amongst the different generator types considered in the study, three wind generator configurations, namely geared-drive squirrel-cage induction generator (geared-SCIG), 
direct-drive permeant-magnet synchronous generator (gearless-PMSG) and direct-drive permanent-magnet induction generators (gearless-PMIG) were identified as the most promising technologies for standalone wind turbines. Therefore, the three configurations were qualitatively compared with one another in terms of reliability, cost, efficiency, excitation requirements, control simplicity, construction simplicity, and noise level. The system based on geared-SCIG was shown to be the most appropriate scheme for a small-scale standalone WECS, supplying a remote area.

In chapter 3, the three CSI-based topologies, validated in the literature for on-grid WECS, were evaluated for off-grid WECS application. The first topology (i.e., diode bridge rectifier - PWM CSI) provides no control over generator and dc-link current; hence, it is not applicable for an offgrid WECS. The second topology (i.e., diode bridge rectifier - buck converter - PWM CSI) makes the generator control achievable. The main drawback of this topology is that it causes high harmonic distortion in the generator stator winding current. This drawback is avoided by employing current-sourced rectifier in the third topology (i.e., back-to-back PWM CSC), leading to considerable improvement in the generator performance, but at a higher cost and with higher complexity in control. As a simple and low-cost configuration, offering satisfactory performance, the second topology (i.e., diode bridge rectifier - buck converter - PWM CSI) was selected as the base for further study in this research. The duality of CSI and VSI topologies was taken advantage of to come up with a novel scheme for the integration of a battery-based energy storage system with the proposed CSI-based WECS. An H-bridge, with reduced number of switches and diodes, was employed as the interfacing converter. In the proposed system, the dc-link inductor is shared among the generator-side, storage-side and load-side converters, resulting in reduction of the system size, weight and cost.

Chapter 4 focused on developing the dynamic mathematical model of the proposed CSI-based wind turbine system. Detailed models of the system components were presented. A reduced-order generic load model suitable for balanced/unbalanced loads was developed. By combining the state equations of the system components, as well as the generic load model, the overall model of the system was described by seven inputs, six outputs, and twenty state variables. A small-signal model of the system was developed around three operating points. The eigenvalue analysis of the small-signal model showed that the open-loop system is locally stable around operating points 1 and 3, but not 2. Based on Gramian matrices, the system was found completely controllable at the 
three operating points, completely observable at operating points 1 and 3, but not completely observable at operating point 2.

Chapter 5 presented the design of the closed-loop control system. An efficient control algorithm for the reduced $\mathrm{H}$-bridge converter was developed to regulate the dc-link current, considering the minimum dc-link current requirements for both generator and load sides. The generator shaft speed was controlled to extract maximum available wind power. The load-side voltage was regulated at the desired value by synchronous $d q$ frame controllers, resulting in zero-steady-state error. The parameters of the PI controllers were tuned using Matlab/Simulink control tools based on certain time- and frequency-domain specifications. The performance of the closed-loop control system was verified under various wind speed and balanced/unbalanced load conditions of different dynamic and steady state characteristics. The performance of the proposed system under ac-side faults was also examined. The inherent over-current protection capability, built in CSI, that limits fault currents on both dc and ac sides of the inverter, was demonstrated. Finally, a dump load-less version of the CSI-based system was proposed and the successful operation of the MPPT/Curtailment controller was demonstrated.

\subsection{Contributions}

The main contributions of this research are as follows:

1) A critical analytical study of standalone wind energy conversion systems from generator viewpoint was conducted. This study has resulted in a journal publication [102] based on the review of 148 references, and serves as an excellent source of information and a helpful guide for researchers and practitioners involved in standalone wind turbine systems.

2) A small-scale, standalone wind energy conversion system featuring SCIG, CSI and a novel energy storage integration scheme was proposed. The dc-link inductor shared by three power electronic converters was systematically designed. Switching and average Simulink models of the system were developed.

3) An efficient power management algorithm to maintain supply-demand balance through direct control of the dc-link current was developed. The fact that the CSI's dc- 
link current can be reduced at light load was utilized to improve the dc-link and CSI efficiencies.

4) A reduced-order generic load model suitable for balanced/unbalanced load conditions was developed. The model is capable of emulating actual loads of different transient and steady-state characteristics and is appropriate for simulation studies of stability and dynamic performance of a standalone system.

\section{5) A dump load-less version of the developed CSI-based WECS was proposed.}

The work presented in this thesis has been published in [102],[155] and [156].

\subsection{Future Work}

The following items are suggested for future research.

\section{1) Experimental Verification}

A laboratory experimental platform of the proposed wind energy conversion system can be set up and used to verify the validity of the simulation results.

\section{2) A transformer-less version of the proposed CSI-based WECS.}

Although the Delta/Star isolation transformer provides a zero-sequence current path for unbalanced load, it is bulky and costly. In order to eliminate the need for the transformer, a path for the load neutral current must be provided by other means. A simple and low-cost solution was implemented in [157] for a CSI-based grid connected PV system by connecting the neutral terminal of the grid to the midpoint of the PV-side smoothing dc capacitor. For the proposed WECS, however, such a configuration will generate significant voltage ripple in the generatorside dc capacitor, causing more toque ripples and reducing the quality of the generated power.

\section{3) Sensor-less control of SCIG in the proposed WECS}

The MPPT control loop requires an anemometer to measure wind speed and a position sensor to measure the rotor speed. However, mechanical sensors increase the capital cost, as well as maintenance costs, and reduce the reliability of the turbine system. A sensor-less control algorithm for the SCIG-WECS will bring great benefits to the system. 


\section{Appendix $A$}

\section{Parameters and Operation Conditions of the Systems used in Simulation}

Table A. 1 and Table A. 2 give the parameters and operating conditions for the $30 \mathrm{~kW}$-CSC-based WECS and the 20kW-CSI-based WECS, respectively.

Table A.1: Parameters and operating condition of the 30kW-CSC-SCIG WECS.

\begin{tabular}{|c|c|c|c|}
\hline Requirement & Topology 1 & Topology 2 & Topology 3 \\
\hline Wind Turbine & \multicolumn{3}{|c|}{$\begin{array}{c}\text { Cut-in wind speed }: 5 \mathrm{~m} / \mathrm{s} \\
\text { Rated wind speed : } 12 \mathrm{~m} / \mathrm{s} \\
\text { Cut-out wind speed }: 20 \mathrm{~m} / \mathrm{s} \\
\text { Rated power }=30 \mathrm{~kW}\end{array}$} \\
\hline Gearbox & \multicolumn{3}{|c|}{$\begin{array}{c}3 \text { stages } \\
\text { Ratio: } 5: 1 \text { per stage }\end{array}$} \\
\hline Generator & \multicolumn{3}{|c|}{$\begin{array}{c}\text { Squirrel cage Induction } \\
30 \mathrm{~kW}, 320 \mathrm{~V}, 4 \text { pole }, 60 \mathrm{~Hz}, 1812 \mathrm{rpm}, \\
63 \mathrm{~A}, 158 \mathrm{~N} \cdot \mathrm{m}, \mathrm{pf}=0.86\end{array}$} \\
\hline $\begin{array}{l}\text { No-Load Excitation } \\
\text { Capacitor }\end{array}$ & $400 \mu \mathrm{F}(0.45 \mathrm{pu})$ & $400 \mu \mathrm{F}(0.45 \mathrm{pu})$ & None \\
\hline Input filter $\left(C_{r}\right)$ & None & None & $180 \mu \mathrm{F}(0.2 \mathrm{pu})$ \\
\hline $\begin{array}{l}\text { Generator-side } \\
\text { converter }\end{array}$ & Diode bridge rectifier & $\begin{array}{l}\text { Diode bridge rectifier } \\
+\mathrm{dc} / \mathrm{dc} \text { buck converter, } \\
f_{s}=2.1 \mathrm{kHz}\end{array}$ & $\begin{array}{l}\text { PWM- IGBT-CSR } \\
f_{s}=5.1 \mathrm{kHz}\end{array}$ \\
\hline DC Choke & $18 \mathrm{mH}(2.3 \mathrm{pu})$ & $6.2 \mathrm{mH}(0.8 \mathrm{pu})$ & $3.2 \mathrm{mH}(0.41 \mathrm{pu})$ \\
\hline Rated dc current & $84 \mathrm{~A}$ & $84 \mathrm{~A}$ & $84 \mathrm{~A}$ \\
\hline Inverter & \multicolumn{3}{|c|}{ PWM-IGBT-CSI, $f_{s}=5.1 \mathrm{kHz}$} \\
\hline Output Filter $\left(C_{i}\right)$ & \multicolumn{3}{|c|}{$125 \mu \mathrm{F}(0.14 \mathrm{pu})$} \\
\hline Rated Load & \multicolumn{3}{|c|}{ 3-phase balanced RL load $(33.5 \mathrm{kVA}$ at $380 \mathrm{~V} / 220 \mathrm{~V}$, pf $=0.89)$} \\
\hline
\end{tabular}


Table A.2: Parameters and rated operating conditions of the 20kW-CSI-SCIG-WECS.

\begin{tabular}{|c|c|c|c|}
\hline \multicolumn{4}{|c|}{ Turbine and drive train } \\
\hline$P_{m}=23 \mathrm{~kW}$ & $\rho=1.225 \mathrm{~kg} / \mathrm{m}^{3}$ & $r=6.25 \mathrm{~m}$ & $v_{w_{c u t-i n}}=5 \mathrm{~m} / \mathrm{s}$ \\
\hline$v_{w_{\text {rated }}}=12 \mathrm{~m} / \mathrm{s}$ & $v_{w_{\text {cut }- \text { out }}}=20 \mathrm{~m} / \mathrm{s}$ & $\omega_{m}=14.5 \mathrm{rpm}$ & $\beta=0^{\circ}$ \\
\hline$\lambda_{\text {opt }}=8.1$ & $C_{p_{\max }}=0.48$ & $J_{m}=0.23 \mathrm{~kg} \cdot \mathrm{m}^{2}$ & $\begin{array}{l}\text { Gear box }: 3 \text { stages } \\
\text { Ratio }: 5: 1 \text { per stage } \\
\quad\left(n_{\text {gear }}=125\right)\end{array}$ \\
\hline $\begin{array}{r}K_{s e}=4.4 x \\
(0.3 p u\end{array}$ & $\begin{array}{l}0^{3} \quad N \cdot \mathrm{m} / \mathrm{rad} \\
\left.\text { ff } T_{m} / \delta \theta\right)\end{array}$ & $\begin{array}{r}D_{s e}=1.6 \times 1 \\
\quad(1 \mathrm{pu} \text { of } T\end{array}$ & $\begin{array}{l}{ }^{3} N \cdot \mathrm{m} / \mathrm{rad} / \mathrm{s} \\
\left.{ }^{2} / \mathrm{pu} \delta \omega\right)\end{array}$ \\
\hline \multicolumn{4}{|c|}{4 pole-Self-excited induction generator } \\
\hline$P_{g}=20 \mathrm{~kW}$ & $\begin{array}{l}V_{\text {stator }} \\
=460 V_{L L r m s}\end{array}$ & $f=60 \mathrm{~Hz}$ & $\omega_{g}=1812 \mathrm{rpm}$ \\
\hline$T_{e}=105 \mathrm{~N} . \mathrm{m}$ & $p f_{g}=0.86$ & $J_{g}=0.07 \mathrm{~kg} \cdot \mathrm{m}^{2}$ & $I_{\text {stator }}=29 A_{\text {rms }}$ \\
\hline$R_{S}=0.1325 \Omega$ & $L_{l s}=3.7 \mathrm{mH}$ & $R_{r}^{\prime}=0.1242 \Omega$ & $L_{l r}^{\prime}=3.7 \mathrm{mH}$ \\
\hline$L_{m}=63.69 \mathrm{mH}$ & $F=0.0576 \mathrm{~N} . \mathrm{m} . \mathrm{s}$ & $\begin{array}{c}C_{g}=140 \mu F / \text { phase } \\
(0.48 \mathrm{pu})\end{array}$ & $\begin{array}{c}C_{d c}=100 \mu F \\
(0.34 \mathrm{pu})\end{array}$ \\
\hline \multicolumn{4}{|c|}{$\begin{array}{l}\text { Lead acid battery bank ( } 408 \mathrm{~V} 300 \mathrm{Ah}) \text { at } 100 \% \mathrm{SoC} \\
\text { (Obtained by combining } 34 \text { units in series, each } 12 \mathrm{~V} 300 \mathrm{Ah}, E_{o}=12.4 \mathrm{~V}, K_{E}=1.1 \mathrm{~V}, R_{o}= \\
\left.1 \mathrm{~m} \Omega \text { at } 25^{\circ} \mathrm{C}\right) \text {. }\end{array}$} \\
\hline$A_{o}=5 \mathrm{~m} \Omega$ & $D_{L}=60 \mathrm{kWh}$ & $u=1$ & $k_{T}=1$ \\
\hline$R_{L}=50 \mathrm{~m} \Omega$ & $R_{c}=10 \mathrm{~m} \Omega$ & $L_{b}=6.6 \mu \mathrm{H}$ & $C_{b}=3.7 \mathrm{mF}$ \\
\hline \multicolumn{4}{|c|}{ DC-Link and output C-filter } \\
\hline$L_{d c}=16.2$ & $m H(0.67 \mathrm{pu})$ & $\begin{array}{l}i_{d c}=55.5 \mathrm{~A} \\
f_{s}=5.1 \mathrm{kHz}\end{array}$ & $\begin{aligned} C_{i}= & 125 \mu F / \text { phase } \\
& (0.42 \mathrm{pu})\end{aligned}$ \\
\hline \multicolumn{4}{|c|}{ Extended Generic Load Model (rated load) } \\
\hline$V_{o}=310.3 \mathrm{~V}$ & $\omega_{L_{o}}=377 \mathrm{rad} / \mathrm{s}$ & $\alpha_{p}=\alpha_{q}=2$ & $\beta_{p}=\beta_{q}=0$ \\
\hline $\begin{array}{c}P_{o}^{+}=20 \mathrm{~kW} \\
Y_{P}^{+}=0.1385 \mathrm{~W} / \mathrm{V}^{2}\end{array}$ & $\begin{array}{c}Q_{o}^{+}=10 \mathrm{kVar} \\
Y_{Q}^{+}=0.0692 \mathrm{Var} / \mathrm{V}^{2}\end{array}$ & $\begin{array}{c}Q_{p d o}^{-}=-5.08 \mathrm{kVar} \\
Y_{Q_{p d}^{-}}^{-}=-0.0352 \mathrm{Var} / \mathrm{V}^{2}\end{array}$ & $\begin{array}{c}Q_{p q o}^{-}=-6.04 \mathrm{kVar} \\
Y_{Q_{p q}}^{-}=-0.0418 \mathrm{Var} / \mathrm{V}^{2}\end{array}$ \\
\hline
\end{tabular}




\section{Appendix B}

\section{Design of Battery-Side L-C filter}

Resonant frequency and damping ratio of the L-C filter are given by (B.1) and (B.2), respectively, where $R_{L}$ and $R_{C}$ represent the equivalent series resistances (ESRs) of filter inductor and capacitor, respectively.

$$
\begin{gathered}
f_{r}=\frac{1}{2 \pi \sqrt{L_{b} C_{b}}} \\
\xi=\frac{R_{L}+R_{c}}{2} \sqrt{\frac{C_{b}}{L_{b}}}
\end{gathered}
$$

The frequency of the dominant unwanted component to be suppressed is the ripple frequency of the current on the battery-side of the reduced H-bridge, which is the same as the switching frequency $\left(f_{s}\right)$. Typically, $f_{r}$ is selected to be one decade below $f_{s}\left(f_{r}=0.1 f_{s}\right)$ and the damping ratio is set equal to $1 / \sqrt{2}$. Solving (B.1) and (B.2) yields $L_{b}$ and $C_{b}$. It should be noticed that the ripple frequency of the current on the battery-side of the reduced $\mathrm{H}$-bridge converter is effectively doubled under PWM unipolar voltage switching scheme.

Based on (B.1) and (B.2) and the parameters of the 20kW WECS given in Table A.2 (i.e., $R_{L}=$ $\left.50 \mathrm{~m} \Omega, R_{c}=10 \mathrm{~m} \Omega, f_{s}=5.1 \mathrm{kHz}\right)$ the battery-side LC filter parameters are obtained as $L_{b}=6.6 \mu \mathrm{H}$ and $C_{b}=3.7 \mathrm{mF}$. 


\section{Appendix $C$}

\section{Switching States of Current Source Inverter}

The schematic diagram of a three-phase CSI, feeding a three-phase load, is shown in Fig. C.1.

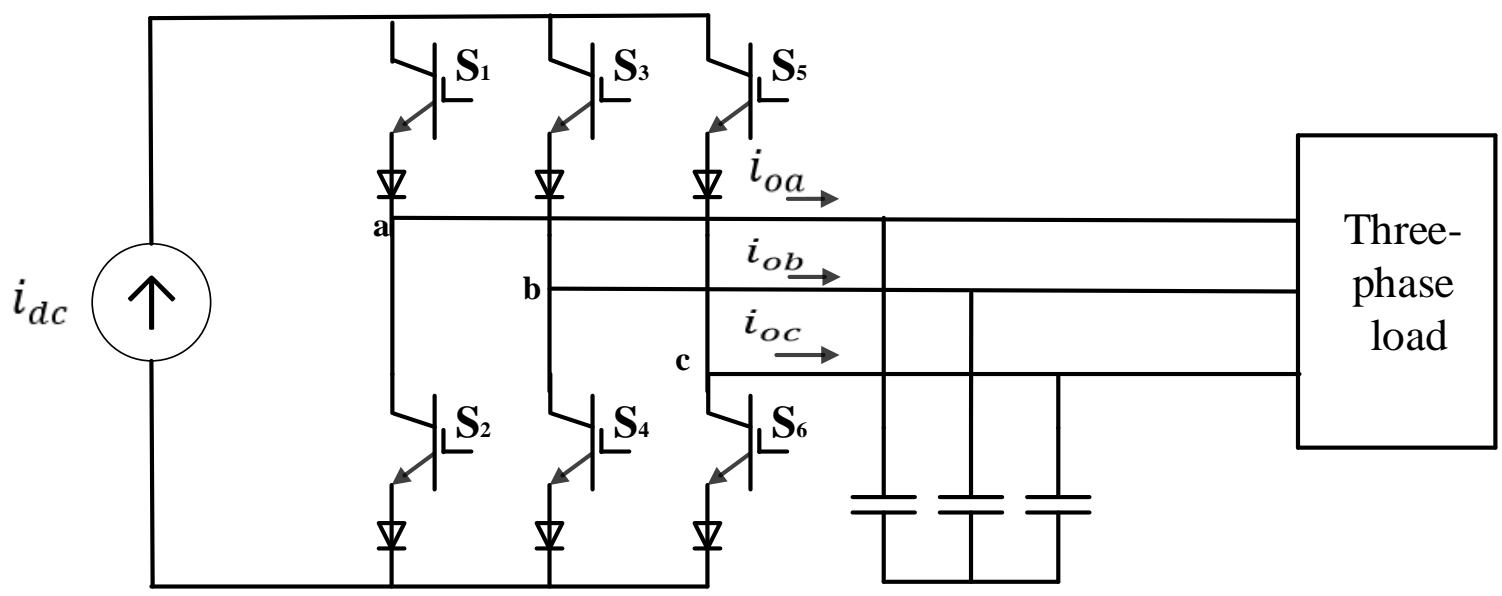

C-Filter

Fig. C.1: Three phase CSI feeding three-phase load.

In order to satisfy KVL and KCL in three-phase CSI, two constraints for CSI switching must be always met. The dc link of CSI acts as a current source and cannot be open-circuited. Thus, at least one top switch and one bottom switch must be on at any instant of time. On the other hand, in order to produce defined three-phase current waveforms at the ac side, at most one top switch and one bottom switch must be on at any instant of time. In other words, one and only one top switch and one and only one bottom switch must be on at any instant of time. This statement applies to current source rectifier as well. In practice, the switching of devices in a converter is not instantaneous. Therefore, an overlap between the on-periods of outgoing and incoming switches is necessary to prevent interruption of the dc-link current. The filter capacitors on the ac side will facilitate commutation of switches, besides filtering the switching harmonics.

Based on the constraints mentioned above, there are nine valid switching states in the operation of CSI, as shown in Table C.1. States 1 to 6 are active states, where current flows from dc side to the load. On the other hand, states 7 to 9 are zero states, where the dc link current $\left(i_{d c}\right)$ freewheels 
through one of the inverter legs. Thus, no power transfers from dc side to load during these states. They are also known as shoot-through states. The inverter must change state according to a certain pattern in order to generate a set of ac line current. The resulting ac currents consists of discrete values, that are $i_{d c}, 0$ or $-i_{d c}$, as noticed in Table C.1.

Table C.1: Switching States for a Three-Phase CSI.

\begin{tabular}{|c|c|c|c|c|c|}
\hline & State no. & ON Switches & $i_{o a}$ & $i_{o b}$ & $i_{o c}$ \\
\hline \multirow{6}{*}{$\begin{array}{l}\text { Active } \\
\text { States }\end{array}$} & 1 & $S_{1} \& S_{4}$ & $i_{d c}$ & $-i_{d c}$ & 0 \\
\hline & 2 & $S_{1} \& S_{6}$ & $i_{d c}$ & 0 & $-i_{d c}$ \\
\hline & 3 & $S_{3} \& S_{2}$ & $-i_{d c}$ & $i_{d c}$ & 0 \\
\hline & 4 & $S_{3} \& S_{6}$ & 0 & $i_{d c}$ & $-i_{d c}$ \\
\hline & 5 & $S_{5} \& S_{2}$ & $-i_{d c}$ & 0 & $i_{d c}$ \\
\hline & 6 & $S_{5} \& S_{4}$ & 0 & $-i_{d c}$ & $i_{d c}$ \\
\hline \multirow[t]{3}{*}{ Zero States } & 7 & $S_{1} \& S_{2}$ & 0 & 0 & 0 \\
\hline & 8 & $S_{3} \& S_{4}$ & 0 & 0 & 0 \\
\hline & 9 & $S_{5} \& S_{6}$ & 0 & 0 & 0 \\
\hline
\end{tabular}




\section{Appendix D}

\section{Overall Dynamic Model Equations of the Proposed WECS}

The overall dynamic model of the proposed wind turbine system contains equations of the mechanical drive train, self-excited induction generator, battery-side LC filter, current-source inverter supplying the extended generic load model, and dc-link inductor. The equations for each component, as well as the overall system, are derived in the following sections.

\section{D.1 Equations of the 2-Mass Drive Train}

From equations (4.13)-(4.15), one gets:

$$
\begin{gathered}
\frac{d}{d t} \omega_{m}=\frac{1}{J_{m}}\left[T_{m}-D_{s e} \delta \omega-K_{s e} \delta \theta\right] \\
\frac{d}{d t} \omega_{g}=\frac{1}{J_{g}}\left[\frac{1}{n_{\text {gear }}} D_{\text {se }} \delta \omega+\frac{1}{n_{\text {gear }}} K_{\text {se }} \delta \theta-T_{e}\right] \\
\frac{d(\delta \theta)}{d t}=\frac{d}{d t}\left(\theta_{m}-\frac{\theta_{g}}{n_{\text {gear }}}\right)=\delta \omega=\omega_{m}-\frac{\omega_{g}}{n_{\text {gear }}}
\end{gathered}
$$

where $T_{m}, T_{e}$, and $C_{p}$, given in (D.4)-(D.6), are the turbine mechanical torque, the electromagnetic torque of the induction generator, and the turbine's performance coefficient, respectively.

$$
\begin{gathered}
T_{m}=\frac{\rho \pi r^{2}}{2 \omega_{m}} v_{w}^{3} C_{p}(\lambda, \beta) \\
T_{e}=\frac{3 P L_{m}}{2}\left(i_{q s} i_{d r}^{\prime}-i_{d s} i_{q r}^{\prime}\right) \\
C_{p}(\lambda, \beta)=0.5176\left(\frac{116}{\lambda_{i}}-0.4 \beta-5\right) e^{-\frac{21}{\lambda_{i}}}+0.0068 \lambda
\end{gathered}
$$

Equations (D.7) and (D.8) give relations for $\lambda_{i}$ and $\lambda$.

$$
\begin{gathered}
\frac{1}{\lambda_{i}}=\frac{1}{\lambda+0.08 \beta}-\frac{0.035}{\beta^{3}+1} \\
\lambda=\frac{\omega_{m} r}{v_{w}}
\end{gathered}
$$

For the wind turbine system under study, it is assumed that the rotor pitch angle is fixed at zero (i.e., $\beta=0$ ). Hence, equation (D.7) becomes 


$$
\frac{1}{\lambda_{i}}=\frac{1}{\lambda}-0.035
$$

Substituting (D.9) in (D.6) yields

$$
C_{p}(\lambda, 0)=\left(\frac{60}{\lambda}-4.69\right) e^{\left(-\frac{21}{\lambda}+0.74\right)}+0.0068 \lambda
$$

Substituting $\lambda$ from (D.8) in (D.10) yields

$$
C_{p}(\lambda, 0)=\left(\frac{60 v_{w}}{\omega_{m} r}-4.69\right) e^{\left(-\frac{21 v_{w}}{\omega_{m} r}+0.74\right)}+0.0068 \frac{\omega_{m} r}{v_{w}}
$$

Substituting $C_{p}$ from (D.11) in (D.4) gives

$$
T_{m}=\frac{\rho \pi r^{2}}{2 \omega_{m}} v_{w}^{3}\left[\left(\frac{60 v_{w}}{\omega_{m} r}-4.69\right) e^{\left(-\frac{21 v_{w}}{\omega_{m} r}+0.74\right)}+0.0068 \frac{\omega_{m} r}{v_{w}}\right]
$$

Substituting (D.12) and (D.5) in (D.1) and (D.2), respectively, yields

$$
\begin{gathered}
\frac{d}{d t} \omega_{m}=\frac{1}{J_{m}}\left[\rho \pi r^{2}\left(\left(\frac{30 v_{w}^{3}}{\omega_{m}^{2} r}-\frac{2.35 v_{w}^{2}}{\omega_{m}}\right) e^{\left(-\frac{21 v_{w}}{\omega_{m} r}+0.74\right)}+0.0034 r v_{w}\right) v_{w}-K_{s e} \delta \theta-\right. \\
\left.D_{s e}\left(\omega_{m}-\frac{\omega_{r}}{P n_{\text {gear }}}\right)\right] \\
\frac{d}{d t} \omega_{r}=\frac{P}{J_{g}}\left[\frac{1}{n_{\text {gear }}} K_{s e} \delta \theta+\frac{1}{n_{\text {gear }}} D_{s e}\left(\omega_{m}-\frac{\omega_{r}}{P n_{\text {gear }}}\right)-\frac{3 P L_{m}}{2}\left(i_{q s} i_{d r}^{\prime}-i_{d s} i_{q r}^{\prime}\right)\right]
\end{gathered}
$$

The mechanical angular speed of the induction generator shaft $\left(\omega_{g}\right)$ is related to the electrical angular speed $\left(\omega_{r}\right)$ through the pole pair $(P)$ of the machine, i.e., $\omega_{g}=\omega_{r} / P$. Thus, from (D.3),

$$
\frac{d}{d t}(\delta \theta)=\omega_{m}-\frac{\omega_{r}}{P n_{g e a r}}
$$

\section{D.2 Equations of Self-Excited Induction Generator with Diode Bridge Rectifier}

From $d q$ state space matrix equation given in (4.26), the $d q$ stator and rotor currents of the selfexcited induction generator are

$$
\begin{gathered}
\frac{d}{d t} i_{q s}=\frac{1}{k_{s}}\left[R_{s} L_{r}^{\prime} i_{q s}+\left(\omega_{e}\left(L_{s} L_{r}^{\prime}-L_{m}^{2}\right)+\omega_{r} L_{m}^{2}\right) i_{d s}-R_{r}^{\prime} L_{m} i_{q r}^{\prime}+\right. \\
\left.\omega_{r} L_{m} L_{r}^{\prime} i_{d r}^{\prime}-L_{r}^{\prime} v_{c_{g q}}\right] \\
\frac{d}{d t} i_{d s}=\frac{1}{k_{s}}\left[\left(\omega_{e}\left(L_{m}^{2}-L_{s} L_{r}^{\prime}\right)-\omega_{r} L_{m}^{2}\right) i_{q s}+R_{s} L_{r}^{\prime} i_{d s}-\omega_{r} L_{m} L_{r}^{\prime} i_{q r}^{\prime}-R_{r}^{\prime} L_{m} i_{d r}^{\prime}-L_{r}^{\prime} v_{c_{g d}}\right]
\end{gathered}
$$




$$
\begin{gathered}
\frac{d}{d t} i_{q r}^{\prime}=\frac{1}{k_{s}}\left[-R_{s} L_{m} i_{q s}-\omega_{r} L_{m} L_{s} i_{d s}+R_{r}^{\prime} L_{s} i_{q r}^{\prime}+\left(\omega_{e}\left(L_{s} L_{r}^{\prime}-L_{m}^{2}\right)-L_{s} L_{r}^{\prime} \omega_{r}\right) i_{d r}^{\prime}+\right. \\
\left.L_{m} v_{c_{g q}}\right] \\
\frac{d}{d t} i_{d r}^{\prime}=\frac{1}{k_{s}}\left[\omega_{r} L_{m} L_{s} i_{q s}-R_{s} L_{m} i_{d s}+\left(\omega_{e}\left(L_{m}^{2}-L_{s} L_{r}^{\prime}\right)+L_{s} L_{r}^{\prime} \omega_{r}\right) i_{q r}^{\prime}+R_{r}^{\prime} L_{s} i_{d r}^{\prime}+\right. \\
\left.L_{m} v_{c_{g d}}\right]
\end{gathered}
$$

where $k_{s}=L_{m}^{2}-L_{s} L_{r}^{\prime}$.

From (4.33) and (4.34), the dynamics of the excitation capacitor voltage is described by

$$
\begin{gathered}
\frac{d}{d t} v_{c_{g d}}=-\frac{1}{c_{g}}\left(i_{d s}+\frac{2 \sqrt{3}}{\pi} d_{b} i_{d c}\right)+\omega_{e} v_{c_{g q}} \\
\frac{d}{d t} v_{c_{g q}}=-\frac{1}{c_{g}} i_{q s}-\omega_{e} v_{c_{g d}}
\end{gathered}
$$

\section{D.3 Battery-Side LC Filter Equations}

From (4.46) and (4.47), the dynamic of battery-side LC filter is described by

$$
\begin{gathered}
\frac{d}{d t} i_{b a t}=\frac{1}{L_{b}}\left(E_{o}-K_{E}(1-S o C)-R_{o}\left(1+A_{0}(1-S o C)\right) i_{b a t}-v_{c_{b}}\right) \\
\frac{d}{d t} v_{c_{b}}=\frac{1}{c_{b}}\left(i_{b a t}-\left(2 d_{A}-1\right) i_{d c}\right)
\end{gathered}
$$

\section{D.4 Equations of Current Source Inverter Supplying the Extended Generic Load Model}

From $d q$ state space matrix equation given in (4.62), the dynamic of the CSI capacitive filter is described by

$$
\begin{aligned}
& \frac{d}{d t} v_{c i d}^{+}=\frac{1}{C_{i}} i_{o d}^{+}+\omega_{L} v_{c i q}^{+}-\frac{1}{C_{i}} i_{p d}^{+} \\
& \frac{d}{d t} v_{c i q}^{+}=\frac{1}{C_{i}} i_{o q}^{+}-\omega_{L} v_{c i d}^{+}-\frac{1}{C_{i}} i_{p q}^{+} \\
& \frac{d}{d t} v_{c i d}^{-}=\frac{1}{C_{i}} i_{o d}^{-}-\omega_{L} v_{c i q}^{-}-\frac{1}{C_{i}} i_{p d}^{-} \\
& \frac{d}{d t} v_{c i q}^{-}=\frac{1}{C_{i}} i_{o q}^{-}+\omega_{L} v_{c i d}^{-}-\frac{1}{C_{i}} i_{p q}^{-}
\end{aligned}
$$

From (4.60), the fundamental $d q$-axis output currents of CSI are: 


$$
\begin{aligned}
& i_{o d}^{+}=G m_{i d}^{+} i_{d c} \\
& i_{o q}^{+}=G m_{i q}^{+} i_{d c} \\
& i_{o d}^{-}=G m_{i d}^{-} i_{d c} \\
& i_{o q}^{-}=G m_{i q}^{-} i_{d c}
\end{aligned}
$$

From the $d q$ state space matrix of the extended generic load model given in Table 4.4, the dynamics of the load model is described by the following equations.

$$
\begin{gathered}
\frac{d}{d t} x_{1 m}^{+}=-\left(d^{2}+\omega_{o}^{2}\right) x_{2 m}^{+}+v_{c i d}^{+} \\
\frac{d}{d t} x_{2 m}^{+}=x_{1 m}^{+}+2 d x_{2 m}^{+} \\
\frac{d}{d t} x_{1 m}^{-}=-\left(d^{2}+\omega_{o}^{2}\right) x_{2 m}^{-}+v_{c i d}^{+} \\
\frac{d}{d t} x_{2 m}^{-}=x_{1 m}^{-}+2 d x_{2 m}^{-}
\end{gathered}
$$

From the same table (Table 4.4), the $d q$ outputs currents of the extended generic load model are

$$
\begin{gathered}
i_{p d}^{+}=Y_{P}^{+}\left(d^{2}+\omega_{o}^{2}\right) x_{2 m}^{+} \\
i_{p q}^{+}=-Y_{Q}^{+}\left(d^{2}+\omega_{o}^{2}\right) x_{2 m}^{+} \\
i_{p d}^{-}=Y_{Q_{p d}}^{-}\left(d^{2}+\omega_{o}^{2}\right) x_{2 m}^{-} \\
i_{p q}^{-}=-Y_{Q_{p q}}^{-}\left(d^{2}+\omega_{o}^{2}\right) x_{2 m}^{-}
\end{gathered}
$$

Substituting (D.28)-(D.31) and (D.36)-(D.39) in (D.24)-(D.27), yields

$$
\begin{aligned}
& \frac{d}{d t} v_{c i d}^{+}=\frac{1}{C_{i}}\left(G m_{i d}^{+} i_{d c}-Y_{P}^{+}\left(d^{2}+\omega_{o}^{2}\right) x_{2 m}^{+}\right)+\omega_{L} v_{c i q}^{+} \\
& \frac{d}{d t} v_{c i q}^{+}=\frac{1}{C_{i}}\left(G m_{i q}^{+} i_{d c}+Y_{Q}^{+}\left(d^{2}+\omega_{o}^{2}\right) x_{2 m}^{+}\right)-\omega_{L} v_{c i d}^{+} \\
& \frac{d}{d t} v_{c i d}^{-}=\frac{1}{C_{i}}\left(G m_{i d}^{-} i_{d c}-Y_{Q_{p d}}^{-}\left(d^{2}+\omega_{o}^{2}\right) x_{2 m}^{-}\right)-\omega_{L} v_{c i q}^{-} \\
& \frac{d}{d t} v_{c i q}^{-}=\frac{1}{C_{i}}\left(G m_{i q}^{-} i_{d c}+Y_{Q_{p q}}^{-}\left(d^{2}+\omega_{o}^{2}\right) x_{2 m}^{-}\right)+\omega_{L} v_{c i d}^{-}
\end{aligned}
$$




\section{D.5 DC-Link Equations}

From (4.82), the dynamics of the dc-link current is described by

$$
\frac{d}{d t} i_{d c}=\frac{1}{L_{d c}}\left(v_{d}+v_{x y}-v_{i n v}\right)
$$

where $v_{d}, v_{x y}$, and $v_{i n v}$ were defined in (4.36), (4.44), and (4.61), respectively, as

$$
\begin{gathered}
v_{d}=d_{b} v_{d c} \\
v_{x y}=\left(2 d_{A}-1\right) v_{c_{b}} \\
v_{i n v}=1.5 G\left(m_{i d}^{+} v_{c i d}^{+}+m_{i q}^{+} v_{c i q}^{+}+m_{i d}^{-} v_{c i d}^{-}+m_{i q}^{-} v_{c i q}^{-}\right)
\end{gathered}
$$

In (D.45), $v_{d c}=3 \sqrt{3}\left(v_{c_{g d}}\right) / \pi$.

Substituting (D.45)-(D.47) in (D.44), yields

$$
\begin{gathered}
\frac{d}{d t} i_{d c}=\frac{1}{L_{d c}} \frac{3 \sqrt{3}}{\pi} d_{b} v_{c g d}+\frac{1}{L_{d c}}\left(2 d_{A}-1\right) v_{c_{b}}-\frac{1.5}{L_{d c}} G\left(m_{i d}^{+} v_{c i d}^{+}+m_{i q}^{+} v_{c i q}^{+}+\right. \\
\left.m_{\text {id }}^{-} v_{\text {cid }}^{-}+m_{\text {iq }}^{-} v_{c i q}^{-}\right)
\end{gathered}
$$

\section{D.6 Complete Dynamic Equations}

By combining equations (D.13)-(D.23), (D.32)-(D.35), (D.40)-(D.43), and (D.48), the complete dynamic model of the proposed WECS can be summarized in the form of 20 state equations, as given in (4.84). 


\section{Appendix $E$}

\section{Small-Signal Models for CSI-SCIG-WECS}

The following sections give the details of the small-signal models of the Wind-Turbine Generation (WTG) subsystem, Energy Storage (ES) subsystem, Current Source Inverter-Load (CSI-Load) subsystem, and the entire system.

In small-signal model matrices, capital letter of a variable (input or state variable) indicates the steady-state value of the variable at the selected operating point for linearization.

\section{E.1 WTG Subsystem}

1) State-Space Equations

where

$$
\frac{d}{d t} X_{g}=A_{g} X_{g}+B_{g} U_{g}
$$

$$
\begin{aligned}
& X_{g}=\left[\begin{array}{lllllllll}
\omega_{m} & \omega_{r} & \delta \theta & i_{q s} & i_{d s} & i_{q r}^{\prime} & i_{d r}^{\prime} & v_{c g q} & v_{c g d}
\end{array}\right]^{T} \\
& U_{g}=\left[\begin{array}{lll}
v_{w} & d_{b} & i_{d c}
\end{array}\right]^{T} \\
& A_{g}=\left[\begin{array}{ccccccccc}
-\frac{D_{s e}}{J_{m}} & \frac{D_{s e}}{J_{m} P n_{\text {gear }}} & -\frac{K_{s e}}{J_{m}} & 0 & 0 & 0 & 0 & 0 & 0 \\
\frac{P D_{s e}}{J_{g} n_{\text {gear }}} & -\frac{D_{s e}}{J_{g} n_{\text {gear }}^{2}} & \frac{P K_{s e}}{J_{g} n_{\text {gear }}} & -\frac{3 P^{2} L_{m}}{4 J_{g}} i_{d r}^{\prime} & \frac{3 P^{2} L_{m}}{4 J_{g}} i_{q r}^{\prime} & \frac{3 P^{2} L_{m}}{4 J_{g}} i_{d s} & -\frac{3 P^{2} L_{m}}{4 J_{g}} i_{q s} & 0 & 0 \\
1 & -\frac{1}{P n_{\text {gear }}} & 0 & 0 & 0 & 0 & 0 & 0 & 0 \\
0 & 0 & 0 & \frac{R_{s} L_{r}^{\prime}}{k_{s}} & a_{45} & -\frac{R_{r}^{\prime} L_{m}}{k_{s}} & \frac{\omega_{r} L_{m} L_{r}^{\prime}}{k_{s}} & -\frac{L_{r}^{\prime}}{k_{s}} & 0 \\
0 & 0 & 0 & -a_{45} & \frac{R_{s} L_{r}^{\prime}}{k_{s}} & -\frac{\omega_{r} L_{m} L_{r}^{\prime}}{k_{s}} & -\frac{R_{r}^{\prime} L_{m}}{k_{s}} & 0 & -\frac{L_{r}^{\prime}}{k_{s}} \\
0 & 0 & 0 & -\frac{R_{s} L_{m}}{k_{s}} & -\frac{\omega_{r} L_{m} L_{s}}{k_{s}} & \frac{R_{r}^{\prime} L_{s}}{k_{s}} & a_{67} & \frac{L_{m}}{k_{s}} & 0 \\
0 & 0 & 0 & \frac{\omega_{r} L_{m} L_{s}}{k_{s}} & -\frac{R_{s} L_{m}}{k_{s}} & -a_{67} & \frac{R_{r}^{\prime} L_{s}}{k_{s}} & 0 & \frac{L_{m}}{k_{s}} \\
0 & 0 & 0 & -\frac{1}{C_{g}} & 0 & 0 & 0 & 0 & -\omega_{e} \\
0 & 0 & 0 & 0 & -\frac{1}{C_{g}} & 0 & 0 & \omega_{e} & 0
\end{array}\right]
\end{aligned}
$$




$$
B_{g}=\left[\begin{array}{ccc}
b_{11} & 0 & 0 \\
0 & 0 & 0 \\
0 & 0 & 0 \\
0 & 0 & 0 \\
0 & 0 & 0 \\
0 & 0 & 0 \\
0 & 0 & 0 \\
0 & 0 & 0 \\
0 & 0 & -\frac{2 \sqrt{3}}{\pi C_{g}} d_{b}
\end{array}\right]
$$

In (E.4) and (E.5),

$$
\begin{aligned}
& a_{45}=\frac{1}{k_{s}}\left(\omega_{e}\left(L_{s} L_{r}^{\prime}-L_{m}^{2}\right)+\omega_{r} L_{m}^{2}\right), a_{67}=\frac{1}{k_{s}}\left(\omega_{e}\left(L_{s} L_{r}^{\prime}-L_{m}^{2}\right)-L_{s} L_{r}^{\prime} \omega_{r}\right), \omega_{e}=\frac{R_{s} i_{d s}-v_{c g d}}{L_{s} i_{q s}+L_{m} i_{q r}^{\prime}}, \\
& b_{11}=\frac{\rho \pi r^{2}}{J_{m}}\left[\left(\frac{30 v_{\omega}^{3}}{\omega_{m}^{2} r}-\frac{2.35 v_{\omega}^{2}}{\omega_{m}}\right) e^{\left(-\frac{21 v_{w}}{\omega_{m} r}+0.74\right)}+0.0034 r v_{\omega}\right] .
\end{aligned}
$$

\section{2) Small-Signal Equations}

$$
\frac{d}{d t} \Delta X_{g}=A_{g}^{\prime} \Delta X_{g}+B_{g}^{\prime} \Delta U_{g}
$$

where

$$
\begin{aligned}
& \Delta X_{g}=\left[\begin{array}{lllllllll}
\Delta \omega_{m} & \Delta \omega_{r} & \Delta \delta \theta & \Delta i_{q s} & \Delta i_{d s} & \Delta i_{q r}^{\prime} & \Delta i_{d r}^{\prime} & \Delta v_{c g q} & \Delta v_{c g d}
\end{array}\right]^{T} \\
& \Delta U_{g}=\left[\begin{array}{lll}
\Delta v_{w} & \Delta d_{b} \Delta i_{d c}
\end{array}\right]^{T} \\
& A_{g}^{\prime}=\left[\begin{array}{ccccccccc}
a_{11}^{\prime} & \frac{D_{s e}}{J_{m} P n_{\text {gear }}} & -\frac{K_{s e}}{J_{m}} & 0 & 0 & 0 & 0 & 0 & 0 \\
\frac{P D_{s e}}{J_{g} n_{\text {gear }}} & -\frac{D_{s e}}{J_{g} n_{\text {gear }}^{2}} & \frac{P K_{s e}}{J_{g} n_{\text {gear }}} & -\frac{3 P^{2} L_{m}}{2 J_{g}} I_{d r}^{\prime} & \frac{3 P^{2} L_{m}}{2 J_{g}} I_{q r}^{\prime} & \frac{3 P^{2} L_{m}}{2 J_{g}} I_{d s} & -\frac{3 P^{2} L_{m}}{2 J_{g}} I_{q s} & 0 & 0 \\
1 & -\frac{1}{P n_{\text {gear }}} & 0 & 0 & 0 & 0 & 0 & 0 & 0 \\
0 & \frac{L_{m}^{2}}{k_{s}} I_{d s}+\frac{L_{m} L_{r}^{\prime}}{k_{s}} I_{d r}^{\prime} & 0 & a_{44}^{\prime} & a_{45}^{\prime} & a_{46}^{\prime} & \frac{W_{r} L_{m} L_{r}^{\prime}}{k_{s}} & -\frac{L_{r}^{\prime}}{k_{s}} & a_{49}^{\prime} \\
0 & -\frac{L_{m}^{2}}{k_{s}} I_{q s}-\frac{L_{m} L_{r}^{\prime}}{k_{s}} I_{q r}^{\prime} & 0 & a_{54}^{\prime} & a_{55}^{\prime} & a_{56}^{\prime} & -\frac{R_{r}^{\prime} L_{m}}{k_{s}} & 0 & a_{59}^{\prime} \\
0 & -\frac{L_{m} L_{s}}{k_{s}} I_{d s}-\frac{L_{s} L_{r}^{\prime}}{k_{s}} I_{d r}^{\prime} & 0 & a_{64}^{\prime} & a_{65}^{\prime} & a_{66}^{\prime} & a_{67}^{\prime} & \frac{L_{m}}{k_{s}} & a_{69}^{\prime} \\
0 & \frac{L_{m} L_{s}}{k_{s}} I_{q s}+\frac{L_{s} L_{r}^{\prime}}{k_{s}} I_{q r}^{\prime} & 0 & a_{74}^{\prime} & a_{75}^{\prime} & a_{76}^{\prime} & \frac{R_{r}^{\prime} L_{s}}{k_{s}} & 0 & a_{79}^{\prime} \\
0 & 0 & 0 & a_{84}^{\prime} & a_{85}^{\prime} & a_{86}^{\prime} & 0 & 0 & a_{89}^{\prime} \\
0 & 0 & 0 & a_{94}^{\prime} & a_{95}^{\prime} & a_{96}^{\prime} & 0 & W_{e} & a_{99}^{\prime}
\end{array}\right]
\end{aligned}
$$




$$
B_{g}^{\prime}=\left[\begin{array}{ccc}
b_{11}^{\prime} & 0 & 0 \\
0 & 0 & 0 \\
0 & 0 & 0 \\
0 & 0 & 0 \\
0 & 0 & 0 \\
0 & 0 & 0 \\
0 & 0 & 0 \\
0 & 0 & 0 \\
0 & -\frac{2 \sqrt{3}}{\pi C_{g}} I_{d c} & -\frac{2 \sqrt{3}}{\pi C_{g}} D_{b}
\end{array}\right]
$$

In (E.9) and (E.10),

$$
\begin{aligned}
& a_{11}^{\prime}=\frac{1}{J_{m}}\left[-D_{s e}+\rho \pi r^{2}\left(-\frac{60 V_{\omega}^{4}}{W_{m}^{3} r}+\frac{2.35 V_{\omega}^{3}}{W_{m}^{2}}\right) e^{\left(-\frac{21 V_{w}}{W_{m} r}+0.74\right)}+\frac{1}{w_{m}^{2}}\left(2 1 \rho \pi r V _ { \omega } \left(\frac{30 V_{\omega}^{4}}{W_{m}^{2} r}-\right.\right.\right. \\
& \left.\left.\left.\frac{2.35 V_{\omega}^{3}}{W_{m}}\right) e^{\left(-\frac{21 V_{w}}{W_{m} r}+0.74\right)}\right)\right], W_{e}=\frac{R_{S} I_{d s}-V_{c g d}}{L_{s} I_{q s}+L_{m} I_{q r}^{\prime}}, a_{44}^{\prime}=\frac{1}{k_{s}}\left[R_{s} L_{r}^{\prime}-\frac{\left(R_{s} I_{d s}-V_{c g d}\right)\left(L_{s} L_{r}^{\prime}-L_{m}^{2}\right) L_{s} I_{d s}}{\left(L_{s} I_{q s}+L_{m} I_{q r}^{\prime}\right)^{2}}\right], \\
& a_{45}^{\prime}=\frac{1}{k_{s}}\left[\frac{R_{s} I_{d s}\left(L_{s} L_{r}^{\prime}-L_{m}^{2}\right)+\left(R_{S} I_{d s}-V_{c g d}\right)\left(L_{s} L_{r}^{\prime}-L_{m}^{2}\right)}{\left(L_{s} I_{q S}+L_{m} I_{q r}^{\prime}\right)}+W_{r} L_{m}^{2}\right], \\
& a_{46}^{\prime}=\frac{1}{k_{s}}\left[-R_{r}^{\prime} L_{m}-\frac{\left(R_{s} I_{d s}-V_{c g d}\right)\left(L_{s} L_{r}^{\prime}-L_{m}^{2}\right) L_{m} I_{d s}}{\left(L_{s} I_{q s}+L_{m} I_{q r}^{\prime}\right)^{2}}\right], a_{49}^{\prime}=-\frac{\left(L_{s} L_{r}^{\prime}-L_{m}^{2}\right) I_{d s}}{k_{s}\left(L_{s} I_{q s}+L_{m} I_{q r}^{\prime}\right)^{\prime}}, \\
& a_{54}^{\prime}=\frac{1}{k_{s}}\left[-\frac{\left(R_{s} I_{d s}-V_{c g d}\right)\left(L_{m}^{2}-L_{s} L_{r}^{\prime}\right) L_{s} I_{q s}}{\left(L_{s} I_{q s}+L_{m} I_{q r}^{\prime}\right)^{2}}+\frac{\left(R_{s} I_{d s}-V_{c g d}\right)\left(L_{m}^{2}-L_{s} L_{r}^{\prime}\right)}{\left(L_{s} I_{q s}+L_{m} I_{q r}^{\prime}\right)}-W_{r} L_{m}^{2}\right], \\
& a_{55}^{\prime}=\frac{1}{k_{s}}\left[\frac{R_{s} I_{q s}\left(L_{m}^{2}-L_{s} L_{r}^{\prime}\right)}{\left(L_{s} I_{q S}+L_{m} I_{q r}^{\prime}\right)}+R_{s} L_{r}^{\prime}\right], a^{\prime}{ }_{56}=\frac{1}{k_{s}}\left[-\frac{\left(R_{s} I_{d s}-V_{c g d}\right)\left(L_{m}^{2}-L_{s} L_{r}^{\prime}\right) L_{m} I_{q s}}{\left(L_{s} I_{q s}+L_{m} I_{q r}^{\prime}\right)^{2}}-W_{r} L_{m} L_{r}^{\prime}\right], \\
& a_{59}^{\prime}=\frac{1}{k_{s}}\left[\frac{\left(L_{s} L_{r}^{\prime}-L_{m}^{2}\right) I_{q s}}{\left(L_{s} I_{q s}+L_{m} I_{q r}^{\prime}\right)}-L_{r}^{\prime}\right], a_{64}^{\prime}=\frac{1}{k_{s}}\left[-\frac{\left(R_{s} I_{d s}-V_{c g d}\right)\left(L_{s} L_{r}^{\prime}-L_{m}^{2}\right) L_{s} I_{d r}^{\prime}}{\left(L_{s} I_{q s}+L_{m} I_{q r}^{\prime}\right)^{2}}-R_{s} L_{m}\right], \\
& a_{65}^{\prime}=\frac{1}{k_{s}}\left[\frac{R_{s} I_{d r}^{\prime}\left(L_{s} L_{r}^{\prime}-L_{m}^{2}\right)}{\left(L_{s} I_{q s}+L_{m} I_{q r}^{\prime}\right)}-W_{r} L_{m} L_{s}\right], a_{66}^{\prime}=\frac{1}{k_{s}}\left[-\frac{\left(R_{s} I_{d s}-V_{c g d}\right)\left(L_{s} L_{r}^{\prime}-L_{m}^{2}\right) L_{m} I_{d r}^{\prime}}{\left(L_{s} I_{q s}+L_{m} I_{q r}^{\prime}\right)^{2}}+R_{r}^{\prime} L_{s}\right], \\
& a_{67}^{\prime}=\frac{1}{k_{s}}\left[\frac{\left(R_{s} I_{d s}-V_{c g d}\right)\left(L_{s} L_{r}^{\prime}-L_{m}^{2}\right)}{\left(L_{s} I_{q S}+L_{m} I_{q r}^{\prime}\right)}-W_{r} L_{s} L_{r}^{\prime}\right], a_{69}^{\prime}=\frac{1}{k_{s}}\left[\frac{\left(L_{m}^{2}-L_{s} L_{r}^{\prime}\right) I_{d r}^{\prime}}{\left(L_{s} I_{q S}+L_{m} I_{q r}^{\prime}\right)}\right], \\
& a_{74}^{\prime}=\frac{1}{k_{s}}\left[-\frac{\left(R_{s} I_{d s}-V_{c g d}\right)\left(L_{m}^{2}-L_{s} L_{r}^{\prime}\right) L_{s} I_{q r}^{\prime}}{\left(L_{s} I_{q s}+L_{m} I_{q r}^{\prime}\right)^{2}}+W_{r} L_{m} L_{s}\right], a_{75}^{\prime}=\frac{1}{k_{s}}\left[\frac{R_{s} I_{q r}^{\prime}\left(L_{m}^{2}-L_{s} L_{r}^{\prime}\right)}{\left(L_{s} I_{q s}+L_{m} I_{q r}^{\prime}\right)}-R_{s} L_{m}\right], \\
& a_{76}^{\prime}=\frac{1}{k_{s}}\left[-\frac{\left(R_{s} I_{d s}-V_{c g d}\right)\left(L_{m}^{2}-L_{s} L_{r}^{\prime}\right) L_{m} I_{q r}^{\prime}}{\left(L_{s} I_{q s}+L_{m} I_{q r}^{\prime}\right)^{2}}+\frac{\left(R_{s} I_{d s}-V_{c g d}\right)\left(L_{m}^{2}-L_{s} L_{r}^{\prime}\right)}{\left(L_{s} I_{q s}+L_{m} I_{q r}^{\prime}\right)}+W_{r} L_{s} L_{r}^{\prime}\right], \\
& a_{79}^{\prime}=\frac{1}{k_{s}}\left[\frac{\left(L_{s} L_{r}^{\prime}-L_{m}^{2}\right) I_{q r}^{\prime}}{\left(L_{s} I_{q s}+L_{m} I_{q r}^{\prime}\right)}+L_{m}\right], a_{84}^{\prime}=-\frac{1}{C_{g}}+\frac{\left(R_{s} I_{d s}-V_{c g d}\right) V_{c g d} L_{s}}{\left(L_{s} I_{q s}+L_{m} I_{q r}^{\prime}\right)^{2}}, a_{85}^{\prime}=-\frac{V_{c g d} R_{s}}{\left(L_{s} I_{q s}+L_{m} I_{q r}^{\prime}\right)}, \\
& a_{86}^{\prime}=\frac{\left(R_{s} I_{d s}-V_{c g d}\right) V_{c g d} L_{m}}{\left(L_{s} I_{q s}+L_{m} I_{q r}^{\prime}\right)^{2}}, a_{89}^{\prime}=\frac{2 V_{c g d}-R_{s} I_{d s}}{L_{s} I_{q s}+L_{m} I_{q r}^{\prime}}, a_{94}^{\prime}=-\frac{\left(R_{s} I_{d s}-V_{c g d}\right) V_{c g q} L_{s}}{\left(L_{s} I_{q s}+L_{m} I_{q r}^{\prime}\right)^{2}},
\end{aligned}
$$




$$
\begin{aligned}
& a_{95}^{\prime}=-\frac{1}{C_{g}}+\frac{R_{s} V_{c g q}}{L_{s} I_{q s}+L_{m} I_{q r}^{\prime}}, a_{96}^{\prime}=-\frac{\left(R_{s} I_{d s}-V_{c g d}\right) V_{c g q} L_{m}}{\left(L_{s} I_{q s}+L_{m} I_{q r}^{\prime}\right)^{2}}, a_{99}^{\prime}=-\frac{V_{c g q}}{L_{s} I_{q s}+L_{m} I_{q r}^{\prime}}, \\
& b_{11}^{\prime}=\frac{1}{J_{m}}\left[0.0068 \rho \pi r^{3} V_{\omega}+\rho \pi r^{2}\left(\frac{120 V_{\omega}^{3}}{W_{m}^{2} r}-\frac{7.05 V_{\omega}^{2}}{W_{m}}\right) e^{\left(-\frac{21 V_{w}}{W_{m} r}+0.74\right)}-\frac{1}{W_{m}}\left(2 1 \rho \pi r \left(\frac{30 V_{\omega}^{4}}{W_{m}^{2} r}-\right.\right.\right. \\
& \left.\left.\left.\frac{2.35 V_{\omega}^{3}}{W_{m}}\right) e^{\left(-\frac{21 V_{w}}{W_{m} r}+0.74\right)}\right)\right] .
\end{aligned}
$$

\section{E.2 ES Subsystem}

\section{1) State-Space Equations}

where

$$
\frac{d}{d t} X_{b}=A_{b} X_{b}+B_{b} U_{b}
$$

\section{where}

$$
\begin{gathered}
X_{b}=\left[\begin{array}{lll}
i_{b a t} & v_{c_{b}} & i_{d c}
\end{array}\right]^{T} \\
U_{b}=\left[\begin{array}{lll}
v_{d} & v_{i n v} & d_{A}
\end{array}\right]^{T} \\
A_{b}=\left[\begin{array}{ccc}
a_{10,10} & -\frac{1}{L_{b}} & 0 \\
\frac{1}{c_{b}} & 0 & -\frac{1}{c_{b}}\left(2 d_{A}-1\right) \\
0 & \frac{1}{L_{d c}}\left(2 d_{A}-1\right) & 0
\end{array}\right] \\
B_{b}=\left[\begin{array}{ccc}
0 & 0 & 0 \\
0 & 0 & 0 \\
\frac{1}{L_{d c}} & -\frac{1}{L_{d c}} & 0
\end{array}\right]
\end{gathered}
$$

In (E.14), $a_{10,10}=-\frac{R_{o}}{L_{b}}\left(\left(1+A_{0}(1-S o C)\right)\right.$.

\section{2) Small-Signal Equations}

where

$$
\frac{d}{d t} \Delta X_{b}=A_{b}^{\prime} \Delta X_{b}+B_{b}^{\prime} \Delta U_{b}
$$

$$
\begin{aligned}
\Delta X_{b} & =\left[\begin{array}{lll}
\Delta i_{b a t} & \Delta v_{c_{b}} & \Delta i_{d c}
\end{array}\right]^{T} \\
\Delta U_{b} & =\left[\begin{array}{lll}
\Delta v_{d} & \Delta v_{i n v} & \Delta d_{A}
\end{array}\right]^{T}
\end{aligned}
$$




$$
\begin{gathered}
A_{b}^{\prime}=\left[\begin{array}{ccc}
a_{10,10} & -\frac{1}{L_{b}} & \mathbf{0} \\
\frac{1}{c_{b}} & \mathbf{0} & -\frac{1}{c_{b}}\left(2 D_{A}-1\right) \\
\mathbf{0} & \frac{1}{L_{d c}}\left(2 D_{A}-1\right) & 0
\end{array}\right] \\
B_{b}^{\prime}=\left[\begin{array}{ccc}
0 & 0 & 0 \\
0 & 0 & -\frac{2}{c_{b}} I_{d c} \\
\frac{1}{L_{d c}} & -\frac{1}{L_{d c}} & \frac{2}{L_{d c}} V_{c_{b}}
\end{array}\right]
\end{gathered}
$$

\section{E.3 CSI-Load Subsystem}

\section{1) State-Space Equations}

$$
\frac{d}{d t} X_{c}=A_{c} X_{c}+B_{c} U_{c}
$$

where

$$
\begin{aligned}
& X_{C}=\left[\begin{array}{llllllll}
v_{c i d}^{+} & v_{c i q}^{+} & v_{c i d}^{-} & v_{c i q}^{-} & x_{1 m}^{+} & x_{2 m}^{+} & x_{1 m}^{-} & x_{2 m}^{-}
\end{array}\right]^{T} \\
& U_{C}=\left[\begin{array}{lllll}
i_{d c} & m_{i d}^{+} & m_{i q}^{+} & m_{i d}^{-} & m_{i q}^{-}
\end{array}\right]^{T} \\
& A_{C}=\left[\begin{array}{cccccccc}
0 & \omega_{L} & 0 & 0 & 0 & -Y_{P}^{+}\left(d^{2}+\omega_{o}^{2}\right) / C_{i} & 0 & 0 \\
-\omega_{L} & 0 & 0 & 0 & 0 & Y_{Q}^{+}\left(d^{2}+\omega_{o}^{2}\right) / C_{i} & 0 & 0 \\
0 & 0 & 0 & -\omega_{L} & 0 & 0 & 0 & -Y_{Q_{p d}}^{-}\left(d^{2}+\omega_{o}^{2}\right) / C_{i} \\
0 & 0 & \omega_{L} & 0 & 0 & 0 & 0 & Y_{Q_{p q}}^{-}\left(d^{2}+\omega_{o}^{2}\right) / C_{i} \\
1 & 0 & 0 & 0 & 0 & -\left(d^{2}+\omega_{o}^{2}\right) & 0 & 0 \\
0 & 0 & 0 & 0 & 1 & 2 d & 0 & 0 \\
1 & 0 & 0 & 0 & 0 & 0 & 0 & -\left(d^{2}+\omega_{o}^{2}\right) \\
0 & 0 & 0 & 0 & 0 & 0 & 1 & 2 d
\end{array}\right]
\end{aligned}
$$




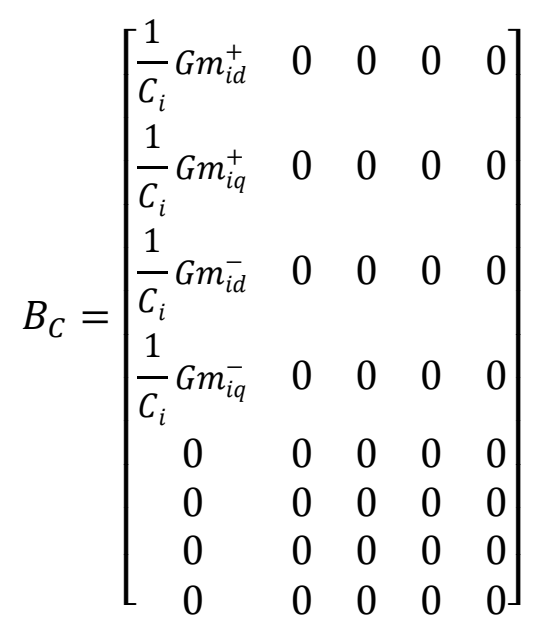

\section{2) Small-Signal Equations}

$$
\frac{d}{d t} \Delta X_{c}=A_{c}^{\prime} \Delta X_{c}+B_{c}^{\prime} \Delta U_{c}
$$

where

$$
\begin{aligned}
& \Delta X_{C}=\left[\begin{array}{llllllll}
\Delta v_{\text {cid }}^{+} & \Delta v_{\text {ciq }}^{+} & \Delta v_{\text {cid }}^{-} & \Delta v_{\text {ciq }}^{-} & \Delta x_{1 m}^{+} & \Delta x_{2 m}^{+} & \Delta x_{1 m}^{-} & \Delta x_{2 m}^{-}
\end{array}\right]^{T} \\
& \Delta U_{c}=\left[\begin{array}{lllll}
\Delta i_{d c} & \Delta m_{i d}^{+} & \Delta m_{i q}^{+} & \Delta m_{i d}^{-} & \Delta m_{i q}^{-}
\end{array}\right]^{T} \\
& A_{c}^{\prime}=\left[\begin{array}{cccccccc}
0 & \omega_{L} & 0 & 0 & 0 & -Y_{P}^{+}\left(d^{2}+\omega_{o}^{2}\right) / C_{i} & 0 & 0 \\
-\omega_{L} & 0 & 0 & 0 & 0 & Y_{Q}^{+}\left(d^{2}+\omega_{o}^{2}\right) / C_{i} & 0 & 0 \\
0 & 0 & 0 & -\omega_{L} & 0 & 0 & 0 & -Y_{Q_{p d}}^{-}\left(d^{2}+\omega_{o}^{2}\right) / C_{i} \\
0 & 0 & \omega_{L} & 0 & 0 & 0 & 0 & Y_{Q_{p q}}^{-}\left(d^{2}+\omega_{o}^{2}\right) / C_{i} \\
1 & 0 & 0 & 0 & 0 & -\left(d^{2}+\omega_{o}^{2}\right) & 0 & 0 \\
0 & 0 & 0 & 0 & 1 & 2 d & 0 & 0 \\
1 & 0 & 0 & 0 & 0 & 0 & 0 & -\left(d^{2}+\omega_{o}^{2}\right) \\
0 & 0 & 0 & 0 & 0 & 0 & 1 & 2 d
\end{array}\right]
\end{aligned}
$$




$$
B_{c}^{\prime}=\left[\begin{array}{ccccc}
\frac{1}{C_{i}} G M_{i d}^{+} & \frac{1}{C_{i}} G I_{d c} & 0 & 0 & 0 \\
\frac{1}{C_{i}} G M_{i q}^{+} & 0 & \frac{1}{C_{i}} G I_{d c} & 0 & 0 \\
\frac{1}{C_{i}} G M_{i d}^{-} & 0 & 0 & \frac{1}{C_{i}} G I_{d c} & 0 \\
\frac{1}{C_{i}} G M_{i q}^{-} & 0 & 0 & 0 & \frac{1}{C_{i}} G I_{d c} \\
0 & 0 & 0 & 0 & 0 \\
0 & 0 & 0 & 0 & 0 \\
0 & 0 & 0 & 0 & 0 \\
0 & 0 & 0 & 0 & 0
\end{array}\right]
$$

\section{E.4 The Entire System}

\section{1) State-Space Equations}

where

$$
\frac{d}{d t} X=A X+B U
$$

$$
\begin{aligned}
& X=\left[\begin{array}{lllllllllll}
\omega_{m} & \omega_{r} & \delta \theta & i_{q s} & i_{d s} & i_{q r}^{\prime} & i_{d r}^{\prime} & v_{c g q} & v_{c g d} & i_{b a t} & v_{c_{b}}
\end{array}\right. \\
& \left.\begin{array}{lllllllll}
v_{c i d}^{+} & v_{c i q}^{+} & v_{c i d}^{-} & v_{c i q}^{-} & x_{1 m}^{+} & x_{2 m}^{+} & x_{1 m}^{-} & x_{2 m}^{-} & i_{d c}
\end{array}\right]^{T} \\
& U=\left[\begin{array}{lllllll}
v_{w} & d_{b} & d_{A} & m_{i d}^{+} & m_{i q}^{+} & m_{i d}^{-} & m_{i q}^{-}
\end{array}\right]^{T} \\
& B=\left[\begin{array}{ccccccc}
b_{11} & 0 & 0 & 0 & 0 & 0 & 0 \\
0 & 0 & 0 & 0 & 0 & 0 & 0 \\
0 & 0 & 0 & 0 & 0 & 0 & 0 \\
0 & 0 & 0 & 0 & 0 & 0 & 0 \\
0 & 0 & 0 & 0 & 0 & 0 & 0 \\
0 & 0 & 0 & 0 & 0 & 0 & 0 \\
0 & 0 & 0 & 0 & 0 & 0 & 0 \\
0 & 0 & 0 & 0 & 0 & 0 & 0 \\
0 & 0 & 0 & 0 & 0 & 0 & 0 \\
0 & 0 & 0 & 0 & 0 & 0 & 0 \\
0 & 0 & 0 & 0 & 0 & 0 & 0 \\
0 & 0 & 0 & 0 & 0 & 0 & 0 \\
0 & 0 & 0 & 0 & 0 & 0 & 0 \\
0 & 0 & 0 & 0 & 0 & 0 & 0 \\
0 & 0 & 0 & 0 & 0 & 0 & 0 \\
0 & 0 & 0 & 0 & 0 & 0 & 0 \\
0 & 0 & 0 & 0 & 0 & 0 & 0 \\
0 & 0 & 0 & 0 & 0 & 0 & 0 \\
0 & 0 & 0 & 0 & 0 & 0 & 0 \\
0 & 0 & 0 & 0 & 0 & 0 & 0
\end{array}\right]
\end{aligned}
$$




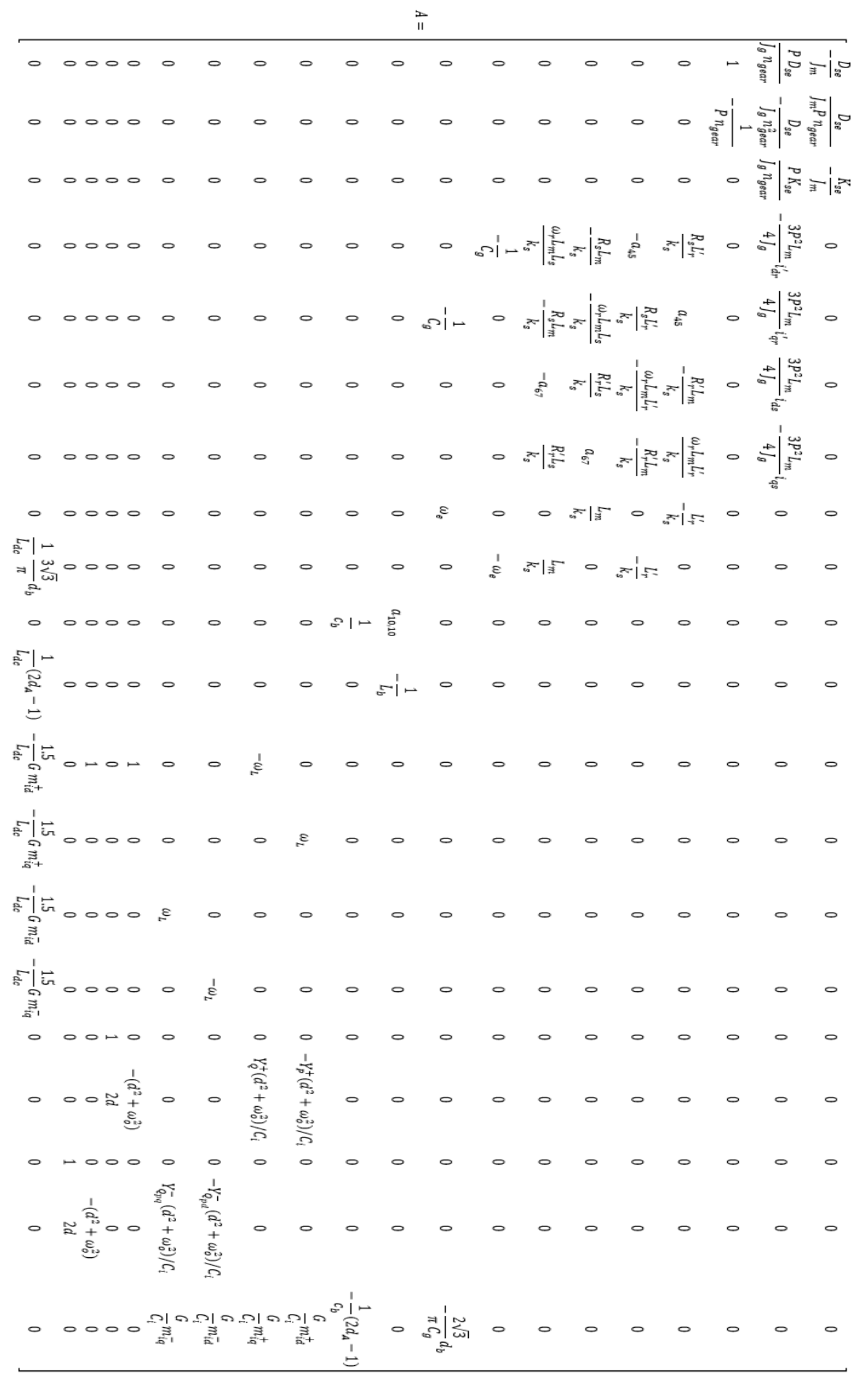




\section{2) Small-Signal Equations}

$$
\frac{d}{d t} \Delta X=A^{\prime} \Delta X+B^{\prime} \Delta U
$$

where

$$
\begin{gathered}
\Delta X=\left[\begin{array}{llllll}
\Delta \omega_{m} & \Delta \omega_{r} \Delta \delta \theta & \Delta i_{q s} & \Delta i_{d s} \Delta i_{q r}^{\prime} \Delta i_{d r}^{\prime} \Delta v_{c g q} \Delta v_{c g d} \\
\Delta i_{b a t} \Delta v_{c_{b}} \Delta v_{c i d}^{+} \Delta v_{c i q}^{+} \Delta v_{c i d}^{-} \Delta v_{c i q}^{-} \Delta x_{1 m}^{+} \Delta x_{2 m}^{+} \Delta x_{1 m}^{-} \Delta x_{2 m}^{-} \Delta i_{d c}
\end{array}\right]^{T}
\end{gathered}
$$

$$
\Delta U=\left[\begin{array}{llllll}
\Delta v_{\omega} & \Delta d_{b} & \Delta d_{A} & \Delta m_{i d}^{+} & \Delta m_{i q}^{+} \Delta m_{i d}^{-} & \Delta m_{i q}^{-}
\end{array}\right]^{T}
$$

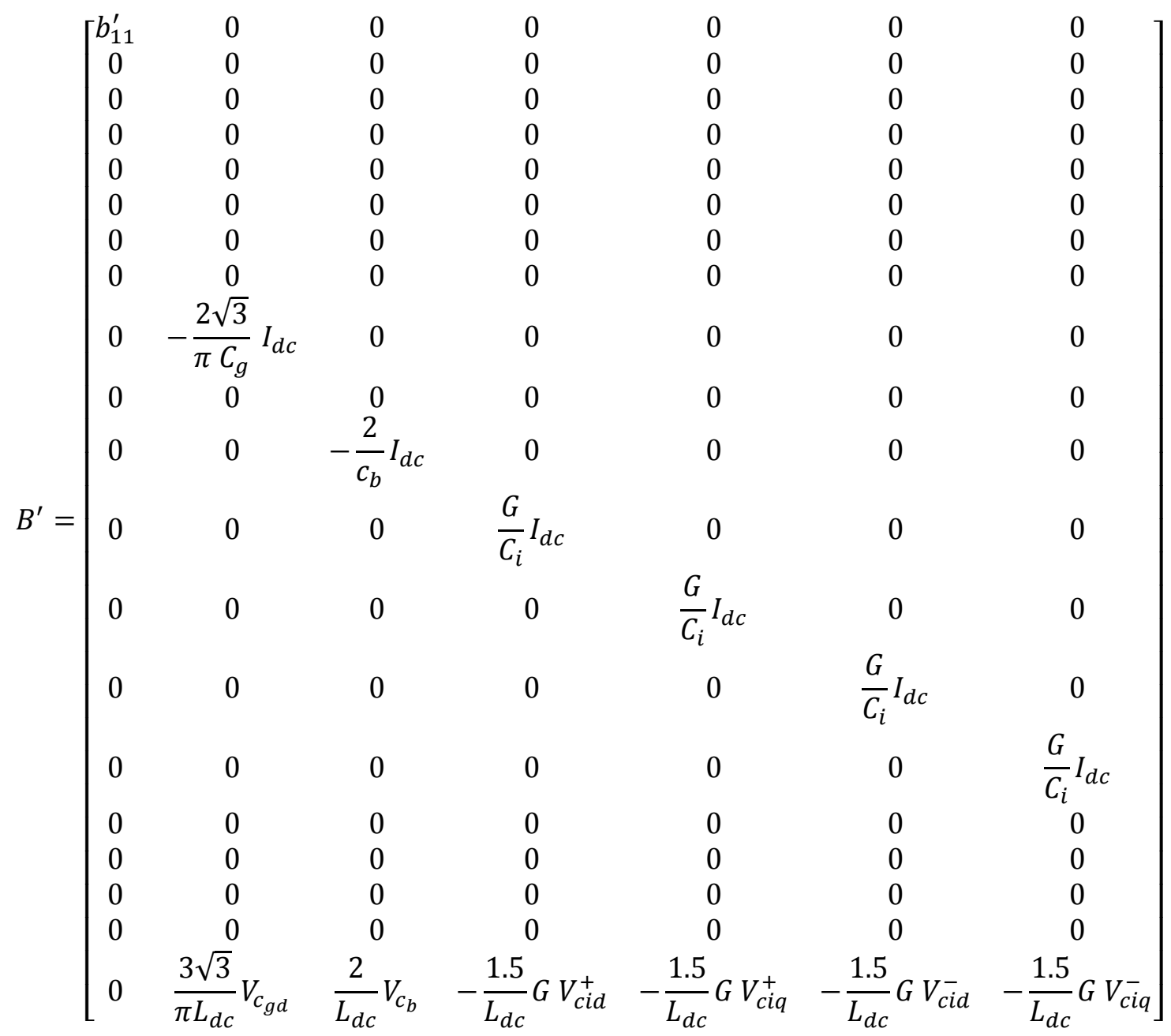




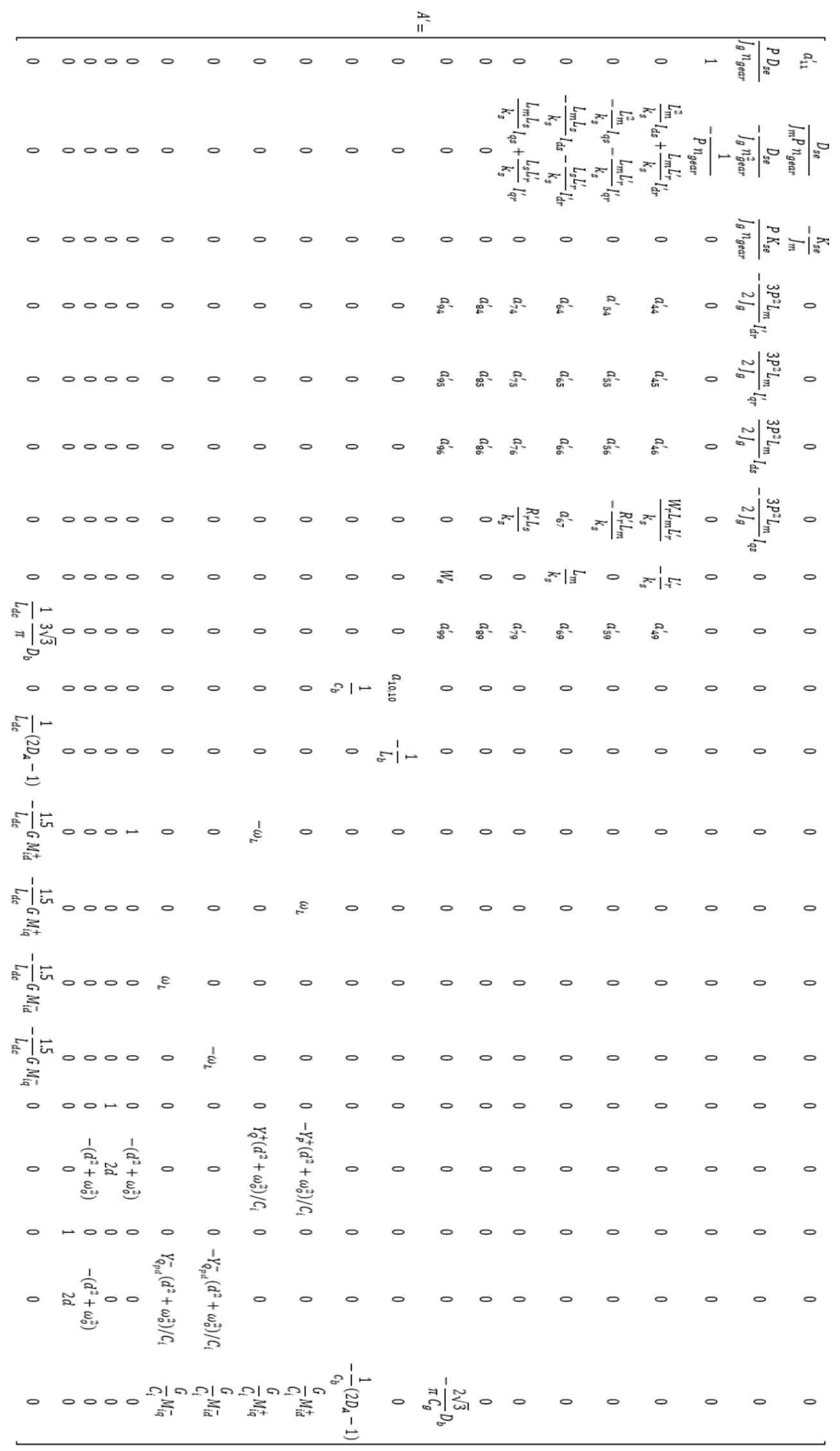




\section{References}

[1] Global Wind Energy Council GWEC Annual Report 2015. Available at: http://www.gwec.net/wp-content/uploads/vip/GWEC-Global-Wind-2015-Report_April2016_19_04.pdf. Last accessed on June $21^{\text {st }} 2016$.

[2] World Wind Energy Association. 2015 Small Wind World Report. Available at: http://small-wind.org/wp-content/uploads/2014/12/Summary_SWWR2015_online.pdf. Last accessed on June $21^{\text {st }} 2016$.

[3] U.S. Department of Energy. 2014 Distributed Wind Market Report. Available at: http://energy.gov/sites/prod/files/2015/08/f25/2014-Distributed-Wind-Market-Report8.7_0.pdf. Last accessed on June $21^{\text {st }} 2016$.

[4] The Canadian Wind Energy Association. Canadian Market. Available at: http://canwea.ca/wind-energy/installed-capacity/. Last accessed on June $21^{\text {st }} 2016$.

[5] The International Energy Agency 2011. IEA Report. China Wind Energy Development Roadmap 2050. Available at:

https://www.iea.org/publications/freepublications/publication/China_Wind_Roadmap_S

ummary_Web.pdf. Last accessed on June $21^{\text {st }} 2016$.

[6] Global Off-Grid Lighting Association (GOGLA). Available at: https://www.gogla.org/. Last accessed on June $21^{\text {st }} 2016$.

[7] Aboriginal Affairs and Northern Development Canada. Available at: http://www.aadncaandc.gc.ca/eng/1314295992771/1314296121126. Last accessed on June $21^{\text {st }} 2016$.

[8] S. Misak, and L. Prokop, "Off-grid power systems," $9^{\text {th }}$ International Conference on Environment and Electrical Engineering, 2010, pp.14-17.

[9] J. Brunarie, G. Myerscough, A. Nystrom, and J. Ronsen, "Delivering cost savings and environmental benefits with hybrid power," $31^{\text {st }}$ International Telecommunications Energy Conference, 2009, pp.1-9.

[10] J.K. Kaldellis. Stand-Alone and Hybrid Wind Energy Systems - Technology, Energy Storage and Applications. 1st ed. UK. Woodhead Publishing limited, 2010.

[11]P. Sharma, T.S. Bhatti, and K.S.S Ramakrishna, "Control of reactive power of autonomous wind-diesel hybrid power systems," Joint International Conference on Power Electronics, Drives and Energy Systems \& Power, India 2010, pp. 1-6. 
[12] F. Blaabjerg, Z. Chen, R. Teodorescu, and F. Iov, "Power electronics in wind turbine systems," IEEE $5^{\text {th }}$ International Power Electronics and Motion Control Conference, 2006, vol. 1, pp. 1-11.

[13]Z. Chen, J.M. Guerrero, and F. Blaabjerg, "A review of the state of the art of power electronics for wind turbines," IEEE Transactions on Power Electronics, vol. 24,no. 8, pp. 1859 - 1875, Aug. 2009.

[14] K.W.E.Cheng, J.K. Lin, Y.J. Bao, and XD Xue, "Review of the wind energy generating system," $8^{\text {th }}$ International Conference on Advances in Power System Control, Operation and Management, 2009, pp. 1-7.

[15] B. Wu, Y. Lang, N. Zargari, and S. Kouro. Power conversion and control of wind energy systems. 1st ed. New Jersey, USA, Wiley, 2011.

[16] Y. Kawabata, T. Oka, E. Ejiogu , and T. Kawabata, "Variable speed constant frequency stand-alone power generator using wound-rotor induction machine," $4^{\text {th }}$ international conference on power Electronics and Motion Control, 2004, vol. 3, pp.1778 - 1784.

[17] B.A. Zahir, J.G. Kettleborough, and I.R. Smith, "A standalone induction generator model producing a constant voltage constant frequency output, " $4^{\text {th }}$ International Conference on Emerging Technologies, 2008, pp. 83 - 86.

[18] Y. Amirat, M.E.H. Benbouzid, B. Bensaker, R. Wamkeue, and H. Mangel, "The state of the art of generators for wind energy conversion systems," Proceedings of the International Conference on Electrical Machines, China 2006, pp. 1-6.

[19]H. Li, and Z. Chen, "Overview of different wind generator systems and their comparisons," IET Renewable Power Generation, vol. 2,no. 2, pp.123-138, June 2008.

[20] R. Cardenas , R. Pena, J. Proboste , G. Asher, and J. Clare, "MRAS observer for sensorless control of standalone doubly fed induction generators," IEEE Transactions on Energy Conversion, vol. 20,no. 4, pp.710-718, Dec. 2005.

[21] M. Aktarujjaman, M.A. Kashem, M. Negnevitsky, and G. Ledwich, "Control stabilisation of an islanded system with DFIG wind turbine," $1^{\text {st }}$ International Power and Energy Conference, Malaysia 2006, pp. 312 - 317.

[22] N. Mendis, K.M. Muttaqi, S. Sayeef, and S. Perera, "Standalone operation of wind turbinebased variable speed generators with maximum power extraction capability," IEEE Transactions on Energy Conversion, vol. 27, no. 4, pp. 822-834, Dec. 2012. 
[23] S.Z. Farooqui, "Autonomous wind turbines with doubly-Fed induction generators," $3^{\text {rd }}$ International Conference on Energy and Environment, 2009, pp. 62 - 70.

[24] R.A. McMahon, P.C. Robets , X. Wang, and P.J. Tavner, "Performance of BDFM as generator and motor," IEE Proceedings Electric Power Applications, 2006, vol. 153, no.2, pp. 289-299.

[25] K. Protsenko, and X. Dewei. "Modeling and control of brushless doubly-fed induction generators in wind energy applications," IEEE Transactions on Power Electronics, vol.23,no. 3, pp.1191 - 1197, May.2008.

[26] I. Azmy, A. Abdel-Khalik, A.M. Massoud, and S. Ahmed, “Assessment of fault-ride through capability of grid-connected brushless DFIG wind turbines," IET Conference on Renewable Power Generation, 2011, pp. 1-7.

[27]H.S. Kim, and D.D.C Lu, "Review on wind turbine generators and power electronic converters with the grid-connection issues," $20^{\text {th }}$ Australasian Universities Power Engineering Conference, 2010, pp. 1-6.

[28] S. Ademi, and M. Jovanovic, "Vector control strategies for brushless doubly-fed reluctance wind generators," 2nd International Symposium on Environment Friendly Energies and Applications, 25-27 June 2012, pp. $44-49$.

[29] C. Grantham, and D. Seyoum, "The dynamic characteristics of an isolated self-excited induction generator driven by a wind turbine," International Conference on Electrical Machines and Systems, 2008, pp. 2351 - 2356.

[30] S. Hazra, and P.S. Sensarma, "Self-excitation and control of an induction generator in a stand-alone wind energy conversion system," IET Renewable Power Generation, vol. 4,no. 4, pp. $383-393,2010$.

[31] G.V. Jayaramaiah, and B.G. Fernandes, "Novel voltage controller for standalone induction generator using PWM-VSI," IEEE International Conference on Industry Applications, 2006, vol.1, pp.204-208.

[32] J.A. Barrado, R. Girno, and H. Valderrama, "Standalone self-excited induction generator with a three-phase four-wire active filter and energy storage system," IEEE International Symposium on Industrial Electronics, 2007, pp. 600-605. 
[33] A.M. Sharaf, A.S. Aljankawey, and I.H. Altas, "Dynamic voltage stabilization of standalone wind energy schemes," IEEE Canada Electrical Power Conference, 2007, pp. 1419.

[34]B. Singh, and G.K. Kasal, "Solid state voltage and frequency controller for a standalone wind power generating system," IEEE Transactions on Power Electronics, vol. 23,no. 3, pp. 1170 - 1177, May 2008.

[35]B.V. Perumal, and J.K. Chatterjee, "Voltage and frequency control of a standalone brushless wind electric generation using generalized impedance controller," IEEE Transactions on Energy Conversion, vol. 23,no. 2, pp. 632-641, June 2008.

[36] V. Vongmanee, "Emulator of wind turbine generator using dual inverter controlled squirrel cage induction motor," International Conference on Power Electronics and Drive Systems, 2009, pp. 1313 - 1316.

[37] G.K. Kasal, and B. Singh, "Voltage and frequency controllers for an asynchronous generator-based isolated wind energy conversion system, " IEEE Transactions on Energy Conversion, vol. 26, no. 2, pp.402 - 416. June 2011.

[38] S. Sharma, and B. Singh. "Variable speed stand-alone wind energy conversion system using synchronous generator," International Conference on Power and Energy Systems, 2011, pp.1-6.

[39] A. Doria-Cerezo ,V.I. Utkin, R.S. Munoz-Aguilar, and E. Fossas, "Control of a standalone wound rotor synchronous generator: two sliding mode approaches via regulation of the d-voltage component," IEEE Transactions on Control Systems Technology, vol. 20, no.3, pp.779 - 786, May 2012.

[40] H. Polinder, "Overview of and trends in wind turbine generator systems," IEEE Power and Energy Society General Meeting, 2011, pp.1-8.

[41] H. Haraguchi, S. Morimoto, and M. Sanada, "Suitable design of a PMSG for a small-scale wind power generator," International Conference on Electrical Machines and Systems, 2009, pp. 1-6.

[42] M.E. Haque, K.M. Muttaqi, and M. Negnevitsky, "Control of a standalone variable speed wind turbine with a permanent magnet synchronous generator," IEEE Power and Energy Society General Meeting, 2008, pp.1-9. 
[43] M. Fatu, L. Tutelea, I. Boldea, and R. Teodorescu, "Novel motion sensorless control of standalone permanent magnet synchronous generator (PMSG): harmonics and negative sequence voltage compensation under nonlinear load," European Conference on Power Electronics and Applications, Aalborg, Denmark 2007, pp.1-10.

[44] C.N. Bhende, "Stand-alone wind energy supply system," International Conference on Power Systems, 2009, pp. 1-6.

[45] M.E. Haque, M. Negnevitsky, and K.M. Muttaqi, “A novel control strategy for a variablespeed wind turbine with a permanent-magnet synchronous generator," IEEE Transactions on Industry Applications, vol. 46, no.1, pp. 331-339, Jan-Feb. 2010.

[46]F. Kendouli, K. Abed, K. Nabti, H. Benalla, and B. Azoui, "High performance PWM converter control based PMSG for variable speed wind turbine," $1^{\text {st }}$ International Conference on Renewable Energies and Vehicular Technology, 2012, pp. 502-507.

[47] R. Mittal, K.S. Sandhu, and D.K. Jain, "Battery energy storage system for variable speed driven PMSG for wind energy conversion system," Joint International Conference on Power Electronics, Drives and Energy Systems \& Power, India 2010, pp.1-5.

[48] M. Hilmy, M. Orabi, M.E. Ahmed, M. El-Nemr, and M. Youssef, "A less sensor control method for standalone small wind energy using permanent magnet synchronous generator," 26 $6^{\text {th }}$ Annual IEEE Applied Power Electronics Conference and Exposition, 2011, pp.1968-1974.

[49] C.N. Bhende, S. Mishra, and S.G. Malla, "Permanent magnet synchronous generatorbased standalone wind energy supply system," IEEE Transactions on Sustainable Energy, vol. 2, no.4, pp.361-373, Oct. 2011.

[50] T. Fukami, K. Nakagawa, Y. Kanamaru, and T. Miyamoto, "A technique for the steadystate analysis of a grid-connected permanent magnet induction generator," IEEE Transactions on Energy Conversion, vol. 19, no. 2, pp. 318-324, June 2004.

[51]T. Tsuda, T. Fukami, Y. Kanamaru, and T. Miyamoto, "Performance analysis of the permanent-magnet induction generator under unbalanced grid voltages," Electrical Engineering in Japan, vol. 161, no.4, pp. 60-69, 2007.

[52]P. Sharma, and T.S. Bhatti, "Performance investigation of isolated wind-diesel hybrid power system with WECS having PMIG," IEEE Transactions on Industrial Electronics, vol. 60, no. 4, pp. 1630-1637, Apr. 2013. 
[53] T. Epskamp, B. Hagenkort, T. Hartkopf , and S. Jöckel, "No gearing no converter assessing the idea of highly reliable permanent-magnet induction generators," Proceedings of European Wind Energy Conference, Nice, France 1999, pp. 813-816.

[54] Qingdao Hengfeng Wind Power Generator Co., Ltd. NO. No.226 Taishan Road,Jiaonan City,Shandong Province,China.

[55] AllEarth Renewables. 94 Harvest Lane, Williston, Vermont 05495, USA.

[56] R. Karthikeyan, K. Vijayakumar, R. Arumugam, and V. Kamaraj, "Design and analysis of a switched reluctance generator for rural electrification in stand alone wind energy conversion system," International Conference on Power Systems, 2009, pp. 1-6.

[57]H.K. Karegar, M. Yazdi, and A. Siadatan, "New structure for high speed and variable speed wind turbine based switched reluctance generator," IEEE International Conference on Power and Energy, 2010, pp. 200-205.

[58] D. McSwiggan, L. Xu, and T. Littler, "Modeling and control of a variable-speed switched reluctance generator based wind turbine," $42^{\text {nd }}$ International Universities Power Engineering Conference, 2007, pp. 459-463.

[59] X. Zhang, G. Tan, S. Kuai, and Q. Wang, "Position sensorless control of switched reluctance generator for wind energy conversion," Power and Energy Engineering Conference, Asia-Pacific 2010, pp. 1-5.

[60] T. Yamaguchi, N. Yamamura, and M. Ishida, "Study for small size wind power generating system using switched reluctance generator," $37^{\text {th }}$ Annual Conference on IEEE Industrial Electronics Society, 2011, pp. 967-972.

[61] A. Stabile, A.J.M. Cardoso, and C. Boccaletti, "Efficiency analysis of power converters for urban wind turbine applications," IEEE International Conference on Sustainable Energy Technologies, Dec 6-9, 2010. pp.1-6

[62] W. Liang, and W. Liu, "Key technologies analysis of small scale non-grid-connected wind turbines: A review," World Non-Grid-Connected Wind Power and Energy Conference, 2010, pp. 1-6.

[63]F. Blaabjerg, M. Liserre, and K. Ma "Power electronics converters for wind turbine systems," IEEE Transactions on Industry Applications, vol. 48, no.2,pp.708-719. MarApr. 2012. 
[64]D.S. Oliveira, M.M. Reis, C. Silva, L. Colado Barreto, F. Antunes, and B.L. Soares, "A three-phase high frequency semicontrolled rectifier for PM WECS," IEEE Transactions on Power Electronics, vol. 25, no. 3, pp. 677-685, Mar. 2010.

[65] A.M.O. Haruni, M. Negnevitsky, M.E. Haque, and A. Gargoom, "Control strategy of a stand-alone variable speed wind turbine with integrated energy storage system using NPC converter," IEEE Power and Energy Society General Meeting, 2011, pp.1-8.

[66]F. Blaabjerg, K. Ma, and D. Zhou, "Power electronics and reliability renewable energy systems," IEEE International Symposium on Industrial Electronics, 2012, pp. 19-30.

[67] M. Malinowski, K. Gopakumar , J. Rodriguez, and M.A. Pérez, “A Survey on cascaded multilevel inverters," IEEE Transactions on Industrial Electronics, vol. 57, no.7, pp. 2197-2206, July 2010.

[68] S. Kouro, et al., "Recent advances and industrial applications of multilevel converters," IEEE Transactions on Industrial Electronics, vol. 57, no. 8, pp. 2553-2580, Aug.2010.

[69] J. Rodriguez, S. Bernet, P.K. Steimer, and I.E. Lizama, "A Survey on neutral-pointclamped inverters," IEEE Transactions on Industrial Electronics, vol. 57, no. 7, pp. 22192230, July 2010.

[70] N. Stretch, and M. Kazerani, "A Stand-alone, split-phase current-sourced inverter with novel energy storage," IEEE Transactions on Power Electronics, vol.23, no.6, pp. 2766 2774, Nov. 2008.

[71]M. Kazerani, and Y. Ye, "Comparative evaluation of three-phase PWM voltage- and current-source converter topologies in FACTS applications," Proceedings of 2002 IEEE Power Engineering Society Summer Meeting, July 2002, Chicago, USA, vol. 1, pp. 473479.

[72] Y. Ye, M. Kazerani, and V. H. Quintana, "Current-source converter-based STATCOM: modeling and control," IEEE Transaction of Power Delivery, vol. 20, no.2, pp. 795-800. Apr. 2005.

[73] X. Tan, J. Dai and W. Bin, "A novel converter configuration for wind applications using PWM CSI with diode rectifier and buck converter," in Proc. IEEE Int. Electr. Machines \& Drives Conf., 2011, pp. 359-364. 
[74]Dai, Jingya, "Current source converters for megawatt wind energy conversion systems" $\mathrm{PhD}$ thesis at University of Ryerson, Toronto, CANADA (2010). Available at: http://digitalcommons.ryerson.ca/dissertations/841. Last accessed on June $21^{\text {st }} 2016$.

[75] F.Z. Peng, “Z-source inverter," IEEE Transaction on Industrial Applications, vol.39, no.2, pp. 504-510, Mar-Apr. 2003.

[76] S. Sonar, and T. Maity," Z-source inverter based control of wind power," International Conference on Energy, Automation, and Signal, 28-30 Dec. 2011, pp. 1-6.

[77] B.K. Ramasamy, A. Palaniappan, and ,S.M Yakoh, "Direct-drive low-speed wind energy conversion system incorporating axial-type permanent magnet generator and Z-source inverter with sensorless maximum power point tracking controller," IET Renewable Power Generation, vol.7 no.3, pp.284-295. May 2013.

[78]Z. Alnasir, and M. Kazerani, "Standalone SCIG-based wind energy conversion system using Z-source inverter with energy storage integration," IEEE $27^{\text {th }}$ Canadian Conference on Electrical and Computer Engineering, May 2014, pp. 1-6.

[79] P. Wheeler, J. Clare, L. Empringham, M. Apap, and M. Bland, "Matrix converters," Power Engineering Journal, vol. 16, no. 6, pp. 273-282. 2002.

[80] J. Mahlein, J. Igney, J. Weigold, M. Braun, and O. Simon, "Matrix converter commutation strategies with and without explicit input voltage sign measurement," IEEE Transactions on Industrial Electronics, vol. 49, no. 2, pp. 407-414, Apr. 2002.

[81]G. Yang, and Y. Zhu, "Application of a matrix converter for PMSG wind turbine generation system," $2^{\text {nd }}$ IEEE International Symposium on Power Electronics for Distributed Generation Systems, 2010, pp.185-189

[82]L. Wei, and T.A. Lipo, "A novel matrix converter topology with simple commutation," $36^{\text {th }}$ IEEE Industry Application Society Conference, 2001, vol. 3, pp. 1749 - 1754.

[83] S.M. Barakati, M. Kazerani, and X. Chen, "A new wind turbine generation system based on matrix converter," IEEE Power Engineering Society General Meeting, 12-16 June 2005, vol.3 , pp. 2083 - 2089.

[84] A. Yarahmadi , D.A. Khaburi, and H. Behnia, "Direct Virtual Torque Control of DFIG grid connection using Indirect Matrix Converter," $3^{\text {rd }}$ conference of Power Electronics and Drive Systems Technology, 15-16 Feb. 2012, pp. 115 - 120. 
[85]H. Wang, M. Liserre and F. Blaabjerg, "Toward reliable power electronics - challenges, design tools and opportunities," IEEE Industrial Electronics Magazine, vol. 7, no. 2, pp. 17-26, Jun. 2013.

[86]E. Wolfgang, "Examples for failures in power electronics systems," presented at ECPE Tutorial on Reliability Power Electronic System, Nuremberg, Germany, Apr. 2007.

[87] S. Yang, A. Bryant, P. Mawby, D. Xiang, L. Ran and P. Tavner, "An industry-based survey of reliability in power electronic converters," IEEE Trans. Ind. Appl., vol. 47, no. 3, pp. 1441-1451, May/Jun. 2011.

[88] C. Busca, R. Teodorescu, F. Blaabjerg, S. M. Nielsen, L. Helle, T. Abeyasekera, and P. Rodriguez, "An overview of the reliability prediction related aspects of high power IGBTs in wind power applications," Microelectronics Reliability, vol. 51, no. 9-11, pp. 19031907, Sep./Nov. 2011.

[89] L. Chen, F. Z. Peng and D. Cao, "A smart gate drive with self-diagnosis for power MOSFETs and IGBTs," Proceedings of 23rd Annual IEEE Applied Power Electronics Conference and Exposition (APEC2008), pp.1602- 1607, 2008.

[90] M. A. Rodriguez, A. Claudio, D. Theilliol and L. G. Vela, "A new fault detection technique for IGBT based on gate voltage monitoring," in Proc. IEEE Power Electron. Spec. Conf., pp. 1001-1005, 2007.

[91] M.T. Rahimo and N.Y.A. Shammas, "Freewheeling diode reverse-recovery failure modes in IGBT applications", IEEE Trans. on Industry Applications, vol. 37, no. 2, pp. 661-670, Mar./Apr. 2001.

[92]R.Wu., F. Blaabjerg, H.Wang and M.Liserre, "Overview of catastrophic failures of freewheeling diodes in power electronic circuits," Journal of Microelectronic Reliability ELSEVIER, Sep.-Nov. 2013, pp. 1788-1792.

[93] Qingdao Zeyu Wind Power Generator Co., Ltd., Tieshan Industry Park, Jiaonan District, Qingdao, Shandong, China. Postal Code 266400. Available at: http://www.globalsources.com/si/AS/Qingdao-Zeyu/6008833217246/Homepage.htm. Last accessed on June $21^{\text {st }} 2016$.

[94]B. Sahan, S. Ara'ujo, C. N"oding and P. Zacharias, "Comparative Evaluation of ThreePhase Current Source Inverters for Grid Interfacing of Distributed and Renewable Energy Systems," IEEE Trans. Power Electronics, vol. 26, no. 8, pp. 2304-2318, Aug. 2011. 
[95]L. Bin and S.K. Sharma, "A Literature Review of IGBT Fault Diagnostic and Protection Methods for Power Inverters," IEEE Trans. on Industry Applications, vol. 45, no. 5, pp. 1770-1777, Sept.-Oct. 2009.

[96] M.S. Hamad and A.K. Abdelsalam, "Over-voltage protection of single phase grid connected current source inverters using a simplified passive network," IET conf. on Renewable Power Generation, Sept. 6-8, 2011, pp. 1-5.

[97] M. Swierczynski, R. Teodorescu, C.N. Rasmussen, P. Rodriguez, and H. Vikelgaard, "Overview of the energy storage systems for wind power integration enhancement," IEEE International Symposium on Industrial Electronics, 2010, pp. 3749-3756.

[98] H. Chen et. al., "Progress in electrical energy storage system: a critical review," Progress in natural science ELSEVIER, pp. 291-312, July 2008.

[99] L. Barote, and C. Marinescu, "Storage analysis for stand-alone wind energy applications," $12^{\text {th }}$ International Conference on Optimization of Electrical and Electronic Equipment, 2010, pp. 1180-1185.

[100] N. Mendis, K.M. Muttaqi, and S. Perera, "Active power management of a super capacitorbattery hybrid energy storage system for standalone operation of DFIG based wind turbines," IEEE Industry Applications Society Annual Meeting, 7-11 Oct. 2012, pp. 1 - 8.

[101] P. Sivachandran, P. Venkatesh, and N. Kamaraj, "A review of wind energy based decentralized power generation systems with new developments in India," Journal of Energy \& Environment,vol. 6,pp.102-107, May.2007.

[102] Z. Alnasir, and M. Kazerani, "An analytical literature review of stand-alone wind energy conversion system from generator viewpoint," Renewable and Sustainable Energy Reviews, vol. 28, issue. C, pp. 597-615, 2013.

[103] P.K. Sen, and J.P. Nelson, "Application guidelines for induction generators," International Conference on Electrical Machines and Drives, 1997,pp. WC1/5.1WC1/5.3.

[104] F. Spinato, P.J. Tavner, G. Bussel, and E. Koutoulakos, "Reliability of wind turbine subassemblies," IET Renewable Power Generation, vol. 3, no. 4, pp. 387- 401, 2009.

[105] J. Ribrant, and L. Bertling, "Survey of failures in wind power systems with focus on Swedish wind power plants during 1997-2005," IEEE Power Engineering Society General Meeting, 2007, pp. 1-8. 
[106] P.J. Tavnet, G. Bussel, and F. Spinato, "Machine and converter reliabilities in wind turbines," $3^{\text {rd }}$ IET International Conference on Power Electronics, Machines and Drives 2006, pp. 127-130.

[107] E. Echavarria, B. Hahn, G.J.W. Brussel, and T. Tomiyama, "Reliability of wind turbine technology through time," Journal of Solar Energy Engineering, vol. 30, no. 3, pp. 0310051-0310058, 2008.

[108] G. Zhenhong, and C. Liuchen, "Calculation and study on cogging torque of small wind turbine PMSG," Canadian Conference on Electrical and Computer Engineering, 2008, pp. $000589-000594$.

[109] J. Puranen, "Induction motor versus permanent magnet synchronous motor in motion control applications: a comparative study," $\mathrm{PhD}$ thesis at University of Technology, Lappeenranta, Finland 2006.

[110] G. Cheng, and Y. Xu, "A statistical model for gears noise prediction in gearbox," International Conference on Electrical and Control Engineering, 2010, pp. 270 - 272.

[111] Nanjing Supermann Industrial \& Trading Co., Ltd. located in Rm1502 haitong Tower,100 Qinhuai Rd Jiangning, Nanjing 211100, Nanjing, Jiangsu, China.

[112] Yangzhou Shenzhou Wind Generator Co. ,Ltd. Xinhe Industrial Park, Xiannv Town, Jiangdu City, Jiangsu Province, China

[113] Aeolos Wind Energy Co., Ltd. No.16, Shandong Road, Qingdao 266000, China.

[114] European Wind Energy Association Wind energy - Volume 2 - the facts - costs \&prices. Available at :

http://www.ewea.org/fileadmin/ewea_documents/documents/publications/WETF/Facts_ Volume_2.pdf. Last accessed on June $21^{\text {st }} 2016$.

[115] A. Ragheb, and M. Ragheb, "Wind turbine gearbox technologies," $1^{\text {st }}$ International Nuclear \& Renewable Energy Conference, 2010, pp. 1 - 8.

[116] J. Seaman, "Rare earth and clean energy: analyzing china upper hand," Institut francais des relations internationales ifri, 2010; pp. 1-34. Available at: https://www.ifri.org/sites/default/files/atoms/files/noteenergieseaman.pdf. Last accessed on June $21^{\text {st }} 2016$.

[117] M.R. David, "Direct Field-oriented control of an induction machine using an adaptive rotor resistance estimator," MSc thesis, The Pennsylvania State University, 2009. 
[118] S. Chiniforoosh, "Generalized dynamic average modeling of line-commutated converter systems in transient simulation programs," $\mathrm{PhD}$ Thesis at University of British Columbia, BC, CANADA, 2012. Available at: https://open.library.ubc.ca/ cIRcle/collections/ubctheses/24/items/1.0072660. Last accessed on June $21^{\text {st }} 2016$.

[119] H. Siegfried, "Grid Integration of wind Energy conversion systems," John Wiley \& Sons Ltd, 1998, ISBN 0-471-97143-X.

[120]Z. Xuesong, Li Ji , and Ma Youjie, "Review on Wind Speed Model Research in Wind Power Systems Dynamic Analysis ", IEEE conference on Sustainable Power Generation and Supply, pp. 1-5, April 2009.

[121] C. Nichita, D. Luca, B. Dakyo, and E. Ceanga, " Large band simulation of the wind speed for real time wind turbine simulators", IEEE Trans. on Energy Conversion, vol. 17, No. 4, Dec. 2002, pp $523-529$.

[122] U.S. Military Specification MIL-F-8785C, 5 November 1980.

[123] I. Florin, A.D. Hansen, P. Sorensen, and F. Blaabjerg, "Wind turbine blockset in Matlab/Simulink," Ris $\phi$, Alborg University. March 2004, pp. 25-28. Available at: http://www.academia.edu/6071102/Wind_Turbine_Blockset_in_Matlab_Simulink. Last accessed on June $21^{\text {st }} 2016$.

[124] M.G. Simoes, and F.A. Farret. Renewable Energy Systems: Design and Analysis with Induction Generators. $1^{\text {st }}$ ed. USA, CRC Press LLC, 2004.

[125] R.C. Bansal, "Three-phase self-excited induction generators: an overview," IEEE Transactions on Energy Conversion, vol. 20, no. 2, pp. 292-299, June 2005.

[126] S. S. Chiniforoosh, H. Atighechi, A. Davoodi, J. Jatskevich, A. Yazdani, S. Filizadeh,M. Saeedifard, J. A. Martinez, V. Sood, K. Strunz, J.Mahseredjian, and V. Dinavahi, "Dynamic average modeling of front-end diode rectifier loads considering discontinuous conduction mode and unbalanced operation," IEEE Trans. Power Del., vol. 27, no. 1, pp. 421-429, Jan. 2012.

[127] D.S. Martin, J. L. R. Amenedo, and S. Arnalte, "Direct power control applied to doubly fed induction generator under unbalanced grid voltage conditions," IEEE Transactions on Power Electronics, Vol. 23, No. 5, pp. 2328-2336, Sep. 2008 
[128] M. N. Zaggout , P. J. TAVNER, and L. RAN, "Wind turbine doubly fed induction generator fault detection based on control loop signals," EWEA Europe's Premier Wind Energy Event, COPENHAGEN, April 16-19, 2012.

[129] H.L.Willis, and W.G.Scott. Distribution Power Generation Planning and Evaluation. $1^{\text {st }}$ ed. USA, CRC Print, 2000.

[130] N. Mohan, T.M. Undeland and W.P. Robbins, Power Electronics: Converters, Applications, and Design, $3^{\text {rd }}$ ed., John Wiley \& Sons, Inc., 2003.

[131] C. Cedric, S. Adnan, A.D. Ahmed, and S.M. Muyeen, "Modeling analysis of battery performance for renewable energy application," $15^{\text {th }}$ European Conf. on Power electronics and applications EPE, Sept. 2-6 2013, pp 1-10.

[132] N. Moubayed, J.Kouta, A. El-Ali H. Dernayka, and R. Outbib, "Parameter identification of the lead-acid battery model," in Proc. Photovoltaic Spec.Conf., May 2008, pp. 1-6.

[133] N. Pogaku, M. Prodanovic, and T C. Green, "Modeling, analysis and testing of autonomous operation of an inverter-based microgrid," IEEE Trans. Power Electron., vol. 22, no. 2, pp. 613-625, Mar. 2007.

[134] R. Majumder, B. Chaudhuri, A. Ghosh, R. Majumder, G. Ledwich, and F. Zare, "Improvement of stability and load sharing in an autonomous microgrid using supplementary droop control loop," IEEE Trans. Power Delivery, vol. 25, no. 2, pp. 796808, May 2010

[135] M. B. Delghavi and A. Yazdani, “A control strategy for islanded operation of a distributed resource (DR) unit," in Proc. IEEE Power Energy Soc. Gen. Meeting, Jul. 2009, pp. 1-8.

[136] F. Katiraei and M. R. Iravani, "Power management strategies for a microgrid with multiple distributed generation units," IEEE Trans. Power Syst., vol. 21, no. 4, pp. 1821-1831, Nov. 2006.

[137] G. Diaz, C. Gonzalez-Moran, J. Gomez-Aleixandre, and A. Diez, "Composite loads in stand-alone inverter-based microgrids-Modeling procedure and effects on load margin," IEEE Trans. Power Syst., vol. 25, no. 2, pp. 894-905, May 2010.

[138] A. Haddadi, A. Yazdani, G. Joos, and B. Boulet, "A generic load model for simulation studies of microgrids," presented at the IEEE Power Eng. Soc. Gen. Meeting, Vancouver, BC, Canada, Jul. 2013. 
[139] A. Haddadi," Modeling, control, and stability analysis of an islanded microgrid", $\mathrm{PhD}$. Dissertation, Dept. ECE, Univ. McGill, CANADA, 2015. Available: http://digitool.library.mcgill.ca/R/?func=dbin-jump-full\&object_id=135516\&local_base $=$ GEN01-MCG02. Last accessed on June $21^{\text {st }} 2016$.

[140] P.T. Cheng, C.-A. Chen, T.-L. Lee, and S.-Y. Kuo, "A cooperative imbalance compensation method for distributed-generation interface converters," IEEE Trans. Ind. Appl., vol. 45, no. 2, pp. 805-815, Mar./Apr.2009.

[141] T.Wizelius. Developing wind power projects: Theory and Practice. 1st ed. UK and USA, Earthscan, 2007.

[142] E. D. Basset and F. M. Potter, "Capacitive excitation for induction generators," Trans. Amer. Inst. Elect. Eng., vol. 54, no. 5, pp. 540-545, May 1935.

[143] A. Kishore, and G. S. kumar, "A Generalized State-Space Modeling of Three Phase SelfExcited Induction Generator for Dynamic Characteristics and Analysis" IEEE, ICIEA, pp $1-6,2006$.

[144] J. D. Aplevich, The Essentials of Linear State-Space Systems, New York: Wiley, 1999.

[145] K. Zhou, G. Salomon, and E.WU. "Balanced Realization and Model reduction for Unstable Systems," Int. Journal of Robust and Nonlinear Control. Vol. 9, pp.183-198. 1999.

[146] Z. Gao, "Scaling and Bandwidth-Parameterization Based Controller Tuning," in Proc. of the American Control Conference, 2003, pp. 4989-4996.

[147] S. M. Raza Kazmi, H. Goto, G. Hai-Jiao, and O. Ichinokura, "Review and critical analysis of the research papers published till date on maximum power point tracking in wind energy conversion system," in Proc. IEEE Energy Convers. Cong. Exposition (ECCE), Sep. 2010, pp. 4075-4082.

[148] D. Kumar, and K. Chatterjee, "A review of conventional and advanced MPPT algorithms for wind energy systems," Renewable and Sustainable Energy Reviews (ELESIVER), vol. 55, March 2016, Pages 957-970.

[149] J. D. M. De Kooning, B. Meersman, T.L. Vandoorn, and L. Vandevelde," Evaluation of the Maximum Power Point Tracking performance in small wind turbines," Power and Energy Society General Meeting, 2012 IEEE , pp.1,8, 22-26, July 2012. 
[150] K. Kyung-Hwan, P. Nam-Joo, and H. Dong-Seok, "Advanced synchronous reference frame controller for three-phase UPS powering unbalanced and nonlinear loads," in Proc. Power Electron. Specialists Conf., 2005, pp. 1699-1704.

[151] I. Vechiu, O. Curea, and H. Camblong, "Transient operation of a four-leg inverter for autonomous applications with unbalanced load," IEEE Trans. Power Electron., vol. 25, no. 2, pp. 399-407, Feb. 2010.

[152] H. L. Wills. Spatial Electric Load Forecasting. $2^{\text {nd }}$ ed. Marcel Dekker, Inc. New York, USA, 2002.

[153] C. Tang, M. Pathmanathan, W.L. Song, and N. Ertugrul, "Effects of Inertia on dynamic performance of wind Turbines," 2008 Australian Universities Power Engineering Conference (AUPEC'08), pp.1-6, Dec. 2008.

[154] D. N. Zmood, and D. G. Holmes, "Stationary Frame Current Regulation of PWM Inverters with Zero Steady-State Error" IEEE Trans. on Power Electr., vol. 18, no. 3, May 2003, pp. $814-822$.

[155] Z. Alnasir, and M. Kazerani, "A small-scale standalone wind energy conversion system featuring SCIG, CSI and a novel storage integration scheme," Journal of Renewable Energy, ELSEVIER, vol. 89, pp. 360-370, 2016.

[156] Z. Alnasir, and M. Kazerani, "A dump load-less standalone wind energy conversion system supplying a generic load," accepted by the IEEE Electrical Power \& Energy Conference EPEC 2016, October 12-14, 2016, Ottawa, Canada.

[157] S. Anand, S. K. Gundlapalli, and B. G. Fernandes, "Transformer-less grid feeding current source inverter for solar photovoltaic system," IEEE Trans. Ind. Electron., vol. 61, no. 10, pp. 5334-5344, Oct. 2014. 